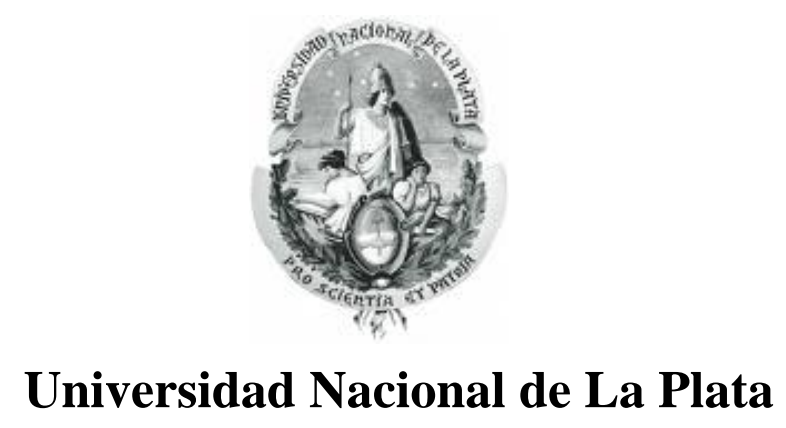

Facultad de Ciencias Exactas

Departamento de Física

Tesis

Para optar al título de

Doctor en Ciencias Exactas: Orientación Física

\title{
Estudio de la microestructura y propiedades magnéticas de aleaciones FeMnCu
}

Tesista: Lic. Martín Mizrahi 
Mizrahi, Martín Daniel

Estudio de la microestructura y propiedades magnéticas de aleaciones FeMnCu. - 1a ed. - La Plata : Universidad Nacional de La Plata, 2011.

CD-ROM.

ISBN 978-950-34-0782-0

1. Aleaciones. 2. Tesis. I. Título CDD 669

Fecha de catalogación: 08/11/2011 


\title{
Tesis Doctoral
}

\author{
Realizada en el \\ Departamento de Física
}

\author{
presentada en la \\ Facultad de Ciencias Exactas \\ Universidad Nacional de La Plata
}

para obtener el Título de
Doctor de la Facultad de Ciencias Exactas

por

Martín Mizrahi

Tema: Estudio de la microestructura y propiedades magnéticas de aleaciones $\mathrm{FeMnCu}$.

Dirección: Prof. Dra. Judith Desimoni.

Co-dirección: Prof. Dra. Alejandra Fabiana Cabrera.

Sostenida el 30 de marzo de 2010, frente al jurado de Tesis

Prof. Dra. Bibiana Arcondo

Prof. Dr. Francisco Sánchez

Prof. Dr. Gustavo Echeverría 


\section{Contenidos}

Capítulo 1. Introducción ............................................................................................................... 1

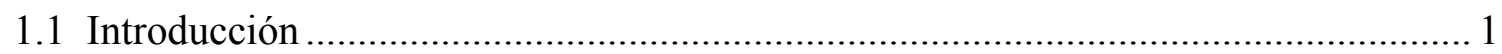

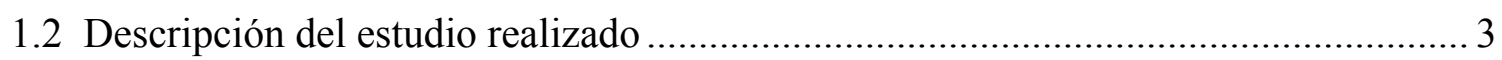

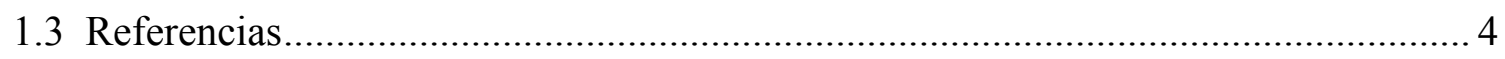

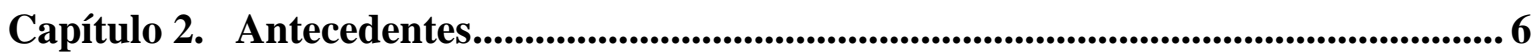

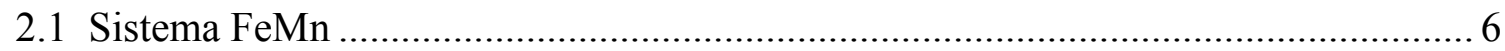

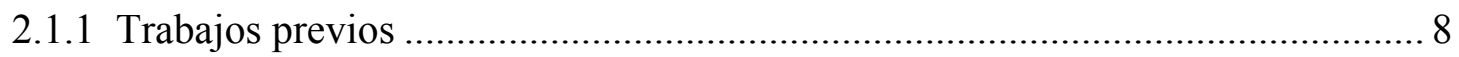

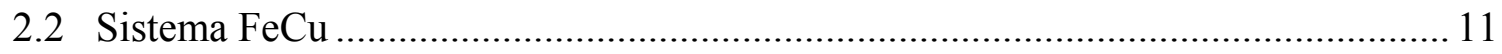

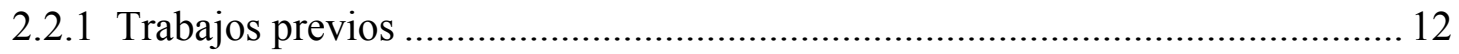

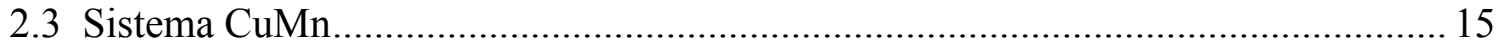

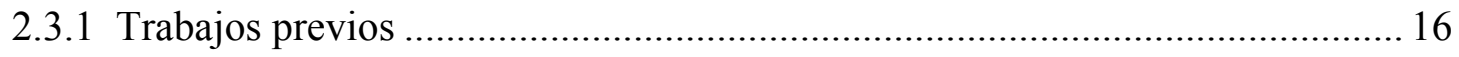

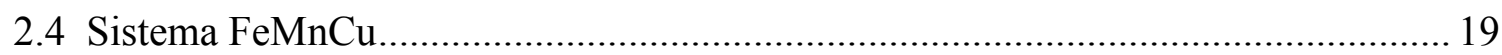

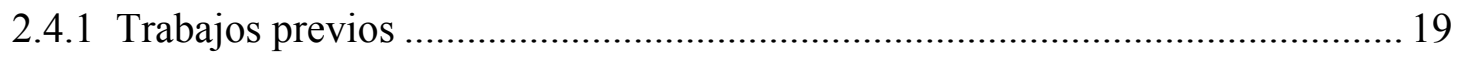

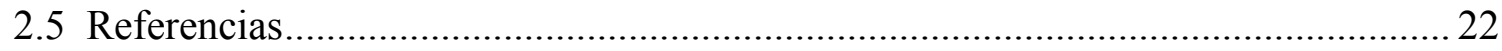

Capítulo 3. Algunas propiedades magnéticas de sistemas nanoestructurados ........... 25

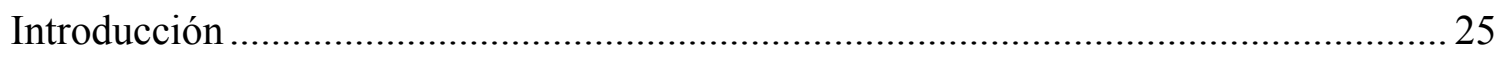

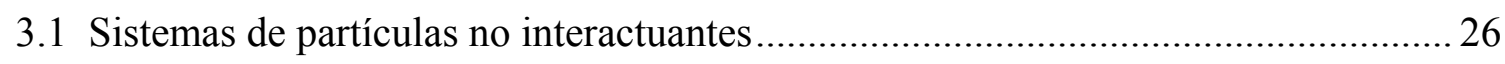

3.1.1 Tiempos de relajación y bloqueo del sistema ................................................. 26 
3.2 Conjuntos de partículas, fórmula de Langevin

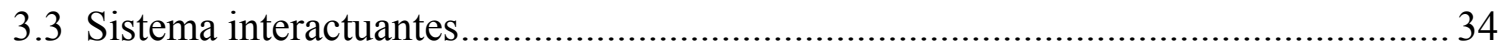

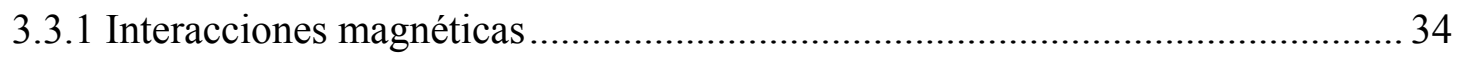

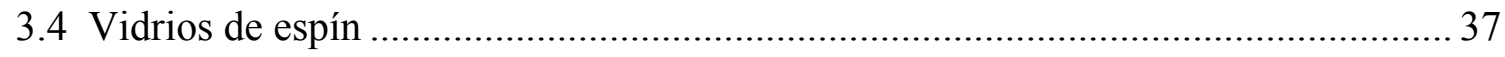

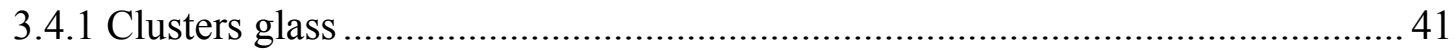

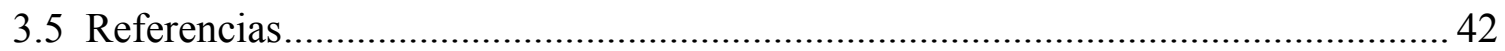

Capítulo 4. Técnicas experimentales y análisis de datos.............................................. 44

4.1 Técnica de aleado mecánico y preparación de las muestras ................................... 44

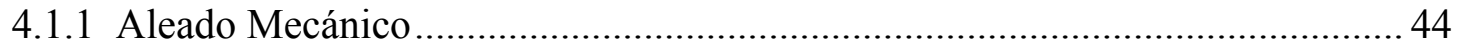

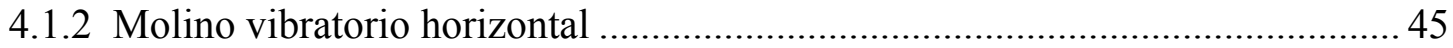

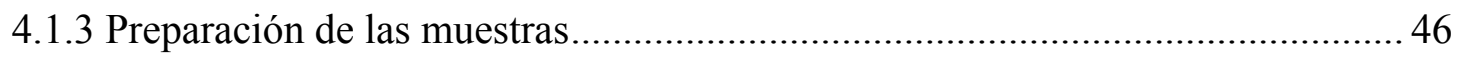

4.1.4 Fraccionamiento y montaje de muestras ..................................................... 47

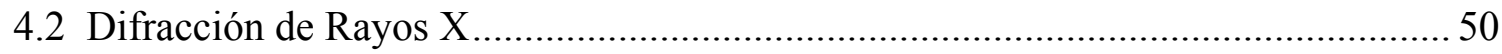

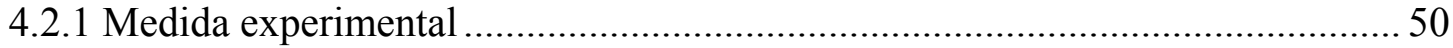

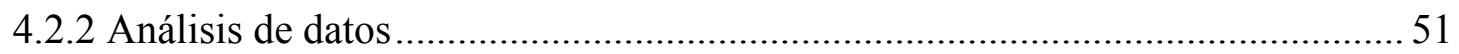

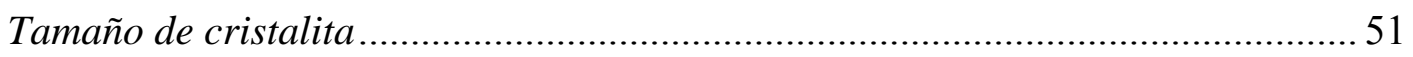

4.3 Espectroscopia de Absorción de Rayos X .......................................................... 53

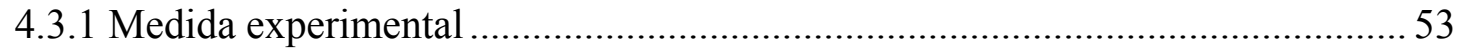

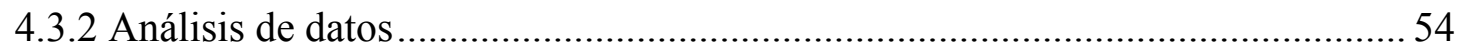

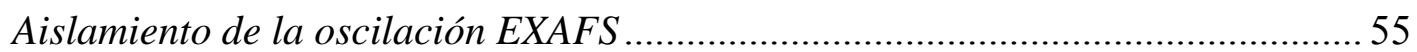

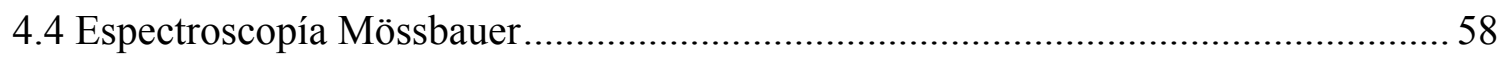

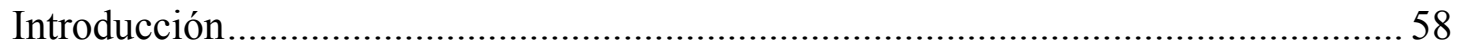

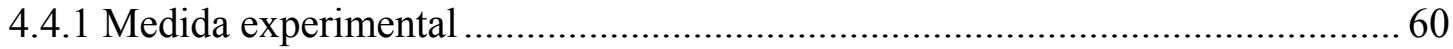

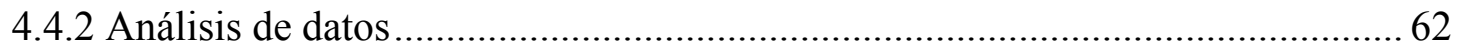

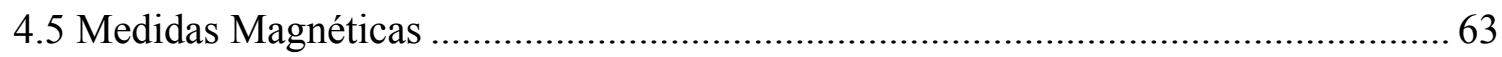


4.5.1 Medida experimental

4.5.2 Análisis de datos

4.6 Referencias.

Capítulo 5. Resultados y discusión.

5.1 Materiales precursores

5.2 Cinéticas de Molienda

5.2.2 Espectroscopía Mössbauer

5.3 Evolución con el contenido de $\mathrm{Cu}$ de los parámetros estructurales, hiperfinos y magnéticos

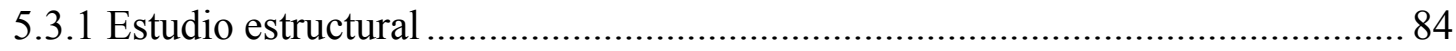

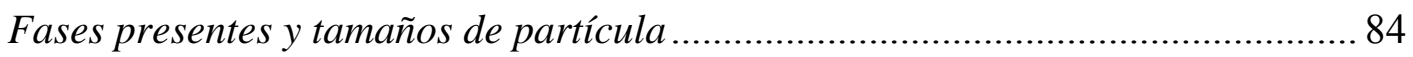

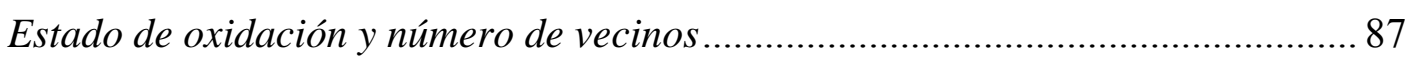

Modelo estadístico de distribución atómica ............................................................. 93

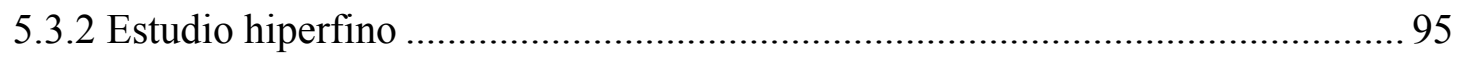

Espectroscopía Mössbauer a temperatura ambiente ........................................... 95

Espectroscopía Mössbauer a bajas temperaturas............................................. 102

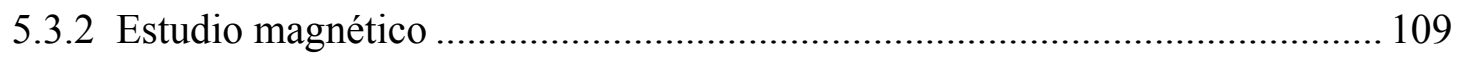

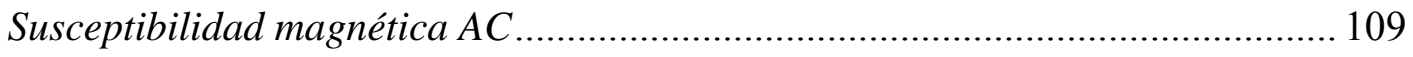

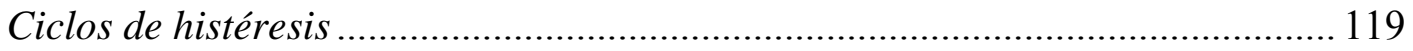

Medidas de magnetización con y sin campo (FC-ZFC) ...................................... 142

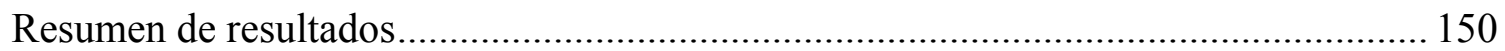

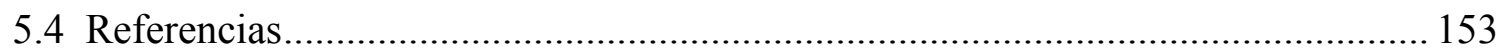

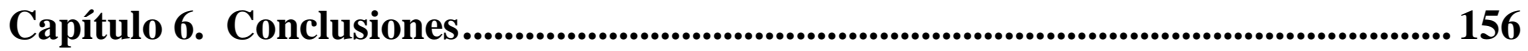

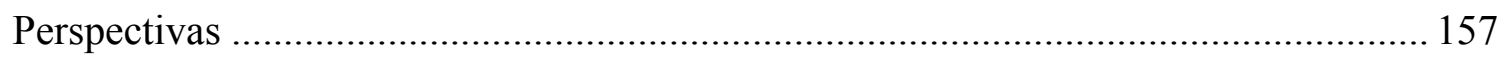

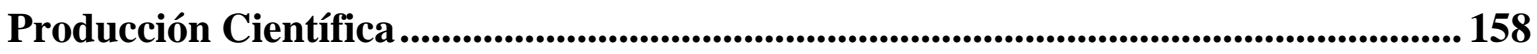

Agradecimientos ................................................................................................................... 159 


\section{Índice de Figuras}

Figura 2.1: Diagrama de fases del sistema FeMn en función del porcentaje de Mn.

Figura 2.2: Diagrama de fases del sistema $\mathrm{FeCu}$ función de la fracción de $\mathrm{Fe}$.

Figura 2.3: Diagrama de fases del sistema CuMn en función del porcentaje de Mn.

Figura 3.1: a) Esquema de la partícula considerada con los ejes de magnetización y dirección del campo magnético aplicado. b) Dependencia angular de la barrera de energía sin campo aplicado (línea sólida) y en presencia de un campo magnético externo (línea punteada).

Figura 3.2: Variación del radio crítico y del tiempo de relajación en función de la temperatura para una barrera de energía fija.

Figura 3.3: Comportamiento de la función de Langevin en función de $\mathrm{H} / \mathrm{T}$ para distintos valores del parámetro $\alpha$

Figura 3.4: Curvas de magnetización ZFC y FC para el vidrio de espín canónico CuMn con los porcentajes de $\mathrm{Mn}$ indicados un la figura.

Figura 4.1: Fotografía del molino vibratorio horizontal, similar al empleado para la preparación de las muestras de esta tesis (izq). Cilindros y esferas usados en el proceso de molienda (der).

Figura 4.2: Metales empleados para la preparación de las muestras: Hierro (izq.), Manganeso (centro) y Cobre (der).

Figura 4.3: Esquema del soporte empleado para realizar las medidas de difracción de rayos X.... 48

Figura 4.4: Esquema del montaje de una muestra para ser medida por espectroscopía Mössbauer.

Figura 4.5: Esquema del montaje de la muestra para la realización de las medidas magnéticas.... 49

Figura 4.6: Esquema del montaje de la muestra para realizar las medidas de absorción de rayos X.

Figura 4.7: Difractómetro Philips $X$ 'Pert Pro utilizado para realizar las medidas de difracción de rayos $\mathrm{X}$ (izq). Fotografía del goniómetro del difractómetro junto con un esquema de la geometría de trabajo del instrumento (der).

Figura 4.8: Fotografía del anillo acelerador de electrones ubicado en el Laboratorio Nacional de Luz Sincrotrón. 
Figura 4.9: Esquema simplificado del arreglo empleado para realizar las medidas de absorción de rayos $\mathrm{X}$ para el caso de la detección por fluorescencia.

Figura 4.10: Etapas del tratamiento de la señal EXAFS. a) restado de línea de base, b) normalizado, c) determinación de energía de borde y d) remoción de la absorción atómica. ........ 56

Figura 4.11: Transformada de Fourier del espectro de absorción de rayos $\mathrm{X}$ para el $\mathrm{Cu}$ medido en el borde $\mathrm{K}$.

Figura 4.12: Esquema simplificado del arreglo experimental empleado para realizar las medidas de espestroscopía Mössbauer en geometría de transmisión.

Figura 4.13: Diagrama de la disposición experimental de la electrónica de comando de un espectrómetro Mössbauer.....

Figura 4.14: Fotografías de los equipos de magnetismo: Susceptómetro (izq.), PPMS (centro) y MPMS (der.).

Figura 4.15: Corte transversal del cilindro criogénico del dispositivo PPMS (izq). Detalle de la región contenedora de la muestra donde se encuentran las bobinas de excitación y medición (centro). Varilla para soporte del portamuestra (der).

Figura 5.1: Difractograma de Fe metálico molido durante 15 min. Las marcas al pie $(\mid)$ de la figura indican la posición de las líneas de la fase bcc. 70

Figura 5.2: Difractograma de Mn metálico molido durante 15 min. Las marcas al pie ( $\mid$ ) de la figura indican la posición de las líneas de la fase $\alpha-\mathrm{Mn}$. 70

Figura 5.3: Difractograma de Cu metálico molido durante 15 min. Las marcas al pie ( | ) de la figura indican la posición de las líneas esperada según la bibliografía. 71

Figura 5.4: Ciclo de histéresis del Fe molido 15 h. La línea sólida es el resultado del ajuste. 71

Figura 5.5: Ciclo de histéresis del Mn molido $15 \mathrm{~h}$. La línea a trozos representa la contribución ferromagnética mientras la línea punteada representa la contribución paramagnética. 72

Figura 5.6: Ciclo de histéresis del Cu sin moler. La línea sólida es el resultado del ajuste. 72

Figura 5.7: Difractogramas de las muestras con $\mathrm{x}=0.15 \mathrm{y} \mathrm{x}=0.30$ para los diferentes tiempos de molienda junto con la posición de las líneas simuladas para las fases $f c c-\mathrm{Fe}_{79} \mathrm{Mn}_{21}(\mid)$ y bcc$\mathrm{Fe}_{90} \mathrm{Mn}_{10}(\Delta)$. En ambos difractogramas se indican los índices de Miller de ambas fases.

Figura 5.8: Variación del tamaño medio de cristalita con el tiempo de molienda para la muestra con $\mathrm{x}=0.15$ (cuadrados sólidos) y $\mathrm{x}=0.30$ (círculos abiertos).

Figura 5.9: Variación del parámetro de red con el tiempo de molienda para la muestra con $\mathrm{x}=0.15$ (cuadrados sólidos) y $\mathrm{x}=0.30$ (círculos abiertos).

Figura 5.10: Espectros Mössbauer adquiridos en el rango mayor de velocidades para la muestra con $\mathrm{x}=0.15$ (izq.) y $\mathrm{x}=0.30$ (der.) para los distintos tiempos de molienda. Las líneas sólidas corresponden al resultado de los ajustes de los espectros. 79 
Figura 5.11: Espectros Mössbauer adquiridos en el rango menor de velocidades de las muestras con $\mathrm{x}=0.15$ (izq.) y $\mathrm{x}=0.30$ (der.) para los distintos tiempos de molienda. Las líneas sólidas corresponden al resultado del ajuste de los espectros.

Figura 5.12: Distribución de probabilidades de la interacción de alto campo de las muestras con $x$ $=0.15$.

Figura 5.13: Distribución de probabilidades de la interacción de bajo campo.

Figura 5.14: Campo magnético hiperfino (izq.) y corrimiento isomérico (der.) en función del tiempo de molienda para la componente de campo bajo. En ambas figuras los cuadrados sólidos y los círculos abiertos corresponden a $\mathrm{x}=0.15 \mathrm{y} \mathrm{x}=0.30$, respectivamente. 83

Figura 5.15: Difractogramas de las muestras para las concentraciones de $\mathrm{Cu}$ junto con la posición de las líneas simuladas para las fases $f c c-\mathrm{Fe}_{79} \mathrm{Mn}_{21}(\mid)$ y $b c c-\mathrm{Fe}_{90} \mathrm{Mn}_{10}(\Delta)$..

Figura 5.16: Variación del parámetro de red con el contenido de $\mathrm{Cu}$. 86

Figura 5.17: Imagen de microscopía electrónica de transmisión correspondiente a la muestra con $\mathrm{x}$ $=0.20$ (izq.). Distribución de tamaños de partícula estimados a partir de la imagen TEM (der.), la línea sólida representa el ajuste con una distribución log-normal de los datos experimentales. ..... 87

Figura 5.18: Región XANES del espectro de absorción medido en el borde $K$ del Fe.................... 88

Figura 5.19: Región XANES del espectro de absorción medido en el borde $K$ del $\mathrm{Cu}$. 88

Figura 5.20: Comparación de la región XANES correspondientes a espectros del Fe metálico y óxidos de Fe.

Figura 5.21: Derivada de los espectros de absorción en la región XANES (línea sólida) con las correspondientes al $\mathrm{Fe}$ metálico (línea a trozos). La línea vertical indica la energía del borde $K$ del $\mathrm{Fe}\left(\mathrm{E}_{\mathrm{b}}=7112 \mathrm{eV}\right)$

Figura 5.22: Comparación de la derivada de los espectros de absorción en la región XANES (línea sólida) con las correspondientes al $\mathrm{Cu}$ metálico (línea a trozos). La línea vertical indica la energía del borde $K$ del $\mathrm{Cu}\left(\mathrm{E}_{\mathrm{b}}=8979 \mathrm{eV}\right)$.

Figura 5.23: Espectros de absorción de rayos $X$ para distintas concentraciones.

Figura 5.24: Transformada de Fourier de la región EXAFS de los espectros medidos en el borde $K$ del $\mathrm{Cu}$ junto con el ajuste (línea roja). El recuadro es una ampliación del pico correspondiente a la primera esfera de coordinación.

Figura 5.25: Variación de la distancia a primeros vecinos para las distintas concentraciones estudiadas obtenidas de los ajustes de los espectros EXAFS, junto con un ajuste lineal de los datos (línea sólida).

Figura 5.26: Valores de $N_{\mathrm{Cu}}$ obtenidos de los ajustes EXAFS (cuadrados abiertos) y según la predicción estadística $\left(N^{*}\right.$ Cu (círculos sólidos), junto con los ajustes lineales correspondientes (línea sólida) y (línea punteada), respectivamente. 
Figura 5.27: Probabilidad de encontrar $n_{\mathrm{Cu}}$ átomos de $\mathrm{Cu}$ primeros vecinos según lo predicho por la Ec.5.1 para las distintas concentraciones estudiadas. La línea continua representa el ajuste lognormal de las probabilidades calculadas.

Figura 5.28: Espectros Mössbauer en el rango mayor de velocidades. Las líneas sólidas corresponden al resultado de los ajustes.

Figura 5.29: Espectros Mössbauer medidos en el rango de velocidades menor. La línea vertical indica el valor del corrimiento isomérico para la muestra $\mathrm{x}=0.00$.

Figura 5.30: Espectros Mössbauer medidos en el rango menor de velocidades junto con las correspondientes DCMH. La línea continua en los espectros representa el ajuste.

Figura 5.31: Parámetro de red determinado a partir de las posiciones angulares des las líneas (111) (cuadrados abiertos) y (200) (círculos sólidos).

Figura 5.32: Parámetros obtenidos de los ajustes en función del contenido de $\mathrm{Cu}$. Campo hiperfino medio (izq.) y corrimiento isomérico medio (der.). La línea continua representa un ajuste lineal.

Figura 5.33: Espectros Mössbauer medidos a varias temperaturas para la muestra con $\mathrm{x}=0.00$ junto con las correspondientes DCMH. La línea continua en los espectros representa el ajuste... 103

Figura 5.34: Espectros Mössbauer medidos a varias temperaturas para la muestra con $\mathrm{x}=0.10$ junto con las correspondientes DCMH. La línea continua en los espectros representa el ajuste... 103

Figura 5.35: Espectros Mössbauer medidos a varias temperaturas para la muestra con $\mathrm{x}=0.15$ junto con las correspondientes DCMH. La línea continua en los espectros representa el ajuste... 104

Figura 5.36: Espectros Mössbauer medidos a varias temperaturas para la muestra con $\mathrm{x}=0.20$ junto con las correspondientes DCMH. La línea continua en los espectros representa el ajuste... 104

Figura 5.37: Espectros Mössbauer medidos a varias temperaturas para la muestra con $\mathrm{x}=0.25$ junto con las correspondientes DCMH. La línea continua en los espectros representa el ajuste... 105

Figura 5.38: Espectros Mössbauer medidos a varias temperaturas para la muestra con $\mathrm{x}=0.30$ junto con las correspondientes DCMH. La línea continua en los espectros representa el ajuste... 105

Figura 5.39: Evolución con la temperatura del campo hiperfino medio. Las líneas punteadas son una guía para la vista. 107

Figura 5.40: Evolución con la temperatura del corrimiento isomérico medio. Las líneas punteadas son una guía para la vista. 108

Figura 5.41: Susceptibilidad magnética en función de la temperatura. 110

Figura 5.42: Variación de la temperatura del máximo de la susceptibilidad en función del contenido de $\mathrm{Cu}$ (abscisa inferior) y del número más probable de átomos de $\mathrm{Cu}$ primeros vecinos (abscisa superior). 111

Figura 5.43: Variación de la susceptibilidad con la temperatura, para distintas frecuencias del campo de excitación. A la derecha se muestra la región del máximo ampliada. 112 
Figura 5.44: Variación del $\ln (\tau)$ en función de $1 /\left(\mathrm{T}_{\max }-\mathrm{T}_{0}\right)$ para las muestras $\mathrm{x}=0.20,0.25$ y 0.30 . La línea sólida es el ajuste lineal obtenido.

Figura 5.45: Variación del tiempo característico de medida en función de la temperatura del máximo de la susceptibilidad (izq.) junto con el ajuste según la Ec. 5.9 (línea sólida). log $(\tau)$ en función de $\log \left[\left(\mathrm{T}_{\max } / \mathrm{T}_{\mathrm{c}}-1\right)\right]$ (der.) junto al ajuste lineal a los datos (línea sólida).

Figura 5.46: Variación de la componente en fase de la susceptibilidad AC en la muestra $\mathrm{x}=0.30$ en presencia de un campo magnético estático $\mathrm{H}_{\mathrm{DC}}$

Figura 5.47: Variación del coeficiente $a_{3}$ de la susceptibilidad no lineal con la temperatura.

Figura 5.48: Ciclos de histéresis a $300 \mathrm{~K}$ (izq.). Región central de los ciclos (der.).

Figura 5.49: Medidas de histéresis entre $300 \mathrm{~K}$ y $5 \mathrm{~K}$ para la muestra con $\mathrm{x}=0.00$ (izq.). Región central de los ciclos (der.).

Figura 5.50: Medidas de histéresis entre $300 \mathrm{~K}$ y $5 \mathrm{~K}$ para la muestra con $\mathrm{x}=0.10$ (izq.). Región central de los ciclos (der.).

Figura 5.51: Medidas de histéresis entre $300 \mathrm{~K}$ y $5 \mathrm{~K}$ para la muestra con $\mathrm{x}=0.15$ (izq.). Región central de los ciclos (der.).

Figura 5.52: Medidas de histéresis entre $300 \mathrm{~K}$ y $5 \mathrm{~K}$ para la muestra con $\mathrm{x}=0.20$ (izq.). Región central de los ciclos (der.).

Figura 5.53: Medidas de histéresis entre $300 \mathrm{~K}$ y $5 \mathrm{~K}$ para la muestra con $\mathrm{x}=0.25$ (izq.). Región central de los ciclos (der.).

Figura 5.54: Medidas de los ciclos de histéresis entre $300 \mathrm{~K}$ y $5 \mathrm{~K}$ para la muestra con $\mathrm{x}=0.30$ (izq.). Región central de los ciclos (der.).

Figura 5.55: Derivadas de la rama izquierda de los ciclos de histéresis para distintas temperaturas.

Figura 5.56: Resultados de los ajustes de los ciclos de histéresis medidos a bajas temperaturas para la muestra con $\mathrm{x}=0.00$. Ajuste propuesto (línea negra) y las distintas contribuciones al mismo: ferromagnética 1 (línea roja), ferromagnéticas 2 (línea verde claro), Langevin (línea verde oscura) y lineal (línea azul).

Figura 5.57: Resultados de los ajustes de los ciclos de histéresis medidos a bajas temperaturas para la muestra con $\mathrm{x}=0.10$. Ajuste propuesto (línea negra) y las distintas contribuciones al mismo: ferromagnética 1 (línea roja), ferromagnéticas 2 (línea verde claro), Langevin (línea verde oscura) y lineal (línea azul).

Figura 5.58: Resultados de los ajustes de los ciclos de histéresis medidos a bajas temperaturas para la muestra con $\mathrm{x}=0.15$. Ajuste propuesto (línea negra) y las distintas contribuciones al mismo: ferromagnética 1 (línea roja), ferromagnéticas 2 (línea verde claro), Langevin (línea verde oscura) y lineal (línea azul).

Figura 5.59: Resultados de los ajustes de los ciclos de histéresis medidos a bajas temperaturas para la muestra con $\mathrm{x}=0.20$. Ajuste propuesto (línea negra) y las distintas contribuciones al mismo: 
ferromagnética 1 (línea roja), ferromagnéticas 2 (línea verde claro), Langevin (línea verde oscura) y lineal (línea azul).

Figura 5.60: Resultados de los ajustes de los ciclos de histéresis medidos a bajas temperaturas para la muestra con $\mathrm{x}=0.25$. Ajuste propuesto (línea negra) y las distintas contribuciones al mismo: ferromagnética 1 (línea roja), ferromagnéticas 2 (línea verde claro), Langevin (línea verde oscura) y lineal (línea azul).

Figura 5.61: Resultados de los ajustes de los ciclos de histéresis medidos a bajas temperaturas para la muestra con $\mathrm{x}=0.30$. Ajuste propuesto (línea negra) y las distintas contribuciones al mismo: ferromagnética 1 (línea roja), ferromagnéticas 2 (línea verde claro), Langevin (línea verde oscura) y lineal (línea azul).

Figura 5.62: Evolución de la magnetización de saturación para las diferentes concentraciones estudiadas a temperatura ambiente.

Figura 5.63: Ciclo de histéresis correspondiente a la muestra $\mathrm{Fe}_{90} \mathrm{Mn}_{10}$ medido a temperatura ambiente, junto con el ajuste propuesto (para mayor claridad se muestra sólo una rama del ciclo). Recuadro: ampliación de la región central del ciclo.

Figura 5.64: Magnetización de saturación de la componente ferromagnética 1 obtenida de los ajustes de los ciclos entre $300 \mathrm{~K}$ y $5 \mathrm{~K}$. 132

Figura 5.65: Magnetización de saturación de la componente ferromagnética 2 obtenida de los ajustes de los ciclos entre $300 \mathrm{~K}$ y $5 \mathrm{~K}$. 132

Figura 5.66: Campo coercitivo de la componente ferromagnética 1 obtenida de los ajustes de los ciclos entre $300 \mathrm{~K}$ y $5 \mathrm{~K}$. 134

Figura 5.67: Campo coercitivo de la componente ferromagnética 2 obtenida de los ajustes de los ciclos entre $300 \mathrm{~K}$ y $5 \mathrm{~K}$. 134

Figura 5.68: Resultados para la componente lineal obtenida de los ajustes de los ciclos entre $300 \mathrm{~K}$ y $5 \mathrm{~K}$

Figura 5.69: Ciclos de histéresis para la muestra $x=0.00$ enfriada en un campo magnético de 20 kOe.

Figura 5.70: Ciclos de histéresis para la muestra $x=0.20$ enfriada en un campo magnético de 20 kOe.

Figura 5.71: Ciclos de histéresis para la muestra $x=0.30$ enfriada en un campo magnético de 20 kOe.

Figura 5.72: Comportamiento del campo de exchange bias (izq) y el campo coercitivo medio (der.) en función de la temperatura para las muestras $\mathrm{x}=0.00, \mathrm{x}=0.20 \mathrm{y} \mathrm{x}=0.30$. 138

Figura 5.73: Arriba: Ciclo de histéresis para la muestra $\mathrm{x}=0.30$ medido a $5 \mathrm{~K}$ para distintos campos de enfriado $\left(H_{F C}\right)$. Abajo: ampliación de la región central del ciclo. 139

Figura 5.74: Campo de exchange bias en función del campo magnético de enfriado. 140 
Figura 5.75: Campo de exchange bias medida a $5 \mathrm{~K}$ después de ser enfriado en un campo de 20 kOe desde la temperatura $T_{F C}$

Figura 5.76: Magnetización en función de la temperatura en FC y ZFC para campos de enfriado de 20 Oe (izq.) y 100 Oe (der.). 142

Figura 5.77: Magnetización FC (círculos huecos) y ZFC (cuadrados sólidos) en función de la temperatura en para la muestra $\mathrm{x}=0.30$. 144

Figura 5.78: Variación de $T_{\max }$ y $T_{Z F C}$ en función del campo magnético. Las líneas sólidas representan el ajuste de los datos experimentales. 146

Figura 5.79: Dependencia del logaritmo de la frecuencia de medida con la inversa de la temperatura junto con el comportamiento según la Ley de Arrhenius (línea punteada) y el ajuste empleando la Ley de Vogel-Fulcher (línea sólida).

Figura 5.80: Variación del campo coercitivo obtenido con la ecuación 5.14 (cuadrados negros), junto con los resultados provenientes del ajuste de los ciclos de histéresis (círculos rojos). 148 


\section{Índice de Tablas}

Tabla 3.1: Tiempos característicos de medida de diversas técnicas experimentales.

Tabla 3.2: Volúmenes críticos determinados para las diversas técnicas experimentales.

Tabla 5.1: Nomenclatura usada para identificar las muestras estudiadas en función del tiempo de molienda.

Tabla 5.2: Tamaño de cristalita $\left(S_{c}\right)$ y parámetro de red de la fase $f_{c c}\left(a_{f c c}\right)$, para las distintas muestras y tiempos de molienda.

Tabla 5.3: Resultados de los ajustes para el campo magnético hiperfino medio $\left(\mathrm{B}_{\mathrm{hf}}\right)$, momento de $2^{\text {do }}$ orden $(\sigma)$, corrimiento isomérico medio $(\delta)$ y fracción relativa al total del espectro para la interacción de alto y bajo campo (f) en las muestras con $\mathrm{x}=0.15$.

Tabla 5.4: Resultados de los ajustes para el campo magnético hiperfino medio $\left(\mathrm{B}_{\mathrm{hf}}\right)$, momento de $2^{\text {do }}$ orden $(\sigma)$, corrimiento isomérico medio $(\delta)$ y fracción relativa al total del espectro para la interacción de alto y bajo campo (f) en las muestras con $\mathrm{x}=0.30$.

Tabla 5.5: Evolución del tamaño de cristalita $\left(S_{c}\right)$, del parámetro de red de la fase $f c c\left(a_{f c c}\right)$ en función del contenido de $\mathrm{Cu}$.

Tabla 5.6: Distancia a primeros vecinos (D), número de átomos de $\mathrm{Cu}$ primeros vecinos, determinado por EXAFS $\left(\mathrm{N}_{\mathrm{Cu}}\right)$ y número medio de átomos de $\mathrm{Cu}$ primeros vecinos estadístico $\left(\mathrm{N}^{*} \mathrm{Cu}\right)$ en función del contenido de $\mathrm{Cu}$.

Tabla 5.7: Resultados del ajuste para el campo magnético hiperfino medio $\left(\mathrm{B}_{\mathrm{hf}}\right)$, momento de $2^{\text {do }}$ orden $(\sigma)$, corrimiento isomérico medio $(\delta)$ y fracción relativa (f) al total del espectro para la interacción de alto campo

Tabla 5.8: Resultados de los ajustes. Campo hiperfino medio $\left(B_{h f}\right)$, momento de $2^{\text {do }}$ orden $(\sigma)$, corrimiento isomérico medio $(\delta)$ y fracción relativa al total del espectro (f) para la interacción de bajo campo.

Tabla 5.9: Resultados de los ajustes para las distintas temperaturas y concentraciones. 106

Tabla 5.10: Temperatura del máximo de las curvas de susceptibilidad. 
Tabla 5.11: Valores de la temperatura del máximo de $\chi^{\prime}\left(T_{\max }\right)$, de la sensibilidad a la frecuencia $(p)$, del parámetro de Vogel-Fulcher $\left(T_{0}\right)$ y los parámetros de la ley de exponentes críticos $\left(\tau_{0}\right.$ y $\left.z v\right)$.

Tabla 5.12: Resultados de los ajustes de los ciclos de histéresis medidos entre $300 \mathrm{~K}$ y 5 K..... 128

Tabla 5.13: Cociente entre la magnetización de saturación $M_{S 1}$ y la correspondiente a la muestra $\mathrm{Fe}_{90} \mathrm{Mn}_{10}$ junto con la fracción (f) de la fase $\alpha-\mathrm{Fe}(\mathrm{MnCu})$ obtenida por EM.

Tabla 5.14: Temperaturas de irreversibilidad $\left(T_{i}\right)$ y del máximo de la curva ZFC ( $\left.T_{Z F C}\right)$ para las distintas concentraciones y campos magnéticos de enfriado.

Tabla 5.15: Resultados del ajuste de la temperatura del máximo en función del campo magnético aplicado. 


\section{Capítulo 1. Introducción}

\subsection{Introducción}

Las aleaciones nanoestructuradas basadas en hierro son un tópico de permanente interés producto de la gran variedad de nuevas propiedades que presentan y de sus potenciales aplicaciones tecnológicas. ${ }^{1-3}$ Durante la última década, se ha puesto énfasis en el estudio de las propiedades físicas de estos sistemas al ser llevados a la escala nanométrica, ya que en estas condiciones la relación superficie/volumen de las partículas ${ }^{4}$ puede ser lo suficientemente grande para que aparezcan nuevos fenómenos. En este contexto, nuevos modelos han surgido donde se proponen explicaciones para la nueva física observada.,

Por otra parte, es sabido que el método de aleado mecánico permite obtener fases fuera del equilibrio con un gran desorden estructural y tamaños de cristalita del orden de la decena de nanometros. ${ }^{7}$ En sistemas compuestos por átomos magnéticos y no magnéticos, este desorden atómico y la reducción de las longitudes características, dan lugar a la aparición de frustración en las interacciones magnéticas y, por consiguiente, conducen a que el sistema presente diferentes propiedades magnéticas como superparamagnetismo o comportamiento tipo vidrio de espín. ${ }^{8,9}$

En este marco, el sistema FeMnCu puede ser considerado un buen candidato para presentar una diversidad de propiedades interesantes heredadas de las aleaciones binarias de los elementos que la conforman. El sistema $\mathrm{FeCu}$ ha sido estudiado previamente tanto desde el punto de vista de la física básica como de sus potenciales aplicaciones, en particular por los efectos magnetorresistivos observados. ${ }^{10}$ Debido a la entalpía de mezcla positiva entre el Fe y el $\mathrm{Cu}^{11}$ no es sencillo lograr una aleación de los mismos por métodos convencionales y por ello el aleado mecánico es, en este caso, la técnica indicada para obtener la aleación. ${ }^{12}$ El sistema CuMn ha mostrado que para bajos contenidos de Mn tiene un comportamiento de vidrio de espín, ${ }^{13}$ mientras que para grandes concentraciones de Mn exhibe interacciones magnéticas complejas conduciendo al sistema a un estado magnéticamente ordenado de largo alcance. ${ }^{14}$ Por último, el sistema $f c c$ FeMn ha sido empleado para extrapolar las propiedades estructurales y magnéticas de la fase $f c c-$ 
Fe la cual es metaestable a temperatura ambiente. ${ }^{15,16}$ La fase $f c c$-FeMn puede ser obtenida tanto por métodos convencionales ${ }^{17}$ como por aleado mecánico. ${ }^{18}$ Empleando la técnica de aleado mecánico es posible extender la región donde aparece esta fase, ${ }^{19}$ en comparación con la predicha por el correspondiente diagrama de fases de equilibrio. ${ }^{20}$ En lo que respecta al sistema ternario $\mathrm{FeMnCu}$, en varios trabajos se ha estudiado el magnetismo presente en la región de alto contenido de $\mathrm{Cu}^{21,22}$ En estos casos transiciones de alto a bajo espín han sido interpretadas como las causantes del comportamiento del sistema. ${ }^{23}$ En otros trabajos las características magnéticas observadas fueron atribuidas a la segregación de fases binarias. ${ }^{24}$ Sin embargo, no hay antecedentes en la bibliografía del sistema FeMnCu en la región de bajos e intermedios contenidos de $\mathrm{Mn}$ y Cu, salvo los que se presentan en esta Tesis. ${ }^{25-29}$

Considerando entonces que el aleado mecánico es una técnica promisoria para preparar aleaciones nanoestructuraras de $\mathrm{FeMnCu}$, la motivación de esta Tesis es contribuir al entendimiento de las propiedades estructurales, hiperfinas y magnéticas del sistema $\left(\mathrm{Fe}_{79} \mathrm{Mn}_{21}\right)_{1}$ ${ }_{\mathrm{x}} \mathrm{Cu}_{\mathrm{x}}(0.00 \leq \mathrm{x} \leq 0.30)$. La investigación se basa en el estudio del comportamiento del sistema sometido a diferentes tiempos de molienda y del efecto del contenido de $\mathrm{Cu}$. Para tal fin, las muestras fueron caracterizadas empleando Difracción de Rayos-X (DRX), Espectroscopia de Absorción de Rayos-X (XAS), Espectroscopia Mössbauer (EM) entre 21 K y temperatura ambiente y diversas técnicas magnéticas como son medidas de susceptibilidad AC en función de la temperatura para distintas frecuencias del campo alterno y en presencia de un campo magnético DC, y ciclos de histéresis y medidas de magnetización en función de la temperatura enfriando con y sin campo magnético. Los resultados estructurales, hiperfinos y magnéticos obtenidos son discutidos en el marco de la distribución atómica del $\mathrm{Cu}$ en la estructura fcc y comparados con la información reportada en la bibliografía presentada en el Capítulo 5.

Los resultados de la cinética de molienda muestran que después de las $12 \mathrm{~h}$ de molienda el sistema alcanza un estado estacionario. La variación de los parámetros estructurales e hiperfinos a temperatura ambiente con el contenido de $\mathrm{Cu}$ junto con el cálculo de probabilidades de distribución de átomos en una red $f c c$ muestran la formación de una solución sólida FeMnCu substitucional y aleatoria. La información aportada por las curvas de susceptibilidad AC, el comportamiento de la magnetización en enfriado con campo y la evolución del campo hiperfino con la temperatura sugieren que el sistema se comporta como un conjunto de partículas interactuantes con las características de un vidrio de espín. La presencia de exchange bias y la dependencia de éste con el campo aplicado durante el enfriado, permiten modelar a las partículas como un núcleo ordenado magnéticamente y una superficie desordenada. 


\subsection{Descripción del estudio realizado}

En la primera parte de esta Tesis son presentados los resultados del estudio de la cinética de molienda, donde se investigan los efectos del tiempo de molienda sobre los parámetros estructurales e hiperfinos. En una segunda parte se presentan y discuten los resultados del efecto del contenido de $\mathrm{Cu}$ en las propiedades estructurares, hiperfinas y magnéticas de la aleación ternaria, empleando para ello las técnicas mencionadas a continuación.

La información aportada por DRX permitió determinar las fases presentes, parámetros de red de la fase mayoritaria y el tamaño de cristalita, y cómo éstos evolucionaban al variar el contenido de $\mathrm{Cu}$ en las muestras.

La posibilidad de realizar medidas de XAS en el Laboratorio Nacional de Luz Sincrotrón (LNLS) ubicado en Campinas, Brasil permitió conocer como se distribuían los átomos en la estructura $f c c$, es decir, si ésta era una distribución al azar o si existían regiones ricas en un material u otro, lo que podría suceder debido a las distintas entalpías de mezcla que poseen los elementos constituyentes entre si. Además de ésto se pudo determinar la variación de la distancia a primeros vecinos con la concentración de $\mathrm{Cu}$, y estudiar si existía oxidación de los metales en la aleación.

Los resultados de EM a temperatura ambiente brindaron información sobre el entorno atómico de los átomos de Fe, pudiendo de esta forma determinar, por medio de modelos de ajuste, parámetros que indiquen si existen gradientes de campo eléctrico producidos por una asimetría en la distribución de carga o desdoblamientos magnéticos debido a las fases con orden magnético presentes. La incorporación substitucional de los átomos de $\mathrm{Cu}$ en la estructura fcc-FeMn fue seguida mediante la evolución de los parámetros hiperfinos con el contenido de dicho aleante. Para completar el estudio hiperfino se realizaron medidas empleando un criógeno de ciclo cerrado de He en el rango de temperaturas entre $300 \mathrm{~K}$ y $21 \mathrm{~K}$. Las temperaturas características obtenidas en cada caso fueron comparadas posteriormente con las correspondientes a las medidas magnéticas realizadas.

La información obtenida mediante diversas técnicas de caracterización magnética, junto con distintos modelos en los que se estudian la evolución de los parámetros obtenidos, brindan un amplio panorama de la respuesta estructural y magnética del sistema. 


\subsection{Referencias}

${ }^{1}$ G.C. Hadjipanayis y G.A. Prinz (editores), en "Science and Technology of Nanostructured Magnetic Materials”, Vol. 259 de NATO Advanced Study Institute, Series B: Physics, Plenum Press, New York, (1991).

2 J.L. Dormann y D. Fiorani (editores), en "Magnetic Properties of Fine Particles", North-Holland, Amsterdam, (1992).

${ }^{3}$ M.N. Baibich, en “Magnetism, Magnetic materials and their applications”, F. Leccabue y V. Sagrado (editores), World Scientific, Singapur, (1996), pág. 69.

${ }^{4}$ R.W. Siegel; Annu. Rev. Mater. Sci. 21, (1991), pág. 559.

${ }^{5}$ M. Knobel, W.C. Nunes, L.M. Socolovsky, E. De Biasi, J.M.Vargas y J.C. Denardin; J. Nanosci. Nanotechnol. 8, (2008), pág. 2836.

${ }^{6}$ O. Petracic; superlattices and microstructures (2010), doi:10.1016/j.spmi.2010.01.009.

${ }^{7}$ C. Suryanarayana; Progress in Materials Science 46, 1-2 (2001), pág. 1.

${ }^{8}$ T.D. Shen, R.B. Schwarz y J.D. Thompson; J. App. Phys. 85, 8 (1999), pág. 4110.

${ }^{9}$ R.B. Goldfarb y C.E. Patton; Phys. Rev. B 24, 3 (1980), pág. 1360.

${ }^{10}$ S.S. Saxena, J. Tang, Y.S. Lee y C.J. O'Connor; J. App. Phys. 76, 10 (1994), pág. 6820.

${ }^{11}$ F.R. de Boer, R. Boom, W.C.M. Mattens, A.R. Miedema y A.K. Niessen, en "Cohesion in Metals, Transition Metal alloys”, North-Holland, Ámsterdam (1989), pág. 240.

${ }^{12}$ P. H. Shingu, K. N. Ishihara, K. Uenishi, J. Kuyama, B. Huan y S. Nasu, en "Solid State Powder Processing”, A. H. Clauer and J. J. de Barbadillo (Editores), The Minerals, Metals and Materials Society, (1990), pág. 21.

${ }^{13}$ C.A.M. Mulder, A.J. van Duyneveldt y J.A. Mydosh; Phys. Rev. B 23, 3 (1980), pág. 1384.

${ }^{14}$ G.E. Bacon, N. Cowlam y A.G. Self; J. Phys. F: Met. Phys. 3, (1973), pág. 6.

${ }^{15}$ C. Paduani y J.C. Krause; Phys. Rev. B 58, 1, (1998), pág. 175.

${ }^{16}$ D. Spišák y J. Hafner; Phys. Rev. B 61, 17, (2000), pág. 11569.

${ }^{17}$ J. Martínez, S.M. Cotes, A.F. Cabrera, J. Desimoni y A. Fernández Guillermet; Mat. Sci. Eng. A 408, 1-2 (2005), pág. 26.

${ }^{18}$ Y. Endoh y Y. Ishikawa; J. Phys. Soc. Jpn. 30, (1971), pág. 1614.

${ }^{19}$ M. Uhrmacher, A. Kulinska, Yu.V. Baldokhin. V.V. Tcherdyntsev, S.D. Kaloshkin, A. Maddalena y G. Principi; Hyperfine Interactions 136/137, 3-8 (2001), pág. 327.

${ }^{20}$ T.B. Massalski, en “Binary Alloy Phase Diagrams”, H. Okamoto, P. Subramanian y L. Kacprzak (editores), $2^{\text {da }}$ edición, Vol. 2, pág. 1724. 
${ }^{21}$ J. Restrepo, A.L. Morales, J.M. González, G.A. Pérez Alcázar, G. Medina, C.A. Barrero, J. Tobón, G. Pérez, O. Arnache, J.D. Betancur y M.A. Giraldo; Hyp. Int. 134, 1 (2001), pág. 199.

${ }^{22}$ S.J. Stewart, J.F. Marco, P. Crespo, J.J. Romero, A. Martínez, A Hernando, F.J. Palomares y J.M. González; J. Nanosci. Nanotechnol. 7, 2 (2007), pág. 610.

${ }^{23}$ J. Restrepo, J.M. Grenèche, A. Hernando, P. Crespo, M.A. García, F.J. Palomares y J.M. González; J. Magn. Magn. Mat. 290-291, (2005), pág. 602.

${ }^{24}$ J. Restrepo, J.M. Grenèche y J.M. González; Physica B: Condensed Matter 354, 1-4 (2004), pág. 174.

${ }^{25}$ M. Mizrahi, F.A. Cabrera, S.J. Stewart, H.E. Troiani, S.M. Cotes y J. Desimoni; Physica B 354, 1-4 (2004), pág. 133.

${ }^{26}$ M. Mizrahi, S.J. Stewart, A.F. Cabrera y J. Desimoni; Hyp. Int. 161, 1-4 (2005), pág. 171.

${ }^{27}$ M. Mizrahi, A.F. Cabrera y J. Desimoni; Hyp. Int. 179, 1-3 (2007), pág. 39.

${ }^{28}$ M. Mizrahi, A.F. Cabrera y J. Desimoni; J. Alloys Comp. (2009) (en prensa), versión on-line: http://dx.doi.org/10.1016/j.jallcom.2009.10.166.

${ }^{29}$ M. Mizrahi, A.F. Cabrera y J. Desimoni; J. Appl. Phys. 107, 4 (2010), pág. 044311. 


\section{Capítulo 2. Antecedentes}

En este Capítulo se brindará un resumen de los trabajos más significativos realizados en los sistemas FeMn, FeCu, CuMn y FeMnCu. Se presentan los resultados relacionados, principalmente, con las propiedades estudiadas en esta Tesis y cómo los mismos fueron interpretados por los diferentes autores.

\subsection{Sistema FeMn}

El sistema FeMn ha sido estudiado por varios autores, principalmente por sus propiedades en la metalurgia, ${ }^{1,2}$ efectos de memoria de forma ligados a la transformación martensítica ${ }^{3}$ y como capa de "anclaje” en dispositivos multicapas en válvulas de spin, ${ }^{4,5}$ entre otras cosas. La entalpía de mezcla de este sistema muestra un amplio rango de valores según lo encontrado en bibliografía, ${ }^{6}$ que va desde $1 \mathrm{~kJ} / \mathrm{mol} \mathrm{a}-9 \mathrm{~kJ} / \mathrm{mol}$. Desde el punto de vista magnético, el Fe es un metal ferromagnético con una temperatura de Curie de $T_{C}=1043 \mathrm{~K}^{7}$ y el $\mathrm{Mn}$ es un metal antiferromagnético con una temperatura de Néel de $T_{N}=95 \mathrm{~K}^{7}$. En la figura 2.1 se muestra el diagrama de fases del sistema FeMn según Massalski. ${ }^{8}$ 


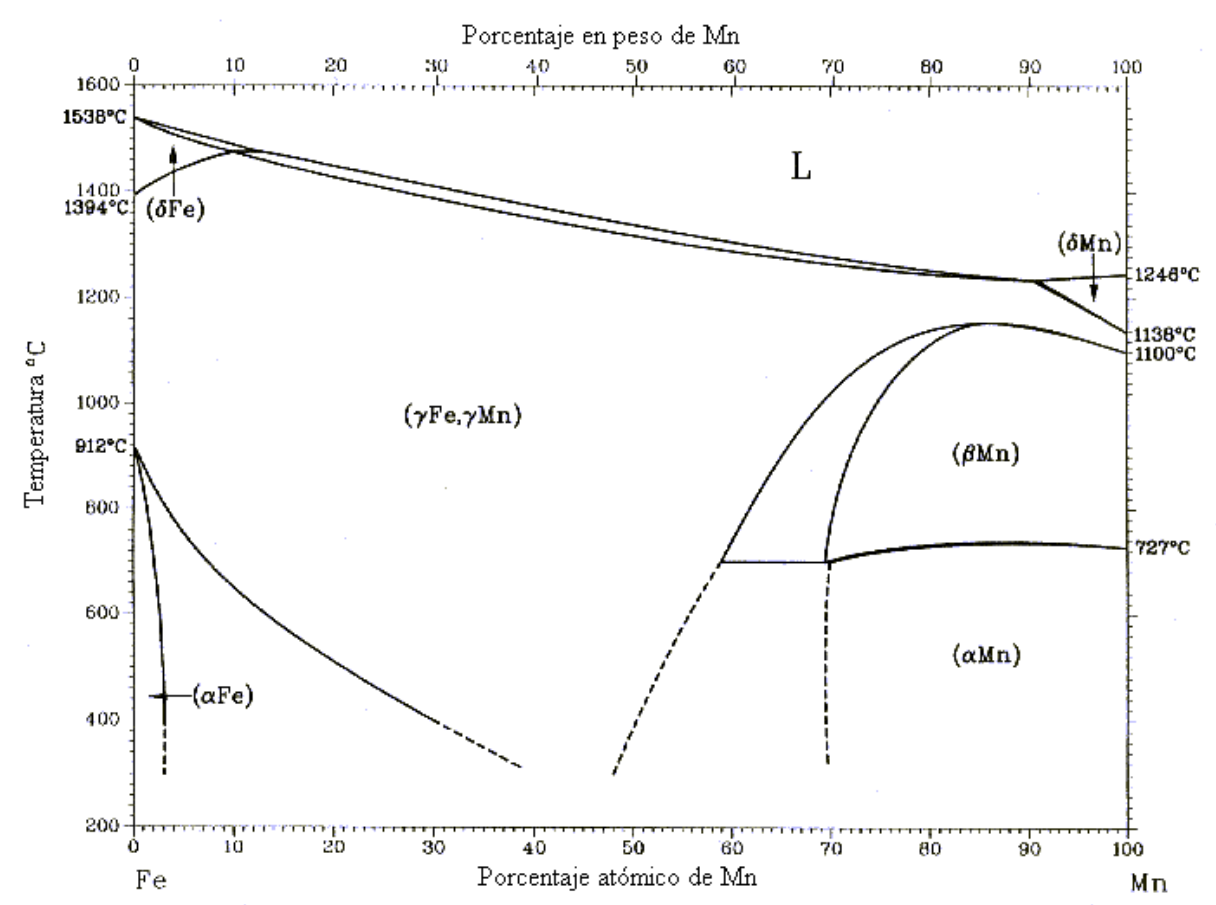

Figura 2.1: Diagrama de fases del sistema FeMn en función del porcentaje de Mn.

En la figura 2.1 se pueden observar las distintas fases presentes en el sistema, a saber:

o La solución sólida $\alpha-\mathrm{Fe}(\mathrm{Mn})$, la cual tiene una estructura cúbica centrada en el cuerpo $(b c c)$.

o La solución sólida $\gamma$-FeMn. Esta posee una estructura cúbica centrada en las caras ( $f c c)$ en la cual los átomos de Mn pueden reemplazar hasta un 70 \% de los átomos de Fe.

o $\quad \delta$-Fe (bcc-Fe a altas temperaturas).

o La fase $\gamma$-Mn posee una estructura $f c c$.

o Las fases $\beta-M n$ y $\alpha-M n$ poseen estructuras cúbicas complejas.

Bajo tratamientos térmicos se puede inducir la transformación de fase en el sistema binario FeMn que ocurre entre las estructuras cristalinas hexagonal compacta denominada $\varepsilon$ o martensita y la $f c c$, denominada $\gamma$ o austenita. Es por esto que a esta transformación no difusiva se la denomina martensítica, no habiendo cambio en la composición química antes y después de la transformación. 


\subsubsection{Trabajos previos}

El trabajo de Umebayashi e Ishikawa ${ }^{9}$ es uno de los primeros antecedentes en el estudio del sistema FeMn. Muestras con una concentración de Mn entre 18 \%at. y 49 \%at. preparadas en un horno de inducción fueron caracterizadas por difracción de neutrones y medidas de susceptibilidad magnética. Se encontró que la fase mayoritaria era de estructura $f c c$ con la presencia de una mínima fracción de hcp. Los autores mencionan que la variación de la fracción de esta última está relacionada con el proceso de enfriado de las muestras y el pulido superficial al que posteriormente fueron sometidas. Paduani et. al., en un primer trabajo, ${ }^{10}$ estudiaron muestras de FeMn preparadas por fusión en horno de arco con contenidos de Mn de hasta un 17.5 \%at. La estructura encontrada en todas las muestras fue $b c c$ con un parámetro de red que mostraba un leve incremento a medida que el contenido de Mn aumentaba. En un segundo trabajo ${ }^{11}$ los autores extendieron el rango composicional de estudio hasta un 50 \%at. de Mn. A diferencia del trabajo anterior, aquí se agregó un 0.2 \%at. de C en las muestras con un contenido inferior al 20 \%at. de Mn, para estabilizar la fase $f c c$. Los resultados estructurales mostraron una única fase $f c c$ con un parámetro de red que permanece invariante hasta una concentración de 27 \%at. de Mn y luego comienza a aumentar linealmente, con una variación del $1 \%$ hasta llegar a la concentración más alta. Martínez y otros ${ }^{12}$ estudiaron muestras de FeMn entre 17 \%at. y 31 \%at. de Mn preparadas en horno de fusión de arco. Los resultados de difracción de neutrones indican la presencia de las fases bcc-FeMn, fccFeMn y hcp-FeMn. El parámetro de red de la fase $f c c$ encontrado aumenta con el contenido de Mn desde $3.5915 \AA$ a $3.6092 \AA$. Endoh e Ishikawa ${ }^{13}$ fueron de los primeros en estudiar el sistema FeMn preparado por aleado mecánico (AM) en todo su rango composicional. Encontraron que, a partir de 15 \%at. de Mn, la fase principal posee estructura $f c c$ con un parámetro de red de $3.617 \AA$ aumentando hasta $3.640 \AA$ para un 60 \%at. de Mn. Trabajos más recientes ${ }^{14}$ mostraron que existe un incremento de la región en la que se presenta la fase $f c c$-FeMn en muestras preparadas por AM, llegando a formarse dicha fase para concentraciones de Mn desde $14 \%$ at. hasta un $70 \%$ at.

En lo que respecta al estudio del orden magnético que presentan estas aleaciones Fujimori es uno de los pioneros. ${ }^{15}$ En su trabajo reporta los resultados obtenidos en tres muestras de fcc-FeMn con concentraciones de 30 \%at., 35 \%at. y 40 \%at. de Mn, preparadas por fusión en horno de arco. Encuentra cambios en las medidas de susceptibilidad magnética y dilatometría, en función de la temperatura. Estos cambios fueron asociados con la transición paramagnética-antiferromagnética del sistema. Encuentra también que la temperatura de Néel asociada a este proceso es del orden de $423 \mathrm{~K}$ para la muestra con 30 \%at. de Mn aumentando hasta $453 \mathrm{~K}$ para la muestra con 40 \%at. de Mn. Umebayashi e Ishikawa ${ }^{9}$ realizaron ${ }^{9}$ más atrás un estudio magnético similar al presentado por 
Fujimori, ${ }^{15}$ encontrando que la temperatura de Néel de la fase $f c c$-FeMn variaba entre 360 K y 502 K para concentraciones de Mn entre 18.5 \%at. y 49.2 \%at., respectivamente. Un par de años más tarde, Endoh e Ishikawa ${ }^{13}$ completaron la evolución de la temperatura de orden con la concentración de Mn. En este caso se emplearon resultados obtenidos de difracción de neutrones y susceptibilidad magnética, donde encontraron que en todo el rango composicional estudiado las aleaciones de FeMn se ordenan antiferromagnéticamente. También observaron que la temperatura de Néel es dependiente de la concentración de Mn, siendo ésta más elevada a mayores concentraciones. Paduani et. al. ${ }^{11}$ encontraron por medidas de calor específico una dependencia de la temperatura de Néel con el contenido de Mn similar a la reportada por Endoh e Ishikawa. ${ }^{13}$ Finalmente, Martínez y otros ${ }^{16}$ obtuvieron una expresión empírica para la dependencia de la temperatura de Néel de la fase $f c c$-FeMn con el contenido de Mn, la cual está dada por:

$$
T_{N}^{f c c}(K)=67 \cdot x_{F e}+540 \cdot x_{M n}+\frac{X_{F e} X_{M n}}{3}\left[2282+2068\left(x_{F e}-x_{M n}\right)\right]
$$

donde $x_{F e} \mathrm{y} x_{M n}$ son las fracciones atómicas de Fe y Mn, respectivamente.

Un estudio completo de las propiedades hiperfinas del sistema FeMn fue realizado posteriormente por Paduani y otros. ${ }^{11}$ Los espectros medidos a temperatura ambiente fueron reproducidos con una distribución de desdoblamientos cuadrupolares para las muestras con un contenido menor al 19 \%at. de Mn, mientras que para las concentraciones mayores se empleó una distribución de campos magnéticos hiperfinos basada en el orden antiferromagnético que presenta el sistema. El valor del campo hiperfino medio a temperatura ambiente (TA) para la muestra con 23 \%at. de Mn fue de aproximadamente $2 \mathrm{~T}$, mostrando éste un incremento con el contenido de Mn. Para la misma concentración, el campo hiperfino medio en la medida realizada a $77 \mathrm{~K}$ aumentó a 3.3 T. Martínez y otros ${ }^{17}$ estudiaron los parámetros hiperfinos en muestras preparadas en horno de arco con contenidos de Mn entre 15 \%at. y 30 \%at. Los espectros fueron reproducidos empleando dos interacciones correspondientes a la fase $h c p$ y a la fase $f c c$, respectivamente. Ya que la fase $f c c-F e M n$ posee un orden antiferromagnético a TA en todo el rango de concentraciones estudiado, la interacción hiperfina asociada a esta fase fue un sextete no resuelto con un valor de $2.3 \mathrm{~T}$ a $2.7 \mathrm{~T}$ y un corrimiento isomérico entre $-0.05 \mathrm{~mm} / \mathrm{s}$ y $-0.09 \mathrm{~mm} / \mathrm{s}$. Cherdyntsev ${ }^{18}$ presentó resultados Mössbauer en muestras preparadas por AM con concentraciones entre 10 \%at. y 90 \%at. de Mn. Para las muestras con contenidos entre 20 \%at. y 70 \%at., propuso un ajuste con un sextete no resuelto más una línea simple, obteniendo para la muestra de menor concentración un campo hiperfino de $2.1 \mathrm{~T}$, similar al encontrado anteriormente por Paduani. ${ }^{11} \mathrm{El}$ valor del corrimiento isomérico de la contribución magnética y el de la línea simple resultó ser semejante, siendo del orden de $-0.08 \mathrm{~mm} / \mathrm{s}$. Uhrmacher y otros ${ }^{19}$ estudiaron el comportamiento de los parámetros 
hiperfinos del sistema FeMn preparado por aleado mecánico, con concentraciones de Mn entre 10 \%at. y 90 \%at. Para las muestras con un contenido menor al 15 \%at. de Mn los espectros Mössbauer presentan un sextete magnético y una leve contribución en forma de línea simple en la parte central del espectro. La componente magnética resuelta fue reproducida empleando tres sitios magnéticos correspondientes a entornos del átomo de Fe con cero, uno y dos o más átomos de Mn primeros vecinos en la fase $b c c$. La componente central fue asociada con la fase $f c c-\mathrm{Fe}(\mathrm{Mn})$ y ajustada con un sextete no resuelto de bajo campo hiperfino. Para las concentraciones con contenidos de Mn entre 20 \%at. y 70 \%at. los espectros presentan solamente la contribución central antes mencionada, la cual se va ensanchando a medida que la concentración de Mn aumenta. Como en la región anterior esta contribución fue reproducida con un sextete no resuelto de $2.8 \mathrm{~T}$ de campo hiperfino para la muestra con un 40 \%at. de Mn. Para las concentraciones de Mn mayores al 40 \%at. los espectros consisten en una línea simple ancha o en un doblete no resuelto asociados con la fase $b c c-\mathrm{Mn}(\mathrm{Fe})$. 


\subsection{Sistema $\mathrm{FeCu}$}

El sistema $\mathrm{FeCu}$ ha sido estudiado desde principios de la década del '80 desde el punto de vista de investigación básica, siendo a mediados de la década del '90 cuando el sistema recobró importancia por descubrirse que el mismo presentaba efectos magnetorresistivos. ${ }^{20} \mathrm{Al}$ tener una entalpía de mezcla positiva $(13 \mathrm{~kJ} / \mathrm{mol})^{21}$ las solubilidades entre $\mathrm{Fe}$ y $\mathrm{Cu}$ son muy pequeñas (3.5 \%at. a $1096{ }^{\circ} \mathrm{C}$ para Fe en $\mathrm{Cu}$ y 6.7 \%at. para $\mathrm{Cu}$ en Fe), ${ }^{21}$ por lo tanto, es sólo posible obtener una mezcla de estos elementos empleando métodos fuera del equilibrio termodinámico, como el enfriado rápido desde el estado líquido (fast quenching) ${ }^{22}$ o evaporación y posterior deposición sobre un sustrato frío (sputtering o molecular beam epitaxy $M B E$ ) ${ }^{23}$ Desde hace algunas décadas se ha probado que también es posible unir ambos materiales por técnicas de soldado en frío como lo es el aleado mecánico. ${ }^{24}$

En la figura 2.2 se muestra el diagrama de fases del sistema FeCu según Swartzendruber. ${ }^{25}$

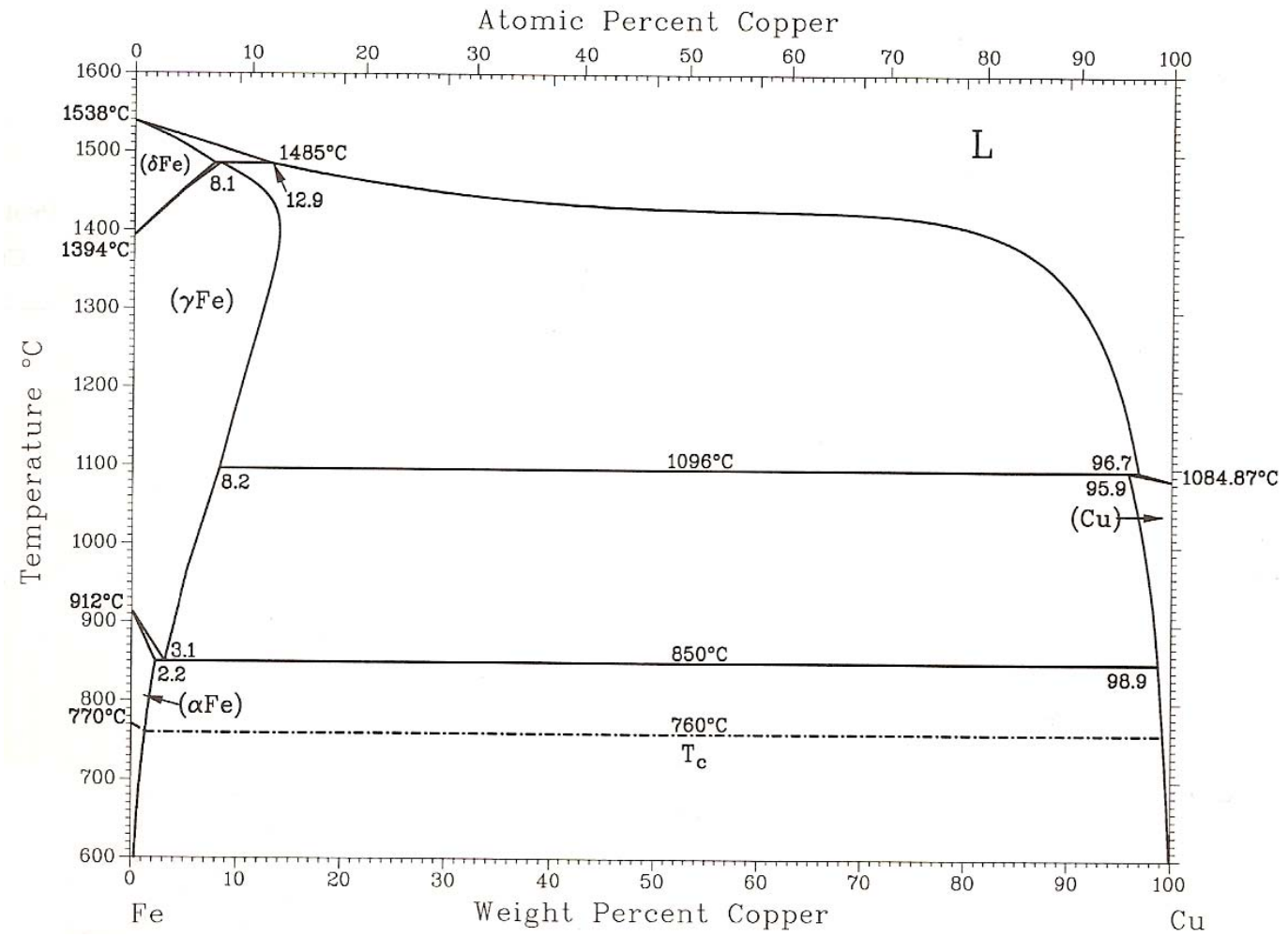

Figura 2.2: Diagrama de fases del sistema FeCu función de la fracción de Fe. 
En la figura 2.2 se pueden observar las distintas fases presentes en el sistema, a saber:

o La solución sólida $\alpha-\mathrm{Fe}(\mathrm{Cu})$ de baja temperatura, con una estructura bcc y presente entre 0 \%at. y 1.9 \%at. de $\mathrm{Cu}$.

o La solución sólida $\delta$ - $\mathrm{Fe}(\mathrm{Cu})$ de alta temperatura, posee una estructura bcc y está presente entre 0 \%at. y 6.7 \%at. de $\mathrm{Cu}$.

o La solución sólida $\gamma$-Fe(Cu), la cual tiene una estructura $f c c$ y está presente entre $0 \%$ at. y 6.7 \%at. de $\mathrm{Cu}$.

o La fase $\mathrm{Cu}$ de estructura $f c c$ que se presenta entre $96.5 \%$ at. y $100 \%$ at. de Cu.

\subsubsection{Trabajos previos}

En esta sección se presentarán los resultados más importantes obtenidos sobre el sistema FeCu relacionados con la esta Tesis.

Chien y otros $^{26}$ fueron los primeros en estudiar el sistema $\mathrm{FeCu}$ en todo su rango composicional. Si bien las muestras fueron preparadas por sputtering y no por AM, su estudio brindó un aporte significativo a esta Tesis. Los resultados de difracción de rayos $\mathrm{X}$ mostraron que la estructura $f c c$ es la mayoritaria para concentraciones menores a 70 \%at. de $\mathrm{Fe}$, siendo la estructura $b c c$ la predominante para contenidos de Fe mayores a 80 \%at. El grupo de Shingu ${ }^{27,28}$ fue uno de los primeros en estudiar el sistema $\mathrm{FeCu}$ en todo el rango de concentraciones preparado por AM. Ellos encontraron que el sistema poseía una estructura $f c c$ hasta aproximadamente un 60 \%at. de Fe y bcc para concentraciones de Fe superiores al 80 \%at. Observaron que el parámetro de red en la fase $f c c$ era más grande que el esperable según la ley de Vegard, ${ }^{29}$ y que variaba con la concentración de Cu. En una serie de trabajos Eckert et. al. ${ }^{30,31}$ estudiaron por DRX la evolución de los parámetros estructurales con el tiempo de molienda y temperatura del tratamiento térmico posterior. Confirmaron la existencia de las fases $f c c$ y $b c c$ ( $\mathrm{Fe}<60$ \%at. y $\mathrm{Fe}>80$ \%at., respectivamente) y la coexistencia de ambas fases en la región intermedia. En su primer trabajo Eckert y colaboradores ${ }^{30}$ mostraron que al calentar las muestras se separaban las fases casi sin crecimiento del tamaño de grano. Una vez alcanzada la separación total de las fases, el tamaño de grano comenzaba a aumentar considerablemente. En este mismo trabajo se determinó que el tamaño de grano en la fase $f c c$ está entre $9 \mathrm{~nm}$ y $20 \mathrm{~nm}$. El grupo de Yavari ${ }^{32}$ reportó la fabricación de una solución sólida $f c c$ de FeCu producida por AM. Ellos realizaron medidas de DRX y observaron que las intensidades relativas de las líneas de difracción del Fe y del Cu permanecían casi constantes al comienzo de la molienda. A partir de las $50 \mathrm{~h}$ de molienda el tamaño de cristalita alcanzaba un valor estacionario de aproximadamente $10 \mathrm{~nm}$ y las líneas correspondientes a la fase 
bcc-Fe comenzaban a desaparecer hasta no ser distinguibles. Ma, Atzmon y Pinkerton ${ }^{33}$ investigaron las propiedades estructurales y termodinámicas en función de la concentración de $\mathrm{Cu}$ en muestras preparadas en un molino de alta energía. Los resultados de las medidas estructurales son similares a los reportados en los trabajos previos. ${ }^{31}$ En otro trabajo ${ }^{34}$ se presentó un estudio del sistema $\mathrm{Fe}_{\mathrm{x}} \mathrm{Cu}_{1-\mathrm{x}}(\mathrm{x}=0.1$ a 0.7$)$ también preparado por $\mathrm{AM}$. Los autores encuentran que la fase de estructura $f c c$ se extiende desde $\mathrm{x}=0.1$ hasta $\mathrm{x}=0.5$, mientras que para contenidos de Fe mayores la fase principal tiene estructura $b c c$. Los tamaños de partícula que determinan a partir de DRX van desde 13 a $19 \mathrm{~nm}$. Huang, He y Wu publicaron un trabajo ${ }^{35}$ donde exhiben fotografías de microscopía de transmisión electrónica de alta resolución en muestras de fcc-FeCu preparadas por molienda mecánica. En éstas es posible ver que en las zonas de soldadura hay cristales muy pequeños y zonas nanométricas con estructura amorfa; concluyen que el mezclado se produce por interdifusión en estado sólido y que la elevación de la energía libre del sistema se debe al almacenamiento de energía en los defectos de la red y en la energía interfacial. Jiang y colaboradores ${ }^{36}$ hallaron que el parámetro de red de la fase $f c c-\mathrm{Fe}_{50} \mathrm{Cu}_{50}$ obtenido por DRX se incrementaba con el tiempo de molienda desde un valor de $3.615 \AA$ hasta $3.645 \AA$, lo cual fue atribuido a efectos de magnetovolumen. Además, mostraron que la región de concentraciones donde se presentan las distintas fases cristalográficas es fuertemente dependiente del método de preparación de las muestras.

En lo que respecta a la caracterización hiperfina del sistema $\mathrm{FeCu}$ el trabajo de Chien ${ }^{26}$, previamente citado, mostró por medidas de EM, que el campo magnético hiperfino aumenta, mientras que el corrimiento isomérico disminuye al aumentar la concentración de Fe. Encontró que la temperatura de Curie aumenta con el contenido de Fe de las muestras, teniendo una salto a aproximadamente 70 \%at. de Fe donde la fase bcc comienza a ser la mayoritaria. Crespo et. al. ${ }^{34}$ presentaron resultados de $\mathrm{EM}$ a temperatura ambiente en aleaciones $\mathrm{Fe}_{\mathrm{x}} \mathrm{Cu}_{1-\mathrm{x}}$ preparadas por $\mathrm{AM}$. En las concentraciones $\mathrm{x}=0.5 \mathrm{y} \mathrm{x}=0.7$ se observó un espectro magnético con valores del campo hiperfino medio de $21 \mathrm{~T}$ y $28 \mathrm{~T}$, y corrimientos isoméricos de $0.11 \mathrm{~mm} / \mathrm{s}$ y $0.07 \mathrm{~mm} / \mathrm{s}$, respectivamente. Otro estudio empleando EM fue el realizado por Macri y colaboradores ${ }^{37}$ en una muestra de $\mathrm{Fe}_{50} \mathrm{Cu}_{50}$ preparada por molienda. Los autores encuentran la presencia de tres sitios distintos para el Fe: uno representado por un sextete ensanchado de campo magnético hiperfino igual a $22 \mathrm{~T}$ y un corrimiento isomérico de $0.09 \mathrm{~mm} / \mathrm{s}$ atribuida a la solución sólida $\mathrm{Fe}_{50} \mathrm{Cu}_{50}$, otro sitio representado por un doblete con un valor del desdoblamiento cuadrupolar de $0.95 \mathrm{~mm} / \mathrm{s}$ y un corrimiento isomérico de $0.13 \mathrm{~mm} / \mathrm{s}$ asociado a una región rica en $\mathrm{Cu}(f c c-\mathrm{Cu}(\mathrm{Fe})$ ), y por último una línea simple con corrimiento isomérico de $-0.04 \mathrm{~mm} / \mathrm{s}$ proveniente de la fase $f c c$-Fe. El estudio de Jiang ${ }^{36}$ mostró que los espectros Mössbauer medidos a temperatura ambiente presentan, para tiempos de molienda cortos (30 minutos), una contribución mayoritaria, sextete de campo cercano a los $33 \mathrm{~T}$, atribuido a $\alpha$-Fe. A medida que el tiempo de molienda se incrementa el sextete anterior 
pasa a tener valores cercanos a $30 \mathrm{~T}$ para $10 \mathrm{~h}$ de molienda y una pequeña contribución no magnética se comienza a observar en la región central del espectro. La primera de las contribuciones mencionadas anteriormente fue asociada a regiones mayoritarias de $\alpha$-Fe con uno o dos átomos de $\mathrm{Cu}$ como primeros vecinos; la segunda de las contribuciones, un doblete, es atribuido a precipitados de $f c c$-Fe en una matriz de $\mathrm{Cu}$.

Con respecto al momento magnético por átomo Shingu y colaboradores ${ }^{27}$ observaron que no existen grandes variaciones en la región de concentraciones ricas en Fe y sí las hay para las composiciones con porcentaje mayoritario de $\mathrm{Cu}$, donde éste decrece hasta hacerse nulo alrededor de un 85 \%at. de Cu. Yavari et. al. ${ }^{32}$ publicaron una medida de magnetización en función de la temperatura donde se puede observar una caída de la misma con el aumento de la temperatura, haciéndose nula a $500 \mathrm{~K}$, para luego comenzar a aumentar hasta llegar a la magnetización correspondiente al $\alpha$-Fe. En este trabajo también se presentan ciclos de histéresis medidos a varias temperaturas. Del ajuste de los ciclos encuentran un comportamiento anómalo en el campo coercitivo, teniendo éste un mínimo al bajar la temperatura y alcanzando valores de casi 1000 Oe para una temperatura de $10 \mathrm{~K}$. Un resultado interesante fue el presentado por el grupo de Chien, ${ }^{38}$ donde se vio que el campo coercitivo aumenta con el tiempo de molienda, hasta llegar a un valor máximo, el cual correspondería al momento en que la fase bcc comienza a desaparecer por DRX. Un grupo australiano de investigadores presenta en 1995 dos trabajos ${ }^{39,40}$ acerca de las propiedades magnéticas de las aleaciones FeCu. Allí los autores explican el comportamiento magnético de la aleación por la presencia de clusters magnéticos que interactúan entre sí y que serían superparamagnéticos. Esos clusters serían regiones ricas en Fe.

Hacia finales de 1994 fue publicado el primer trabajo sobre el efecto magnetorresistivo gigante en aleaciones de $\mathrm{FeCu}$ preparadas por $\mathrm{AM}^{41}$ Los autores observan que el efecto magnetorresistivo es mayor cuando menor es la temperatura de medida. Realizaron tratamientos térmicos de las muestras a $573 \mathrm{~K}$, los cuales producen un aumento importante en la magnetorresistencia del material que, a su vez, es disminuida en tratamientos a temperaturas más elevadas. Otro grupo estudió la magnetización y magnetorresistencia explorando un rango entre 10 \%at. y 90 \%at. de Fe, tanto en muestras con y sin tratamiento térmico preparadas por $\mathrm{AM}^{42}$ Observaron que la magnetización de la muestra se hace nula para una concentración de 20 \%at. de Fe. Para la muestra sin tratamiento térmico el máximo en el efecto magnetorresistivo se observó para la concentración de un 30 \%at. de Fe el cual se corre a un 25 \%at. de Fe cuando la muestra es tratada térmicamente. También encontraron que el sistema presenta un comportamiento tipo vidrio de espín reentrante para concentraciones entre 18 \%at. y 28 \%at. de Fe. 


\subsection{Sistema CuMn}

El sistema CuMn obtuvo su mayor interés con el descubrimiento de algunas propiedades magnéticas que éste presentaba, las cuales no pudieron ser explicadas pensando al sistema como un conjunto de átomos magnéticos con un orden de largo alcance. A esta nueva fase magnética se la denominó vidrio de espín (spin glass).

La entalpía de mezcla de este sistema tiene un valor negativo, ${ }^{43}$ por este motivo la obtención de una solución sólida de estos elementos puede ser conseguida por diversas técnicas como se mostrará en la sección de antecedentes del sistema. La figura 2.3 muestra el diagrama de fases del sistema CuMn. ${ }^{44}$

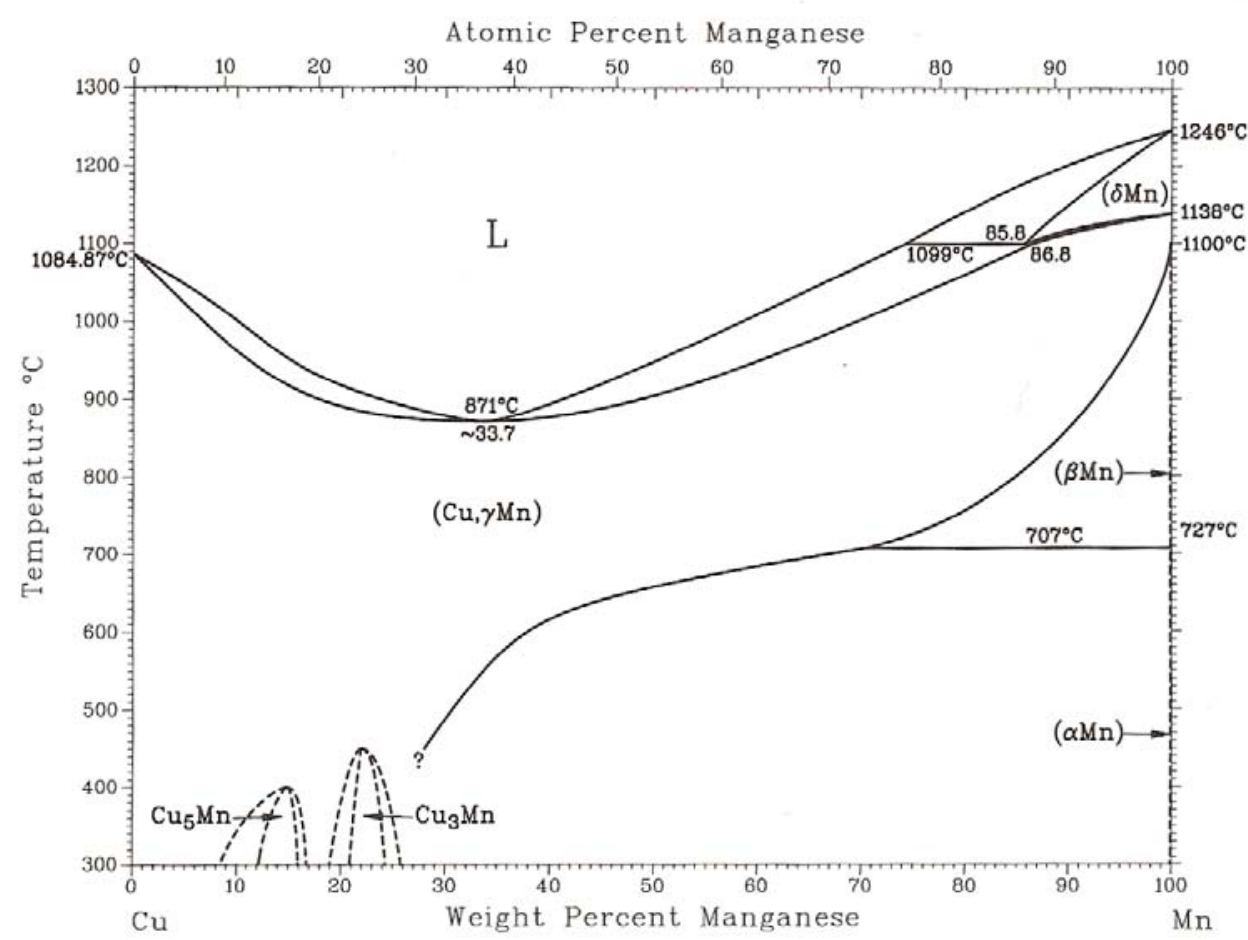

Figura 2.3: Diagrama de fases del sistema CuMn en función del porcentaje de Mn.

En la figura 2.3 se pueden observar las distintas fases presentes en el sistema, a saber:

o La fase $(\mathrm{Cu}, \gamma-\mathrm{Mn})$ con estructura $f c c$ y puede ser encontrada en todo el rango de $\mathrm{Mn}$.

o La solución sólida $\delta-\mathrm{Mn}(\mathrm{Cu})$ de alta temperatura posee una estructura $b c c$ y está presente entre 87.5 \%at. y 100 \%at. de Mn.

o Las fases $\beta$-Mn y $\alpha$-Mn poseen estructuras cúbicas complejas, existentes sólo para 100 \%at. de Mn. 


\subsubsection{Trabajos previos}

Las propiedades estructurales del sistema CuMn fueron investigadas por Meneghetti ${ }^{45}$ en un rango composicional desde 11 \%at. a 85 \%at. de Cu. Encontraron por DRX que hasta un 69 \%at. de $\mathrm{Cu}$ la única fase presente es la $f c c$, mientras que para concentraciones mayores aparece una fase tetragonal centrada en las caras. El parámetro de red de la fase $f c c$ aumenta con el contenido de $\mathrm{Cu}$ desde un valor de $3.61 \AA$ hasta $3.75 \AA$.

Uno de los pocos trabajos abocados al estudio de las propiedades hiperfinas del sistema CuMn fue llevado a cabo por Window. ${ }^{46}$ En el mismo dopan con $1 \%$ peso de ${ }^{57} \mathrm{Fe}$ diversas muestras de CuMn preparadas en horno de arco, abarcando un rango de concentraciones de 0 \%at. a 90 \%at. de Mn. Las muestras fueron caracterizadas por EM en el rango de temperaturas que va desde $300 \mathrm{~K}$ hasta $4.2 \mathrm{~K}$. Los resultados de las medidas realizadas a temperatura ambiente mostraron una estructura de dobletes, con corrimientos isoméricos que decrecen desde $0.22 \mathrm{~mm} / \mathrm{s}$ a $-0.05 \mathrm{~mm} / \mathrm{s}$ a medida que el contenido de Mn aumenta desde 5 \%at. hasta 90 \%at. A $4.2 \mathrm{~K}$, una estructura magnética muy distribuida comienza a resolverse por lo cual el autor propuso una distribución de campos magnéticos hiperfinos para reproducir los espectros. Los valores para el campo hiperfino medio obtenidos están en el rango de $12 \mathrm{~T}$ hasta $0.5 \mathrm{~T}$ para las muestras con 5 \%at. y 90 \%at. de Mn, respectivamente. El corrimiento isomérico de la distribución magnética evoluciona hacia valores más grandes con la disminución de la concentración de Mn, comportamiento que se mantiene en toda la región de temperaturas estudiadas. En este trabajo también se estimaron las temperaturas de orden del sistema haciendo barridos térmicos a velocidad constante, obteniéndose temperaturas entre 50 K y 200 K para las muestras con 10 \%at. y 50 \%at. de Mn, respectivamente. Para las concentraciones más altas de Mn no fue posible hacer esta determinación debido a la gran variación del corrimiento isomérico para esas muestras. Finalmente, el autor menciona que la incorporación del Mn favorece la solubilidad del Fe en la matriz de CuMn, pero que existe una tendencia a la formación de aglomerados de MnFe sin presencia de $\mathrm{Cu}$.

El primer trabajo donde se formuló una teoría para explicar el comportamiento de los vidrios de espín en el sistema CuMn fue escrito por Edwards y Anderson. ${ }^{47}$ En éste se brindaba un modelo teórico que explicaba el comportamiento de cúspide observado en la susceptibilidad magnética en muestras de Mn diluido en Cu preparadas por AM. El argumento en que se basa la teoría es que, a pesar de no existir un orden ferromagnético o antiferromagnético, por causa de las interacciones de carácter oscilante entre espines, existe un estado fundamental en el que los espines están alineados, aún en una dirección que puede ser aleatoria. A una determinada temperatura, conocida como la temperatura de congelamiento del sistema $\left(T_{f}\right)$, la existencia de esas direcciones preferenciales conducirán a un comportamiento colectivo de los espines dando lugar al máximo que aparece en la 
curva de susceptibilidad. Mulder y colaboradores ${ }^{48}$ mostraron nuevos resultados relacionados con el comportamiento de vidrio de espín que poseen las aleaciones de CuMn. En este trabajo los autores emplearon las técnicas de molienda mecánica y horno de arco para obtener las muestras. Con estas técnicas prepararon aleaciones de CuMn con un porcentaje de Mn entre 0.23 \%at. y 6.3 \%at. A estas preparaciones les realizaron medidas de susceptibilidad AC en función de la temperatura, la frecuencia del campo excitante y en presencia de diversos campos estáticos externos. Los resultados de las medidas mostraron la existencia de una cúspide muy pronunciada a temperaturas del orden de las decenas de grados K. Mencionan que, dentro de la precisión de sus experimentos, la temperatura característica observada en las muestras con menor concentración de Mn es independiente de la frecuencia del campo AC entre $1 \mathrm{~Hz}$ y $10 \mathrm{kHz}$. Las muestras con un contenido mayor al 1 \%at. de Mn presentan una leve variación de la posición del máximo de 0.23 $\mathrm{K}$ a $0.49 \mathrm{~K}$ para frecuencias con 3 órdenes de magnitud de diferencia. Para temperaturas superiores a la temperatura del máximo de la susceptibilidad no se observa variación alguna con la frecuencia, colapsando todas en curvas con un comportamiento tipo Curie-Weiss, extendiéndose hasta altas temperaturas $(150 \mathrm{~K})$. La dependencia del máximo con la frecuencia de medida no pudo ser reproducida empleando una ley de tipo Arrhenius, ya que los valores obtenidos tanto para la energía de activación como para la frecuencia propia del sistema no poseen significado físico. La ley de Volguel-Fulcher ${ }^{49}$ fue propuesta para solucionar este problema, ya que la misma contempla la presencia de interacciones entre las partículas del sistema mediante la incorporación de un parámetro fenomenológico $T_{0}$. Con este modelo los valores obtenidos para los parámetros ajustados fueron realistas, aunque un significado del parámetro $T_{0}$ no fue brindado. Por otra parte, la presencia de un campo magnético DC externo origina que la intensidad del pico de la susceptibilidad se vea reducida en un $15 \%$ para un campo de 500 Oe. Este efecto es más notorio después de un tratamiento térmico seguido de un enfriado rápido. Así como la dependencia con la frecuencia los cambios son más notorios en las cercanías del máximo, para otras regiones las curvas de susceptibilidad colapsan a una única.

Banerjee y Majumdar, ${ }^{50}$ en 1992, realizaron un estudio magnético similar al presentado por Mulder ${ }^{48}$ pero extendieron el rango de concentraciones estudiadas desde $4.4 \%$ at. hasta $83 \%$ at. de Mn. Las muestras fueron preparadas en un horno de inducción y posteriormente algunas fueron tratadas térmicamente. Las medidas de susceptibilidad AC mostraron un comportamiento de cúspide de la componente en fase de la susceptibilidad, tal como el esperado en un sistema tipo vidrio de espín. Este comportamiento fue observado para todo el rango composicional estudiado, encontrándose la temperatura del máximo a valores mayores a medida que el contenido de Mn aumentaba. Sin embargo, la forma redondeada de la cúspide para las muestras con alto contenido de Mn sugiere que es más apropiada una descripción en términos de una distribución de partículas superparamagnéticas. Por este motivo, los autores usaron una distribución de barreras de 
anisotropías basadas en la teoría de Néel ${ }^{51}$ para describir el comportamiento de los clusters. Para comprobar la validez de su hipótesis, propusieron realizar medidas de susceptibilidad AC en presencia de campos estáticos. Observaron en las muestras con 36 \%at. y 46 \%at. de Mn que la intensidad de la curva de susceptibilidad vs. T disminuye significativamente con la presencia de un campo DC, aun para temperaturas lejanas a la temperatura del máximo. Concluyen que la disminución observada para todas las temperaturas es un indicativo de orden de corto alcance. Finalmente, concluyeron que los comportamientos observados para las muestras con bajos contenidos de Mn, menores que el 10 \%at., se encuentran dentro de los esperados para vidrios de espín. Concentraciones intermedias, entre 10 \%at. y 55 \%at. de Mn, muestran un comportamiento de cluster glass, mientras que para las mayores concentraciones de Mn (76 \%at. y 83 \%at.) el sistema responde como un aglomerado de partículas acopladas antiferromagnéticamente. 


\subsection{Sistema $\mathrm{FeMnCu}$}

En lo que respecta al sistema ternario, éste ha sido poco investigado, enfocándose la mayoría de los trabajos encontrados al estudio en la región de altas concentraciones de Cu. A continuación se presentan algunos de estos reportes.

\subsubsection{Trabajos previos}

Miettinen $^{52}$ realizó cálculos para describir la termodinámica de las fases de alta temperatura del sistema $\mathrm{FeMnCu}$, basándose en los datos aportados por los diagramas de fases de las aleaciones binarias. La descripción sólo es válida en la región con contenidos de Mn menores al 40 \%at., y se consideraron para el estudio tres fases: líquida, $f c c$ y bcc. El modelo está basado en la minimización de la energía libre de Gibbs, donde se incluye un término que contempla el ordenamiento magnético. Las curvas de las fases simuladas fueron comparadas con información experimental, encontrándose en la mayoría de los casos un buen acuerdo.

Con respecto al estudio estructural, Restrepo y otros ${ }^{53}$ estudiaron el sistema $\left(\mathrm{Fe}_{50} \mathrm{Mn}_{50}\right)_{30} \mathrm{Cu}_{70}$ en función del tiempo de molienda. Para la fabricación de las aleaciones se empleó un molino planetario, variando el tiempo de molienda desde 15 min hasta $120 \mathrm{~h}$. Los difractogramas de rayos $\mathrm{X}$ mostraron que la única fase presente es la $f c c$, con un parámetro de red de $3.615 \AA$ para 15 min incrementándose hasta $3.690 \AA$ para el mayor de los tiempos. Asimismo, observaron una disminución del tamaño de grano con el tiempo de molienda. Además argumentaron que la reproducción del difractograma mejora cuando en el ajuste se incluye un término de anisotropía de forma, sugiriendo ésto la presencia de deformaciones en la partícula producto del método de fabricación. Otro estudio fue realizado por Stewart et. al. ${ }^{54}$ en muestras preparadas por molienda mecánica. Prepararon dos concentraciones, $\left(\mathrm{Fe}_{50} \mathrm{Mn}_{50}\right)_{10} \mathrm{Cu}_{90}$ y $\left(\mathrm{Fe}_{50} \mathrm{Mn}_{50}\right)_{20} \mathrm{Cu}_{80}$, que se caracterizaron por DRX, EM y medidas de susceptibilidad magnética. Para cada una de las concentraciones se realizaron dos preparaciones, en la primera se agregó $\mathrm{Cu}$ metálico a una aleación previa de FeMn, mientras que en la segunda se obtuvo la aleación partiendo de los tres metales. Los resultados estructurales de todas las muestras revelaron que la única fase presente es la $f c c$, con un parámetro de red levemente superior al del Cu metálico. Alocén y colaboradores ${ }^{55}$ exponen resultados sobre una muestra de composición $\left(\mathrm{Fe}_{50} \mathrm{Mn}_{50}\right)_{20} \mathrm{Cu}_{80}$ preparada por molienda mecánica en un molino de alta energía, en función del tiempo de molienda. Los resultados de DRX 
muestran que, para tiempos de molienda menores a 120 h, existen rastros de los elementos Fe y Cu, no pudiéndose saber si las líneas del Mn están presentes debido a la coincidencia de su reflexión más intensa con la línea (111) del $\mathrm{Cu}{ }^{56}$ Para tiempos mayores, la única fase que observaron fue la fcc. Ésta presentó un parámetro de red levemente mayor al del Cu puro en coincidencia con lo observado por Stewart y otros. ${ }^{54}$ Los tamaños de cristalita determinados, empleando la fórmula de Scherrer, muestran una disminución desde $70 \mathrm{~nm}$ hasta $30 \mathrm{~nm}$. Por último, el trabajo de França et. al. ${ }^{57}$ es de los pocos que se enfoca en la región de bajos contenidos de $\mathrm{Mn}$ y Cu. En él se presentan resultados estructurales, hiperfinos y magnéticos en muestras de $\mathrm{Fe}_{86} \mathrm{Mn}_{14-\mathrm{y}} \mathrm{Cu}_{\mathrm{y}}$ ( $\mathrm{y}=1,3$ y 5) preparadas en un horno de arco, con un posterior tratamiento térmico a $1273 \mathrm{~K}$. Los difractogramas indicaron que en todas las concentraciones la única fase presente es la bcc, con un parámetro de red promedio de $2.88 \AA$, mayor que el encontrado para el Fe puro en la bibliografía. ${ }^{58}$ Los autores atribuyeron este incremento a una transferencia de carga entre los átomos de $\mathrm{Mn}$ y $\mathrm{Cu}$ en la solución sólida $\mathrm{FeMnCu}$, que induce un aumento en la ionicidad del sistema, conduciendo a un aumento de la distancia media entre los átomos.

En lo que respecta al estudio de las propiedades hiperfinas de la aleación $\left(\mathrm{Fe}_{50} \mathrm{Mn}_{50}\right)_{30} \mathrm{Cu}_{70}$ Restrepo y otros ${ }^{53}$ observaron que después de 21 h de molienda, los espectros Mössbauer llegan a un estado estacionario. En estas condiciones se propone un ajuste para los espectros medidos a 77 K considerando dos dobletes asimétricos con valores del desdoblamiento cuadrupolar $(\Delta \mathrm{Q})$ de 0.41 $\mathrm{mm} / \mathrm{s}$ y $0.45 \mathrm{~mm} / \mathrm{s}$ y corrimientos isoméricos de $0.43 \mathrm{~mm} / \mathrm{s}$ y $0.23 \mathrm{~mm} / \mathrm{s}$, respectivamente. Con el aumento del tiempo de molienda el doblete de $\Delta \mathrm{Q}=0.41 \mathrm{~mm} / \mathrm{s}$ sufre un brusco cambio en el valor de su corrimiento isomérico siendo su nuevo valor $0.12 \mathrm{~mm} / \mathrm{s}$. Ésto fue atribuido a una transición de un estado de bajo espín a uno de alto espín. Este hecho es reforzado por la variación del corrimiento isomérico con el parámetro de red, similar al encontrado en aleaciones de FeNi. ${ }^{59}$ Stewart y colaboradores ${ }^{54}$ encontraron para las muestras $\left(\mathrm{Fe}_{50} \mathrm{Mn}_{50}\right)_{10} \mathrm{Cu}_{90}$ y $\left(\mathrm{Fe}_{50} \mathrm{Mn}_{50}\right)_{20}$ que los espectros Mössbauer medidos a temperatura ambiente pueden ser reproducidos empleando dos dobletes, en algunos casos siendo necesario sumarle una línea simple al ajuste. Los valores del corrimiento isomérico para los dobletes varían entre $0.13 \mathrm{~mm} / \mathrm{s}$ y $0.28 \mathrm{~mm} / \mathrm{s}$, por ésto dichas interacciones fueron asignadas a la presencia de regiones de $\mathrm{FeCu}$ ricas en $\mathrm{Cu}$ y a la propia aleación ternaria, mientras que la línea simple, con un corrimiento isomérico de $0.22 \mathrm{~mm} / \mathrm{s}$, fue atribuida a átomos de Fe aislados en la matriz de $\mathrm{Cu}$. Medidas realizadas a baja temperatura mostraron que los espectros Mössbauer adquieren un carácter magnético a temperaturas que concuerdan con la presencia de un máximo en las curvas de susceptibilidad. França y otros ${ }^{57}$ encontraron, debido al rango composicional de su trabajo $\mathrm{Fe}_{86} \mathrm{Mn}_{14-\mathrm{y}} \mathrm{Cu}_{\mathrm{y}}(\mathrm{y}=1,3 \mathrm{y}$ 5), espectros Mössbauer con interacciones magnéticas resueltas. Éstos son reproducidos con una distribución de campos magnéticos hiperfinos con un valor del campo medio de $31.5 \mathrm{~T}$ y observándose varios máximos en la distribución de probabilidad, los que reflejan los distintos entornos atómicos del Fe. 
El estudio magnético del sistema mostró, en el trabajo de Restrepo et. al. ${ }^{53}$ un comportamiento tipo vidrio de espín, con temperaturas de congelamiento entre $55 \mathrm{~K}$ y $66 \mathrm{~K}$. En base a estos valores atribuyeron este comportamiento a la segregación de CuMn, con un 87 \%at. de $\mathrm{Cu}$. Por su parte, en el trabajo de Stewart ${ }^{54}$ encontraron que las curvas de susceptibilidad presentaron un máximo con valores de la sensitividad con la frecuencia entre los esperados para un superparamagneto y un vidrio de espín. ${ }^{60}$ Este último comportamiento es atribuido, basándose en las temperaturas del máximo en la susceptibilidad, a la presencia de aglomerados de $\mathrm{Cu}_{83} \mathrm{Mn}_{17}$. Alocén et. al..$^{55}$ encontraron, basándose en medidas ZFC-FC, la presencia de dos fases magnéticas. La primera de ellas se encontró a altas temperaturas y fue asociada a una fase tipo cluster-glass, formada principalmente por átomos de Fe con acoplamientos a primeros y segundos vecinos. La segunda de las fases, de baja temperatura, fue asociada a un comportamiento de vidrio de espín producto de átomos aislados de Fe y Mn acoplados entre sí por los electrones de conducción del $\mathrm{Cu}$. Ciclos de histéresis medidos a temperatura ambiente muestran una dependencia lineal de la magnetización con el campo magnético aplicado, no observándose saturación aun para campos de 5 T. Las medidas magnéticas realizadas por França y $\operatorname{otros}^{57}$ mostraron que la susceptibilidad responde principalmente al comportamiento magnético de la matriz de Fe. Se destaca una región entre 450 K y $700 \mathrm{~K}$ donde fue evidente la oxidación de las muestras, observándose, además, cómo las distintas concentraciones afectan levemente a la temperatura de Curie del sistema. Finalmente, ciclos de histéresis medidos a temperatura ambiente presentan un comportamiento característico de un material ferromagnético blando, con una remanencia y coercitividad despreciable. La magnetización de saturación disminuye a medida que el contenido de Mn aumenta, lo cual sugirió que el mismo se acopla antiferromagnéticamente con el Fe. 


\subsection{Referencias}

\footnotetext{
${ }^{1}$ H Otsuka, H Yamada, T Maruyama, H Tanahashi, S. Matsuda y M. Murakami; ISIJ International 30, 8 (1990), pág.674.
}

${ }^{2}$ K. Otsuka, C.M. Wayman (editores), en “Shape Memory Materials”, Cambridge University Press, (1999).

${ }^{3}$ A. Sato, E. Chishima, K. Soma y T. Mori; Acta Metall. 30, 6 (1982), pág. 1177.

${ }^{4}$ R.W. Crossa, Young K. Kim, J.O. Oti y S.E. Russek; Appl. Phys. Lett. 69, 25 (1996), pág. 3935.

${ }^{5}$ J. P. King, J. N. Chapman, J.C.S. Kools y M.F. Gillies; J. Phys. D: Appl. Phys. 32, (1999), pág. 1087.

${ }^{6}$ F.R. de Boer, R. Boom, W.C. M. Mattens, A.R. Miedema y A.K. Niessen, en "Cohesión and Structure”, vol.1, ed. F. R. de Boer and D. G. Pettifor (North-Holland, Amsterdam, 219, (1989).

7 D.R. Lide (editor), en "CRC Handbook of Chemistry and Physics", Version 2009, http://www.hbcpnetbase.com, CRC Press, Boca Raton, FL, (2009), pág 12-119.

${ }^{8}$ T.B. Massalski, en “Binary Alloy Phase Diagrams” $2^{\text {da }}$ Edición, H. Okamoto, P. Subramanian y L. Kacprzak (editores), Vol. 2, pág. 1724.

${ }^{9}$ H. Umebayashi y Y. Ishikawa; Phys. Soc. Jpn. 21, (1966), pág. 1281.

${ }^{10}$ C. Paduani, E. Galvão da Silva, G.A. Perez-Alcazar y M. McElfresh; J. App. Phys. 70, 12 (1991), pág. 7524.

${ }^{11}$ C. Paduani, E. Galvão da Silva y G.A. Perez-Alcazar; Hyp. Int. 73, 3-4 (1992), pág. 233.

12 J. Martínez, S.M. Cotes, A.F. Cabrera, J. Desimoni y A. Fernández Guillermet; Mat. Sci. Eng. A 408, 1-2 (2005), pág. 26.

${ }^{13}$ Y. Endoh y Y. Ishikawa; J. Phys. Soc. Jpn. 30, (1971), pág. 1614.

${ }^{14}$ M. Uhrmacher, A. Kulinska, Yu.V. Baldokhin. V.V. Tcherdyntsev, S.D. Kaloshkin, A. Maddalena y G. Principi; Hyperfine Interactions 136/137, 3-8 (2001), pág. 327.

${ }^{15}$ H. Fujimori; J. Phys. Soc. Jpn. 21, (1966), pág. 1860.

${ }^{16}$ J. Martínez, S.M. Cotes y J. Desimoni; J. Alloys Comp. 479, 1-2 (2009), pág. 204.

${ }^{17}$ J. Martínez, S.M. Cotes y J. Desimoni; Phys. Status Solidi B 246, 6 (2009), pág. 1366.

${ }^{18}$ V.V. Cherdyntsev, S.D. Kaloshkin, I.A. Tomilin, E.V. Shelekhov y Yu. V. Baldokhin; Phys. Met. Metall. 94, 4 (2002), pág. 391.

${ }^{19}$ M. Uhrmacher, A. Kulinska, Yu. V. Baldokhin, V. V. Tcherdyntsev, S. D. Kaloshkin, A. Maddalena y G. Principi; Intermetallics 10, 6 (2002), pág. 571.

${ }^{20}$ P. Chubing y D. Daosheng; J. Appl. Phys. 76, (1994), pág. 2986.

${ }^{21}$ F.R. de Boer, R. Boom, W.C.M. Mattens, A.R. Miedema y A.K. Niessen, en "Cohesion in Metals, Transition Metal alloys”, North-Holland, Ámsterdam (1989), pág. 240. 
${ }^{22}$ Y. Kimura y S. Takaki; ISIJ International 37, 3 (1997), pág. 290.

${ }^{23}$ K. Sumiyama y Y. Nakamura; J. Magn. Magn. Mater. 35 (1983), pág. 219.

24 P.H. Shingu, en "Mechanical alloying, Proceedings of Internacional Symposium on Metastable, Mechanically Alloyed and Nanocrystaline Materials”, (ISMANAM), Kyoto, Japan, (1991).

${ }^{25}$ L.J. Swartzendruber, en “Binary Alloy Phase Diagrams” $2^{\text {da }}$ Ediciónn, H. Okamoto, P. Subramanian y L. Kacprzak (editores), Vol. 2, pág. 1408.

${ }^{26}$ C.L. Chien, S.H. Liou, D. Kofalt, Wu Yu, T. Egami, Thomas J. Watson y T.R. McGuire; Phys. Rev. B 33, 5 (1985), pág. 3247.

${ }^{27}$ P.H. Shingu, K.N. Ishihara, K. Uenishi, J. Kuyama, B. Huan y S. Nasu, en "Solid State Powder Processing”, A.H. Clauer y J.J. de Barbadillo (Editores), The Minerals, Metals and Materials Society, (1990), pág. 21.

${ }^{28}$ K. Uenishi, K.F. Kobayashi, K.N. Ishihara y P.H. Shingu; Mater. Sci. Eng. A 134, (1991), pág.1342.

${ }^{29}$ A.R. Denton y N.W. Ashcroft; Phys. Rev. A 43, 6 (1990), pág. 3161.

${ }^{30}$ J. Eckert, J.C. Holzer, C.E. Krill III y W.L. Jonson; J. Mater. Res 7, 8 (1992), pág. 1980.

${ }^{31}$ J. Eckert, J.C. Holzer y W.L. Jonson; J. App. Phys. 73, 1 (1993), pág. 131.

${ }^{32}$ A.R. Yavari, P.J. Desré y T. Benameur; Phys. Rev. Lett. 68, 14 (1992), pág. 2235.

${ }^{33}$ E. Ma, M. Atzmon y F.E. Pinkerton; J. App. Phys. 74, 2 (1993), pág. 955.

${ }^{34}$ P. Crespo, A. Hernando, R. Yavari, O. Drbohlav, A. García Escorial, J.M. Barandiarán y I. Oru'e; Phys. Rev. B 48, 10 (1993), pág. 7134.

${ }^{35}$ J.Y. Huang, A.Q. He y Y.K. Wu; Nanostruct. Materials 4, 1 (1994), pág. 1.

${ }^{36}$ J.Z. Jiang, U. Gonser y R. Bormann; Mat. Sci. and Eng. A 242, (1998), pág. 268.

${ }^{37}$ P.P. Macri, P. Rose, R. Frattini, S. Enzo, G. Principi, W.X. Hu y N. Cowlam; J. Appl Phys 76, 7 (1994), pág. 4061.

${ }^{38}$ T. Ambrose, A. Gavrin y C.L. Chien; J. Magn. Magn. Mater. 14, 1-2 (1993), pág. L15.

${ }^{39}$ J. Ding, M. Eilon, R. Street, T. St. Pierre, P. Smith y P.G. McCormick; J. Magn. Magn. Mater. 140144, (1995), pág. 471.

${ }^{40}$ M. Eilon, J. Ding y R. Street; J. Phys.: Condens. Matter 7, 25 (1995), pág. 4921.

${ }^{41}$ S.S. Saxena, J. Tang, Y.S. Lee y C.J. O'Connor; J. App. Phys. 76, 10 (1994), pág. 6820.

${ }^{42}$ Y. Ueda, S. Ikeda, S. Moriwaki y M. Matsuda; Jpn. J. App. Phys. 35, part 2, 8b (1996), pág. L1059.

${ }^{43}$ F.R. de Boer, R. Boom, W.C.M. Mattens, A.R. Miedema y A.K. Niessen, en "Cohesion in Metals, Transition Metal alloys”, North-Holland, Ámsterdam, (1989).

${ }^{44}$ F.A. Shunk, en "Binary Alloy Phase Diagrams” $2^{\text {da }}$ Edición, H. Okamoto, P. Subramanian y L. Kacprzak (editores), Vol. 2, pág. 1435.

${ }^{45}$ D. Meneghetti y S.S. Sidhu; Phys. Rev. 105, 1 (1955), pág. 130. 
${ }^{46}$ B. Window; J. Phys. C: Solid State Phys. 3, (1970), pág. 922.

${ }^{47}$ S.F. Edwards y P.W. Anderson; J. Phys. F: Metal Phys. 5, (1975), pág. 965.

${ }^{48}$ C.A.M. Mulder, A.J. van Duyneveldt y J.A. Mydosh; Phys. Rev. B 23, 3 (1980), pág. 1384.

${ }^{49}$ S. Shtrikman y E.P. Wohlfarth; Phys. Letters A, 85, 8-9 (1981), pág. 467.

${ }^{50}$ A. Banerjee y A.K. Majumdar; Phys. Rev. B 46, 14 (1992), pág. 8958.

${ }^{51}$ David Jiles, en “Introduction to Magnetism and Magnetic Materials”, ${ }^{\text {da }}$ Edición, CRC Press, (1998).

52 J. Miettinen; Computer Coupling of Phase Diagrams and Thermochemistry 27, (2003), pág. 141.

53 J. Restrepo, J.M. Grenèche y J.M. González; Physica B: Condensed Matter 354, 1-4 (2004), pág. 174.

${ }^{54}$ S.J. Stewart, J.F. Marco, P. Crespo, J.J. Romero, A. Martínez, A. Hernando, F.J. Palomares y J.M. González; J. Nanosci. Nanotechnol. 7, 2 (2007), pág. 610.

${ }^{55}$ M.C. Alocén, P. Crespo, A. Hernando y J.M. González; J. Non-Cryst. Solids 287, 1-3 (2001), pág. 268.

${ }^{56}$ JCPDS- International Center for Diffraction Data, PCPDFWIN v.2.1, tarjeta No 85-1326.

${ }^{57}$ F. França, C. Paduani, J.C. Krause, J.D. Ardisson, M.I. Yoshida y J. Schaf; Physica B: Condensed Matter 387, 1-2 (2007), pág. 292.

58 JCPDS- International Center for Diffraction Data, PCPDFWIN v.2.1, tarjeta No 06-0696.

${ }^{59}$ K. Lagarec y D.G. Rancourt; Phys. Rev. B 62, 2 (2000), pág. 978.

60 J.A. Mydosh, Tabla 3.1 en "Spin glasses: An Experimental Introduction”, ed. Taylor \& Francis, London, (1993). 


\section{Capítulo 3. Algunas propiedades magnéticas de sistemas nanoestructurados}

\section{Introducción}

Se puede considerar que un sistema magnético nanoscópico está formado por partículas o aglomerados (clusters) magnéticos, cuyo tamaño puede ir desde unos pocos nanometros hasta decenas de ellos. Se pueden encontrar sistemas con una distribución de tamaños nanométricos en sólidos (llamados sólidos granulares) o en medios líquidos (llamados ferrofluidos). En el caso de sólidos granulares, la matriz en la que se hallan inmersas las nanopartículas puede ser aislante o conductora, cristalina o amorfa, y puede contener más de una fase de diferentes materiales. De este modo, las propiedades físicas de los sistemas de nanopartículas pueden ser fabricadas o modificadas de acuerdo a un interés científico o tecnológico específico. ${ }^{1,2}$ Se puede destacar la utilización de nanopartículas para la producción de imanes más poderosos o materiales magnéticos blandos con menor pérdida de energía, así como también en la producción de microsensores magnéticos y una infinidad de otras aplicaciones tales como en diagnóstico médico, catálisis, líquidos magnéticos para uso en medicamentos, pigmentos en pinturas y cerámicas. ${ }^{3,4}$ Fundamentalmente, el gran impulso en el estudio de estos sistemas que ha surgido en los últimos años se debe a las enormes perspectivas de su uso en sistemas computacionales, sea para la lectura o grabación, o en el propio almacenamiento de información utilizando la dirección y sentido de los momentos magnéticos de las nanopartículas. ${ }^{5}$ Se pueden obtener partículas sólidas ultrafinas a través de diversos métodos, tales como reducción química, ${ }^{6}$ deposición por vapor, sputtering, ${ }^{7}$ melt-spinning, ${ }^{8}$ electrodeposición ${ }^{9}$ o aleado mecánico, ${ }^{10}$ siendo esta última técnica la empleada para preparar las muestras estudiadas en esta Tesis. Las dimensiones reducidas de las partículas, junto con las nanoestructuras formadas presentan una rica variedad de propiedades físicas que, además de su relevancia tecnológica, forman un conjunto único para estudiar diversos problemas interesantes en física del estado sólido. Ejemplos son el superparamagnetismo ${ }^{11,12}$ la cinética de nucleación y crecimiento de granos, ${ }^{13,14}$ y el comportamiento tipo vidrio de espín..$^{15,16}$ 


\subsection{Sistemas de partículas no interactuantes}

Se considerarán las propiedades magnéticas de un conjunto de partículas no interactuantes con una distribución amplia de tamaños y con una distribución aleatoria de ejes de fácil magnetización. Tal sistema puede ser estudiado dentro de la teoría superparamagnética. Este nombre fue introducido por Bean y Livingston ${ }^{17}$ por la analogía que presentan los mismos con sistemas paramagnéticos. La primera suposición de la teoría superparamagnética es considerar que los momentos magnéticos atómicos en el interior de una partícula se mueven coherentemente, o sea que el momento magnético total puede ser representado por un único vector clásico de magnitud $\boldsymbol{\mu}$ $=\boldsymbol{\mu}_{a t} N$, donde $\boldsymbol{\mu}_{a t}$ es el momento magnético atómico y $N$ es el número de átomos magnéticos que conforman dicha partícula. En el caso más simple, la dirección del momento magnético es determinada por la anisotropía uniaxial (de origen magnetocristalina, de forma, o magnetoelástica) y por el campo magnético externo. Es decir, una partícula muy pequeña tendrá una dirección preferencial llamada eje de fácil magnetización, que es, como se verá más adelante, la dirección hacia la cual preferentemente se orienta el vector momento magnético.

\subsubsection{Tiempos de relajación y bloqueo del sistema}

La relajación temporal de estas partículas consistentes de un monodominio magnético puede ser descripta con una ley de tipo Arrhenius: ${ }^{18}$

$$
M(t)=M_{0} \exp [-t / \tau]
$$

donde $M o$ es la magnetización inicial y $\tau$ es el tiempo característico de relajación del sistema. Este tiempo característico es función de la barrera de energía y de la temperatura como lo expresa la siguiente relación:

$$
\tau=\tau_{0} \exp \left[E_{B} / k_{B} T\right]
$$

donde $\tau_{0}$ está asociado a la frecuencia de tentativas de saltos del momento magnético de la partícula entre sentidos opuestos del eje de fácil magnetización. Su valor puede ser determinado experimentalmente y también puede ser predicho a través de modelos teóricos. ${ }^{19,20}$ Los valores aceptados hoy en día para $\tau_{0}$ se hallan comprendidos entre $10^{-9}$ y $10^{-10}$ segundos. $E_{B}$ es la barrera 
de energía que separa a los dos estados de equilibrio y $k_{B}$ es la constante de Boltzmann. La barrera de energía viene dada por el producto entre el volumen de la partícula $(V)$ y la constante de anisotropía $K_{A}$. En los sistemas superparamagnéticos el comportamiento magnético observado depende del valor del tiempo típico de medición de la técnica experimental $\left(\tau_{m}\right)$ respecto al tiempo de relajación propio del sistema $(\tau)$, asociado a la barrera de energía. Si $\tau_{m}>>\tau$, la relajación del sistema ocurre más rápido que el tiempo durante el cual se está realizando la medida, dejando que el sistema llegue al equilibrio termodinámico. Lo que se observa en este caso es que el conjunto de partículas se comporta de modo análogo a un sistema paramagnético. Si por el contrario $\tau_{m}<<\tau$, la relajación del sistema resulta muy lenta y se observan propiedades cuasiestáticas, como en los sistemas magnéticamente ordenados. Cuando el sistema se encuentra en este régimen se dice que se encuentra bloqueado. La temperatura que separa estos regímenes se denomina temperatura de bloqueo $\left(T_{B}\right)$ y depende del tiempo característico de medición $\tau_{m}$. La temperatura de bloqueo, que se define como aquella en la que $\tau=\tau_{m}$, está asociada a la barrera de energía y por ello se incrementa con el aumento de tamaño de la partícula. Por el contrario, para un dado tamaño, $T_{B}$ disminuye con el incremento del tiempo característico de medición. A continuación en la Tabla 3.1 se muestran algunos tiempos característicos de medida para distintas técnicas experimentales.

\begin{tabular}{|c|c|c|c|c|}
\cline { 2 - 5 } \multicolumn{1}{c|}{} & \multicolumn{4}{c|}{ Instrumento de medida } \\
\cline { 2 - 5 } \multicolumn{1}{c|}{} & Esp. Mössbauer & Susceptómetro AC & VSM & Magnetómetro (SQUID) \\
\hline$\tau_{m}(\mathrm{~s})$ & $10^{-8}$ & $10^{-4}$ a 1 & 10 & 10 a 100 \\
\hline
\end{tabular}

Tabla 3.1: Tiempos característicos de medida de diversas técnicas experimentales.

Para una partícula de simetría uniaxial (ver figura 3.1a) la anisotropía magnética puede ser escrita como:

$$
E_{\theta}=E_{B} \operatorname{sen}^{2}(\theta)
$$

donde $\theta$ es el ángulo entre la magnetización y el eje de fácil magnetización. La energía magnética tiene dos mínimos simétricos $A$ y $B$, como se muestra con la línea sólida en la figura 3.1b, que corresponden a $0^{\circ}$ y $180^{\circ}$. Aplicando un campo magnético $H$ en la dirección del eje $z$, la energía magnética se reescribe como:

$$
E_{\theta}=E_{B} \operatorname{sen}^{2}(\theta)-\mu \cdot \mathrm{H}
$$

${ }^{\mathrm{i}}$ El tiempo de medida en susceptibilidad AC es inversamente proporcional a la frecuencia del campo de medida. 
Como se ve en la figura 3.1b, si el campo magnético externo $(H)$ es nulo, la barrera de energía entre los dos estados de equilibrio tiene un valor igual a $K_{A} V$. A medida que aumenta el valor de $H$, el pozo de la izquierda comienza a hacerse más notorio, mientras que el pozo de la derecha tiende a desaparecer, disminuyendo el valor de la barrera de energía a $\Delta \mathrm{E}$.

(a)

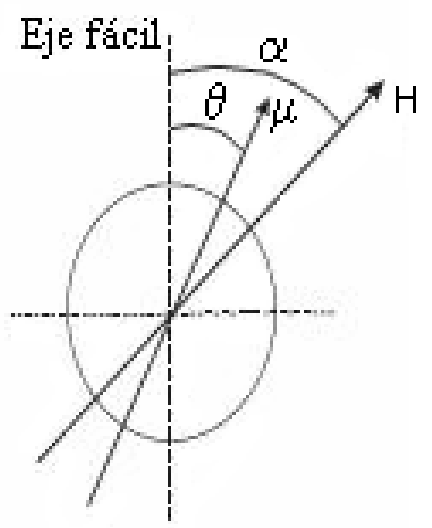

(b)

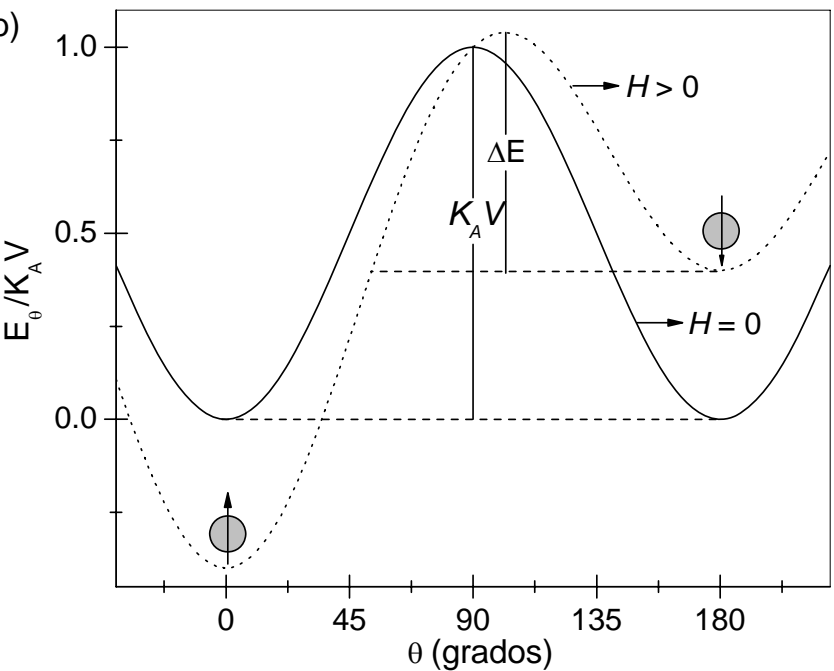

Figura 3.1 a): Esquema de la partícula considerada con los ejes de magnetización y dirección del campo magnético aplicado.

Figura 3.1 b): Dependencia angular de la barrera de energía sin campo aplicado (línea sólida) y en presencia de un campo magnético externo (línea punteada).

Se puede definir un volumen crítico $\left(V_{c}\right)$ a una cierta temperatura $T_{0}$ como el volumen de las partículas para el cual el sistema está en el límite de ser detectado como magnéticamente ordenado o como paramagnético. Análogamente a la forma en la que se determinó la temperatura de bloqueo imponiendo que $\tau=\tau_{m}$, se puede determinar el valor del $V_{c}$. Si en la expresión 3.2 se reemplaza $\tau$ por $\tau_{m}$, el volumen en esta expresión pasará a ser el volumen crítico para las partículas del sistema:

$$
\tau=\tau_{0} \exp \left[\frac{K_{A} V}{k_{B} T_{0}}\right] \Rightarrow \tau_{m}=\tau_{0} \exp \left[\frac{K_{A} V_{c}}{k_{B} T_{0}}\right]
$$

y $V_{c}$ puede ser escrito como: 


$$
V_{c}=\frac{k_{B} T_{0}}{K_{A}} \ln \left(\frac{\tau_{m}}{\tau_{0}}\right)
$$

donde se observa que el volumen crítico a una dada temperatura, dependerá del tiempo de medida de la técnica empleada. A continuación, en la Tabla 3.2, se dan las expresiones para el volumen crítico para distintas técnicas experimentales.

\begin{tabular}{|c|c|c|c|c|}
\cline { 2 - 5 } \multicolumn{1}{c|}{} & \multicolumn{4}{c|}{ Instrumento de medida } \\
\cline { 2 - 5 } \multicolumn{1}{c|}{} & Esp. Mössbauer & Susceptómetro AC ${ }^{\mathrm{ii}}$ & VSM & Magnetómetro (SQUID) \\
\hline$V_{c}$ & $\frac{2 k_{B} T_{0}}{K_{A}}$ & $\frac{12 k_{B} T_{0}}{K_{A}}$ a $\frac{20 k_{B} T_{0}}{K_{A}}$ & $\frac{23 k_{B} T_{0}}{K_{A}}$ & $\frac{25 k_{B} T_{0}}{K_{A}}$ \\
\hline
\end{tabular}

Tabla 3.2: Volúmenes críticos determinados para las diversas técnicas experimentales.

De la tabla anterior se puede notar que, partículas de un determinado volumen pueden ser detectadas como magnéticas o no magnéticas, dependiendo de la ventana temporal de la técnica que se esté usando para estudiarlas. Por otra parte, de aquí también se puede ver que el volumen crítico de una partícula para que presente un comportamiento superparamagnético es directamente proporcional a la temperatura, o sea, cuanto mayor sea la temperatura mayor será el tamaño crítico de las partículas, y todas aquellas que tengan un tamaño menor o igual al crítico estarán en régimen superparamagnético. Si se tiene una distribución de tamaños, al elevar la temperatura cada vez más partículas comenzaran a comportarse como superparamagnéticas. Un análisis similar se puede hacer de la dependencia de la temperatura de bloqueo con el volumen de las partículas. De la Ec. 3.7 se ve que la temperatura de bloqueo es directamente proporcional tanto al volumen de las partículas como a la constante de anisotropía, o sea, partículas más grandes van a tener temperaturas de bloqueo más altas.

$$
T_{B}=\frac{K_{A} V}{k_{B}} \ln \left(\frac{\tau_{0}}{\tau_{m}}\right)
$$

En la figura 3.2 se muestra la dependencia del radio crítico, $R_{c}=\left(\frac{3}{4 \pi} V_{c}\right)^{1 / 3}$, y del tiempo de relajación con la temperatura para partículas esféricas de Fe de 50 nm de radio.

ii El valor de la izquierda y el de la derecha corresponde al tiempo de medida de $10^{-4}$ y 1 s respectivamente. 


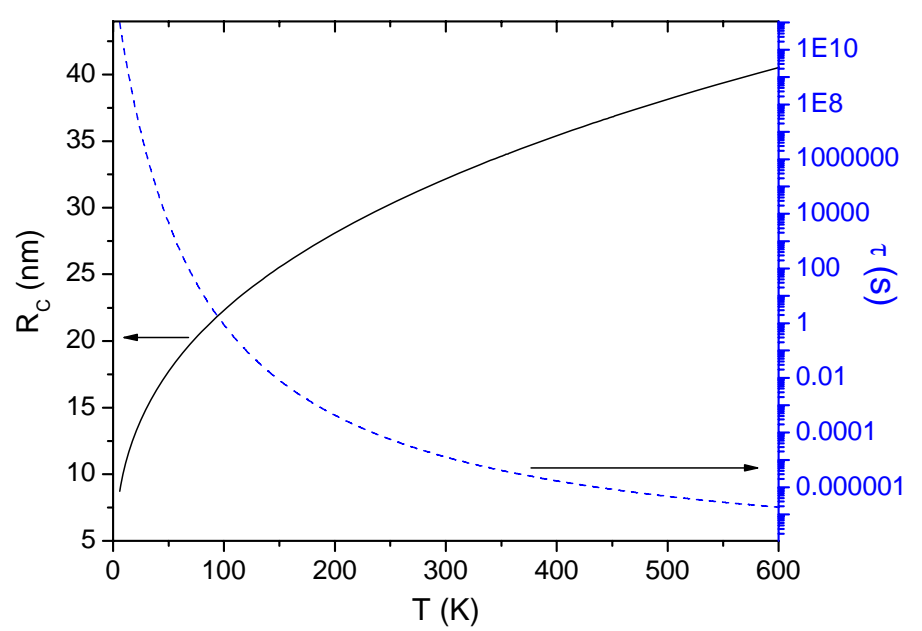

Figura 3.2: Variación del radio crítico y del tiempo de relajación en función de la temperatura para una barrera de energía fija.

Existe claramente una transición entre el comportamiento bloqueado y el comportamiento superparamagnético, donde los momentos magnéticos exploran en su conjunto distintas configuraciones posibles. Para altas temperaturas, o partículas extremadamente pequeñas, el tiempo característico de relajación térmica resulta ser mucho menor que cualquier tiempo característico utilizado en los equipos de medición y, por lo tanto, el momento magnético puede "saltar" de un pozo de potencial al otro varias veces durante la medición; así el sistema se detectará como superparamagnético. Por otro lado, cuando la temperatura es suficientemente baja, la escala temporal $\tau$ se torna muy grande, mucho mayor que cualquier tiempo de observación, en esta circunstancia, los momentos magnéticos permanecen "congelados” sin poder explorar nuevos estados, la activación térmica no es suficiente para superar la barrera de energía, y por lo tanto en estas condiciones el sistema estará bloqueado. Para tener una idea de los números que se manejan en este tipo de experimentos se puede dar un ejemplo. Una partícula esférica de Fe con $60 \AA$ de diámetro tiene un tiempo característico de relajación de apenas un décimo de segundo. Por lo tanto, si dichas partículas son exploradas en un magnetómetro DC, se obtiene como resultado que el conjunto de partículas es no magnético. Mientras que si se aumenta en un $50 \%$ su diámetro, el valor de $\tau$ crece 10 órdenes de magnitud $\left(3.2 \times 10^{9} \mathrm{~s}\right)$, lo cual significa que el momento magnético es tan estable que demoraría en promedio 100 años para sufrir una inversión. No obstante el momento magnético intenta traspasar la barrera con una frecuencia del orden de diez mil millones de veces por segundo. Esos números varían de material en material y dependen de la forma de las partículas y de su nivel de tensión mecánica, aunque en general la variación del tiempo de relajación con la dimensión de las partículas es muy brusca. 


\subsection{Conjuntos de partículas, fórmula de Langevin}

Se considerará un conjunto de partículas formadas por un monodominio, cada una con un momento magnético $\mu$ y anisotropía despreciable. A pesar de que el magnetismo es un fenómeno puramente cuántico, se puede considerar al momento magnético $\mu$ como un vector clásico, pues se estará suponiendo que los momentos magnéticos de cada átomo, dentro de cada partícula, están acoplados ferromagnéticamente. De este modo, todos estos momentos magnéticos estarán fuertemente correlacionados, aún durante una rotación del momento total. Por lo tanto, el tratamiento estadístico de este sistema puede seguir la misma formulación clásica del paramagnetismo, sólo que con un valor del momento magnético mucho mayor que el de los átomos. Se considerará que el sistema se encuentra a una temperatura $T$ lo suficientemente alta, en presencia de un campo magnético $H$, y que ha alcanzando el equilibrio termodinámico. A esa temperatura todas las partículas se encontrarán en el estado superparamagnético. Se tiene así una distribución de Boltzmann de los momentos $\mu$ con relación al campo $H$, de modo análogo al caso del paramagnetismo clásico. Cada momento magnético tiene una cierta energía potencial $E_{p}$ dada por:

$$
E_{P}=-\vec{\mu} \cdot \vec{H}=-\mu H \operatorname{Cos}(\theta) .
$$

El número de momentos $d n$ entre $\theta$ y $\theta+d \theta$ es proporcional a $d A$, multiplicado por el factor de Boltzmann:

$$
d n=K d A \exp \left(\frac{-E_{p}}{k_{B} T}\right)=2 \pi K \exp \left(\frac{\mu H \operatorname{Cos}(\theta)}{k_{B} T}\right) \operatorname{Sen}(\theta) d \theta
$$

donde $K$ es un factor de proporcionalidad determinado por la condición:

$$
\int_{0}^{n} d n=n
$$

Multiplicando el número de momentos magnéticos $d n$ por la contribución $\mu \cos (\theta)$ de cada momento, e integrando sobre el número total de momentos, se obtiene la magnetización total $M$ : 


$$
M=n \mu\left[\operatorname{Cotgh}\left(\frac{\mu H}{k_{B} T}\right)-\frac{k_{B} T}{\mu H}\right]
$$

donde $n \mu$ es el máximo valor posible del momento que el material podría tener y corresponde al alineamiento perfecto de todos los momentos magnéticos con el campo. Esa magnetización corresponde a la magnetización de saturación $M_{\mathrm{SL}}$ :

$$
\frac{M}{M_{S L}}=\operatorname{Cotgh}\left(\frac{\mu H}{k_{B} T}\right)-\frac{k_{B} T}{\mu H}=L\left(\frac{\mu H}{k_{B} T}\right)
$$

donde $L$ es la función de Langevin. En la figura 3.3 se representa $M / M_{S L}$ vs. $H / T$ para distintos valores del parámetro $\alpha=\mu / k_{B}$.

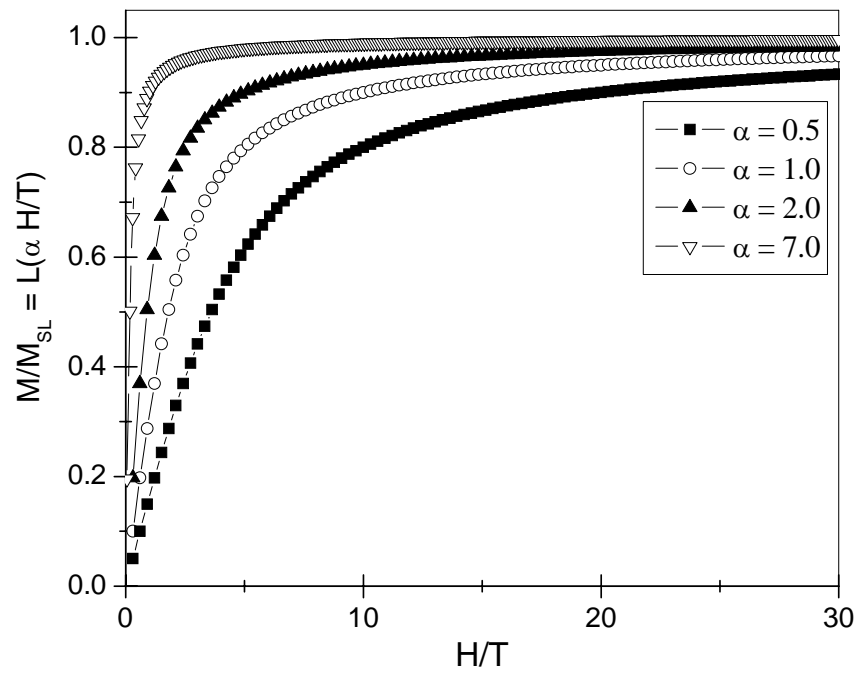

Figura 3.3: Comportamiento de la función de Langevin en función de H/T para distintos valores del parámetro $\alpha$.

De la Ec. 3.12 se ve que es relativamente simple analizar si un sistema es superparamagnético o no. Basta realizar medidas de magnetización vs. campo magnético a diversas temperaturas. Posteriormente si se realiza el grafico de $M / M_{S}$ vs. $H / T$ se espera que todas las curvas converjan a una única curva universal (curva de Langevin). En los sistemas reales, por lo general, no se tiene un buen colapso de todas las curvas debido a que en éstos existe una distribución de tamaños, con lo cual se podrían tener a una dada temperatura momentos magnéticos que estén bloqueados y otros que no. Otro motivo por el cual la convergencia puede no observarse es la existencia de una distribución aleatoria de ejes de anisotropía o la presencia de interacciones entre partículas. Los 
sistemas de partículas con los que se trabaja comúnmente presentan una distribución de tamaños de partículas. Si $f(\mu)$ es la función distribución de tamaños de partícula, la magnetización macroscópica estará dada por: $:^{21,22}$

$$
M(H, T)=\int_{0}^{\infty} \mu L\left(\frac{\mu H}{k_{B} T}\right) f(\mu) d \mu
$$

Para aplicar la Ec. 3.13 a datos experimentales se debe considerar una función de distribución apropiada. Generalmente, observaciones a través de microscopía electrónica indican que la distribución de tamaños en sistemas granulares sigue una función distribución del tipo lognormal: $:^{22}$

$$
f(\mu)=\int_{0}^{\infty} \frac{N}{\sqrt{2 \pi}} \exp \left[-\frac{\operatorname{Ln}^{2}\left(\mu / \mu_{0}\right)}{2 \sigma^{2}}\right] d \mu,
$$

donde $N$ es el número de partículas del sistema, $\sigma$ es la desviación cuadrática media de la distribución y $\mu_{0}$ está relacionado con el valor del momento magnético medio $(\langle\mu\rangle)$ por $\langle\mu\rangle=\mu_{0}$ exp $\left(\sigma^{2} / 2\right)$. De este modo, los parámetros $\mu_{0}$ y $\sigma$ pueden ser determinados a través de un ajuste apropiado de la curva de magnetización experimental. Además de la distribución de tamaños, si se considera que las partículas poseen forma esférica, es posible obtener la densidad de partículas y la distancia media entre ellas. ${ }^{22}$ Existen otras medidas magnéticas que pueden ser utilizadas para estudiar sistemas superparamagnéticos. Las medidas magnéticas macroscópicas en función de la temperatura son muy importantes y aportan considerable información fundamental al estudio de partículas finas. ${ }^{23}$ En este tipo de medidas se puede mencionar las mediciones de magnetización enfriando sin campo magnético aplicado (Zero Field Cooling, ZFC) y enfriando con campo magnético aplicado (Field Cooling, FC). Del análisis de la derivada de la diferencia entre las curvas $Z F C$ y $F C$ se puede estimar la distribución de las temperaturas de bloqueo de las partículas bajo estudio, pudiendo inferir de dicho gráfico la distribución de tamaños de las partículas. ${ }^{24}$ Otra técnica muy utilizada es la medida de la susceptibilidad AC, utilizando diversas frecuencias para el campo de excitación. Al cambiar la frecuencia, se modifica el número de partículas que logran acompañar esta variación y, por lo tanto, la respuesta magnética de este tipo de sistema se modifica sensiblemente. 


\subsection{Sistema interactuantes}

En los sistemas nanoscópicos reales se tiene que considerar la contribución de muchas partículas con diferentes tamaños y formas, aún sin considerar las interacciones entre las mismas, el estudio se torna complicado. Es interesante notar que la complejidad de los problemas hace que las soluciones exactas sólo sean posibles en casos límites, tales como $T=0 \mathrm{~K}$, para partículas completamente bloqueadas (modelo de Stoner-Wohlfarth ${ }^{25}$ ), o para $T>>T_{B}$, o sea, un sistema totalmente superparamagnético. ${ }^{26} \mathrm{El}$ análisis de estos sistemas se torna todavía más complejo si se consideran las interacciones entre las partículas magnéticas presentes en diferentes sistemas. ${ }^{9,27,28}$ La mayoría de los resultados de simulaciones concuerdan en que las interacciones magnetoestáticas producen un aumento en la $T_{B}$, en acuerdo con resultados experimentales, ${ }^{29}$ sin embargo el efecto de las interacciones sigue siendo un tema de debate hoy en día.

\subsubsection{Interacciones magnéticas}

A continuación se dará una breve descripción de algunas interacciones magnéticas que se pueden presentar entre los momentos magnéticos. Por simplicidad se hablará siempre de interacciones entre pares de espines atómicos, siendo también válida la descripción para momentos magnéticos que provengan de un comportamiento colectivo de espines como ocurre en sistemas donde existen aglomerados magnéticos.

\section{Interacción dipolar}

Un mecanismo por el cual los espines pueden interactuar es a través de la interacción dipolar. Aunque este tipo de acoplamiento es débil, está siempre presente y el Hamiltoniano que describe esta interacción entre dos átomos tiene la forma:

$$
H_{i j}^{d i p}=\frac{1}{r_{i j}^{3}}\left[\vec{\mu}_{i} \cdot \vec{\mu}_{j}-3\left(\vec{\mu}_{i} \cdot \breve{r}_{i j}\right)\left(\vec{\mu}_{j} \cdot \breve{r}_{i j}\right)\right]
$$

donde $\mu_{\mathrm{i}} \mathrm{y} \mu_{\mathrm{j}}$ son los momentos magnéticos de los respectivos átomos y $\mathrm{r}_{\mathrm{ij}}$ la distancia entre ellos. En este tipo de interacción, además de la dependencia con la inversa de la distancia al cubo, existe también una anisotropía intrínseca a la interacción dipolar, la cual puede orientar a los espines en forma ferromagnética o antiferromagnéticamente. Por ejemplo, si los espines se encuentran 
orientados a lo largo de $r_{i j}$, ellos estarán acoplados ferromagnéticamente, sin embargo, si los espines están orientados perpendicularmente a $r_{i j}$ el acoplamiento será antiferromagnético. Por lo tanto, a diferencia de otro tipo de interacciones, la interacción dipolar introduce una dependencia angular en el acoplamiento entre espines.

\section{Intercambio directo}

El intercambio directo involucra un solapamiento de las funciones de onda electrónicas de dos átomos y las consecuencias que ésto trae sobre la paridad de la parte espacial y de la parte de espín de dicha función de onda. Por el principio de exclusión de Pauli, dos electrones que posean sus espines paralelos se mantendrán lo más apartados el uno del otro y viceversa. Las interacciones magnéticas de intercambio entre dos espines $S_{i}$ y $S_{j}$ son usualmente representadas por un Hamiltoniano de spin:

$$
H_{i j}=-J_{i j} \vec{S}_{i} \cdot \vec{S}_{j}
$$

donde $J_{i j}$ es la integral de intercambio. Se define la energía de intercambio como la diferencia de energía entre las configuraciones de espines paralelos y antiparalelos. Debido a que las funciones de onda de los electrones $d$ o $f$ decrecen exponencialmente con la distancia al núcleo, el valor de la integral de intercambio $J_{i j}$ será muy pequeña en estos casos.

\section{Interacción RKKY}

En sistemas constituidos por átomos magnéticos inmersos en una matriz conductora, los electrones de conducción de esta última llevan a la existencia de una interacción fuerte de largo

alcance. Ésta es conocida como interacción RKKY debido a que Ruderman, Kittel, Kasuya y Yosida fueron los primeros en dar cuenta de ella. ${ }^{30}$ Colocar átomos magnéticos en una matriz no magnética de un metal conductor produce un amortiguamiento oscilatorio en la susceptibilidad de los electrones de conducción, y con ello un acoplamiento de los espines $S_{i}$ y $S_{j}$ entre dos de los átomos magnéticos, el cual puede ser descripto por la siguiente función:

$$
J(r)=6 \pi Z J^{2} N\left(E_{F}\right)\left[\frac{\operatorname{sen}\left(2 k_{F} r\right)}{\left(2 k_{F} r\right)^{4}}-\frac{\cos \left(2 k_{F} r\right)}{\left(2 k_{F} r\right)^{3}}\right]
$$


aquí $Z$ es el número de electrones de conducción por átomo, $J$ la constante de intercambio, $N\left(E_{F}\right)$ la densidad electrónica del nivel de Fermi, $k_{F}$ el momento de Fermi y $r$ la distancia entre dos átomos magnéticos. La expresión anterior a largas distancias se puede reducir a:

$$
J(r)=\frac{J_{0} \cos \left(2 k_{F} r+\phi\right)}{\left(2 k_{F} r\right)^{3}}
$$

donde el factor $\phi$ se incluye como una fase adicional para tener en cuenta la diferencia de carga entre los átomos magnéticos y los conductores. El comportamiento oscilatorio de $J(r)$ es el que hace interesante a esta interacción, ya que la misma acoplará, ferromagnética o antiferromagnéticamente, a átomos dependiendo de la distancia que exista entre ellos. También se puede notar que la dependencia radial $r^{-3}$ sólo permitirá un acoplamiento entre sitios cercanos. Si se combinan estas propiedades con un desorden estructural (posibilidad de separaciones distintas entre átomos magnéticos), se podrá generar una distribución aleatoria de acoplamientos, con distintas intensidades y sentidos, los cuales podrán llevar al sistema a una eventual frustración magnética. 


\subsection{Vidrios de espín}

En los sistemas de vidrios de espín hay interacciones competitivas entre espines, o conjuntos de ellos. Para comenzar, un vidrio de espín es un sistema en el cual no existe orden de largo alcance, pero, sin embargo, puede tener un orden magnético. Los ingredientes principales de un vidrio de espín son desorden e interacciones magnéticas competitivas que llevan al sistema a un estado denominado frustrado. El desorden puede ser tanto estructural como atómico y/o químico. El desorden estructural está ligado a un sistema que posee diferentes distancias entre los átomos magnéticos, el desorden atómico está relacionado con la presencia de distintos entornos atómicos en diferentes regiones del sistema, mientras que el desorden químico está asociado a la presencia de diferentes tipos de enlaces entre los átomos. El segundo de los ingredientes, la frustración magnética, está relacionada con la existencia de interacciones competitivas que conducen a que algunos de los espines del sistema no puedan optar por una determinada orientación. Esta frustración de los espines impide que el sistema encuentre una condición de equilibrio estable, que minimice simultáneamente cada término del Hamiltoniano, conduciendo a un complicado paisaje de energías con múltiples estados fundamentales. Como consecuencia de ésto los vidrios de espín exhiben propiedades dinámicas y termodinámicas muy complejas.

Los vidrios de espín canónicos son los compuestos por alguna impureza magnética (Mn, Fe, $\mathrm{Gd}, \mathrm{Eu}$, etc) en una matriz no magnética conductora ( $\mathrm{Cu}, \mathrm{Au}$, etc). En este tipo de vidrios de espín, la interacción magnética RKKY es la responsable de crear la frustración de los espines. Posteriormente se encontró que otros tipos de compuestos como el $\mathrm{Eu}_{\mathrm{x}} \mathrm{Sr}_{1-\mathrm{x}} \mathrm{S}$ (semiconductor), el $\mathrm{La}_{1-\mathrm{x}} \mathrm{Gd}_{\mathrm{x}} \mathrm{Al}$ (metal), el $\mathrm{GdAl}_{2}$ y el $\mathrm{YFe}_{2}$ (intermetálicos amorfos) presentaban características similares. ${ }^{31,32}$ También se pudo encontrar sistemas en los cuales interacciones como la dipolar o la de intercambio son responsables del comportamiento de vidrio de espín.

Los vidrios de espín presentan respuestas particulares ante determinadas técnicas experimentales, siendo en muchos casos éstas fundamentales para poder discernir qué tipo de comportamiento magnético está mostrando el sistema estudiado.

En vidrios de espín canónicos, la medida de la susceptibilidad AC en función de la temperatura muestra una cúspide bien definida a la temperatura de congelamiento $\left(T_{\max }\right)$, mientras que la susceptibilidad fuera de fase ( $\chi$ ’”) posee una abrupta caída a dicha temperatura. Sin embargo, pueden ser encontradas características similares en sistemas de partículas superparamagnéticas con una distribución de tamaños de partícula, por lo que se tendrá que ampliar el tipo de caracterización para determinar si se está en presencia de un material tipo vidrio de espín. Una de las principales diferencias entre un superparamagneto y un vidrio de espín es que en el 
primero existe un bloqueo progresivo de sus partículas debido a la distribución de tamaños, mientras que el segundo proceso se podría pensar como más cercano a una transición de fase. Esta diferencia produce que en un superparamagneto la temperatura del máximo de la curva de susceptibilidad dependa fuertemente de la ventana temporal del experimento, mientras que en un vidrio de espín esta dependencia debería ser casi nula. En ambos casos $T_{\max }$ se desplaza hacia temperaturas mayores a medida que la ventana temporal disminuye, siendo una forma de cuantificar esta variación calcular el corrimiento del máximo por década de frecuencia: ${ }^{33}$

$$
p=\frac{\Delta T}{\bar{T} \Delta \log (v)},
$$

donde $\Delta \mathrm{T}$ es la diferencia entre la temperatura del máximo de la susceptibilidad medida a dos frecuencias distintas y $\Delta \log (v)$ es la diferencia entre los logaritmos de dichas frecuencias. Dado que los valores de $p$ son del orden de algunas décimas para un superparamagneto y algunas milésimas para un vidrio de espín, este es un buen criterio para diferenciar entre estos tipos de comportamientos.

Un primer análisis para interpretar el comportamiento del máximo de las curvas de susceptibilidad en un vidrio de espín, puede ser hecho a partir de la ley de Arrhenius, ${ }^{18}$ la cual fue previamente introducida para explicar procesos térmicamente activados como el superparamagnetismo (Ec. 3.2). Los datos obtenidos en la bibliografía ${ }^{34}$ muestran que para sistemas donde la variación de $T_{\max }$ con la frecuencia es débil, la ley de Arrhenius predice valores de los parámetros $E_{B}$ y $\tau_{0} \sin$ significado físico. Esto es debido a que en los vidrios de espín no existe una simple barrera de energía, la cual puede ser sobrepasada con la temperatura.

Un segundo método de análisis es a partir de la ley de Vogel-Fulcher. ${ }^{35}$ Esta ley empírica fue empleada en sus comienzos para describir el comportamiento de líquidos sobreenfriados y posteriormente se aplicó a los sistemas de partículas magnéticamente interactuantes, estando su expresión dada por:

$$
\tau=\tau_{0} \exp \left[\frac{E_{B}}{k_{B}\left(T_{\max }-T_{0}\right)}\right]
$$

donde $T_{0}$ es el nuevo parámetro introducido que denotará la temperatura de la transición, siendo $T_{\max }$ sólo una manifestación dinámica de la misma. Con esta expresión es posible reproducir el comportamiento de $T_{\max }$ con la frecuencia en sistemas de vidrios de espín, obteniéndose valores de los parámetros más realistas. El verdadero significado físico de $T_{0}$ está en discusión, pero es hoy en 
día aceptado como una medida del grado de interacción entre los átomos o clusters de un vidrio de espín.

Finalmente, una tercera aproximación puede ser obtenida a partir de la teoría del escaleo dinámico en torno a un punto crítico. ${ }^{36}$ Esta teoría relaciona la longitud de correlación entre los espines de la red $(\xi)$ con el tiempo de relajación de los mismos $(\tau)$ a través de $\tau \sim \xi^{\mathrm{z}}$, donde $z$ es el exponente crítico. Por otra parte, $\xi$ diverge con la temperatura según $\xi \sim\left[T_{\mathrm{c}} /\left(T_{\max }-T_{\mathrm{c}}\right)\right]^{v}$, donde $v$ es otro exponente crítico. Usando estas dos expresiones se puede obtener que la dependencia de $T_{\max }$ con el tiempo de medida está dada por:

$$
\tau=\tau_{0}\left[\frac{T_{\max }-T_{c}}{T_{c}}\right]^{-z v}
$$

donde los valores de $\tau_{0}, T_{c}$ y $z \vee$ pueden ser obtenidos de un ajuste de datos experimentales. Los valores encontrados para vidrios de espín ${ }^{37,38}$ están entre $10^{-12}$ y $10^{-15}$ segundos para $\tau_{0}$, mientras que $z v$ puede variar entre 4 y 12, siendo el valor esperado para una transición de fase convencional igual a 2.

Por otra parte, si se aplica un campo externo DC en la medida de susceptibilidad AC el cambio observado es mucho más significativo. Para campos relativamente bajos ( 10 Oe) el máximo de la curva se desplazará hacia temperaturas menores, disminuirá en intensidad y se tornará más redondeado. Estos cambios afectan sólo a la región cercana al máximo, siendo casi imperceptible el efecto en las otras partes de la curva. Almeida y Thouless ${ }^{39}$ han propuesto que en los sistemas de vidrios de espín la relación entre $T_{\max } \mathrm{y}$ un campo externo DC tendrá una dependencia del tipo $T_{\max } \propto H^{2 / 3}$.

Para estudiar la dependencia de la susceptibilidad AC con un campo aplicado $\left(H_{D C}\right)$ se define la susceptibilidad no lineal, la cual es un desarrollo en serie de la magnetización en términos de dicho campo:

$$
M \approx M_{0}+\chi H_{D C}-M_{3} H_{D C}^{3}+\ldots
$$

El término independiente es introducido, por lo general, para contemplar la magnetización remanente en las bobinas del instrumento de medida, mientras que el tercer término es el que tendrá en cuenta los efectos no lineales presentes en el sistema. Se espera que en las cercanías de la temperatura $T_{\max }$ el coeficiente de este término diverja, mostrando de esta forma la cercanía de una transición de fase. Varios autores ${ }^{40,41}$ coinciden en que este tipo de análisis es el más adecuado para determinar si existe o no una transición de fase termodinámica en un vidrio de espín; sin embargo, 
los resultados experimentales muestran comportamientos intermedios, lo cual continúa haciendo difícil la interpretación.

Las medidas de magnetización con $(F C)$ y $\sin (Z F C)$ campo aplicado durante el enfriado son otra herramienta útil para identificar un sistema de tipo vidrio de espín. La figura 3.4 muestra las curvas típicas de $Z F C$ - $F C$ para un vidrio de espín canónico. Como se puede apreciar, ambas curvas coinciden en la región de altas temperaturas, cuando se alcanza la temperatura del máximo de las curvas, y a medida que se continúa bajando la temperatura, la magnetización de la curva $F C$ permanece sin cambios, mientras que la magnetización $Z F C$ comienza a disminuir. Por lo tanto, si se realiza una medida $F C$ bajando y luego subiendo la temperatura a campo constante se observará que la curva recorre el mismo camino, dando como resultado que la curva $F C$ es un proceso reversible. Por otra parte, a bajas temperaturas $\left(T<T_{\max }\right)$ deteniéndose sobre un punto de la curva de magnetización $Z F C$ se observará que éste posee una lenta deriva que hace aumentar el valor de su magnetización. Si se espera un tiempo suficientemente largo el valor de la magnetización $Z F C$ tenderá a coincidir con el valor de la curva $F C$, esto es $\mathrm{M}_{\mathrm{ZFC}}(t \rightarrow \infty) \approx \mathrm{M}_{\mathrm{FC}}$. Por lo tanto, la curva $Z F C$ es dependiente de la velocidad con la que varía la temperatura en el proceso de medida, pudiendo dar como resultado una irreversibilidad en la curva ZFC. A diferencia de la susceptibilidad AC, donde la medida es reversible, cuando se comparan curvas de magnetización $Z F C$ entre sí, es muy importante tomar las precauciones necesarias para que los protocolos de medida sean iguales a fin de no introducir errores provenientes de la irreversibilidad del proceso.

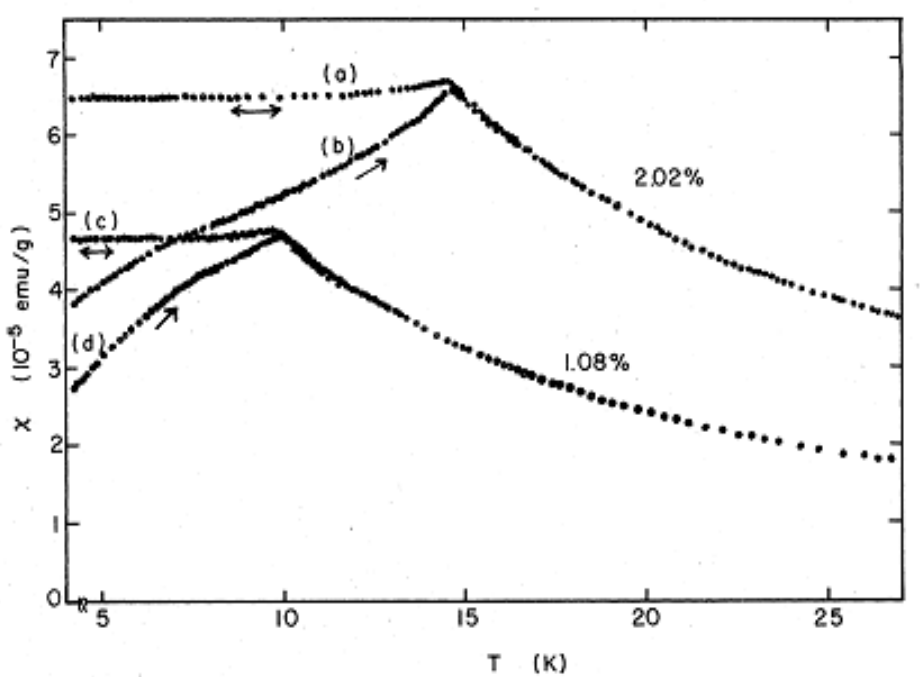

Figura 3.4: Curvas de magnetización ZFC y FC para el vidrio de espín canónico CuMn con los porcentajes de $\mathrm{Mn}$ indicados un la figura. 


\subsubsection{Clusters glass}

A medida que la concentración de los átomos magnéticos aumenta, existe una gran probabilidad de que dos átomos magnéticos se encuentren como primeros o segundos vecinos entre sí. Ya que la función de onda de los electrones $3 d$ en un metal de transición tiene una extensión finita ellos también pueden transportar una polarización RKKY. Consecuentemente, puede existir un tipo de interacción RKKY de corto alcance que puede acoplar átomos magnéticos vecinos, pudiendo ser el acoplamiento ferromagnético o antiferromagnético, dependiendo del átomo magnético y de la posición entre vecinos. Como consecuencia de esta interacción y de fluctuaciones composicionales en la solución sólida, pueden formarse aglomerados magnéticos los cuales pueden tener un orden ferromagnético o antiferromagnético. Cuando el comportamiento magnético del sistema está dominado por la presencia de estos aglomerados magnéticos el término mictomagneto o cluster glass es usado en la bibliografía, en la actualidad muchos autores llaman a este comportamiento como superspinglass. La presencia de estas grandes entidades magnéticas simplifica la detección del proceso de congelamiento ya que, en estos sistemas tanto la magnetización como la susceptibilidad muestran valores mucho más elevados que las de un vidrio de espín. La presencia de los aglomerados también hace que el sistema posea una gran irreversibilidad cuando se estudian las curvas de histéresis y la presencia de un desplazamiento lateral de los ciclos cuando la medida se realiza posteriormente a haber enfriado el sistema en presencia de un campo magnético (exchange bias). 


\subsection{Referencias}

\footnotetext{
${ }^{1}$ G. Storma, S.O. Belliota, T. Daemenb y D.D. Lasic; Adv. Drug Del. Rev. 17, 1 (1995), pág. 31.

${ }^{2}$ C. Barrera, A.P. Herrera, Y. Zayas y C. Rinaldi; J. Magn. Magn. Mater. 321, (2009), pág. 1397.

${ }^{3}$ G.C. Hadjipanayis y G.A. Prinz (editores), en "Science and Technology of Nanostructured Magnetic Materials”, Vol. 259 de NATO Advanced Study Institute, Series B: Physics, Plenum Press, New York, (1991).
}

4 J.L. Dormann y D. Fiorani (editores), en "Magnetic Properties of Fine Particles", North-Holland, Amsterdam, (1992).

${ }^{5}$ M.N. Baibich, en “Magnetism, Magnetic materials and their applications", F. Leccabue y V. Sagrado (editores), World Scientific, Singapur, (1996), pág. 69.

${ }^{6}$ K.C. Huang y S.H. Ehrman; Langmuir, 23, 3 (2007), pág. 1419.

${ }^{7}$ T. Ichitsubo, M. Koujina, M. Kawashima y M. Hirao; Jpn. J. Appl. Phys. 42, (2003), pág. 2858.

${ }^{8}$ S.P.H. Marashi, A. Abedi, S. Kaviani, S. H. Aboutalebi, M. Rainforth y H.A. Davies; J. Phys. D: Appl. Phys. 42, (2009), pág. 115410.

${ }^{9}$ L. Da-ling, D. Kazunari y T. Ken-ichi; Langmuir, 18, 8 (2002), pág. 3226.2

${ }^{10}$ E.P. Yelsukov y G.A. Dorofeev; Hyp. Int. 164, 1-4 (2005), pág. 51.

${ }^{11}$ S. Mørup y E. Tronc; Phys. Rev. Lett. 72, (1994), pág. 3278.

${ }^{12}$ C.L. Chien; J. Appl. Phys. 69, (1991), pág. 5267.

${ }^{13}$ A.Hütten y G. Thomas; Ultramicroscopy 52, (1993), pág. 581.

${ }^{14}$ A. López, F.J. Lázaro, R. von Helmolt, J.L. García-Palacios, J. Wecker y H. Cerva; J. Magn. Magn. Mater. 187, (1998), pág. 221.

15 J.L. Dormann, R. Cherkaoui, L. Spanu, M. Noguès, E. Tronc y J.P. Jolivet; J. Magn. Magn. Mater. 187, (1998), pág. L139.

16 J.R. Childress y C.L. Chien; Phys. Rev. B 43, (1991), pág. 8089.

${ }^{17}$ C.P. Bean y J.D. Livingston; J. Appl. Phys. 30, (1959), pág. 120.

${ }^{18}$ J.L. Dormann, L. Bessais y D. Fiorani; J. Phys. C: Solid State Phys. 21, (1998), pág. 2015.

${ }^{19}$ B.D. Cullity, en “Introduction to Magnetic Materials”, Addison-Wesley Publishing Co., Reading, (1972).

${ }^{20}$ P. Allia, M. Coisson, M. Knobel, P. Tiberto y F. Vinai; Phys. Rev. B 60, (1999), pág. 12207.

${ }^{21}$ M.G.M. Miranda, G.J. Bracho Rodríguez, A.B. Antunes, M.N. Baibich, E.F. Ferrari, F.C.S. da Silva y M. Knobel; J. Magn. Magn. Mater. 185, (1998), pág. 331. 
${ }^{22}$ E.F. Ferrari, F.C.S. Silva y M. Knobel; Phys. Rev. B 56, (1997), pág. 6086.

${ }^{23}$ L.M. Socolovsky y F.H. Sánchez; Materials Characterization 50, (2003), pág. 123.

${ }^{24}$ J.L. Dormann, D. Fiorani y E. Tronc; Adv. Chem. Phys. 98, (1997), pág. 283.

${ }^{25}$ E.C. Stoner y E.P. Wohlfarth; Phil. Trans. Roy. Soc. A 240, (1948), pág. 599.

${ }^{26}$ D.A. Dimitrov y G.M. Wysin; Phys. Rev. B 54, (1996), pág. 9237.

${ }^{27}$ P. Allia, M. Knobel, P. Tiberto y F. Vinai; Phys. Rev. B 52, (1995), pág. 15398.

${ }^{28}$ J.L. Dormann, F. D'Orazio, F. Lucari, E. Tronc, P. Prené, J. P. Jolivet, D. Fiorani, R. Cherkaoui y M. Noguès; Phys. Rev. B 53, (1996), pág. 14291.

${ }^{29}$ M. El-Hilo, R.W. Chantrell y K. O’Grady; J. Appl. Phys. 84, (1998), pág. 5114.

${ }^{30}$ J. Crangle, en “Solid State Magnetism”, $1^{\text {ra }}$ ed., Springer, (1991), pág. 143.

${ }^{31}$ H. Maletta y W. Felsch; Phys. Rev. B 20, 3 (1979), pág. 1245.

${ }^{32}$ G.F. Zhou y H. Bakker; Phys. Rev. Lett. 73, 2 (1994), pág. 344.

33 J.A. Mydosh, en "Spin glasses: an experimental introduction”, Ed. Taylor \& Francis, London-Washinton, DC (1993), pág. 66.

${ }^{34}$ J.A. De Toro, M.A. López de la Torre, M.A. Arranz, J.M. Riveiro y J.L. Martínez; J. Appl. Phys. 87, 9 (2000), pág. 6534.

${ }^{35}$ S. Shtrikman y E.P. Wohlfarth; Phys. Letters A, 85, 8-9 (1981), pág. 467.

${ }^{36}$ C. Djurberg, P. Svedlindh, P. Nordblad, M.F. Hansen, F. Bødker y S. Mørup; Phys. Rev. Lett. 79, 25 (1997), pág. 5154.

${ }^{37}$ P. Jönsson, M.F. Hansen, P. Svedlindh y P. Nordblad; J. Magn. Magn. Mater. 226-230, part 2 (2001), pág. 1315.

${ }^{38}$ W. Kleemann, O. Petracic, Ch. Binek, G.N. Kakazei, Y.G. Pogorelov, J.B. Sousa, S. Cardoso y P.P. Freitas; Phys. Rev. B 63, 13 (2001), pág. 134423.

39 J.R.L. de Almeida y D.J. Thouless; J. Phys. A 11, (1978), pág. 983.

${ }^{40}$ O. Cador, F. Grasset, H. Hanedab y J. Etourneau; J. Magn. Magn. Mater. 268, (2004), pág. 232.

${ }^{41}$ J.A. De Toro, M.A. López de la Torre, J.M. Riveiro, R. Sáez Puche, A. Gómez-Herrero y L.C. OteroDíaz; Phys. Rev. B 60, 18 (1999), pág. 12918. 


\section{Capítulo 4. Técnicas experimentales y análisis de datos}

En este capítulo se presenta el modo de preparación de las muestras y su posterior montaje, junto a una descripción de las condiciones experimentales utilizadas para la caracterización de las mismas. Los fundamentos detrás de cada técnica, pueden ser consultados en la bibliografía sugerida. ${ }^{1-8}$

\subsection{Técnica de aleado mecánico y preparación de las muestras}

\subsubsection{Aleado Mecánico}

La técnica de aleado mecánico es una herramienta útil para la preparación de aleaciones estables o metaestables. Es un proceso de alta energía, lo cual la hace fundamental para el mezclado de los elementos a escala atómica; por esto, el aleado mecánico se considera un método de síntesis sumamente apropiado para toda clase de sistemas tanto metálicos como iónicos. Las transformaciones de estado sólido inducidas mecánicamente dependen fuertemente de la energía mecánica entregada, de las fuerzas termodinámicas involucradas y de la cinética del proceso, quienes determinarán, junto con las características del elemento o compuesto, el producto final obtenido. ${ }^{9}$

Existen diversos dispositivos mecánicos que consisten, generalmente, de dos o más contenedores dentro de los cuales se colocan esferas de un material de menor dureza que el del contenedor y mayor que la del material a alear. La transferencia de energía y cantidad de movimiento se produce mediante los impactos de las esferas sobre el polvo que queda atrapado 
entre las mismas o entre una esfera y la pared del contenedor. La energía absorbida por el material puede producir transformaciones de fase, desplazamientos atómicos, fractura de los granos de material, agregación de granos, generación de interfases y creación de nanoestructuras.

\subsubsection{Molino vibratorio horizontal}

El molino vibratorio horizontal fue la herramienta utilizada en este trabajo para la preparación de las muestras. Se utilizó un molino de marca Retsch modelo MM 400, éste consta de dos brazos horizontales en los cuales se pueden colocar cilindros de $1.5 \mathrm{~cm}^{3}$ a $10 \mathrm{~cm}^{3}$ de volumen con esferas de diferentes tamaños en su interior. La frecuencia de oscilación de los brazos del molino se puede variar en un rango de $1 \mathrm{a} 33 \mathrm{~Hz}$. En este tipo de molino, las esferas no están sometidas a ninguna fuerza en la dirección del movimiento, por lo que su velocidad antes de impactar nuevamente contra la pared del contenedor será la misma que adquirió al despegarse de la pared opuesta. La transferencia de energía al material ocurre mediante estos impactos. Para registrar la frecuencia de molienda durante todo el proceso se acopló al molino un circuito electrónico conectado a una computadora. A continuación en la figura 4.1 se muestra una fotografía de los elementos empleados para realizar la molienda.
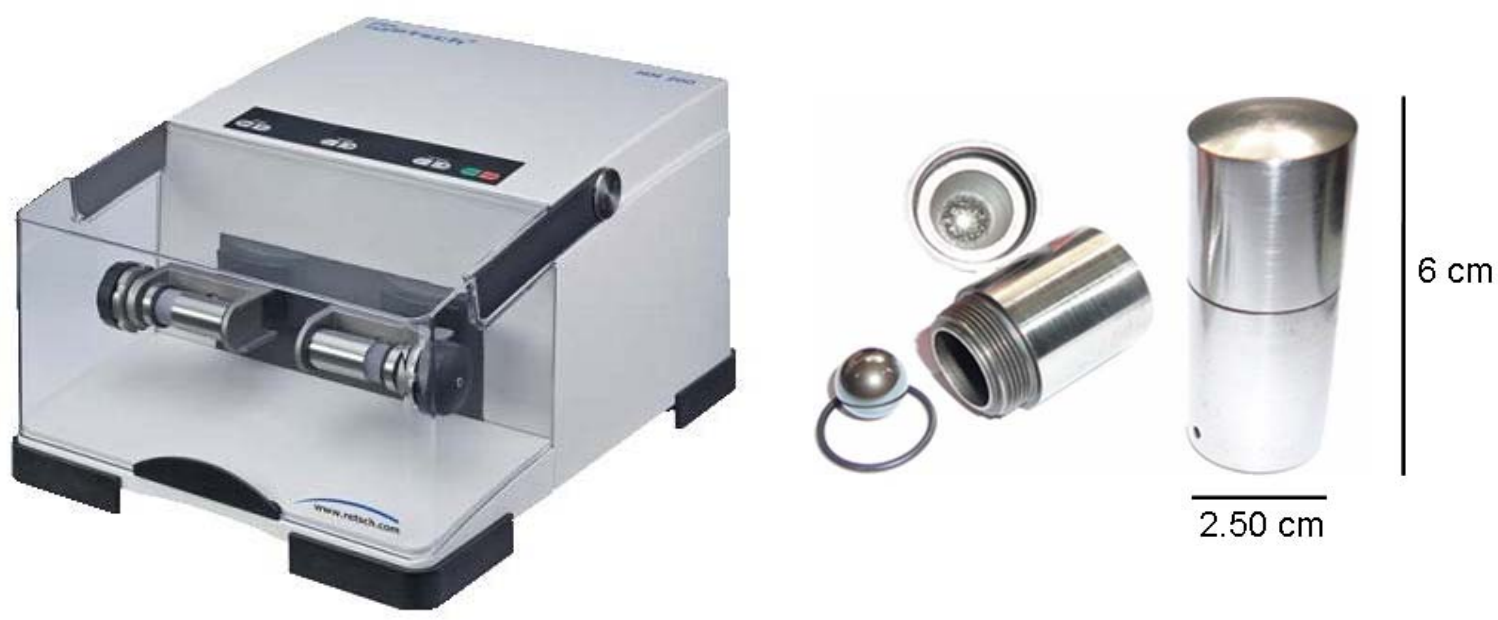

Figura 4.1: Fotografía del molino vibratorio horizontal, similar al empleado para la preparación de las muestras de esta tesis (izq). Cilindros y esferas usados en el proceso de molienda (der). 


\subsubsection{Preparación de las muestras}

Para la preparación de las muestras se partió de los elementos puros Fe (99.98 \% de pureza), Mn (99.98\% de pureza) y Cu (99.99\% de pureza). El Fe de partida consistió en trozos de $1 \mathrm{~mm}^{3}$ aproximadamente y el $\mathrm{Cu}$ en pequeñas esferas. El Mn estaba fraccionado en láminas de $1 \mathrm{~cm}^{2}$ aproximadamente, por lo que antes de preparar la aleación se las transformó en polvo. Para ésto se molió durante 15 minutos las láminas, previamente limpiadas con una solución de nital al 5\% para eliminar cualquier óxido superficial que se pudiera haber formado. La figura 4.2 muestra las fotografías de los metales empleados.
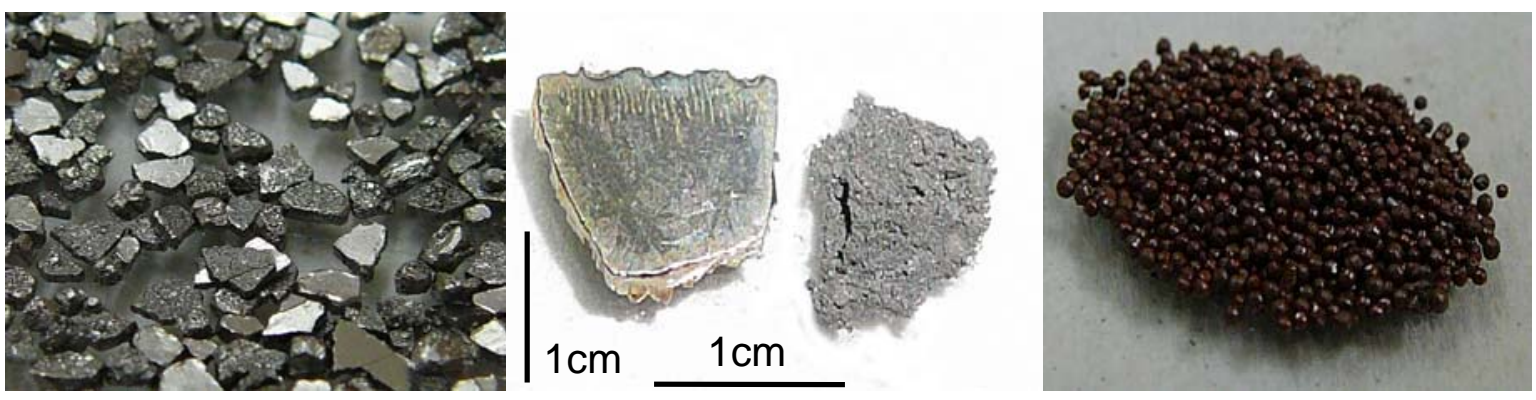

Figura 4.2: Metales empleados para la preparación de las muestras: Hierro (izq.), Manganeso (centro) y Cobre (der).

Las muestras de FeMnCu fueron preparadas pesando los metales en una balanza analítica con una precisión de $0.1 \mathrm{mg}$, siendo el peso total 300 mg por preparación. Una vez pesados en las proporciones adecuadas, se procedió a colocar los metales junto con una esfera de acero de $9 \mathrm{~mm}$ de diámetro en un cilindro de acero tipo $\mathrm{K}^{\mathrm{i}}$ de $10 \mathrm{~cm}^{3}$, con una relación masa de esfera a masa de muestra de 20/1. El contenedor se cerró en atmósfera de Ar. Este último proceso se realizó colocando el cilindro abierto con los metales y la bola en su interior dentro en un globo, luego se efectuaron sucesivos lavados con Ar, de modo que los metales queden expuestos a una atmósfera inerte para evitar la oxidación de los mismos.

Una vez cerrados los cilindros se colocaron en el molino (hasta dos por molienda), el cual funcionó a una frecuencia de $30 \mathrm{~Hz}$, con intervalos de descanso de 15 minutos cada 45 minutos de molienda, con un tiempo total de funcionamiento de 15 h. ${ }^{\text {ii }}$ Debido a que luego de la molienda gran

\footnotetext{
i El acero especial K (AISI D6) composición química característica de 85\%Fe, 12.4\%Cr, 2\%C, 0.35\%Mn y 0.25\%Si.

ii El tiempo de 15 horas escogido fue producto de un estudio de la cinética de molienda, el cual se presenta en el Capítulo 5.
} 
parte del material queda adherido a las paredes del cilindro se procedió a efectuar lavados con alcohol isopropílico. Estos lavados consistieron en agregar un pequeño volumen de alcohol en los cilindro y colocarlos nuevamente en el molino durante 3 minutos. Con este procedimiento se logró que el material que estaba adherido a las paredes quede suspendido en el alcohol, el cual fue depositado en un vidrio de reloj para que el alcohol se evapore y así, posteriormente, recuperar el material molido. Se efectuaron 2 lavados con el procedimiento antes indicado, consiguiendo rescatar la mayor parte del material (220 mg aprox.). En las preparaciones no se empleó ningún agente antiadherente como puede ser benceno $\left(\mathrm{C}_{6} \mathrm{H}_{6}\right)$ o metanol $\left(\mathrm{CH}_{3} \mathrm{OH}\right)$ para evitar la formación de carburos de hierro debido al carbono presente en los alcoholes. ${ }^{10}$

La incerteza, en la composición de todas las muestras preparadas está directamente asociada a la precisión de la balanza con la que se pesaron los metales. Una posible fuente de error en las concentraciones puede también provenir de contaminación con los materiales de los cilindros y esferas. Para asegurar la reproducibilidad de las muestras se realizaron más de una preparación para cada concentración presentada en esta Tesis. Para determinar el grado de contaminación al que pueden estar expuestas las aleaciones, se molió durante $15 \mathrm{~h}$ una muestra de Mn puro y posteriormente se le realizó una medida Mössbauer. Los resultados no mostraron indicios de Fe indicando que de existir contaminación ésta es inferior a la resolución experimental de la técnica.

\subsubsection{Fraccionamiento y montaje de muestras}

Terminada la molienda, se fraccionaron las muestras para ser analizada por Difracción de Rayos X (DRX), Espectroscopia de Absorción de Rayos X (XAS), Espectroscopía Mössbauer (EM) y medidas magnéticas (MM).

Para DRX se separaron $50 \mathrm{mg}$ de muestra los cuales se colocan en portamuestras de vidrio, especialmente diseñados para el difractómetro. En este caso la totalidad de la muestra pudo ser recuperada luego de realizar la medida. A continuación se muestra, en la figura 4.3, un esquema del montaje de la muestra para ser medida por DRX. 


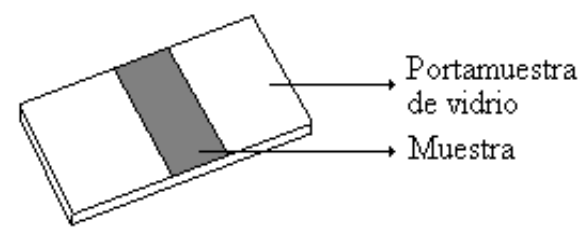

Figura 4.3: Esquema del soporte empleado para realizar las medidas de ditracción de rayos X.

Para realizar las medidas Mössbauer se depositaron $40 \mathrm{mg}$ de material en un portamuestras de acrílico de $2 \mathrm{~cm}$ de diámetro, colocando entre la muestra y el portamuestra una tira de papel de aluminio para asegura el contacto térmico con el soporte del criógeno para realizar las medidas a bajas temperaturas. A continuación se muestra, en la figura 4.4, un esquema del montaje empleado para realizar las medidas de EM.

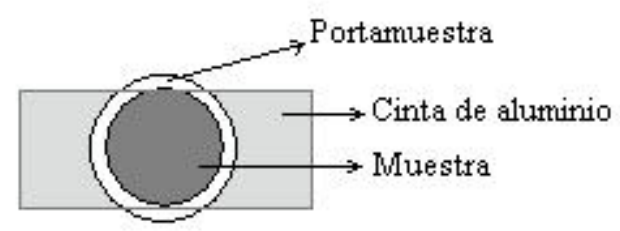

Figura 4.4: Esquema del montaje de una muestra para ser medida por espectroscopía Mössbauer.

Para las medidas magnéticas se colocaron entre 4 y $12 \mathrm{mg}$ (dependiendo de la respuesta magnética esperada) de muestra en una cápsula de gelatina, la cual a su vez, fue colocada en el interior de una pajilla plástica a $10 \mathrm{~cm}$ de su extremo superior. Es muy importante usar tanto cápsulas como pajillas incoloras ya que los pigmentos que se utilizan para colorear éstos poseen compuestos de hierro, que pueden afectar la medida magnética del material. Esta pajilla es acoplada a una varilla de acero inoxidable, la cual se introduce en el equipo y lleva a la muestra hasta la ubicación correcta para la medida. A continuación se muestra en la figura 4.5 un esquema del montaje de la muestra para realizar las medidas de magnetización. Es importante notar la presencia de un cerramiento de kapton ${ }^{\mathrm{iii}}$ en la parte inferior de la pajilla, como medida preventiva para evitar que, si por algún motivo se rompe la cápsula contenedora, el material no caiga dentro del equipo de medida.

\footnotetext{
iii Se utilizó una cinta de kapton para hacer el cerramiento debido a la muy baja respuesta magnética que este material presenta y su resistencia a las temperaturas de medida.
} 


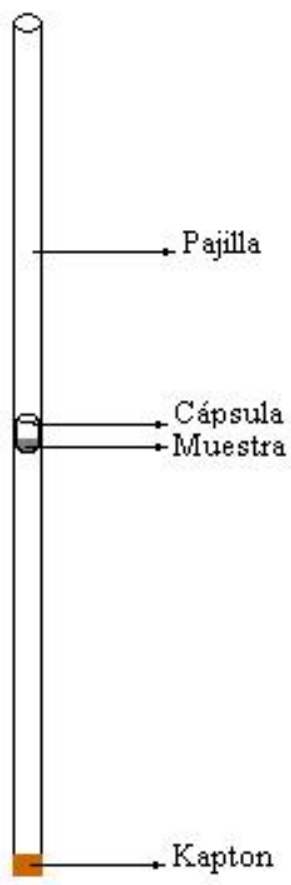

Figura 4.5: Esquema del montaje de la muestra para la realización de las medidas magnéticas.

Finalmente, la preparación de las muestras para realizar las medidas XAS consistió en pastillar la muestra. Para esto se mezcló en un mortero de ágata 40 mg de muestra con 200 mg de nitruro de boro y se los colocó en una prensa pastilladora, obteniendo un botón de material de $1 \mathrm{~cm}$ de diámetro, el cual se adosó con una cinta de kapton a un marco cuadrado de plástico de $5 \mathrm{~cm}$. A continuación, se muestra en la figura 4.6 un esquema del montaje de la muestra para ser medida por XAS.

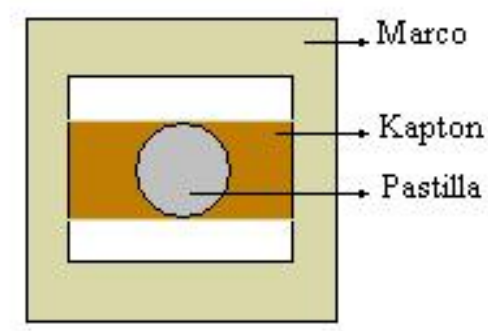

Figura 4.6: Esquema del montaje de la muestra para realizar las medidas de absorción de rayos X. 


\subsection{Difracción de Rayos X}

\subsubsection{Medida experimental}

Los difractogramas fueron realizados en el Laboratorio Nacional de Difracción (LANADI) de la Facultad de Ciencias Exactas, Universidad Nacional de La Plata.

Para la obtención de los difractogramas se utilizó un difractómetro Philips X'Pert Pro con geometría Bragg-Brentano en modo de adquisición de datos por paso, con un ánodo de $\mathrm{Cu}$ de longitud de onda promedio $\lambda=1,5418 \AA$. Se trabajó con una tensión de $40 \mathrm{kV}$ y una corriente de $40 \mathrm{~mA}$. La figura 4.7 muestra una fotografía del difractómetro empleado.

El difractómetro está provisto de un monocromador de haz difractado. Las ranuras de divergencia y recepción fueron de $1^{\circ}$ y $0,1^{\circ}$ respectivamente. Las medidas se realizaron en el rango de $2 \theta$ entre $20^{\circ}$ y $100^{\circ}$, con un paso de $0.02^{\circ}$ con 1 s por paso, excepto en la muestra con $\mathrm{x}=0.30$ donde este tiempo fue de $15 \mathrm{~s}$. Los datos obtenidos se guardaron en archivos para su posterior análisis.
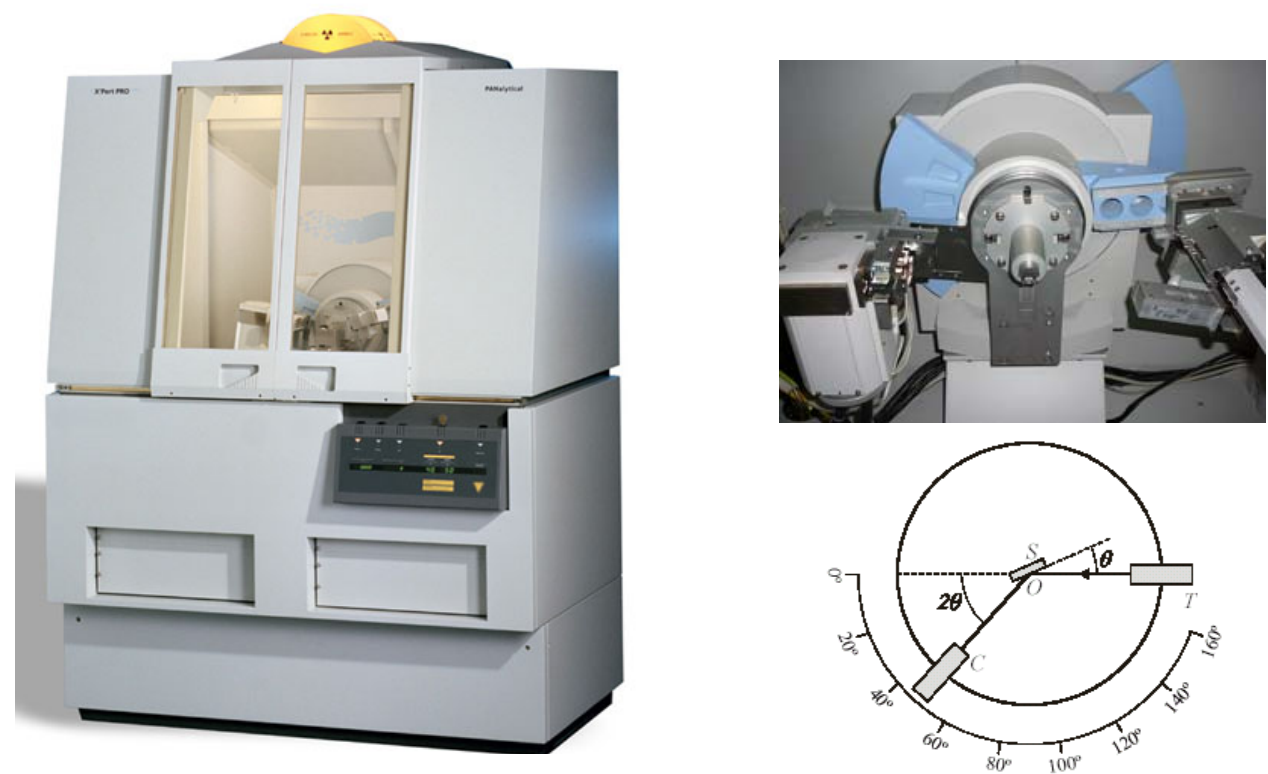

Figura 4.7: Difractómetro Philips $X$ ’Pert Pro utilizado para realizar las medidas de difracción de rayos X (izq). Fotografía del goniómetro del difractómetro junto con un esquema de la geometría de trabajo del instrumento (der). 


\subsubsection{Análisis de datos}

El análisis de los difractogramas fue llevado a cabo empleando los programas WinFit ${ }^{11} \mathrm{y}$ PowderCell. ${ }^{12}$ El primero de éstos permite reproducir el espectro de difracción sin entrada de ningún parámetro específico de la muestra. Para ello se propone que cada línea sea reproducida con un perfil de línea ya sea Gaussiana, Lorentziana, Voigtiana o Person VII. Cada uno de estos perfiles de ajuste está relacionado con la información que se quiere obtener de las líneas de difracción. En este caso se eligió un ajuste con un perfil Voigtiano el cual brindó la posición central del pico $\left(X_{c}\right)$ y su ancho integrado $(\beta)$. Ya que una línea Voigtiana es la convolución de un perfil Gaussiano y un perfil Lorentziano, del ajuste se obtiene, por lo general, un ancho medio integrado de cada perfil ( $\beta_{\mathrm{G}} \mathrm{y} \beta_{\mathrm{L}}$ ). Como se mostrará más adelante, los valores de $X_{c}$ y $\beta$ se pueden relacionar con los efectos que producen sobre el ancho de las líneas de difracción el tamaño de las cristalitas que difractan y las tensiones a las que se encuentran sometidos dichos cristales. Por otra parte, el programa PowderCell permite hacer un refinamiento del difractograma a partir de la simulación de las fases presentes. Con este refinamiento se obtiene información del porcentaje de las distintas fases, siendo también posible refinar los parámetros de red de cada fase.

\section{Tamaño de cristalita}

Para un material policristalino, con cristales libres de tensiones y suficientemente grandes, la teoría de difracción predice que las líneas deben ser extremadamente delgadas. En general, cuando se tiene una muestra policristalina la forma y el ancho del perfil de la línea están determinados por el tamaño, o distribución de tamaños, y por las imperfecciones que posea la cristalita.

Existen diversos métodos para determinar el tamaño de grano. ${ }^{13,14}$ Se describirá a continuación el método basado en la fórmula de Scherrer usado para analizar los cálculos de los resultados presentados en esta Tesis.

En 1918 Scherrer desarrolló una fórmula empírica que permite determinar el tamaño de cristalita a partir de la determinación de la posición y ancho integrado de un pico cualquiera del difractograma. ${ }^{13}$ Dicha fórmula tiene la siguiente expresión: 


$$
D=\frac{K \lambda}{\beta \cos (\theta)}
$$

donde $\lambda$ es la longitud de onda de la radiación con la que fue llevada a cabo a medida, $\theta$ la posición central del pico de difracción estudiado, $\beta$ el ancho integrado (en $2 \theta$ radianes) y $K$ es la denominada constante de Scherrer la cual depende de la forma del cristal y puede tomar valores entre $0.87 \mathrm{y} 1 .^{\text {iv }}$ El valor del ancho integrado $\beta$ debe ser corregido debido al propio ancho de línea que posee el difractómetro. Además, la forma de la corrección dependerá del tipo de perfil usado para el ajuste, siendo las relaciones usadas las siguientes:

Lorentziano:

$$
\beta_{\text {med }}=\beta_{D}+\beta_{\varepsilon}+\beta_{\text {ins }}
$$

Gaussiano:

$$
\beta_{\text {med }}^{2}=\beta_{D}^{2}+\beta_{\varepsilon}^{2}+\beta_{\text {ins }}^{2}
$$

donde $\beta_{\text {med }}$ es el ancho integrado obtenido del ajuste, $\beta_{D}$ la contribución del tamaño de cristalita, $\beta_{\varepsilon}$ la contribución de las tensiones y $\beta_{\text {ins }}$ la contribución intrínseca del difractómetro.

En caso de elegir un perfil Voigtiano es necesario primero deconvolucionarlo en sus componentes Gaussiana y Lorentziana, antes de hacer la corrección instrumental.

${ }^{\text {iv }}$ En este trabajo la constante de Scherrer fue tomada igual a la unidad. 


\subsection{Espectroscopia de Absorción de Rayos X}

\subsubsection{Medida experimental}

Las medidas XAS fueron realizadas en el Laboratorio Nacional de Luz Sincrotrón (LNLS) en Campinas, Brasil (figura 4.8). Se trabajó en la línea XAFS1 en donde se obtuvo el espectro de absorción en el borde $K$ del $\mathrm{Cu}$ a temperatura ambiente. Los espectros fueron adquiridos en la configuración de fluorescencia (figura 4.9) usando un arreglo de 15 detectores de Ge. La corriente nominal con la que se trabajó fue de $200 \mathrm{~mA}$ y se realizaron de 3 a 4 barridos por muestra, los cuales fueron promediados, en un rango de energía que fue de 8880 a $10030 \mathrm{eV}$.

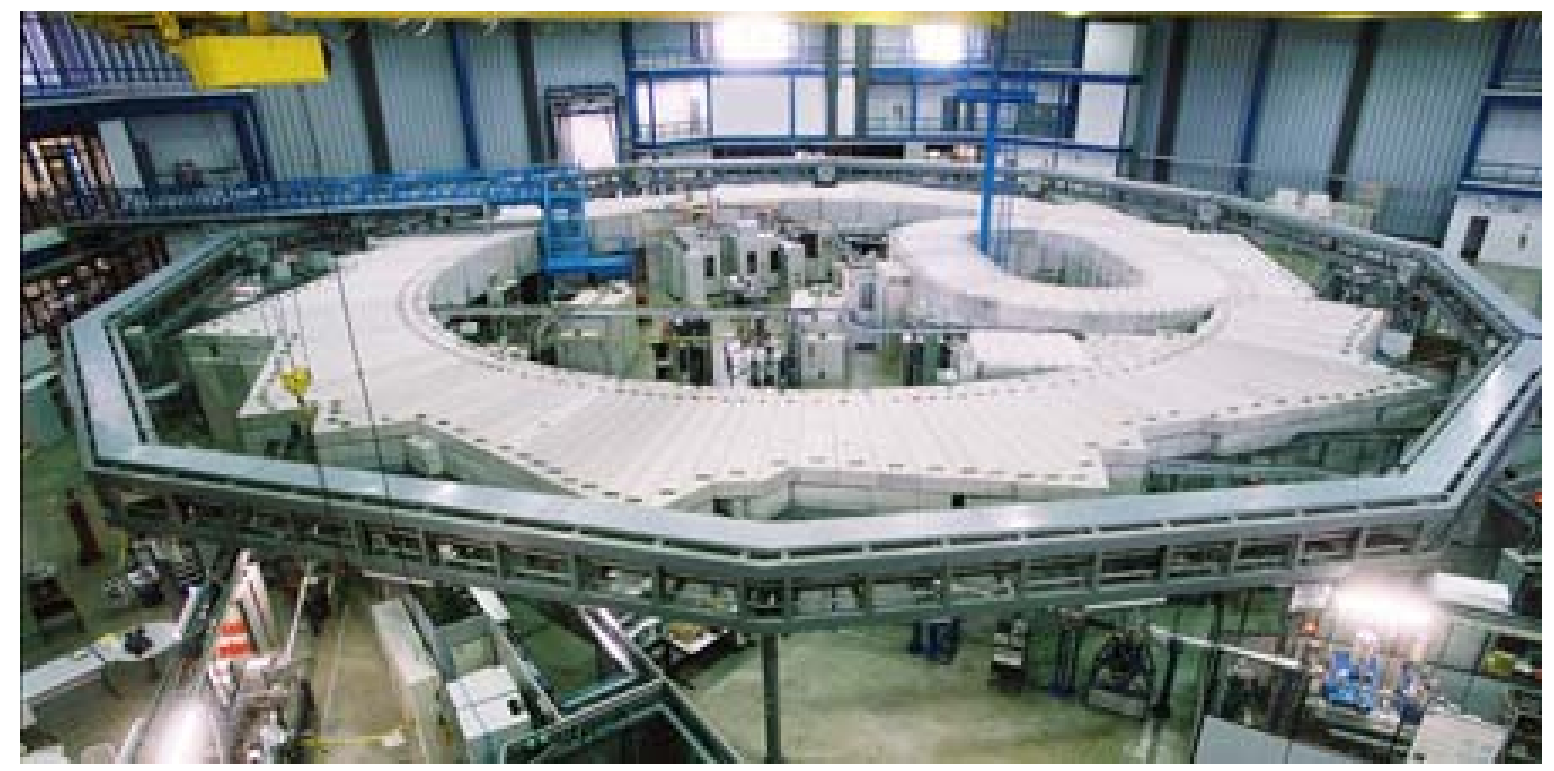

Figura 4.8: Fotografía del anillo acelerador de electrones ubicado en el Laboratorio Nacional de Luz Sincrotrón. 


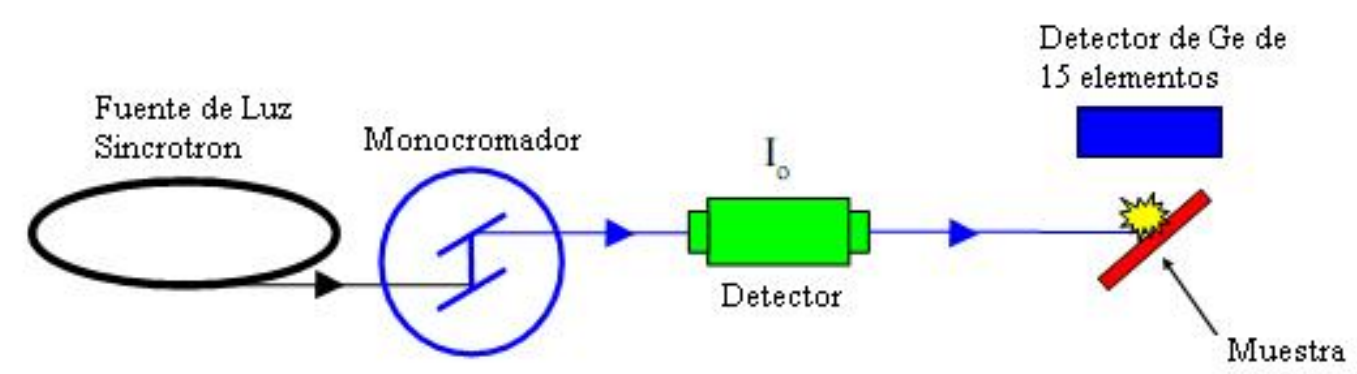

Figura 4.9: Esquema simplificado del arreglo empleado para realizar las medidas de absorción de rayos $\mathrm{X}$ para el caso de la detección por fluorescencia.

Se realizaron medidas por transmisión en el borde K del Fe con la muestra pastillada, pero la rugosidad de la superficie hizo que el espectro tuviera mucho ruido en la región EXAFS por lo que en la sección de resultados se presentará la información obtenida para la región XANES. Por otra parte tampoco fue posible realizar las medidas por fluorescencia en este borde debido a que por ser el Fe el material mayoritario, los efectos de autofluorescencia afectarán al espectro obtenido.

\subsubsection{Análisis de datos}

La señal EXAFS es producto de la suma de ondas dispersadas debidas a los diferentes tipos de entornos del átomo absorbente. ${ }^{15}$ Esta suma de ondas es gobernada por la cantidad y tipo de átomos vecinos al átomo absorbente, y por las distancias entre los mismos. En el espacio recíproco $k$ la onda está descripta por:

$$
\chi(k)=\sum_{j} \frac{S_{0}^{2} N_{j} F_{j}(k) \exp \left(-2 \sigma_{j}^{2} k^{2}\right) \exp \left(-2 r_{j} / \lambda_{j}(k)\right)}{k r_{j}^{2}} \operatorname{sen}\left(2 k r_{j}+\phi(k)\right)
$$

donde $S_{0}{ }^{2}$ es denominado factor de atenuación, $N_{j}$ es el número de vecinos del átomo $j, F_{j}$ es la amplitud de retrodispersión, $\sigma^{2}$ es el factor de Debye-Waller (asociado a vibraciones térmicas y desorden), $r_{j}$ es la distancia al átomo dispersor y $\phi$ es el corrimiento de fase experimentado por el fotoelectrón. El factor $\exp \left(-2 r_{j} / \lambda_{j}(k)\right)$ tiene en cuenta las pérdidas inelásticas en el proceso 
dispersivo (debido a los átomos vecinos al átomo absorbente y al medio entre los mismos) siendo $\lambda$ el camino libre medio del fotoelectrón entre el átomo absorbente y el dispersor.

Para el análisis de los espectros fue, primeramente, necesario realizar la sustracción de la línea de base a los espectros y normalizarlos considerando la región extendida de los mismos. Las oscilaciones de la estructura fina $\chi(\mathrm{k})$ de cada espectro fueron analizadas haciendo la transformada de Fourier de los mismos y seleccionando la porción del espectro correspondiente a la primera esfera de coordinación. A continuación, se describe el procedimiento para aislar la oscilación EXAFS de un espectro de absorción de rayos X.

\section{Aislamiento de la oscilación EXAFS}

El tratamiento de los datos EXAFS consiste en diferentes etapas las cuales fueron realizadas empleando el programa Athena, mientras que para el ajuste de los espectros se usó el programa Arthemis, ambos pertenecientes el paquete de software IFEFFIT. ${ }^{16}$ La primera es aislar la oscilación $\chi(k)$. Para ello se resta el fondo que se encuentra en la región anterior al borde de absorción, usualmente ajustado con una curva del tipo victoreana modificada. Luego se procede a normalizar el espectro a la región donde las oscilaciones ya están atenuadas. El paso siguiente es determinar la energía del borde para luego poder hacer la transformación al espacio recíproco, dicha transformación se realiza empleando la siguiente relación:

$$
k=\sqrt{\left(\frac{8 \pi^{2} m}{h^{2}}\right)\left(h v-E_{\text {borde }}\right)}
$$

donde $m$ es la masa del electrón y $h$ la constante de Plank. Por lo general, para la elección de la energía de borde $\mathrm{E}_{\text {borde, }}$ se utiliza el punto de inflexión en la región de absorción del espectro, determinado por el máximo en la primera derivada del mismo con respecto a la energía. Luego de convertir los datos al espacio $k$, se procede a restar la contribución del borde de absorción para lo cual se ajusta un polinomio, por lo general, con uno de grado dos es suficiente, para obtener la contribución de la región EXAFS. A continuación, en la figura 4.10, se muestran gráficos que representan las diferentes etapas del tratamiento mencionadas. 

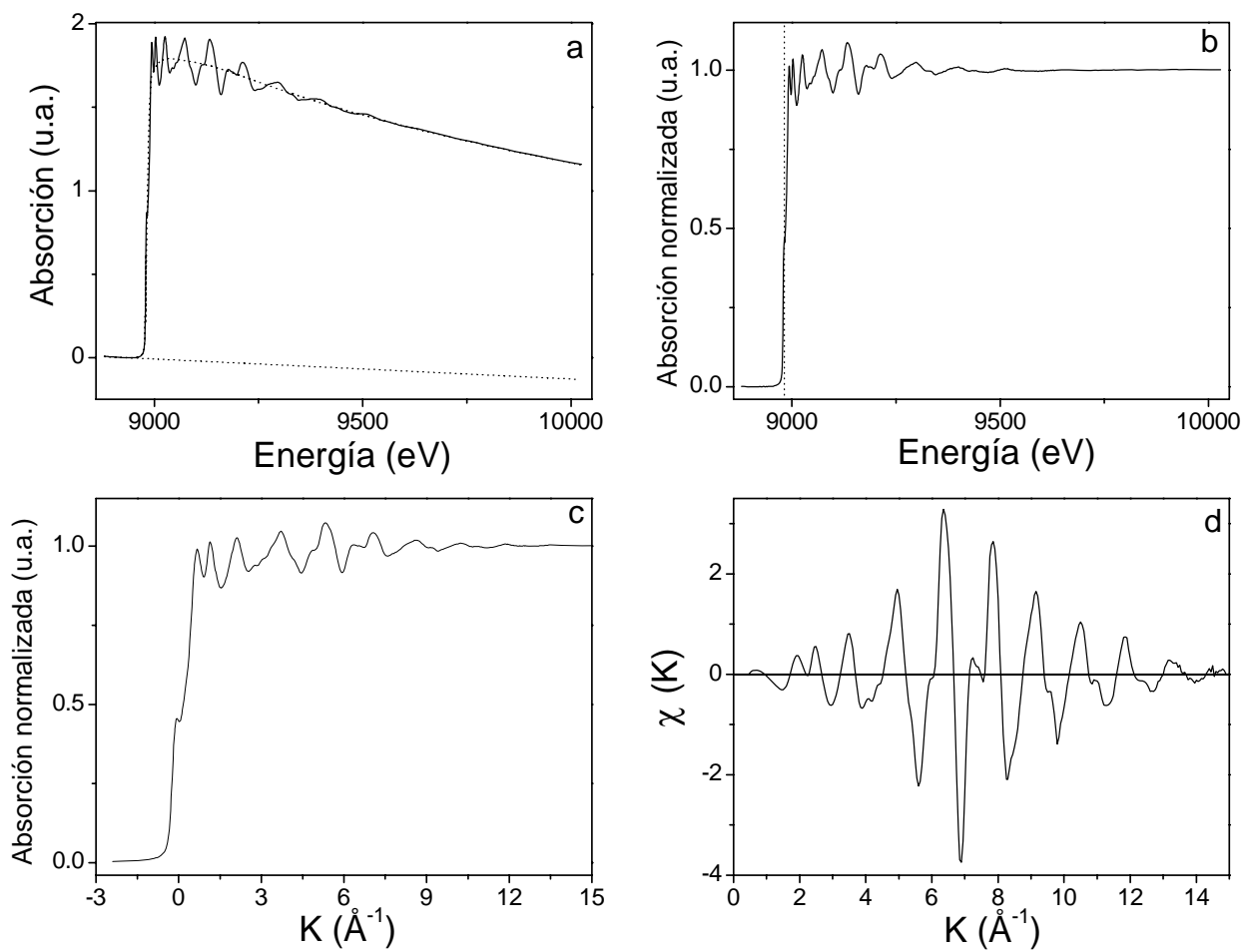

Figura 4.10: Etapas del tratamiento de la señal EXAFS. a) restado de línea de base, b) normalizado, c) determinación de energía de borde y d) remoción de la absorción atómica.

La segunda etapa en el tratamiento de los datos, consiste en realizar la trasformada de Fourier (TF) del espectro. La TF de $\chi(k)$ resulta en una función de distribución radial, similar a la que se muestra a continuación en la figura 4.11:

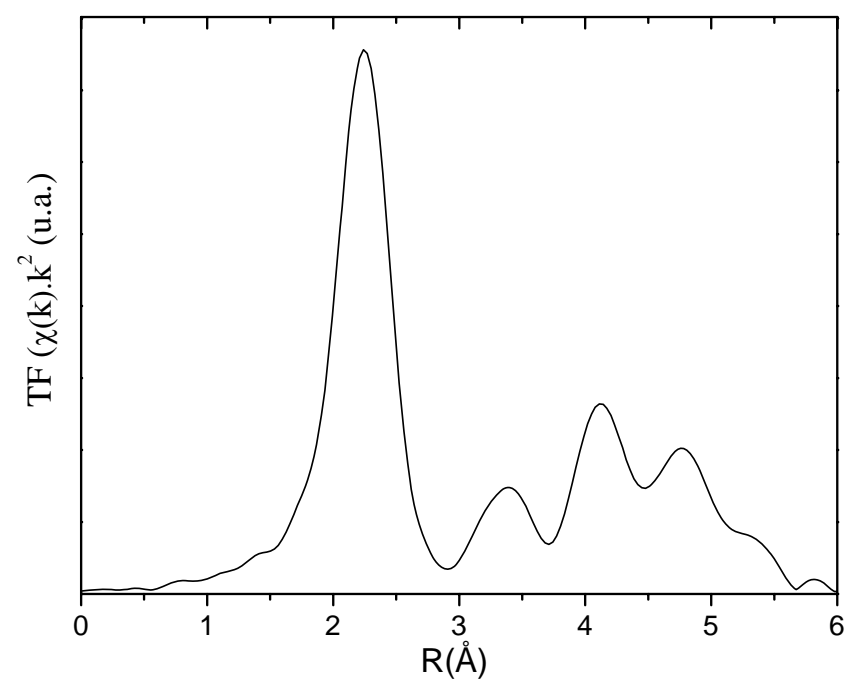

Figura 4.11: Transformada de Fourier del espectro de absorción de rayos X para el Cu medido en el borde K. 
Esta función de distribución radial está definida en el espacio de las coordenadas, las cuales están medidas con respecto a la posición del átomo absorbente. La distancia observada en la TF es alrededor de 0.2 a $0.5 \AA$ menor que la distancia real debido a la fase presente en la función oscilatoria en $\chi(k)$. La TF puede realizarse con diferentes pesos en $k$ (multiplicando por $k^{n}$ con $n$ un número entero mayor que 1), ésto permite discriminar entre diferentes átomos dispersores en una misma región, ya que, elementos menos másicos dispersarán a menores valores de $k$, mientras que elementos más pesados, como metales, producirán una dispersión mucho más significativa a valores de $k$ altos. En el presente caso ya que los átomos dispersores son todos metales, se optó por usar una TF pesada con $k^{2}$.

Posteriormente, se procede a aislar las contribuciones de las distintas esferas de coordinación, proceso que se conoce como filtrado. Para ello se realiza la TF inversa seleccionando la región de interés del espectro. Este filtrado es útil para comenzar con el ajuste del espectro ya que, intentar reproducir inicialmente las contribuciones de 3 o 4 esferas actuando simultáneamente, resultaría muy complicado. Este procedimiento también se debe realizar en una muestra patrón, pues nos permitirá obtener estándares para la amplitud y la fase, necesarios para la posterior reproducción de los espectros a analizar.

Para realizar el ajuste de los espectros experimentales primeramente se debe obtener información del elemento correspondiente al borde de absorción que se esté estudiando. Esta información se puede conseguir del ajuste de un espectro de una muestra patrón del elemento en cuestión, o por cálculos de primeros principios. En este caso el valor del factor de atenuación $S_{0}{ }^{2}$ fue calculado por ambos métodos, obteniéndose en ambos casos resultados idénticos dentro del error del ajuste. Los valores de la amplitud y la fase fueron también calculados empleando métodos de primeros principios. Una vez que se cuenta con esta información se procede a trabajar con los espectros de las muestras a estudiar.

En el modelo propuesto para realizar los ajustes se trató a los átomos de Mn y Fe como indistinguibles, ya que dadas sus similitudes químicas la dispersión que producirán uno u otro al fotoelectrón será similar. Considerando esta aproximación, se propuso un modelo por capas. La primer capa está conformada por átomos de $\mathrm{Cu}$ a una distancia de $2.54 \AA$, mientras que la segunda capa estaba conformada por átomos de Fe a la misma distancia, de esta forma ambas capas están representando la primera esfera de coordinación del átomo absorbente. Dado que por difracción de rayos $\mathrm{X}$ se sabe que la fase mayoritaria en todas las muestras posee una estructura $f c c$, se colocó una restricción al número de primeros vecinos acorde a dicha fase, forzando a que éste sea igual a 12. Los valores de los factores de Debye-Waller (FDW) usados en la capa de $\mathrm{Cu}$ y en la de Fe son los predichos por el modelo de Einstein. ${ }^{17}$ 


\subsection{Espectroscopía Mössbauer}

\section{Introducción}

El efecto Mössbauer es excelente en cuanto a la posibilidad de determinar el estado químico de la sonda, las fracciones de los distintos sitios en los cuales está ubicada, etc. Cada sólido, que posea un isótopo Mössbauer, tendrá asociado un espectro característico del cual se podrán deducir propiedades electrónicas, magnéticas y químicas del mismo. La Espectroscopía Mössbauer permite un análisis no destructivo de muestras que contengan más de una fase, siendo el método también adecuado para el estudio de sólidos amorfos o materiales micro o nanocristalinos.

La espectroscopía Mössbauer es una técnica basada en la emisión y absorción resonante de rayos $\gamma$ emitidos por una fuente radioactiva. Cuando ocurre la emisión de un rayo $\gamma$ por parte de la fuente con una probabilidad $p_{1}$ aquellos núcleos de la muestra que la absorban resonantemente con una probabilidad $p_{2}$ inmediatamente después, reemitirán rayos $\gamma$ restándoselos con una probabilidad $p_{1} \mathrm{x} p_{2}$ al haz que está incidiendo en el detector, y en consecuencia se tendrá una disminución en el número de cuentas en el detectadas. Si el entorno químico del núcleo que emite el fotón $\gamma$ no es exactamente igual al del núcleo que puede absorberlo las perturbaciones hiperfinas afectaran a los niveles de energía nucleares del absorbente de manera distinta que a los de la fuente. En estos casos para lograr que se produzca la absorción resonante se deberá modificar la energía del rayo $\gamma$, lo que se logra moviendo la fuente o el absorbente, como se indica en la figura 4.12:

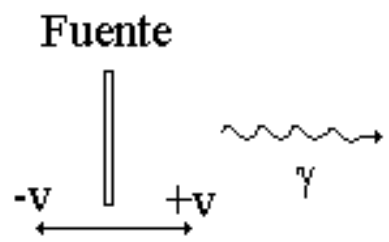

Absorbente

Detector
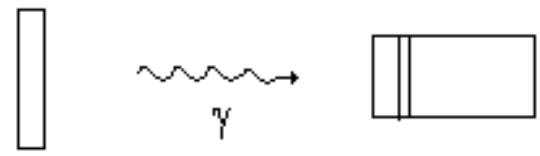

Figura 4.12: Esquema simplificado del arreglo experimental empleado para realizar las medidas de espestroscopía Mössbauer en geometría de transmisión. 
Por medio del dispositivo de la figura 4.12 es posible cambiar la energía de la radiación incidente en cantidades tan pequeñas como se desee haciendo uso del efecto Doppler. Por lo tanto, si la fuente está moviéndose respecto al sistema de laboratorio con una velocidad v, se obtendrá, a primer orden, una variación de la energía del rayo $\gamma$ dada por:

$$
\delta E=\frac{\mathrm{v}}{c} E_{\gamma},
$$

donde, c es la velocidad de la luz en el vacío y $\mathrm{E}_{\gamma}$ la energía del fotón $\gamma$ con la fuente en reposo. Unos pocos mm/s son suficientes para observar una disminución del conteo en el detector, lo que está implicando la aparición de la resonancia.

Los parámetros hiperfinos que se determinan empleando la espectroscopía Mössbauer son los siguientes:

\section{- Corrimiento isomérico}

El corrimiento isomérico mide la diferencia de densidades electrónicas $s$ dentro del volumen nuclear entre la fuente y el absorbente. Esta interacción no existiría si el núcleo fuera puntual, y depende también de la variación del radio nuclear entre los estados excitados y el fundamental. El corrimiento isomérico no levanta la degeneración de los niveles nucleares sino que produce un corrimiento de una línea no desdoblada con respecto del cero de velocidad. Hay también otros factores de menor importancia que contribuyen al corrimiento isomérico como el corrimiento Doppler de segundo orden.

\section{- Desdoblamiento cuadrupolar}

El desdoblamiento cuadrupolar es debido a por la interacción entre el momento cuadrupolar eléctrico del núcleo y el gradiente de campo eléctrico creado por distribuciones de carga no simétricas en la posición del núcleo. 


\section{- Interacción hiperfina magnética}

Ésta es debida a la interacción entre el momento magnético nuclear $\mu$ y un campo magnético que no fluctúa durante el tiempo de vida media del estado excitado. Si el campo magnético variara muy rápidamente el efecto promediará a cero y el espectro no diferirá del no perturbado. Para tiempos intermedios se comienza a observar una relajación del espectro, hasta que para tiempos muy largos se logra la situación estática en la que se observará la interacción. El desdoblamiento magnético hiperfino permite determinar el campo magnético efectivo que actúa sobre el núcleo. Esta propiedad permite distinguir sustancias que se encuentran en un régimen paramagnético de aquellas que se encuentran en un estado magnético ordenado, pudiéndose medir las temperaturas de ordenamiento del sistema y la variación del campo hiperfino con la temperatura. Puesto que el efecto Mössbauer es producto de sumar contribuciones individuales de cada átomo, es posible obtener la magnetización de cada subred que compone la muestra lo cual no se podría hacer con técnicas macroscópicas, cuyos resultados son el promedio de una propiedad extendida a toda la muestra.

\subsubsection{Medida experimental}

Los espectros Mössbauer se adquirieron utilizando la geometría de transmisión para todas las muestras estudiadas en este trabajo. El equipo consta de dos partes (figura 4.13): la primera contiene un mecanismo electromecánico que hace posible el movimiento de la fuente, lo cual proporciona a la radiación $\gamma$ la energía suplementaria necesaria para la resonancia de cada una de las transiciones posibles entre el nivel fundamental y los excitados del núcleo del átomo sonda. Por otro lado, se tiene el sistema de detección de los rayos $\gamma$. Debido a que ningún detector tiene la resolución necesaria para resolver una transición de otra, que difieren entre sí del orden de los $10^{-8}$ eV, la discriminación se hace por la simultaneidad del rayo $\gamma$ y la velocidad a la que se está moviendo la fuente en cada instante. Es por esto que la exactitud de sincronización, entre el mecanismo electromecánico y la adquisición de los datos, es uno de los parámetros fundamentales en estos equipos. La rama de detección consta del detector propiamente dicho (contador proporcional), de un preamplificador, un amplificador y de un selector de pulsos. El selector de pulsos es el que genera una señal lógica cuando son detectados rayos $\gamma$ correspondientes a la transición de interés, filtrando todos los otros posibles rayos presentes en la fuente provenientes de las transiciones entre otros niveles. 


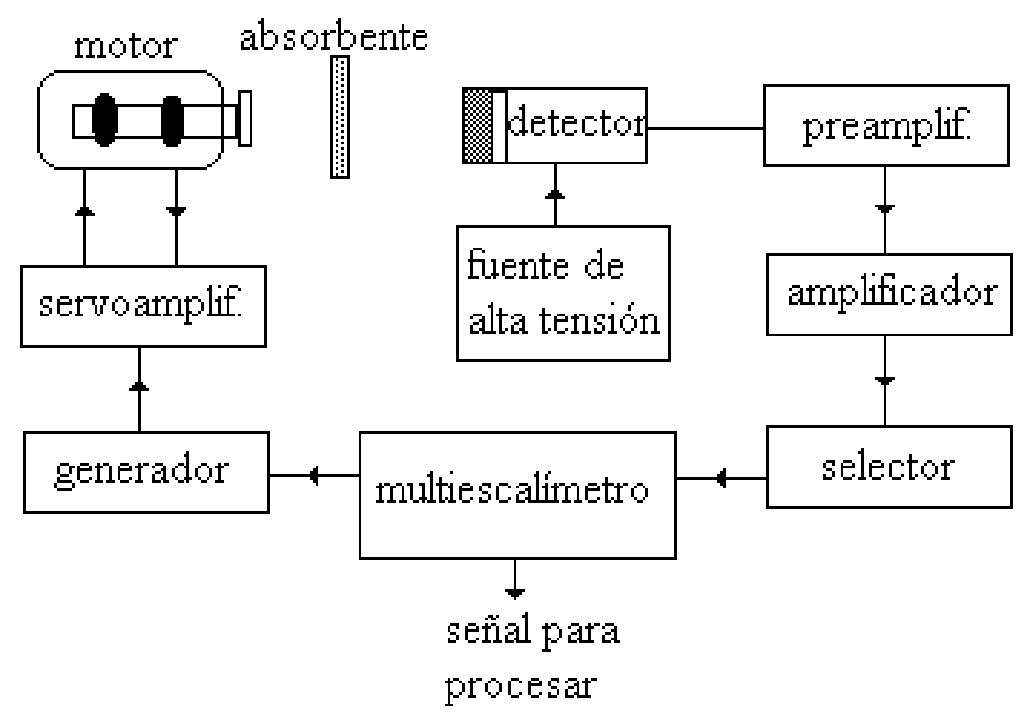

Figura 4.13: Diagrama de la disposición experimental de la electrónica de comando de un espectrómetro Mössbauer.

Todos los espectros Mössbauer presentados en este trabajo fueron tomados en el Laboratorio de Aplicaciones del Efecto Mössbauer y Magnetismo (LAEMM) del Departamento de Física de la Facultad de Ciencias Exactas, Universidad Nacional de La Plata. Se utilizó una fuente de ${ }^{57} \mathrm{Co} / \mathrm{Rh}$ de una intensidad de $5 \mathrm{mCi}$ aproximadamente, y los espectros fueron tomados en un espectrómetro convencional de aceleración constante de 512 canales.

Para la adquisición de los espectros se cubrió un intervalo de alta velocidad entre $-8 \mathrm{~mm} / \mathrm{s}$ y $+8 \mathrm{~mm} / \mathrm{s}$ y uno de baja velocidad $-4 \mathrm{~mm} / \mathrm{s}$ y $+4 \mathrm{~mm} / \mathrm{s}$. La calibración en velocidades fue realizada con una lámina de $\alpha$-Fe de $12 \mu \mathrm{m}$ de espesor. Los espectros fueron analizados con distribuciones de campos magnéticos hiperfinos, para lo cual se realizó con el programa Vandenberghe. ${ }^{18}$

Para realizar las medidas Mössbauer a bajas temperaturas se utilizó un criógeno de ciclo cerrado de He que permitió alcanzar temperaturas de $21 \mathrm{~K}$. 


\subsubsection{Análisis de datos}

Los espectros Mössbauer para todas las concentraciones estudiadas constan principalmente de una señal central ancha, por lo que dichos espectros pueden, a priori, ser reproducidos empleando diversos criterios. Según la bibliografía ${ }^{19,20}$ la aleación de $\mathrm{Fe}_{79} \mathrm{Mn}_{21}$ presenta una temperatura de orden antiferromagnético que se encuentra a una temperatura de $354 \mathrm{~K}$ lo que determina que a temperatura ambiente se observe una señal magnética de bajo campo hiperfino (2 T aproximadamente) no resuelta. Empleando esta información, en una primera etapa se intentó reproducir los datos experimentales empleando un ajuste con tres sitios magnéticos, los cuales representarían tres entornos distintos del átomo absorbente. Estos ajustes mostraban que los anchos de línea necesarios para lograr una buena reproducción del espectro deberían ser del orden de 0.45 $\mathrm{mm} / \mathrm{s}$, lo cual es un valor muy grande comparado con el ancho de línea experimental esperado para el ${ }^{57} \mathrm{Fe}(0.25 \mathrm{~mm} / \mathrm{s})$. Este último argumento llevó a escoger que la forma más indicada para la reproducción de los espectros era emplear una distribución de sitios magnéticos. Para dicho fin se utilizó el programa Vandenberghe. ${ }^{18}$ Una de los principales beneficios que presenta este programa es que permite realizar un ajuste empleando distribuciones de campos de forma arbitraria. Otra propiedad interesante que posee es que permite fijar o no la probabilidad a campo cero, lo cual puede ser útil si se tiene una contribución paramagnética, además de la magnética, las cuales posean corrimientos isoméricos similares.

Particularmente todos los espectros fueron reproducidos con distribuciones de campos magnéticos hiperfinos a los cuales se les permitió tener una probabilidad distinta de cero a campo nulo, además de la posibilidad de correlacionar el valor del campo hiperfino con el corrimiento isomérico. Todos los espectros Mössbauer están graficados sin hacer la corrección por la matriz de $R h$ en la que se encuentra la fuente, mientras que los valores de los corrimientos isoméricos obtenidos de los ajustes están referidos al $\alpha$-Fe. 


\subsection{Medidas Magnéticas}

\subsubsection{Medida experimental}

Las medidas magnéticas que se presentan son de tres tipos a saber: medidas de susceptibilidad alterna, medidas de magnetización en función de la temperatura y medidas de magnetización en función del campo magnético.

Las medidas de susceptibilidad AC fueron realizadas en el susceptómetro LakeShore 7130 perteneciente al Departamento de Física de la Facultad de Ciencias Exactas de la Universidad Nacional de La Plata. Las condiciones experimentales fueron las siguientes; se observó la variación de la susceptibilidad en función de la temperatura en el rango de $20 \mathrm{~K}$ a $320 \mathrm{~K}$, con una amplitud del campo magnético alterno 1 Oe a una frecuencia de $825 \mathrm{~Hz}$. Por otra parte se realizaron medidas haciendo barridos en frecuencia y en presencia de campos magnéticos estáticos. Todas las medidas fueron realizadas primeramente enfriando la muestra hasta la temperatura más baja y luego se comenzó a calentar simultáneamente que se realizaba la medida de la susceptibilidad. Para realizar las medidas a una determinada frecuencia se realizaron rampas de calentamiento con una velocidad continua de $4 \mathrm{~K} / \mathrm{min}$ y los datos fueron tomados cada $2 \mathrm{~K}$. Cuando se realizaron los barridos en frecuencia, debido a que es imprescindible que la temperatura correspondiente a las diversas frecuencias sea la misma, se procedió a estabilizar la temperatura en cada paso. Luego se procedió a medir la susceptibilidad a los valores de frecuencia deseados, para una vez terminado este procedo continuar con la siguiente temperatura y así siguiendo.

Las restantes medidas magnéticas se realizaron en el sistema de medición de propiedades magnéticas (MPMS) y el sistema de medición de propiedades físicas (PPMS) pertenecientes a las Red Nacional de Magnetismo (RN3M), los cuales se encuentran en el Laboratorio de Bajas Temperaturas en la Facultad de Ciencias Exactas y en el Laboratorio de Sólidos Amorfos de la Facultad de Ingeniería, respectivamente, de la Universidad Nacional de Buenos Aires. Las figura 4.14 muestra fotografías de los distintos equipos empleados para realizar las medidas magnéticas, mientras que en la figura 4.15 se puede observar un corte transversal del cilindro criogénico junto con un detalle de la región contenedora de la muestra y la varilla portamuestra. 

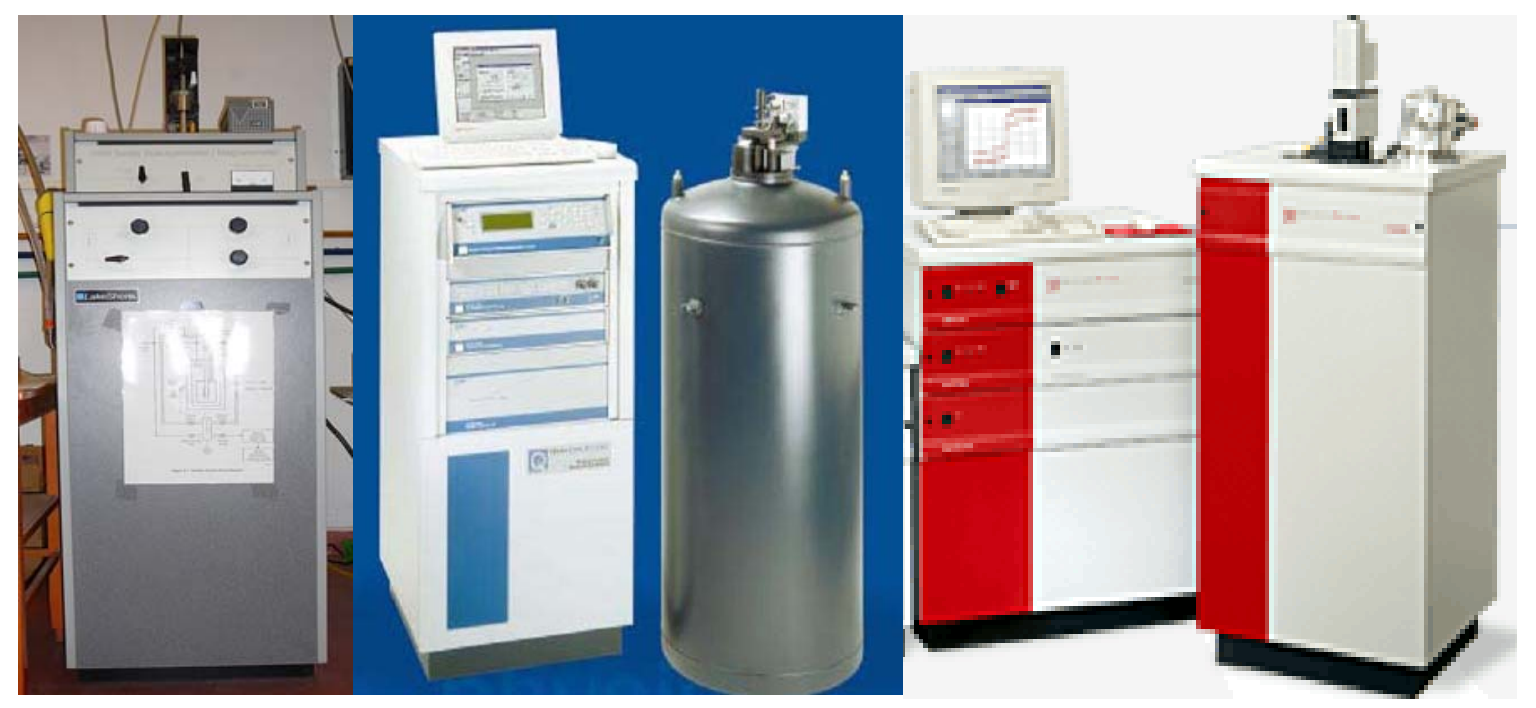

Figura 4.14: Fotografías de los equipos de magnetismo: Susceptómetro (izq.), PPMS (centro) y MPMS (der.).
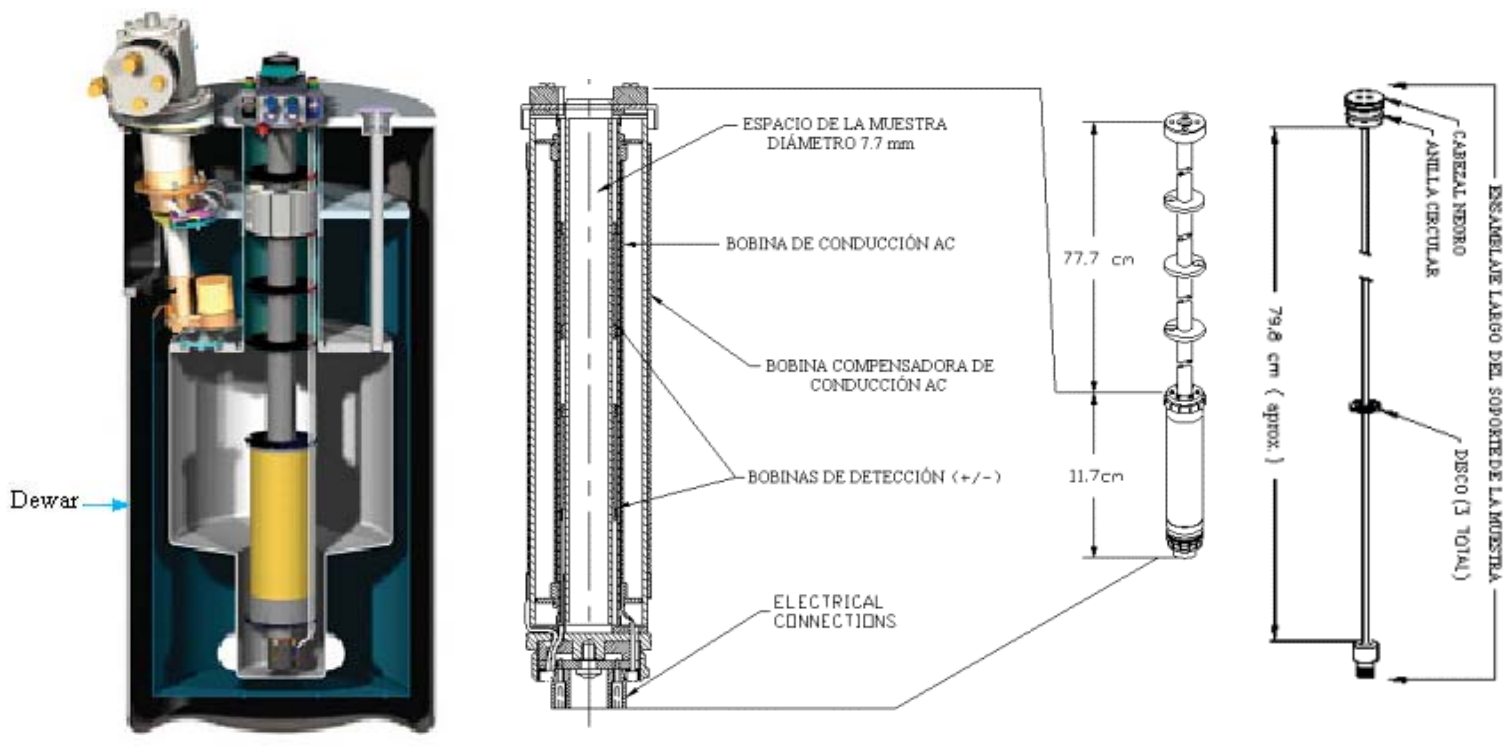

Figura 4.15: Corte transversal del cilindro criogénico del dispositivo PPMS (izq). Detalle de la región contenedora de la muestra donde se encuentran las bobinas de excitación y medición (centro). Varilla para soporte del portamuestra (der).

La magnetización en función de la temperatura fue medida en dos configuraciones distintas. En la primera se enfrió la muestra sin campo magnético aplicado hasta la temperatura a la cual se quiere comenzar a medir, luego se aplicó un campo magnético constante y se comenzó a subir la temperatura a la vez que se registró el valor de la magnetización de la muestra. Esta configuración de medida es llamada ZFC del inglés Zero Field Cooling o enfriado sin campo. La segunda 
configuración es similar a la anterior con la diferencia que en el proceso de enfriado está presente el campo magnético con el que luego se va a realizar la medida, este tipo de medida es llamada FC del inglés Field Cooling o enfriado con campo. En las muestras se realizó este tipo de medidas en un intervalo de temperaturas de $5 \mathrm{~K}$ a $300 \mathrm{~K}$ con campos de 20 Oe y 100 Oe. Para la muestra $\left(\mathrm{Fe}_{79} \mathrm{Mn}_{21}\right)_{70} \mathrm{Cu}_{30}$ además se realizaron medidas con campos de 200 Oe, 400 Oe y 4000 Oe. Las rampas de calentamiento usadas fueron de $4 \mathrm{~K} / \mathrm{min}$ registrando la magnetización cada $4 \mathrm{~K}$.

Por último la medida de magnetización en función del campo aplicado, la cual comúnmente se conoce como ciclo de histéresis fue medida a diversas temperaturas con campos magnéticos de hasta 50 kOe. En este caso también se hicieron medidas a baja temperatura habiendo enfriando al sistema en presencia de un campo magnético estático.

\subsubsection{Análisis de datos}

Para el análisis de los ciclos se cuenta con diversos modelos que pueden ser empleados dependiendo de las propiedades de las muestras estudiadas. Para una muestra ferromagnética que presenta un comportamiento histerético una de las expresiones más usadas es la siguiente: ${ }^{21}$

$$
M_{F}=M_{S}\left[\operatorname{Arctg}\left(\frac{H \pm H_{c}}{H_{c}} \operatorname{tg} \frac{S}{\pi}\right)\right]
$$

donde las magnitudes a determinar son la magnetización de saturación $\left(\mathrm{M}_{\mathrm{s}}\right)$, el campo coercitivo $\left(\mathrm{H}_{c}\right)$ y la relación entre la magnetización de remanencia y saturación (S). El doble signo presente en la expresión 4.7 es para contemplar ambas ramas del ciclo de histéresis.

Otra expresión útil para la reproducción de los ciclos es la dada por la ecuación de Langevin ${ }^{22}$ deducida en el Capítulo 3:

$$
M_{L}=M_{S L}\left[\operatorname{Cotgh}\left(\frac{\mu \cdot H}{k_{B} \cdot T}\right)-\frac{k_{B} \cdot T}{\mu \cdot H}\right]
$$

donde $\mathrm{M}_{\mathrm{SL}} \mathrm{y} \mu$ serán interpretados como la magnetización de saturación y el momento magnético asociados a ésta contribución, respectivamente. Este tipo de curvas son por lo general empleadas para representar a sistema de partículas con comportamiento superparamegnético como se 
describió en el Capítulo 3, pero también se ha empleado para sistemas de partículas antiferromagnéticas con momentos magnéticos no compensados en su superficie. ${ }^{23}$

Por último algunas veces es necesario contar con una función lineal la cual contemple un comportamiento diamagnético, paramagnético o antiferromagnético. La expresión usada para tal contribución viene dada por:

$$
M_{A}=\chi_{A} \cdot H
$$

donde el parámetro a determinar es la susceptibilidad magnética $\chi_{\mathrm{A}}$.

Para la reproducción de los ciclos fueron analizados varios modelos. Los modelos más simples consistentes en la combinación de dos de las contribuciones presentadas anteriormente no fueron capaces de reproducir adecuadamente los ciclos. Por este motivo se empleo un modelo que fuese la suma de las contribuciones 4.7 a 4.9, resultando la siguiente expresión:

$$
M=M_{F}+M_{L}+M_{A}=M_{S}\left[\operatorname{Arctg}\left(\frac{H \pm H_{c}}{H_{c}} \operatorname{tg} \frac{S}{\pi}\right)\right]+M_{S L}\left[\operatorname{Cotgh}\left(\frac{\mu \cdot H}{k_{B} \cdot T}\right)-\frac{k_{B} \cdot T}{\mu \cdot H}\right]+\chi_{A} \cdot H
$$

Se observó que la parte central de los ciclos medidos a bajas temperaturas presentaban una curvatura fuera de lo común, especialmente para las muestras con mayor concentración de Cu. Se realizaron las derivas de los ciclos de histéresis para ver si existía una segunda fase magnética la que no estaba siendo contemplada con los modelos anteriores. Luego de verificar la existencia de una segunda fase magnéticamente ordenada de baja temperatura, se propuso que para la reproducción de estos ciclos el siguiente modelo:

$$
M=M_{F 1}+M_{F 2}+M_{A}=M_{s 1}\left[\operatorname{Arctg}\left(\frac{H \pm H_{c 1}}{H_{c 1}} \operatorname{tg} \frac{S_{1}}{\pi}\right)\right]+M_{s 2}\left[\operatorname{Arctg}\left(\frac{H-H_{c 2}}{H_{c 2}} \operatorname{tg} \frac{S_{2}}{\pi}\right)\right]+\chi_{A} \cdot H
$$

donde la contribución de Langevin fue reemplazada por una segunda contribución ferromagnética con parámetros $\mathrm{M}_{\mathrm{S} 2}, \mathrm{H}_{\mathrm{C} 2}$ y $\mathrm{S}_{2}$, habiendo empleado el subíndice 2 para diferenciarlos de los parámetros correspondientes a la primer interacción ferromagnética. 


\subsection{Referencias}

\footnotetext{
${ }^{1}$ B.D. Cullity y S.R. Stock, en “Elements of X-Ray Diffraction”, 3 ra edición, Prentice Hall, (2001).

${ }^{2}$ V. Pecharsky y P. Zavalij, en "Fundamentals of Powder Diffraction and Structural Characterization of Materials”, $2^{\text {da }}$ edición, Springer, (2008).

3 G. Bunker, en "Introduction to XAFS: A Practical Guide to X-ray Absorption Fine Structure Spectroscopy”, $1^{\text {ra }}$ edición, Cambridge University Press, (2010).

${ }^{4}$ G. Maddock, en "Mössbauer Spectroscopy: Principles \& Applications of the Techniques", Horwood Publishing Limited, (1998).

${ }^{5}$ D. Jiles, en “Introduction to magnetism and magnetic materials”, $2^{\text {da }}$ edición, CRC Press, (1998).

6 J. Crangle, en “Solid State Magnetism”, $1^{\text {ra }}$ edición, Springer, (1991).
}

7 A. Aharoni, en "Introduction to the Theory of Ferromagnetism", $1^{\text {ra }}$ edición, Clarandon Press, Oxford, (1998).

${ }^{8}$ A. Hernando y J. Rojo, en “Física de los materiales magnéticos”, Síntesis, (2001).

${ }^{9}$ C. Suryanarayana; Progress in Mat. Sci. 46, 1-2 (2001), pág. 1.

${ }^{10}$ L.M. Socolovsky, Tesis Doctoral del Departamento de Física, Fac. Cs.Ex., Universidad Nacional de La Plata, (2002).

${ }^{11}$ Programa “WinFit”, versión 1.1, A. Rühm, (2000).

${ }^{12}$ Programa “Powder Cell”, versión 2.4 para Windows, W. Graus y G. Nolze, (2000).

${ }^{13}$ P. Scherrer; Nachr. Ges. Wiss. Gottingen 26, (1918), pág 98.

${ }^{14}$ G.K. Williamson y W.H. Hall, Acta Metall. 1, 1, (1953), pág. 22.

15 D.C. Koeningsberger y R. Prins, en " $X$-ray Absorption: principles, applications and techniques of EXAFS, SEXAFS and XANES”, Wiley, (1988).

${ }^{16}$ Programa “IFEFFIT”, version 1.2.9, Matt Newville, Univ. de Chicago, (2006).

${ }^{17}$ C. Kittel, en “Introduction to Solid State Physics”, 6 ${ }^{\text {ta }}$ edición, J. Wiley \& Sons, New York, (1986), pág. 122.

${ }^{18}$ R.E. Vandenberghe, E. De Grave y P.M.A. de Bakker; Hyp. Int. 83, (1994), pág. 29.

${ }^{19}$ Y. Endoh y Y. Ishikawa; J. Phys. Soc. Jpn. 30, (1971), pág. 1614.

${ }^{20}$ J. Martinez, S.M. Cotes y J. Desimoni; Phys. Stat Solidii b, 246, (2009), pág. 1366.

${ }^{21}$ M.B. Stearns y Y. Cheng; J. Appl. Phys. 75, (1994), pág. 6894. 
${ }^{22}$ D. Jiles, en “Introduction to magnetism and magnetic materials", $2^{\mathrm{da}}$ edición, CRC Press, (1998), pág. 105.

${ }^{23}$ N.J.O. Silva, V.S. Amaral y L.D. Carlos; Phys. Rev. B 71, (2005), pág. 184408-1. 


\section{Capítulo 5. Resultados y discusión}

En este capítulo se presentarán y discutirán los resultados obtenidos en el sistema FeMnCu. El capítulo se divide básicamente en dos grandes secciones. En la primera se presentan resultados sobre el estudio de la influencia del tiempo de molienda $\left(t_{m}\right)$ sobre los parámetros estructurales e hiperfinos de las aleaciones. En la segunda se analiza la evolución de los parámetros estructurales, hiperfinos y magnéticos con la concentración de $\mathrm{Cu}$ en muestras molidas durante $15 \mathrm{~h}$. Las técnicas experimentales empleadas para caracterizar las muestras son: Difracción de Rayos-X (DRX), Espectroscopía Mössbauer (EM) y diversas técnicas de caracterización magnética como son medias de susceptibilidad AC en función de la temperatura, para distintas frecuencias del campo alterno y en presencia de campos estáticos, ciclos de histéresis a diversas temperaturas y medidas de magnetización en función de la temperatura enfriando con y sin campo magnético.

\subsection{Materiales precursores}

Para facilitar el análisis del sistema $\mathrm{FeMnCu}$, primero se realizó el estudio del comportamiento estructural y magnético de los materiales precursores. En esta sección se presentan los resultados obtenidos por DRX y, posteriormente, los resultados magnéticos.

La figura 5.1 muestra el difractograma correspondiente a una muestra de Fe metálico molida durante 15 minutos para obtener Fe en polvo. Como se puede observar en la figura, las líneas de difracción corresponden a una estructura cúbica centrada en el cuerpo $(b c c),{ }^{1}$ con un parámetro de red de $2.865_{1} \AA$, el cual está en buen acuerdo con la bibliografía. ${ }^{1}$ El tamaño de cristalita determinado empleando la fórmula de Sherrer ${ }^{2}$ es de $30_{2} \mathrm{~nm}$. 


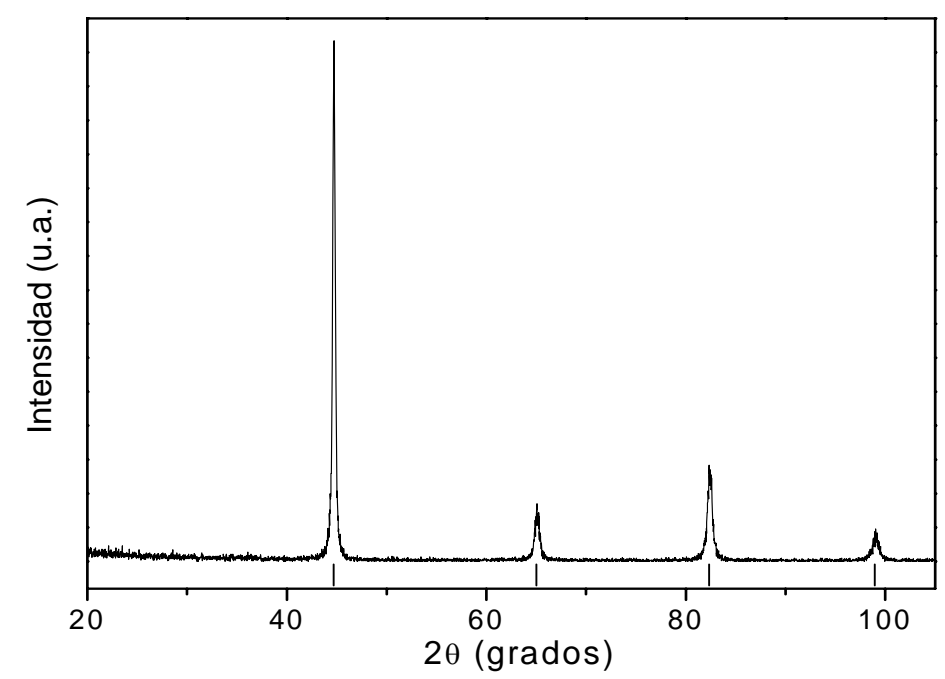

Figura 5.1: Difractograma de Fe metálico molido durante $15 \mathrm{~min}$. Las marcas al pie (| ) de la figura indican la posición de las líneas de la fase $b c c .{ }^{1}$

El difractograma correspondiente a la muestra de Mn molido durante 15 min es presentado en la figura 5.2 junto con la posición reportada para las líneas del mismo. ${ }^{3}$ La estructura de la fase $\alpha$-Mn es cúbica y el parámetro de red determinado es de $8.914_{1} \AA$ en acuerdo con lo informado, ${ }^{3} \mathrm{y}$ el tamaño de cristalita es de $50_{2} \mathrm{~nm}$.

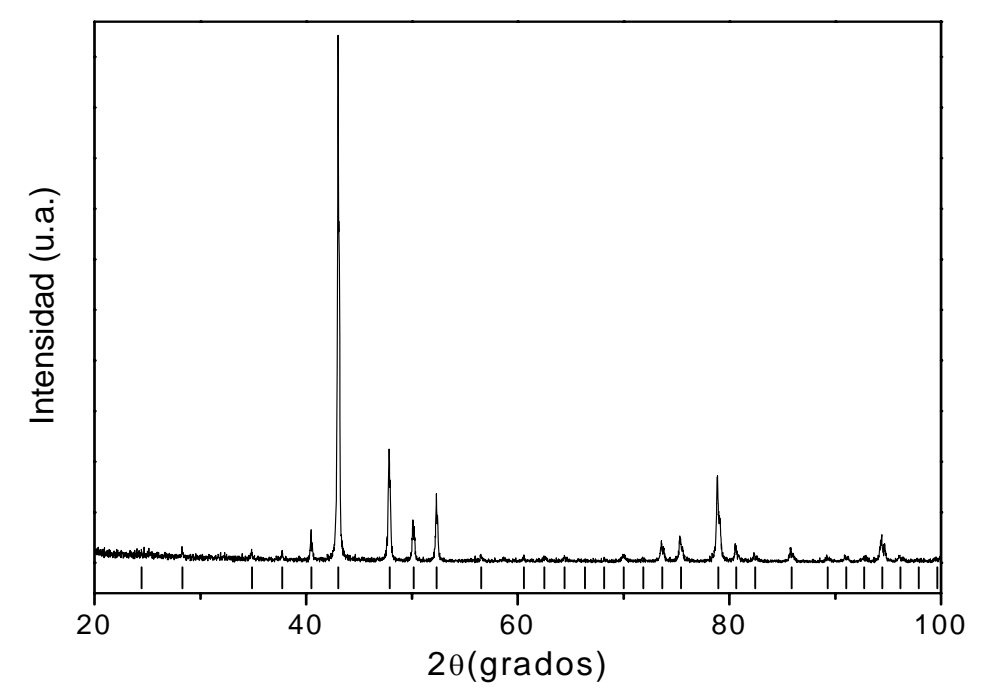

Figura 5.2: Difractograma de Mn metálico molido durante $15 \mathrm{~min}$. Las marcas al pie (| ) de la figura indican la posición de las líneas de la fase $\alpha-\mathrm{Mn}^{3}$

Por último el difractograma de la muestra de $\mathrm{Cu}$ es presentado en la figura 5.3 junto con las posiciones esperadas ${ }^{4}$ para las reflexiones de la estructura $f c c-\mathrm{Cu}$ con un parámetro de red de 
$3.615_{1} \AA$ en perfecto acuerdo con el encontrado en la bibliografía ${ }^{4}$ para las reflexiones. El tamaño medio de cristalita estimado es de $28_{2} \mathrm{~nm}$.

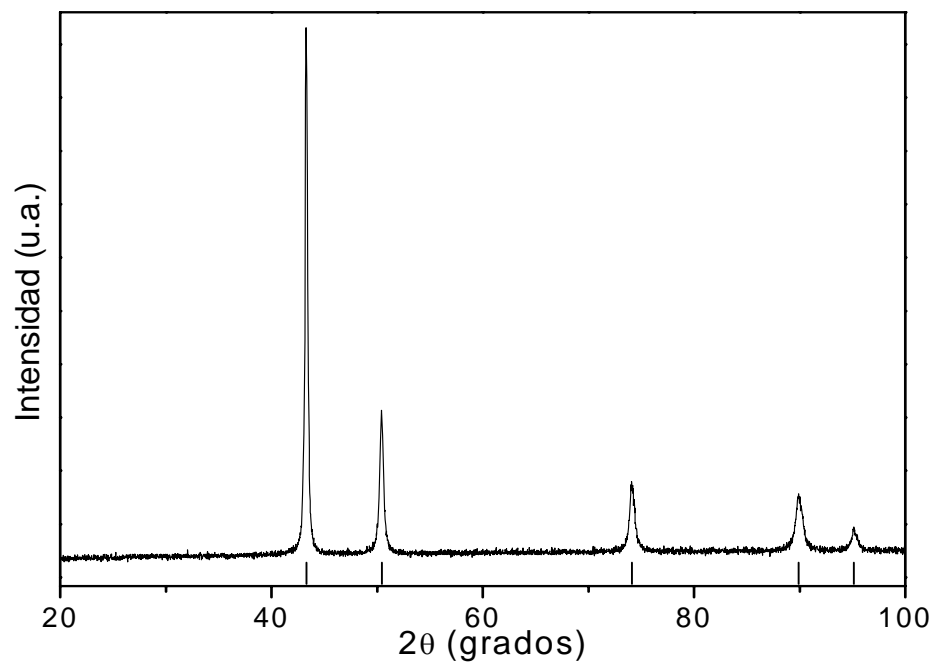

Figura 5.3: Difractograma de Cu metálico molido durante 15 min. Las marcas al pie ( | ) de la figura indican la posición de las líneas esperada según la bibliografía. ${ }^{4}$

A continuación se presentan los resultados de los ciclos se histéresis para los materiales precursores. Para este estudio los metales Fe y Mn fueron molidos durante $15 \mathrm{~h}$ en las mismas condiciones que se detallan en el Capítulo 3. Para el ciclo del Cu se empleó el material sin molerlo debido a que la gran ductibilidad. Las figuras 5.4 a 5.6 se muestran los ciclos de histéresis obtenidos para el Fe, $\mathrm{Mn}$ y $\mathrm{Cu}$, respectivamente.

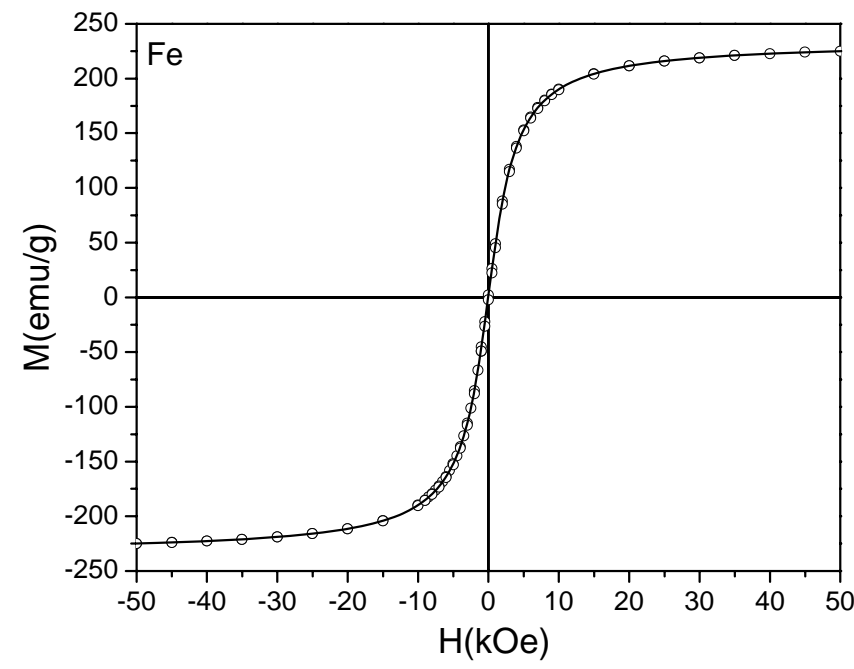

Figura 5.4: Ciclo de histéresis del Fe molido 15 h. La línea sólida es el resultado del ajuste. 


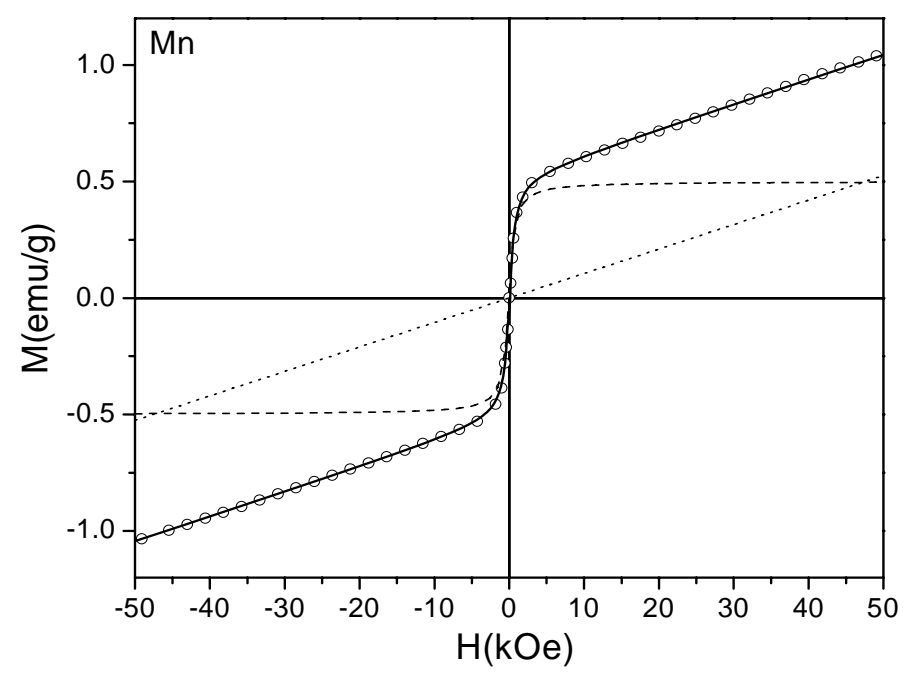

Figura 5.5: Ciclo de histéresis del Mn molido $15 \mathrm{~h}$. La línea a trozos representa la contribución ferromagnética mientras la línea punteada representa la contribución paramagnética.

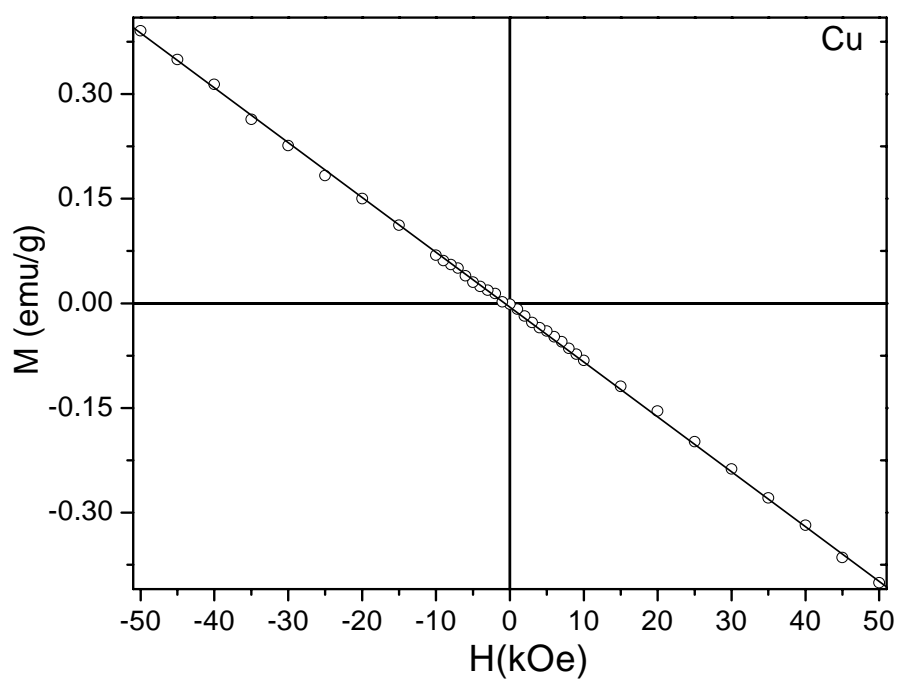

Figura 5.6: Ciclo de histéresis del Cu sin moler. La línea sólida es el resultado del ajuste.

El ciclo de histéresis de la muestra de Fe (figura 5.4) presenta el comportamiento clásico de un material ferromagnético. Para la reproducción del mismo se empleó la expresión 4.7 obteniendo como resultado un valor de la magnetización de saturación de $230_{2} \mathrm{emu} / \mathrm{g}$, la cual es alcanzada para un campo magnético de aproximadamente $1 \mathrm{~T}$. Este valor está en buen acuerdo con el obtenido en muestras de Fe masivas, las cuales tienen una magnetización de saturación de $220 \mathrm{emu} / \mathrm{gr} .{ }^{5}$ El valor del campo coercitivo es de $20_{3}$ Oe y su magnetización de remanencia de $2.0_{1} \mathrm{emu} / \mathrm{g}$.

La muestra de $\mathrm{Mn}$, el cual es paramagnético a temperatura ambiente con una temperatura de Néel de $100 \mathrm{~K},{ }^{6}$ presenta un comportamiento que se aparta del esperado para un paramagneto puro 
(figura 5.5). Como se puede observar, la magnetización posee en la región de campos bajos (0.5 $T$ ), una curvatura en forma de " $S$ " asociada a un comportamiento ferromagnético, mientras que la región de altos campos muestra una respuesta lineal, tal y como se espera para un material paramagnético. Por estos motivos, se reprodujo el ciclo de histéresis con dos contribuciones, una ferromagnética y una contribución lineal empleando para ello la expresión que resulta de la suma de las ecuaciones 4.7 y 4.9 dadas en el Capítulo 4. La magnetización de saturación obtenida en el ajuste es $0.50_{1} \mathrm{emu} / \mathrm{g}$. El origen de esta contribución está, posiblemente, asociado a una contaminación de la muestra en el proceso de molienda ya que, el acero del que están compuestos tanto los cilindros como las esferas, posee como elemento principal al Fe. Para corroborar esta hipótesis se realizó un espectro Mössbauer de la muestra de Mn molido, en el cual se observó una mínima señal que da cuenta de la presencia de $\mathrm{Mn}(\mathrm{Fe})$. Si bien no se encontró ningún rastro de bcc$\mathrm{Fe}$, no se puede descartar que exista en concentraciones menores a la resolución de la técnica (2 $\%$ ). Sabiendo ésto, y conociendo la magnetización de saturación obtenida para el ciclo del Fe puro, es posible estimar que el porcentaje de la contaminación con $\mathrm{Fe}$ no es superior al $0.2 \%$. La segunda contribución (paramagnética) está asociada al Mn, para el cual la susceptibilidad paramagnética es igual a $1.1_{1} \times 10^{-5} \mathrm{emu} / \mathrm{gOe}$, en acuerdo con el valor reportado. ${ }^{7}$

Por último, la curva para el $\mathrm{Cu}$ (figura 5.6) muestra un comportamiento lineal decreciente con el aumento del campo aplicado típico de un material diamagnético. Se pudo estimar el valor de la susceptibilidad diamagnética del $\mathrm{Cu}$ en $-8.3_{1} \times 10^{-7} \mathrm{emu} / \mathrm{gOe}$, en buen acuerdo con los datos publicados. ${ }^{7}$ 


\subsection{Cinéticas de Molienda}

Una de las primeras tareas realizadas fue determinar el tiempo óptimo de molienda necesario para asegurar el mezclado de los precursores y minimizar la posible contaminación procedente del material de los cilindros y las esferas. Para dicho fin, se prepararon dos muestras $\left[\left(\mathrm{Fe}_{79} \mathrm{Mn}_{21}\right)_{1-\mathrm{x}} \mathrm{Cu}_{\mathrm{x}}\right.$ con $\mathrm{x}=0.15 \mathrm{y} \mathrm{x}=0.30]$, las cuales fueron molidas durante $1,3,6,9,12,15 \mathrm{y} 18 \mathrm{~h}$ con descansos de $15 \mathrm{~min}$ cada $45 \mathrm{~min}$. Es importante mencionar que las muestras con mayores tiempos de molienda no fueron obtenidas a partir de las preparaciones a tiempos inferiores, sino que cada aleación fue realizada en forma independiente. A continuación se muestra en la Tabla 5.1 la nomenclatura usada para cada muestra acorde a su concentración y al tiempo de molienda.

\begin{tabular}{|c|c|c|}
\hline Tiempo total de molienda $\left(t_{m}\right)$ & $\left(\mathrm{Fe}_{79} \mathrm{Mn}_{21}\right)_{0.85} \mathrm{Cu}_{0.15}$ & $\left(\mathrm{Fe}_{79} \mathrm{Mn}_{21}\right)_{0.70} \mathrm{Cu}_{0.30}$ \\
\hline $1 \mathrm{~h}$ & FMC15-1 & FMC30-1 \\
\hline $3 \mathrm{~h}$ & FMC15-3 & FMC30-3 \\
\hline $6 \mathrm{~h}$ & FMC15-6 & FMC30-6 \\
\hline $9 \mathrm{~h}$ & FMC15-9 & FMC30-9 \\
\hline $12 \mathrm{~h}$ & FMC15-12 & FMC30-12 \\
\hline $15 \mathrm{~h}$ & FMC15-15 & FMC30-15 \\
\hline $18 \mathrm{~h}$ & FMC15-18 & FMC30-18 \\
\hline
\end{tabular}

Tabla 5.1: Nomenclatura usada para identificar las muestras estudiadas en función del tiempo de molienda. 


\subsubsection{Difracción de rayos $\mathrm{X}$}

En la figura 5.7 se presentan los difractogramas correspondientes a las dos muestras molidas durante diferentes tiempos.
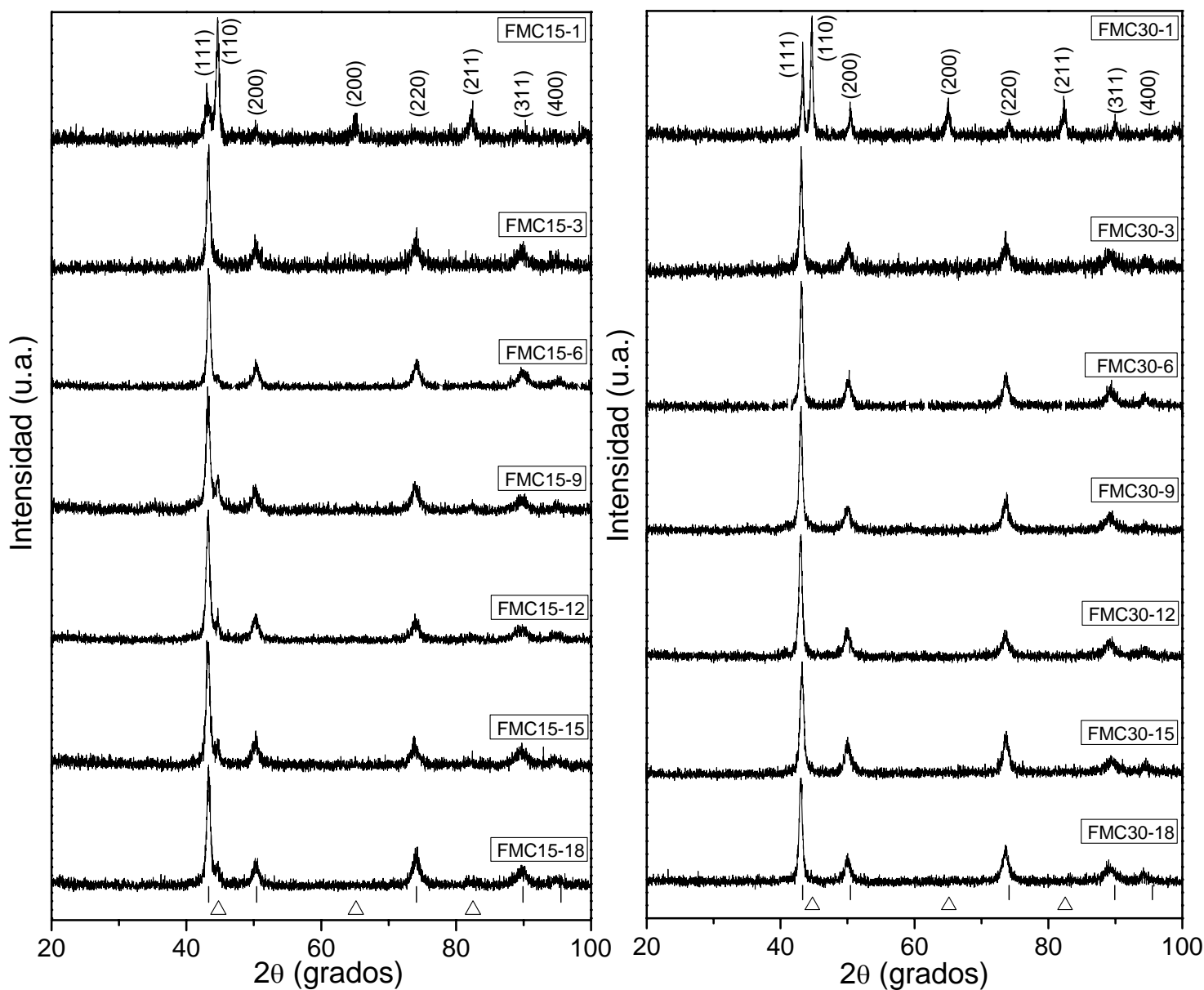

Figura 5.7: Difractogramas de las muestras con $\mathrm{x}=0.15 \mathrm{y} \mathrm{x}=0.30$ para los diferentes tiempos de molienda junto con la posición de las líneas simuladas para las fases $f c c-\mathrm{Fe}_{79} \mathrm{Mn}_{21}(\mid)$ y $b c c-\mathrm{Fe}_{90} \mathrm{Mn}_{10}(\Delta)$. En ambos difractogramas se indican los índices de Miller de ambas fases.

Los difractogramas muestran la presencia de dos fases, una con estructura $b c c$ y otra con estructura $f c c$. Para $t_{m}=1 \mathrm{~h}$ la estructura predominante es la $b c c$ para ambas concentraciones, la que se puede asociar a $b c c-\mathrm{Fe}$ o $b c c-\mathrm{Fe}(\mathrm{MnCu})$. En lo que respecta a la fase $f c c$ para $t_{m}=1 \mathrm{~h}$, ésta puede ser producto de la presencia de $f c c-\mathrm{Mn}$ o fcc-Cu no aleados, así como del comienzo de la formación de la fase ternaria $f c c-F e M n C u$. Debido a que las posiciones angulares de las reflexiones de dichas 
fases son muy próximas, junto al gran ancho de las líneas de difracción, se hace imposible determinar cuál de estas fases está siendo la responsable de los picos observados. A medida que el tiempo de molienda se incrementa, el porcentaje de estructura $b c c$ comienza a disminuir mientras que el de la fase $f c c$ aumenta. Para la muestra con $\mathrm{x}=0.15$, una pequeña fracción de la fase $b c c$ permanece para todos los tiempos de molienda, mientras que para la muestra con $\mathrm{x}=0.30$, a partir de las $6 \mathrm{~h}$ de molienda, no se observa la presencia de esta fase. Los difractogramas no presentan cambios apreciables después de $9 \mathrm{~h}$ de molienda. Estos resultados sugieren que el incremento del $t_{m}$ ayuda a la formación de la solución sólida $f c c-\mathrm{FeMnCu}$ la cual, además, es favorecida por la presencia del $\mathrm{Cu}$.

En la Tabla 5.2 y en la figura 5.8 se presenta el tamaño medio de cristalita, determinado usando la aproximación de Scherrer y considerando la línea más intensa de cada fase. Luego de una hora de molienda los tamaños de cristalita no difieren significativamente, siendo, para ambas concentraciones, de $15_{3} \mathrm{~nm}$; manteniéndose para el resto de los tiempos de molienda en valores del orden de $9_{2} \mathrm{~nm}$. El tamaño de cristalita determinado para los precursores fue de $30_{1} \mathrm{~nm}$ para el Fe, $60_{1} \mathrm{~nm}$ para el $\mathrm{Mn}$ y $28_{1} \mathrm{~nm}$ para el $\mathrm{Cu}$, esto muestra que el proceso de molienda reduce significativamente el tamaño de los cristales durante las primeras horas de trabajo mecánico.

Por otra parte, se encontró que el parámetro de red de la fase $f_{c c}\left(a_{f c c}\right)$ se incrementa con el tiempo de molienda para ambas concentraciones, alcanzando un valor constante a partir de las $12 \mathrm{~h}$ tal como se puede observar en la Tabla 5.2 y la figura 5.9. Por último, el parámetro de red tiene una dependencia con el contenido de $\mathrm{Cu}$ presente en las muestras, siendo éste más grande para concentraciones de $\mathrm{Cu}$ mayores. Después de $9 \mathrm{~h}$ de molienda el parámetro de red llega a un valor aproximadamente constante de $3.614 \AA$ y $3.625 \AA$ para $\mathrm{x}=0.15 \mathrm{y} \mathrm{x}=0.30$ respectivamente. La expansión del parámetro de red con el tiempo de molienda es atribuida a tensiones acumuladas en la red cristalina debidas a los esfuerzos inducidos por el método de fabricación. La diferencia entre el valor del parámetro de red obtenido para las diferentes concentraciones será discutida cuando se analice el comportamiento del sistema en función del contenido de $\mathrm{Cu}$.

\begin{tabular}{|c|c|c|c|c|c|}
\hline Muestra & $S_{c}(\mathrm{~nm})$ & $a_{f c c}(\AA)$ & Muestra & $S_{c}(\mathrm{~nm})$ & $a_{f c c}(\AA)$ \\
\hline FMC15-1 & $15.0_{3}$ & - & FMC30-1 & $15.4_{3}$ & $3.599_{1}$ \\
\hline FMC15-3 & $9.4_{2}$ & $3.598_{1}$ & FMC30-3 & $9.9_{2}$ & $3.608_{1}$ \\
\hline FMC15-6 & $9.3_{2}$ & $3.604_{1}$ & FMC30-6 & $9.5_{2}$ & $3.616_{1}$ \\
\hline FMC15-9 & $8.6_{2}$ & $3.610_{1}$ & FMC30-9 & $9.4_{2}$ & $3.621_{1}$ \\
\hline FMC15-12 & $8.7_{2}$ & $3.613_{1}$ & FMC30-12 & $9.1_{2}$ & $3.624_{1}$ \\
\hline FMC15-15 & $8.6_{2}$ & $3.614_{1}$ & FMC30-15 & $9.0_{2}$ & $3.625_{1}$ \\
\hline FMC15-18 & $8.7_{2}$ & $3.614_{1}$ & FMC30-18 & $9.0_{2}$ & $3.625_{1}$ \\
\hline
\end{tabular}

Tabla 5.2: Tamaño de cristalita $\left(S_{c}\right)$ y parámetro de red de la fase $f_{c c}\left(a_{f c c}\right)$, para las distintas muestras y tiempos de molienda. 


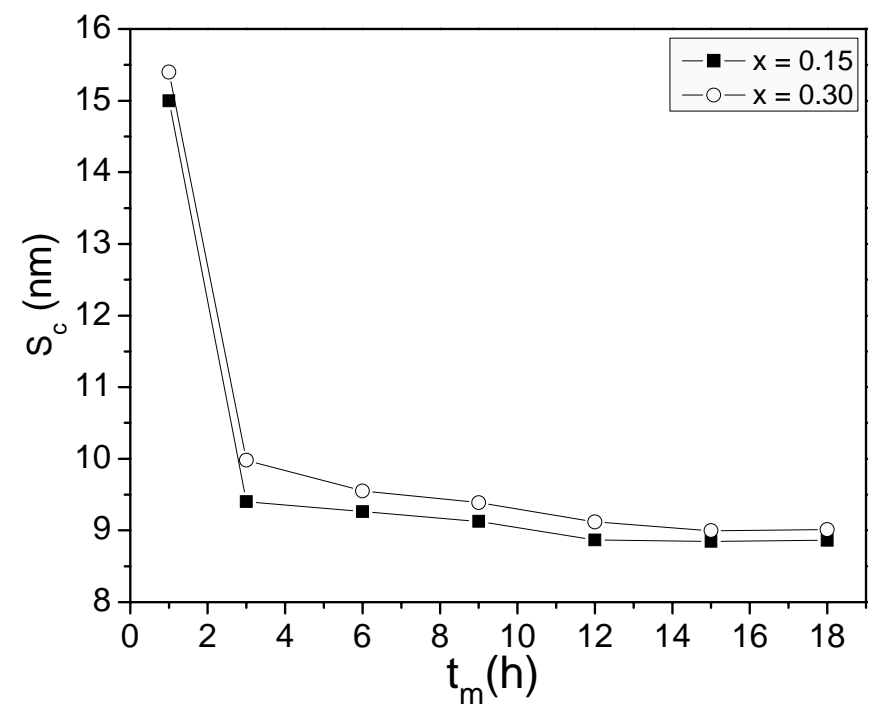

Figura 5.8: Variación del tamaño medio de cristalita con el tiempo de molienda para la muestra con $\mathrm{x}=0.15$ (cuadrados sólidos) y $\mathrm{x}=0.30$ (círculos abiertos).

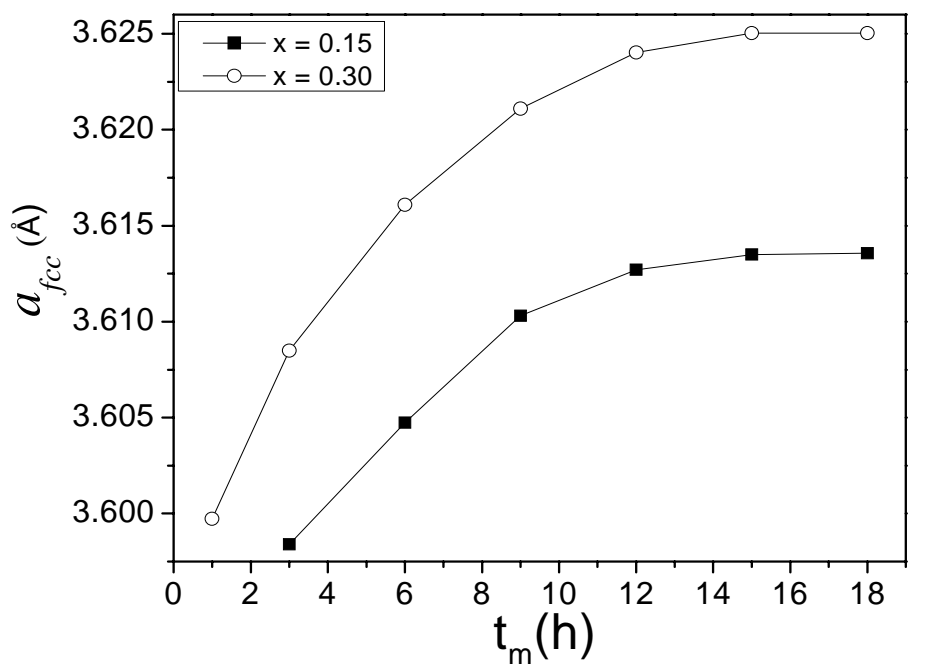

Figura 5.9: Variación del parámetro de red con el tiempo de molienda para la muestra con $\mathrm{x}=0.15$ (cuadrados sólidos) y $\mathrm{x}=0.30$ (círculos abiertos). 


\subsubsection{Espectroscopía Mössbauer}

Los espectros Mössbauer obtenidos de las muestras sometidas a trabajo mecánico diferentes tiempos se midieron en los rangos de velocidades $[-8,8 \mathrm{~mm} / \mathrm{s}]$ y $[-4,4 \mathrm{~mm} / \mathrm{s}]$. Estos espectros son presentados en las figuras 5.10 y 5.11 respectivamente. Los mismos fueron reproducidos usando dos distribuciones de campos magnéticos hiperfinos (DCMH), una con valores del campo hiperfino de aproximadamente $31 \mathrm{~T}$, llamada de aquí en delante de alto campo, y otra con valores de aproximadamente $2 \mathrm{~T}$, la cual se llamará de aquí en más de bajo campo. Una discusión completa sobre el modelo de ajuste escogido será presentada más tarde cuando se estudien los resultados en función del contenido de $\mathrm{Cu}$ y la temperatura de medida. Los parámetros hiperfinos resultantes del proceso de ajuste se muestran en las Tablas 5.3 y 5.4. Acorde con lo observado por DRX y a resultados previos, ${ }^{8}$ la componente de alto campo es asociada a la formación de la solución sólida bcc- $\mathrm{Fe}(\mathrm{Mn}, \mathrm{Cu})$ y la de bajo campo a la solución sólida $f c c-\mathrm{FeMnCu}$. Al cabo de una hora de molienda los espectros correspondientes a ambas concentraciones presentan un sextete asociado con la fase $b c c$, siendo su fracción relativa al total del espectro de un $88 \%$ y un $74 \%$ para las muestras con $\mathrm{x}=0.15 \mathrm{y} \mathrm{x}=0.30$, respectivamente. Para los tiempos de molienda más largos el porcentaje de esta fase decrece rápidamente, alcanzando valores menores que el $10 \%$ para la muestra con $\mathrm{x}=0.15$, mientras que en la muestra con $\mathrm{x}=0.30$ no es detectable. Para $\mathrm{x}=0.15$, a partir de las $12 \mathrm{~h}$ de molienda, la fracción de bcc permanece constante, en acuerdo con lo observado en los resultados de DRX. 

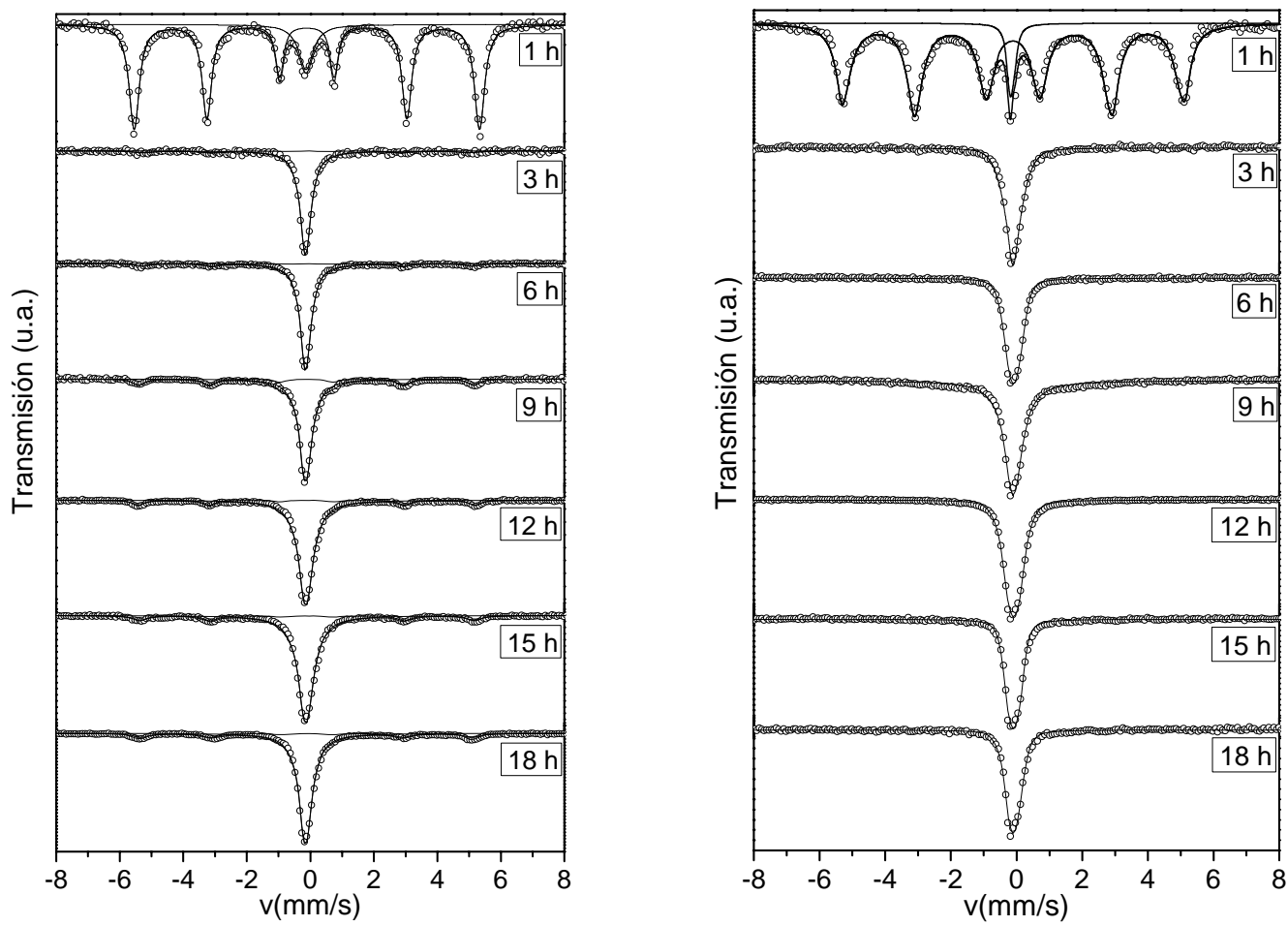

Figura 5.10: Espectros Mössbauer adquiridos en el rango mayor de velocidades para la muestra con $\mathrm{x}=0.15$ (izq.) y $\mathrm{x}=0.30$ (der.) para los distintos tiempos de molienda. Las líneas sólidas corresponden al resultado de los ajustes de los espectros.
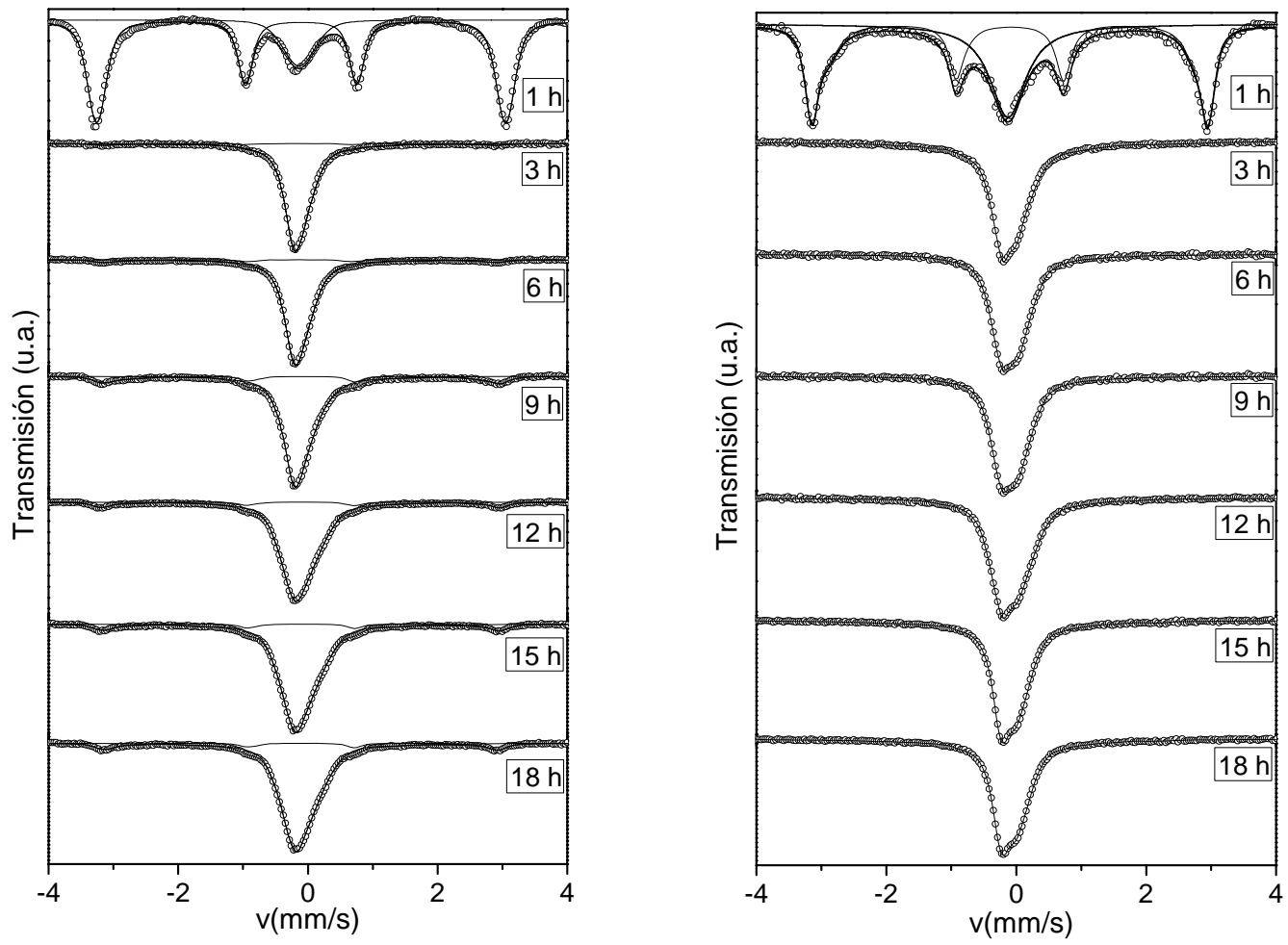

Figura 5.11: Espectros Mössbauer adquiridos en el rango menor de velocidades de las muestras con $\mathrm{x}=0.15$ (izq.) y $\mathrm{x}=0.30$ (der.) para los distintos tiempos de molienda. Las líneas sólidas corresponden al resultado del ajuste de los espectros. 


\begin{tabular}{|c|c|c|c|c|c|c|c|c|}
\cline { 2 - 10 } \multicolumn{1}{c|}{} & \multicolumn{4}{c|}{ Interacción de alto campo } & \multicolumn{4}{c|}{ Interacción de bajo campo } \\
\hline Muestra & $\mathrm{B}_{\mathrm{hf}}(\mathrm{T})$ & $\sigma(\mathrm{T})$ & $\delta(\mathrm{mm} / \mathrm{s})$ & $\mathrm{f}$ & $\mathrm{B}_{\mathrm{hf}}(\mathrm{T})$ & $\sigma(\mathrm{T})$ & $\delta(\mathrm{mm} / \mathrm{s})$ & $\mathrm{f}$ \\
\hline FMC15-1 & 32.3 & 7.1 & 0.00 & 0.88 & - & - & - & 0.12 \\
\hline FMC15-3 & - & - & - & 0.00 & 1.02 & 0.8 & -0.03 & - \\
\hline FMC15-6 & 31.4 & 7.3 & -0.01 & 0.04 & 1.05 & 0.8 & -0.03 & 0.96 \\
\hline FMC15-9 & 31.2 & 7.3 & -0.01 & 0.07 & 1.22 & 0.9 & -0.02 & 0.93 \\
\hline FMC15-12 & 31.1 & 7.3 & -0.01 & 0.05 & 1.40 & 1.0 & -0.01 & 0.95 \\
\hline FMC15-15 & 31.1 & 7.3 & -0.01 & 0.05 & 1.41 & 1.0 & -0.01 & 0.95 \\
\hline FMC15-18 & 31.1 & 7.3 & -0.01 & 0.05 & 1.41 & 1.0 & -0.01 & 0.95 \\
\hline
\end{tabular}

Tabla 5.3: Resultados de los ajustes para el campo magnético hiperfino medio $\left(\mathrm{B}_{\mathrm{hf}}\right)$, momento de $2^{\text {do }}$ orden $(\sigma)$, corrimiento isomérico medio $(\delta)$ y fracción relativa al total del espectro para la interacción de alto y bajo campo (f) en las muestras con $\mathrm{x}=0.15$.

\begin{tabular}{|c|c|c|c|c|c|c|c|c|}
\cline { 2 - 10 } \multicolumn{1}{c|}{} & \multicolumn{4}{c|}{ Interacción de alto campo } & \multicolumn{4}{c|}{ Interacción de bajo campo } \\
\hline Muestra & $\mathrm{B}_{\mathrm{hf}}(\mathrm{T})$ & $\sigma(\mathrm{T})$ & $\delta(\mathrm{mm} / \mathrm{s})$ & $\mathrm{f}$ & $\mathrm{B}_{\mathrm{hf}}(\mathrm{T})$ & $\sigma(\mathrm{T})$ & $\delta(\mathrm{mm} / \mathrm{s})$ & $\mathrm{f}$ \\
\hline FMC30-1 & 32.0 & 7.5 & 0.00 & 0.74 & - & - & - & 0.26 \\
\hline FMC30-3 & - & - & - & 0.00 & 1.12 & 0.7 & 0.02 & 1.00 \\
\hline FMC30-6 & - & - & - & 0.00 & 1.14 & 0.7 & 0.02 & 1.00 \\
\hline FMC30-9 & - & - & - & 0.00 & 1.21 & 0.7 & 0.03 & 1.00 \\
\hline FMC30-12 & - & - & - & 0.00 & 1.33 & 0.8 & 0.03 & 1.00 \\
\hline FMC30-15 & - & - & - & 0.00 & 1.32 & 0.8 & 0.03 & 1.00 \\
\hline FMC30-18 & - & - & - & 0.00 & 1.32 & 0.8 & 0.03 & 1.00 \\
\hline
\end{tabular}

Tabla 5.4: Resultados de los ajustes para el campo magnético hiperfino medio $\left(\mathrm{B}_{\mathrm{hf}}\right)$, momento de $2^{\mathrm{do}}$ orden $(\sigma)$, corrimiento isomérico medio $(\delta)$ y fracción relativa al total del espectro para la interacción de alto y bajo campo (f) en las muestras con $\mathrm{x}=0.30$.

Para ambas concentraciones el campo hiperfino medio $\left(\mathrm{B}_{\mathrm{hf}}\right)$ y el corrimiento isomérico medio $(\delta)$ de la componente de alto campo para $\mathrm{t}_{\mathrm{m}}=1 \mathrm{~h}$, presentan valores cercanos a los correspondientes al $b c c-\mathrm{Fe}\left(B_{h f}=33 \mathrm{~T}\right.$ y $\left.\delta=0.00 \mathrm{~mm} / \mathrm{s}\right) .{ }^{9}$ Esto muestra que para el tiempo de molienda más corto, ambas muestras están formadas principalmente por $b c c-\mathrm{Fe}(\mathrm{Mn}, \mathrm{Cu})$. Para los tiempos de molienda sucesivos esta interacción sólo puede ser detectada en la muestra con $\mathrm{x}=$ 0.15 , obteniendo valores del $B_{h f}$ levemente superiores a $31 \mathrm{~T}$ con un $\delta$ de $-0.01 \mathrm{~mm} / \mathrm{s}$ y con una fracción relativa al total del espectro de un $5 \%$. La distribución de probabilidades de esta interacción es mostrada en la figura 5.12. 


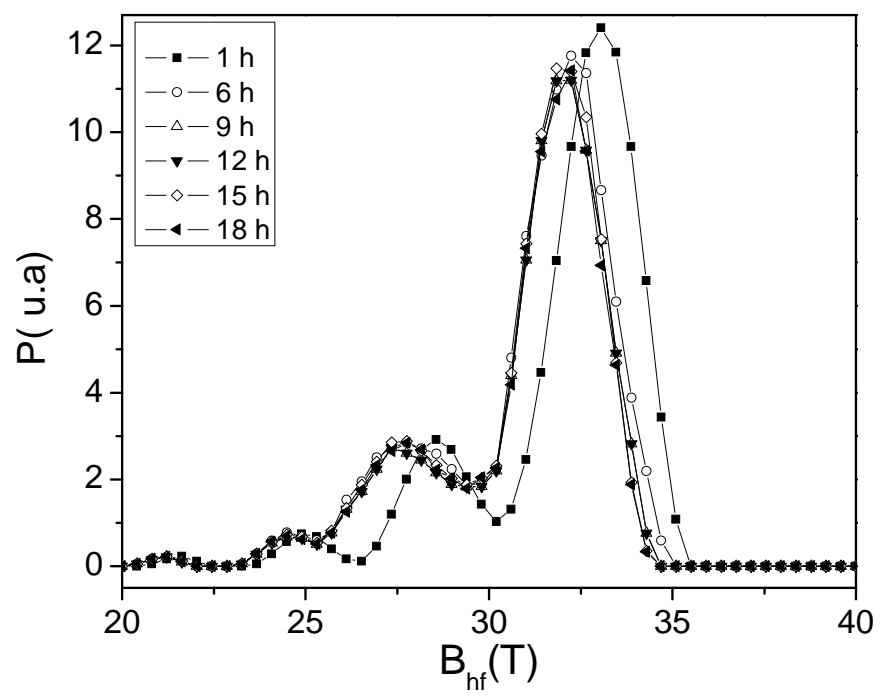

Figura 5.12: Distribución de probabilidades de la interacción de alto campo de las muestras con $\mathrm{x}=0.15$.

Allí se observan varios máximos relativos, los cuales pueden ser asociados con los diferentes entornos atómicos del Fe en la estructura $b c c .{ }^{10} \mathrm{~A}$ partir de $9 \mathrm{~h}$ de molienda no se aprecian cambios en la forma de la distribución, indicando que la fase bcc ha llegado a un estado estacionario.

La distribución de probabilidades para la componente de bajo campo es presentada en la figura 5.13. Para ambas concentraciones, el máximo de la distribución se corre hacia valores más altos del campo magnético hiperfino a medida que se incrementa el tiempo de molienda. Se puede observar de estos gráficos que existen dos regiones definidas, una para $t_{m} \leq 9 h$ y otra para $t_{m}>9 h$. A partir de las $12 \mathrm{~h}$ las distribuciones de campo hiperfino no presentan cambios indicando que el sistema ha alcanzado un estado estacionario.
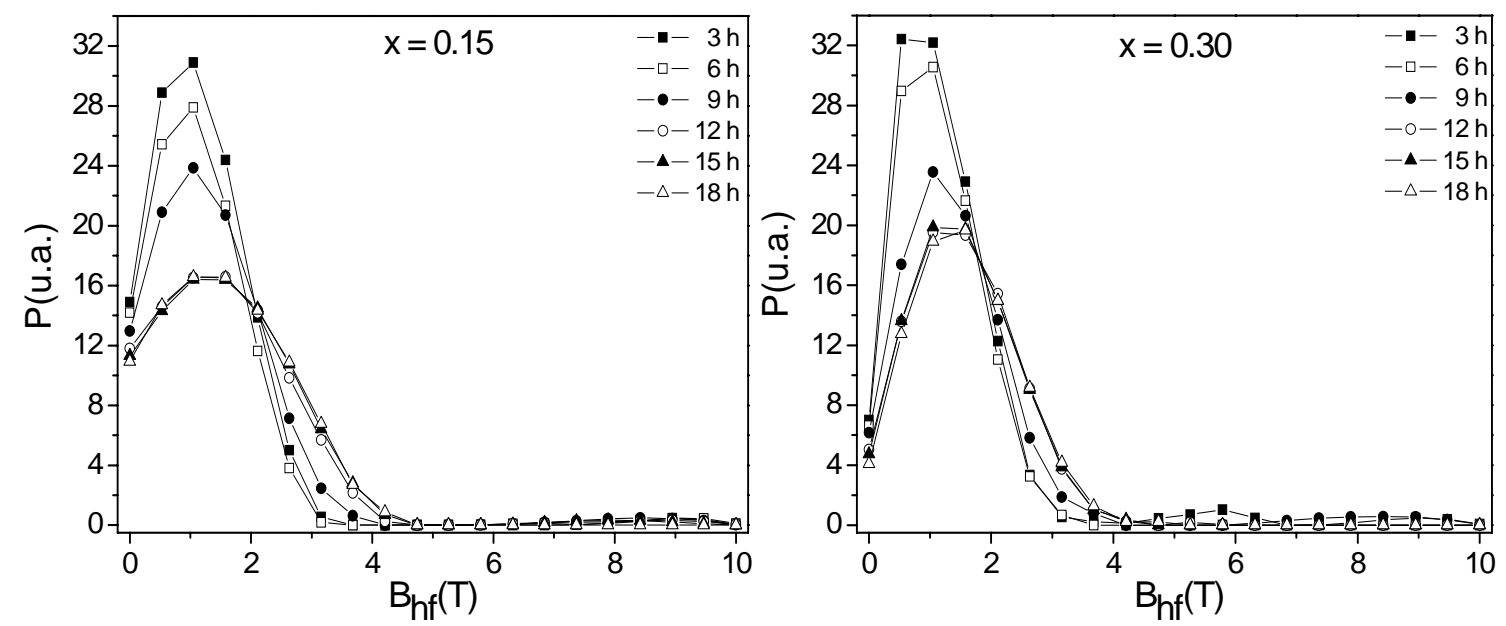

Figura 5.13: Distribución de probabilidades de la interacción de bajo campo. 
Se puede notar que existe una contribución a campo cero en las distribuciones correspondientes a ambas concentraciones, la cual va disminuyendo a medida que se aumenta el tiempo de molienda. Una posible explicación a esto podría ser la presencia de desorden en los átomos de la superficie de las partículas, las cuales podrían presentarse en un estado paramagnético $o$, al ser partículas de un tamaño lo suficientemente pequeño, exhibir un comportamiento superparamagnético. Si bien estás dos hipótesis son razonables, no explicarían por qué el valor de la distribución a campo cero es distinto para la muestra con $\mathrm{x}=0.15 \mathrm{y} \mathrm{x}=0.30$, ya que para ambas muestras el tamaño de cristalita determinado es similar. Si se piensa solamente en la disminución de la componente a campo cero con el tiempo de molienda, se puede hallar una explicación al comportamiento observado pensando que para tiempos cortos las muestras no han alcanzado una mezcla total de sus elementos. Según las referencias $[11,12]$ la temperatura de Néel de la fase fccFeMn depende de la concentración de Mn, siendo más baja a menor contenido de Mn. Esta temperatura es inferior a RT para una concentración de Mn de aproximadamente $14 \%$ at., por lo que es posible asociar la contribución a campo cero observada para los primeros tiempos de molienda con una fracción de la muestra que se encuentra en estado paramagnético al no haberse alcanzado la mezcla total de los elementos. Al ir aumentando el tiempo se comenzarán a integrar los constituyentes y así la fracción de la muestra en estado paramagnético disminuirá.

La figura 5.14 muestra el comportamiento del campo magnético hiperfino promedio $\left(\mathrm{B}_{\mathrm{hf}}\right)$ y el corrimiento isomérico promedio $(\delta)$ en función del tiempo de molienda. En ambas muestras se observa un incremento en los valores de estos parámetros a medida que el tiempo de molienda crece. También de aquí se puede ver que para $t_{\mathrm{m}} \geq 12 \mathrm{~h}$ los parámetros hiperfinos no presentan cambios significativos, lo cual indica que la mezcla atómica de los elementos ha alcanzado un estado estacionario. Los distintos valores obtenidos para $\mathrm{B}_{\mathrm{hf}} \mathrm{y} \delta$ en el estado estacionario, producto de la diferencia en el contenido de $\mathrm{Cu}$ de las preparaciones serán discutidos posteriormente en el marco de los resultados obtenidos para las muestras $0.00 \leq \mathrm{x} \leq 0.30$. 

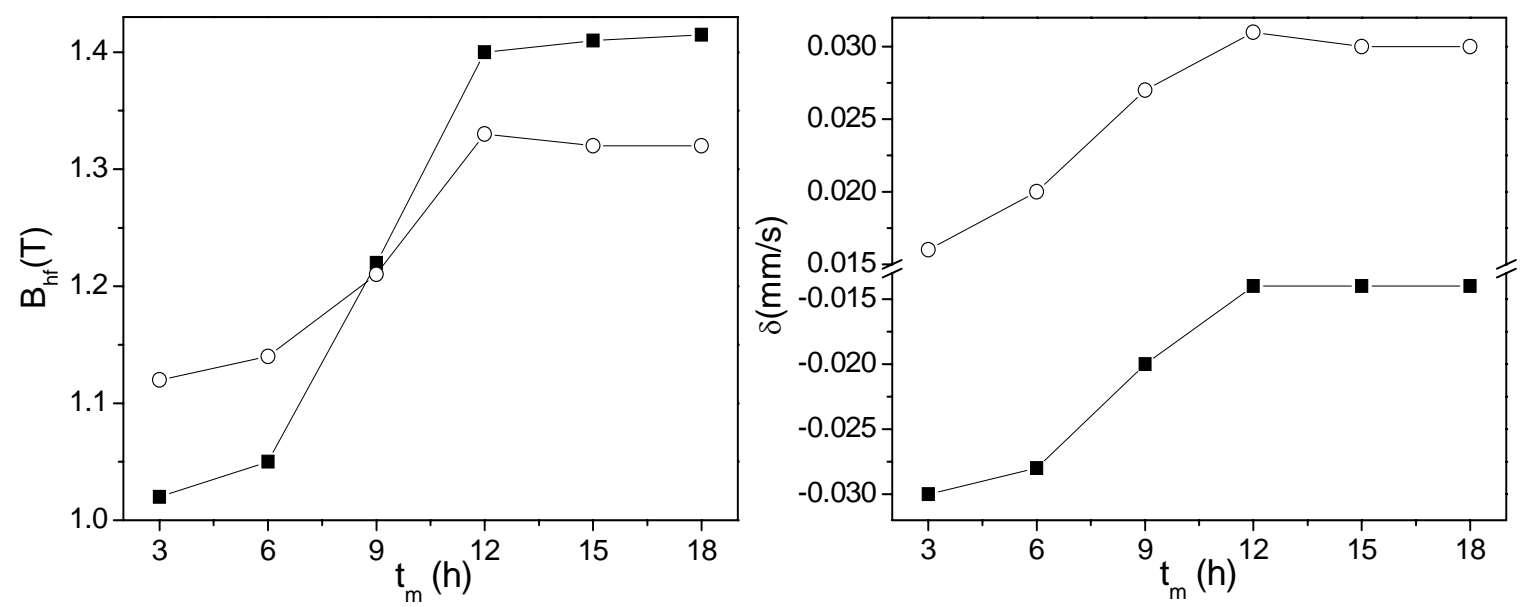

Figura 5.14: Campo magnético hiperfino (izq.) y corrimiento isomérico (der.) en función del tiempo de molienda para la componente de campo bajo. En ambas figuras los cuadrados sólidos y los círculos abiertos corresponden a $\mathrm{x}=0.15 \mathrm{y} \mathrm{x}=0.30$, respectivamente.

Luego del estudio de la cinética de molienda, y a la luz de los resultados obtenidos, se concluye que $t_{m}=15 \mathrm{~h}$ totales es apropiado para alcanzar el estado estacionario de la aleación. Si bien a las $12 \mathrm{~h}$ de molienda ya no se aprecian cambios, se escogió un tiempo mayor para asegurar que la mezcla de los precursores haya alcanzado su estado final, considerando que esto no aumentará significativamente la contaminación producto del proceso de molienda. 


\subsection{Evolución con el contenido de $\mathrm{Cu}$ de los parámetros estructurales, hiperfinos y magnéticos}

\subsubsection{Estudio estructural}

\section{Fases presentes y tamaños de partícula}

A continuación se presentan los resultados de DRX para las muestras $\left(\mathrm{Fe}_{79} \mathrm{Mn}_{21}\right)_{1-\mathrm{x}} \mathrm{Cu}_{\mathrm{x}}$ con 0 $\leq \mathrm{x} \leq 30$. Los difractogramas de la figura 5.15 muestran líneas anchas teniendo en todos los casos la fase mayoritaria una estructura $f c c$. Debido al pequeño tamaño de cristalita de las aleaciones producto del método de fabricación se pueden observar líneas de difracción anchas. ${ }^{13}$ Para las muestras con $\mathrm{x}=0.00, \mathrm{x}=0.10 \mathrm{y} \mathrm{x}=0.15$ la línea principal de la fase $f c c\left(2 \theta \approx 43^{\circ}\right)$ tiene una asimetría en su flanco derecho. Ésta es asignada a la reflexión más intensa (110) de la fase de estructura $b c c$ proveniente de $\mathrm{Fe}$ o $\mathrm{Fe}(\mathrm{MnCu})$, pudiéndose observar que el incremento de $\mathrm{Cu}$ favorece la formación de la fase $f c c$. En la Tabla 5.5 se muestra la evolución del parámetro de red con la concentración de $\mathrm{Cu}$. Se nota que existe un incremento en $a_{f c c}$ desde $3.602_{1}$ a $3.625_{1} \AA$ para $\mathrm{x}=0.00$ y 0.30 , respectivamente. 


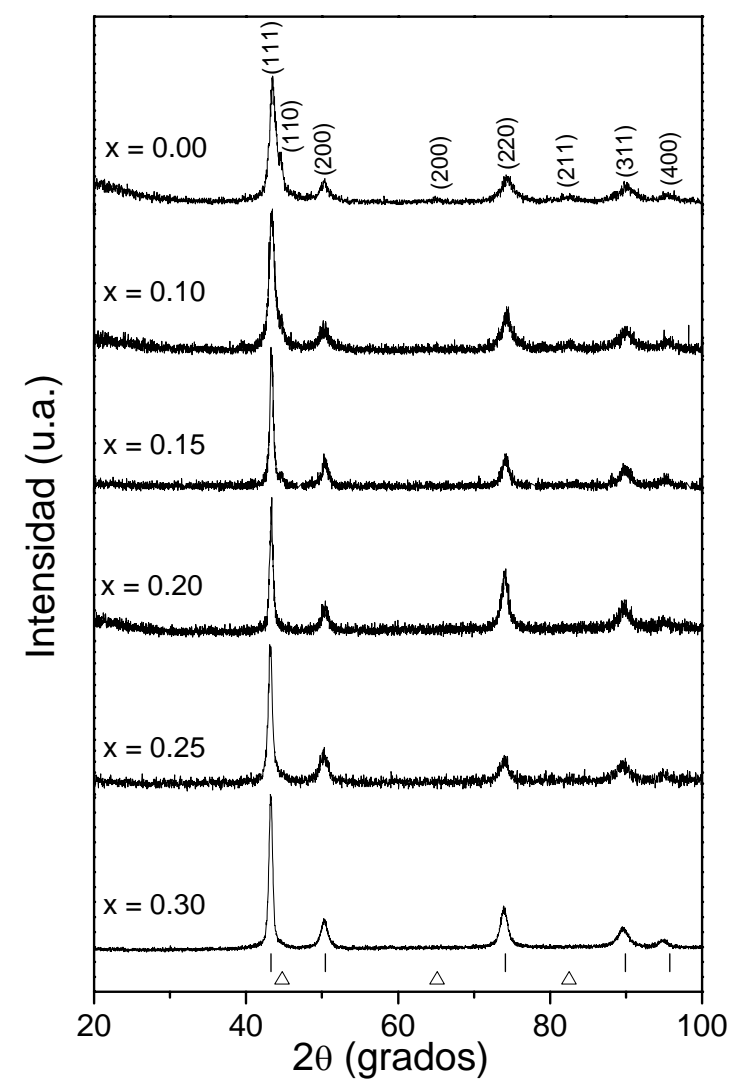

Figura 5.15: Difractogramas de las muestras para las concentraciones de $\mathrm{Cu}$ junto con la posición de las líneas simuladas para las fases $f c c-\mathrm{Fe}_{79} \mathrm{Mn}_{21}(\mid)$ y $b c c-\mathrm{Fe}_{90} \mathrm{Mn}_{10}(\Delta)$.

\begin{tabular}{|c|c|c|}
\hline $\mathrm{x}$ & $a_{f c c}(\AA)$ & $S_{c}(\mathrm{~nm})$ \\
\hline 0.00 & $3.602_{1}$ & $8.2_{2}$ \\
\hline 0.10 & $3.610_{1}$ & $8.9_{2}$ \\
\hline 0.15 & $3.614_{1}$ & $9.3_{2}$ \\
\hline 0.20 & $3.618_{1}$ & $8.7_{2}$ \\
\hline 0.25 & $3.622_{1}$ & $9.2_{2}$ \\
\hline 0.30 & $3.625_{1}$ & $9.8_{1}$ \\
\hline
\end{tabular}

Tabla 5.5: Evolución del tamaño de cristalita $\left(S_{c}\right)$, del parámetro de red de la fase $f c c\left(a_{f c c}\right)$ en función del contenido de $\mathrm{Cu}$.

En la figura 5.16 se muestra la evolución del parámetro de red correspondiente a la estructura $f c c$ con el contenido de $\mathrm{Cu}$. Una de las primeras características que se puede observar es que el valor de $a_{f c c}$, para la muestra $\sin \mathrm{Cu}(\mathrm{x}=0.00)$ es mayor al encontrado en muestras con la misma concentración $\left(\mathrm{Fe}_{79} \mathrm{Mn}_{21}\right)$ preparadas por métodos convencionales. ${ }^{14}$ Este incremento es de $0.01 \AA$ cuando se compara el valor obtenido en esta Tesis $\left(3.602_{1} \AA\right)$ con el reportado en la bibliografía para una muestra preparada en horno de arco $\left(3.5962_{2} \AA\right) .{ }^{14}$ Un efecto similar fue 
observado previamente en los sistemas $\mathrm{FeMn},{ }^{15} \mathrm{FeNi},{ }^{16} \mathrm{y} \mathrm{FeCu}^{17}$ preparados por aleado mecánico, atribuyendo este efecto al proceso de molienda en sí mismo. Se observa que el aumento en la cantidad de $\mathrm{Cu}$ en la aleación induce un incremento en el parámetro de red a razón de $7.8_{2} \times 10^{-4}$ $\AA / \%$ at. de $\mathrm{Cu}$.. Debido a que el $\mathrm{Cu}$ posee un radio metálico mayor $(1.28 \AA)^{18}$ al del Fe $(1.26 \AA)^{18} \mathrm{y}$ al del Mn $(1.26 \AA),{ }^{18}$ la red cristalina deberá expandirse para incorporar al $\mathrm{Cu}$ como parte de ella, por lo tanto se esperará un aumento en el parámetro de red al substituir un átomo de $\mathrm{Fe}$ o Mn en la estructura por uno de $\mathrm{Cu}$. De modo que el aumento en $a_{f c c}$ puede ser atribuido a la incorporación substitucional de átomos de $\mathrm{Cu}$ en la estructura $f c c$ del FeMn

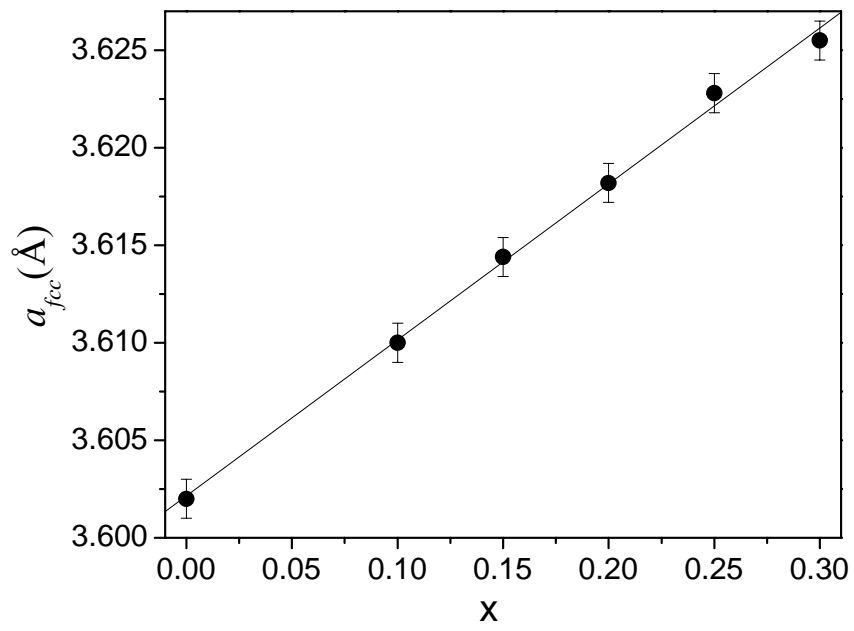

Figura 5.16: Variación del parámetro de red con el contenido de $\mathrm{Cu}$.

El tamaño medio de cristalita resulta en torno a los $9 \mathrm{~nm}$ para todas las muestras (Tabla 5.5), indicando que ni un proceso de soldadura ni uno de fractura se favorece con el agregado de $\mathrm{Cu}$. A continuación, en la figura 5.17, se muestra una fotografía realizada por microscopía electrónica de transmisión (TEM) en la muestra con $\mathrm{x}=0.20$, junto con un análisis de la distribución de tamaños de partículas obtenidos de la imagen. Los datos de los tamaños obtenidos de la imagen TEM fueron organizados en forma de histograma, al cual se lo ajustó con una distribución log-normal. El valor medio de la distribución de tamaños es de $8 \mathrm{~nm}$ con una dispersión de $5 \mathrm{~nm}$. Este valor está en muy buen acuerdo con el determinado por DRX. 

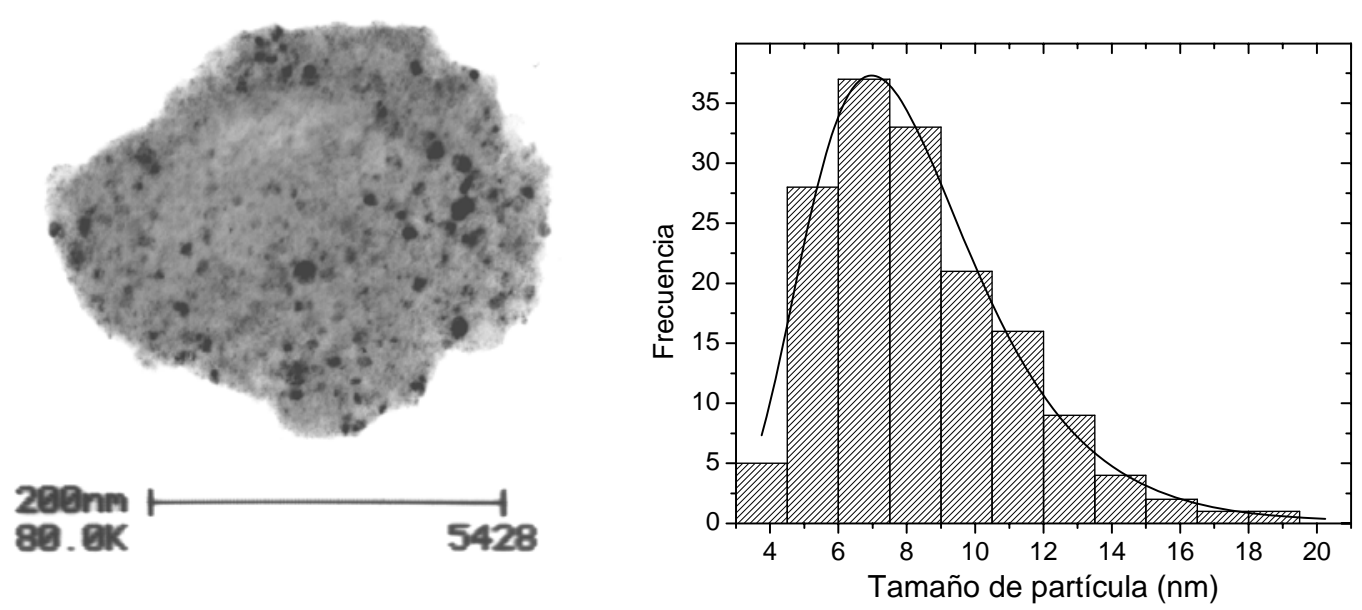

Figura 5.17: Imagen de microscopía electrónica de transmisión correspondiente a la muestra con $\mathrm{x}=0.20$ (izq.). Distribución de tamaños de partícula estimados a partir de la imagen TEM (der.), la línea sólida representa el ajuste con una distribución log-normal de los datos experimentales.

\section{Estado de oxidación y número de vecinos}

El estado de oxidación y la cantidad y clase de primeros vecinos fueron determinados por espectroscopía de absorción de rayos $\mathrm{X}$ (XAS) en el borde $K$ del Fe y en el borde $K$ del Cu. Debido a los problemas encontrados para hacer las medidas en el borde $K$ del $\mathrm{Fe}$, los cuales fueron explicados en el Capítulo 4, se presentan sólo los resultados correspondientes a la región cercana al borde de absorción (XANES) del mismo.

El estado de oxidación del $\mathrm{Fe}$ y del $\mathrm{Cu}$ fue determinado mediante el análisis de la región cercana al borde de absorción. En la figura 5.18 y 5.19 se muestran los espectros de la región XANES correspondientes a las medidas realizadas en los bordes borde $K$. 


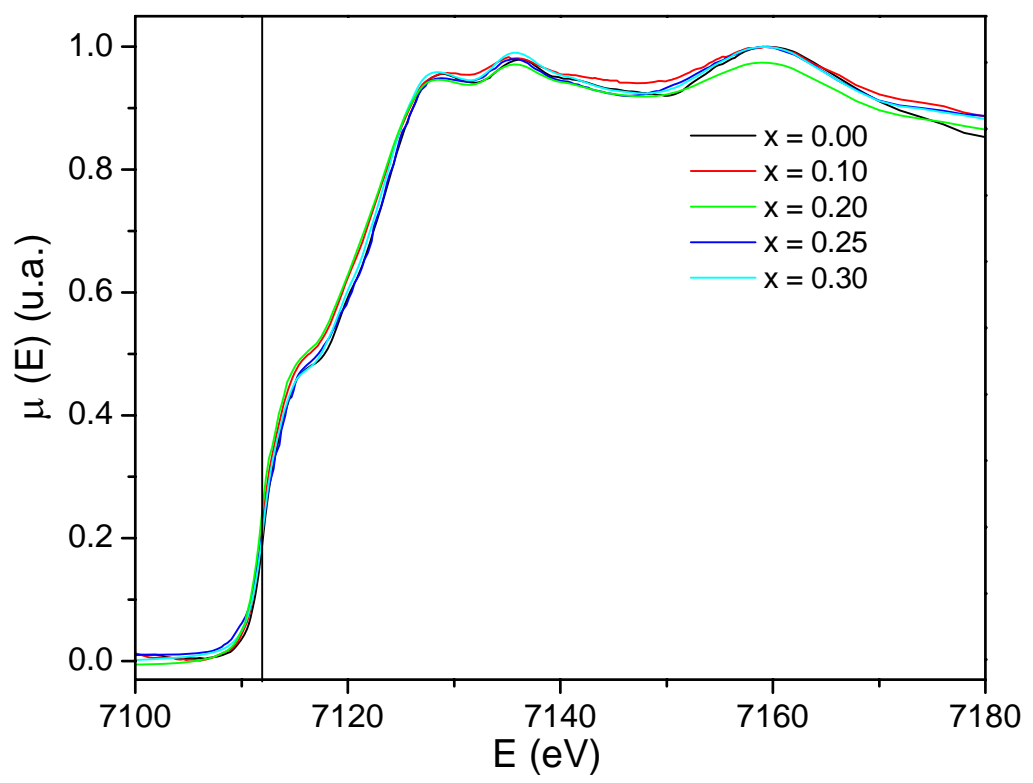

Figura 5.18: Región XANES del espectro de absorción medido en el borde $K$ del Fe.

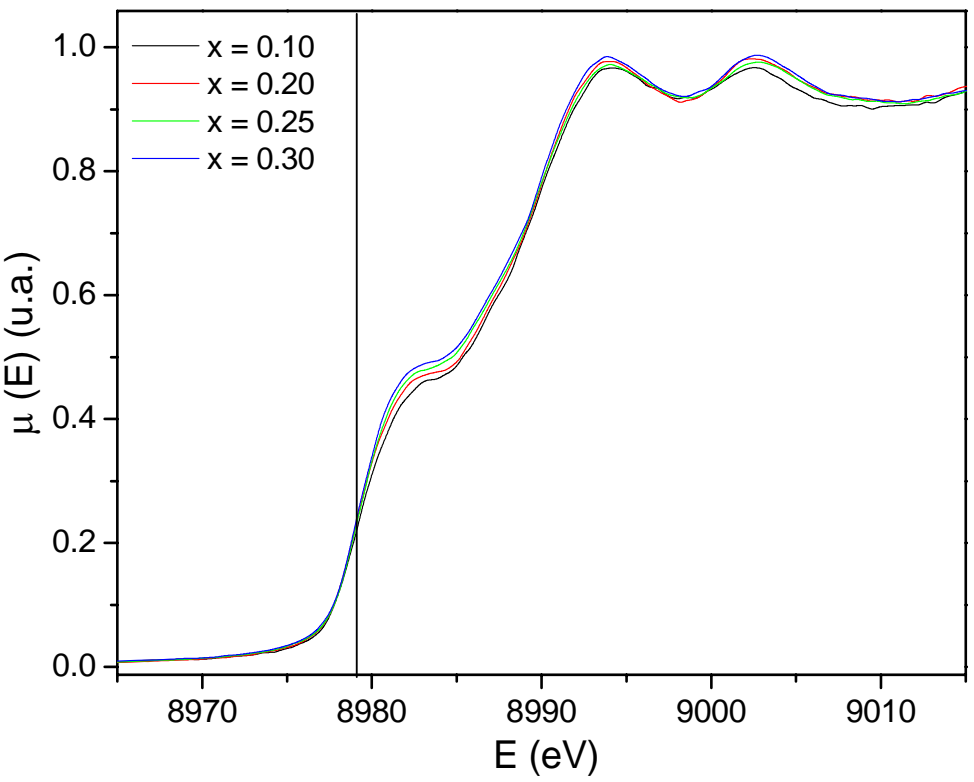

Figura 5.19: Región XANES del espectro de absorción medido en el borde $\mathrm{K}$ del $\mathrm{Cu}$.

Como ya se mencionó en el Capítulo 4 no ha sido desarrollada aún ninguna teoría para el estudio de la región XANES. Por este motivo, generalmente se recurre a un análisis cualitativo comparando espectros con los de muestras patrones. En este caso, que se quiere determinar si existe algún grado de oxidación en las muestras, se analizará la energía del borde de absorción, pues este valor es muy sensible a los estados de oxidación en las vecindades del átomo absorbente. ${ }^{19,20}$ A continuación, en la figura 5.20 se muestra, a modo de ejemplo, cómo varía la 
posición (energía) del borde $K$ en distintas muestras de óxidos de $\mathrm{Fe} .{ }^{21}$ Como se puede observar, existe una clara dependencia de la posición del borde de absorción con el estado de oxidación.

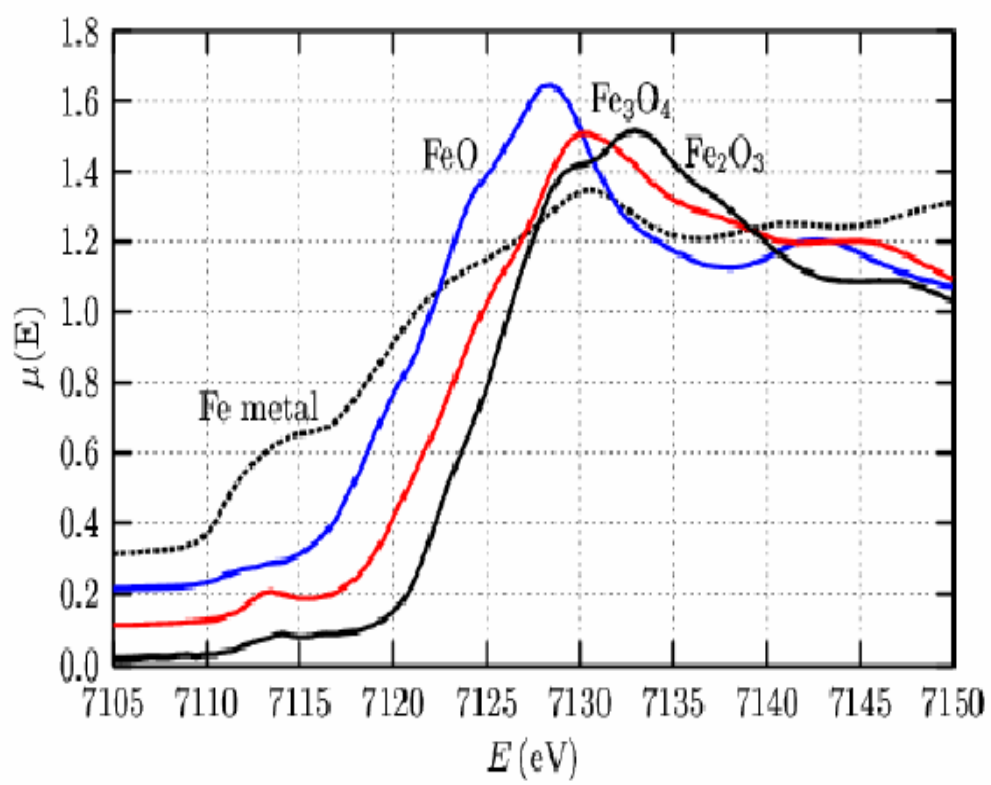

Figura 5.20: Comparación de la región XANES correspondientes a espectros del Fe metálico y óxidos de Fe.

Se presentan en la figura 5.21 y 5.22 las curvas de $d \mu / d E$ en función de $E$ para las medidas realizadas junto a las correspondientes a los patrones de $\mathrm{Fe}$ y de $\mathrm{Cu}$, para así determinar la posición del borde $K$.

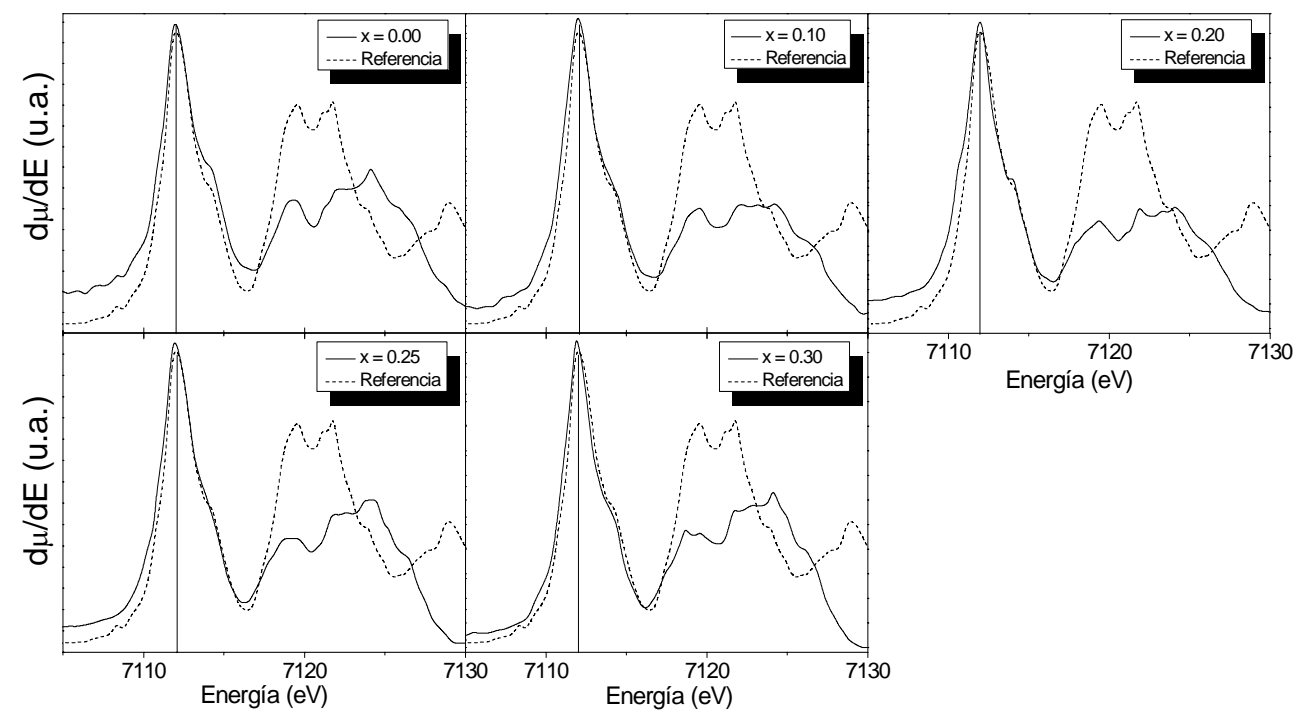

Figura 5.21: Derivada de los espectros de absorción en la región XANES (línea sólida) con las correspondientes al Fe metálico (línea a trozos). La línea vertical indica la energía del borde $K$ del $\mathrm{Fe}\left(\mathrm{E}_{\mathrm{b}}=\right.$ $7112 \mathrm{eV})^{22}$ 


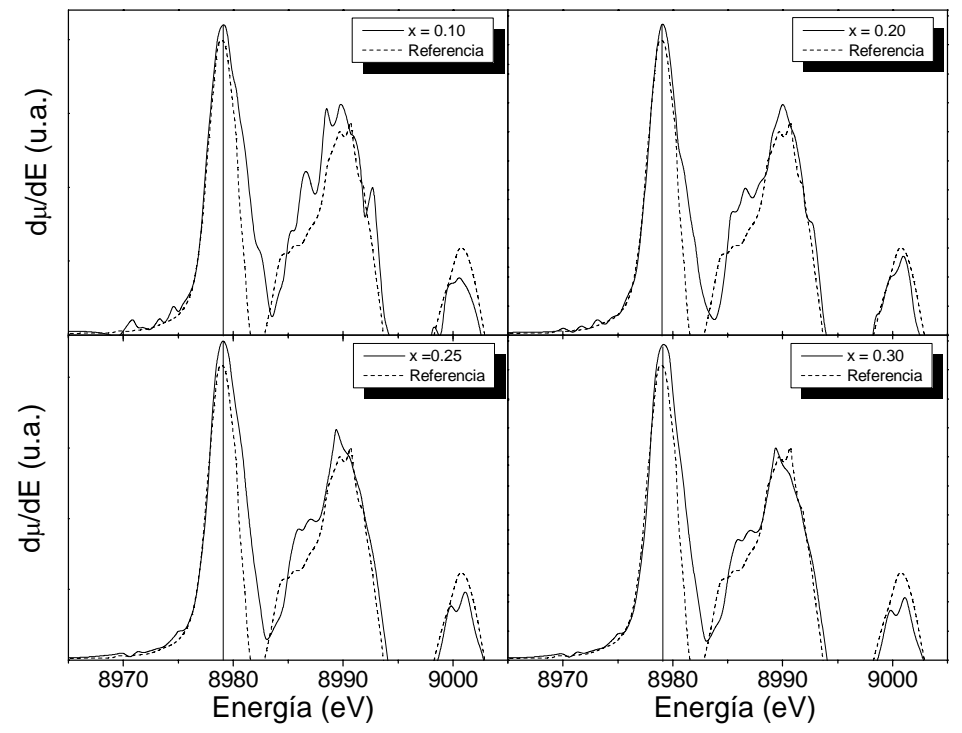

Figura 5.22: Comparación de la derivada de los espectros de absorción en la región XANES (línea sólida) con las correspondientes al $\mathrm{Cu}$ metálico (línea a trozos). La línea vertical indica la energía del borde $\mathrm{K}$ del $\mathrm{Cu}$ $\left(\mathrm{E}_{\mathrm{b}}=8979 \mathrm{eV}\right)^{22}$

Como se puede observar de las figuras 5.21 y 5.22, que la energía del borde de las muestras coinciden con la energía para los bordes de absorción del $\mathrm{Fe}$ y del $\mathrm{Cu}$ metálicos ${ }^{22}$ respectivamente, indicando que ninguno de estos dos metales se encuentra oxidado.

A continuación en la figura 5.23 se muestran los espectros XAS normalizados correspondientes al borde $K$ del Cu para las muestras con $\mathrm{x}=0.10, \mathrm{x}=0.20, \mathrm{x}=0.25 \mathrm{y} \mathrm{x}=0.30$.

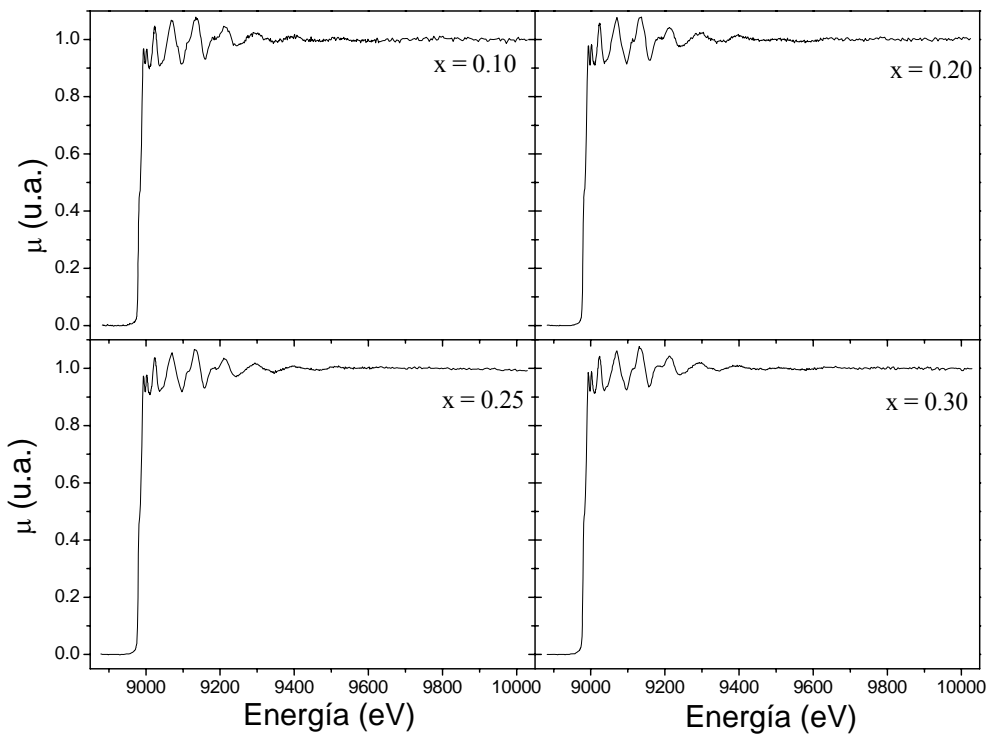

Figura 5.23: Espectros de absorción de rayos X para distintas concentraciones. 
Para el estudio de la región extendida del espectro de absorción se procedió con el tratamiento de los espectros mostrados en la figura 5.23 como se detalla en el Capítulo 4. La figura 5.24 muestra la transformada de Fourier (TF) de los mismos. Se nota que, a medida que la concentración de $\mathrm{Cu}$ aumenta, la intensidad de la señal correspondiente a la primera esfera de coordinación $(1.7 \leq \mathrm{R} \leq 2.6 \AA$ A ), decrece; mientras que la distancia a primeros vecinos se corre hacia valores más altos indicando una expansión de la red.

Las distancias interatómicas (D) se incrementan linealmente con el porcentaje de $\mathrm{Cu}$ presente en las muestras, como puede ser observado de la Tabla 5.6 y en la figura 5.25 donde se presentan los resultados de los ajustes.

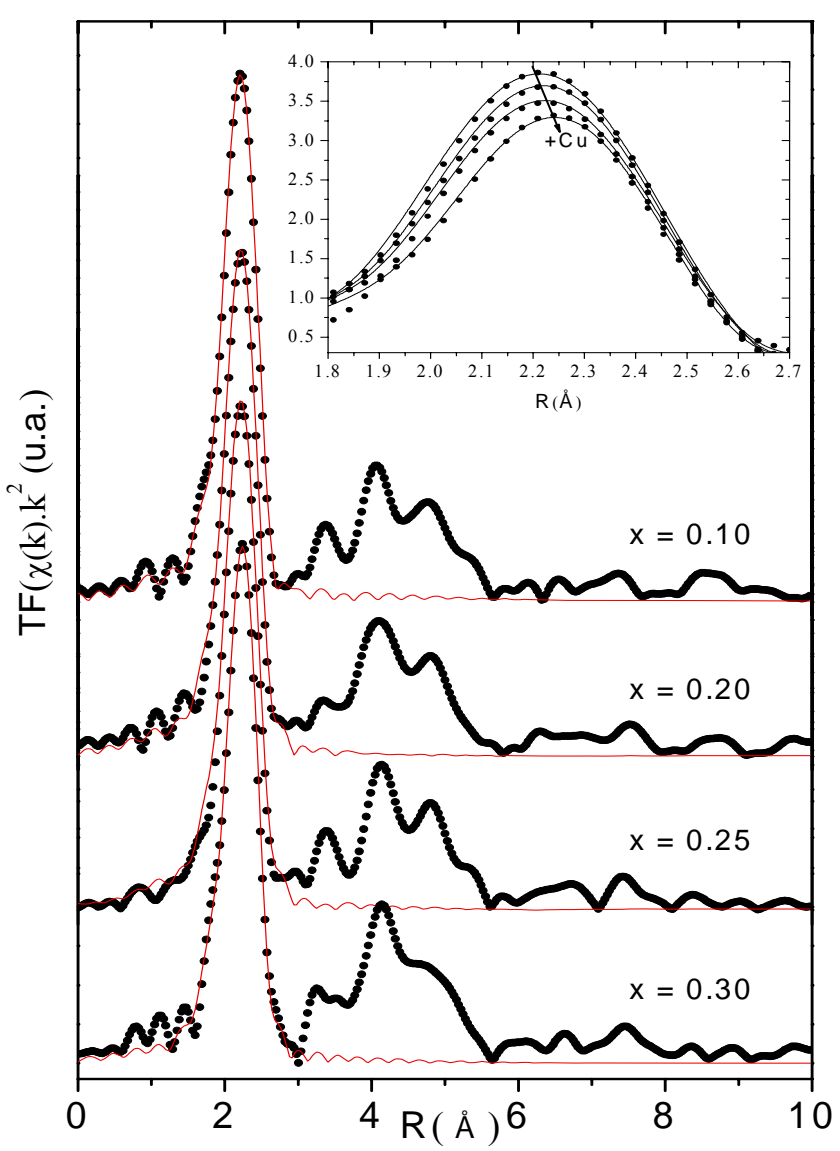

Figura 5.24: Transformada de Fourier de la región EXAFS de los espectros medidos en el borde $K$ del $\mathrm{Cu}$ junto con el ajuste (línea roja). El recuadro es una ampliación del pico correspondiente a la primera esfera de coordinación. 


\begin{tabular}{|c|c|c|c|}
\hline $\mathrm{x}$ & $\mathrm{D}(\AA)$ & $\mathrm{N}_{\mathrm{Cu}}$ & $\mathrm{N}^{*} \mathrm{Cu}$ \\
\hline 0.10 & $2.545_{2}$ & $1.5_{6}$ & 1.30 \\
\hline 0.20 & $2.551_{2}$ & $2.8_{7}$ & 2.62 \\
\hline 0.25 & $2.554_{2}$ & $3.5_{7}$ & 3.26 \\
\hline 0.30 & $2.556_{2}$ & $4.2_{6}$ & 3.96 \\
\hline
\end{tabular}

Tabla 5.6: Distancia a primeros vecinos (D), número de átomos de $\mathrm{Cu}$ primeros vecinos, determinado por EXAFS $\left(\mathrm{N}_{\mathrm{Cu}}\right)$ y número medio de átomos de $\mathrm{Cu}$ primeros vecinos estadístico $\left(\mathrm{N}_{\mathrm{Cu}}^{*}\right)$ en función del contenido de $\mathrm{Cu}$.

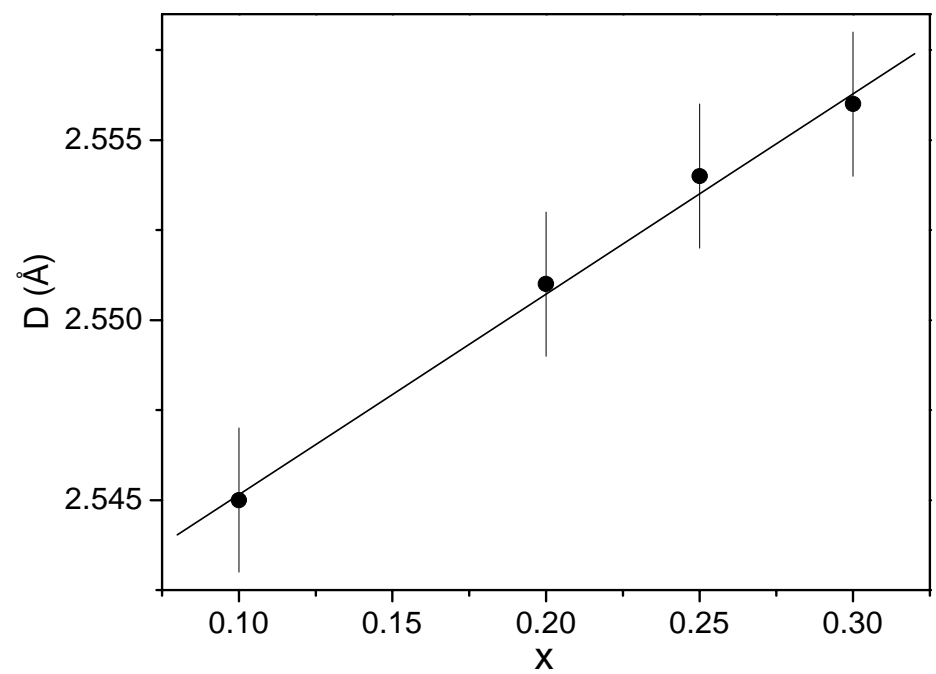

Figura 5.25: Variación de la distancia a primeros vecinos para las distintas concentraciones estudiadas obtenidas de los ajustes de los espectros EXAFS, junto con un ajuste lineal de los datos (línea sólida)

La distancia a primeros vecinos muestra un crecimiento lineal con el contenido de $\mathrm{Cu}$. Los resultados de un ajuste lineal indican que la distancia a primeros vecinos con el contenido de $\mathrm{Cu}$ crece según $5.6_{3} \times 10^{-4} \AA \AA \%$ at. de $\mathrm{Cu}$.

El número de átomos de $\mathrm{Cu}\left(N_{C u}\right)$ en la primera esfera de coordinación también muestra un cambio con el contenido de $\mathrm{Cu}$ (Tabla 5.6) como es esperable de la incorporación substitucional a la estructura $f c c$. La figura 5.26 muestra la variación del número de átomos de $\mathrm{Cu}$ en la primera esfera de coordinación en función del contenido de $\mathrm{Cu}$. Se observa un aumento lineal de $N_{C u}$ con el aumento de $\mathrm{x}$, confirmando que los átomos de $\mathrm{Cu}$ se incorporan substitucionalmente en la red fcc. 


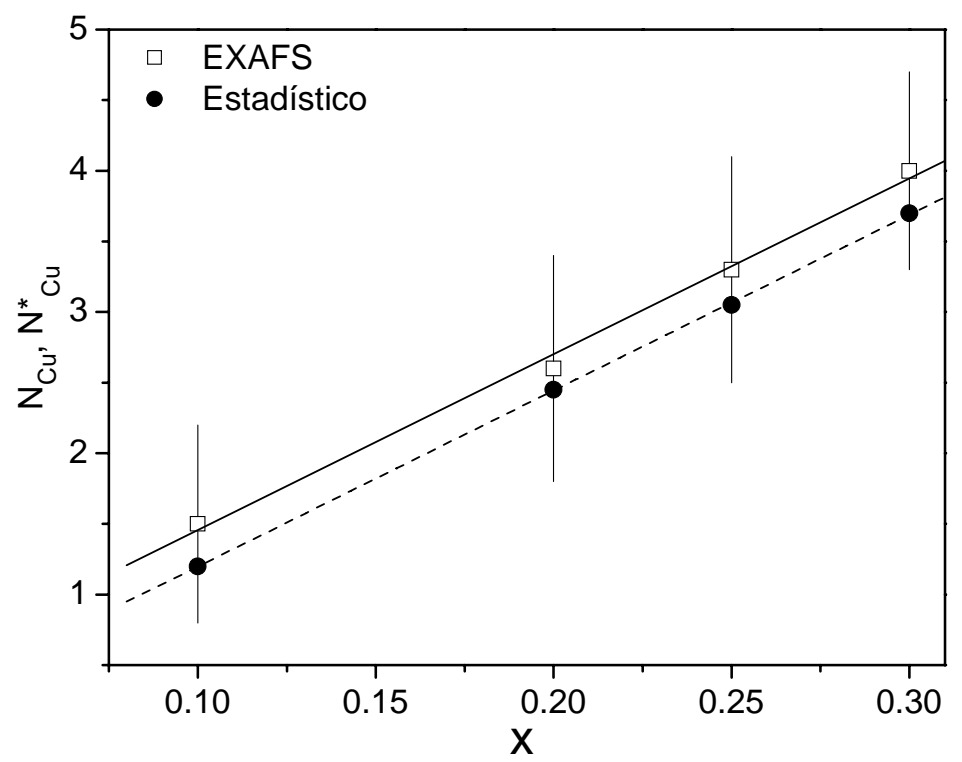

Figura 5.26: Valores de $N_{\mathrm{Cu}}$ obtenidos de los ajustes EXAFS (cuadrados abiertos) y según la predicción estadística $\left(N^{*}{ }_{\mathrm{Cu}}\right)$ (círculos sólidos), junto con los ajustes lineales correspondientes (línea sólida) y (línea punteada), respectivamente.

\section{Modelo estadístico de distribución atómica}

A continuación se procedió a calcular el número más probable de átomos de $\mathrm{Cu}$ primeros vecinos en una estructura $f c c$ suponiendo que los átomos están distribuidos aleatoriamente en los sitios cristalográficos de la estructura. La probabilidad de encontrar un átomo que posea un número $n_{\mathrm{Fe}}, n_{\mathrm{Mn}}$ y $n_{\mathrm{Cu}}$ de primeros vecinos $\mathrm{Fe}, \mathrm{Mn}$ y $\mathrm{Cu}$, respectivamente, viene dada por la siguiente expresión:

$$
P\left(n_{F e}, n_{M n}, n_{C u}\right)=\frac{N !}{n_{F e} ! n_{M n} ! n_{C u} !}\left(p_{F e}\right)^{n_{F e}}\left(p_{M n}\right)^{n_{M n}}\left(p_{C u}\right)^{n_{C u}}
$$

donde $N=12$ es el número total de primeros vecinos, en la red $f c c$, y $p_{F e}, p_{M n}$ y $p_{C u}$ los porcentajes atómicos de cada elemento en la muestra. Para determinar cuál es la probabilidad de tener $n_{C u}$ átomos de $\mathrm{Cu}$ primeros vecinos para una concentración dada, se fijó este valor en la expresión anterior y se realizó una sumatoria sobre todas las posibles combinaciones entre los valores $n_{F e} \mathrm{y}$ $n_{M n}$ que cumplan con la condición $n_{F e}+n_{M n}+n_{C u}=12$. En la figura 5.27, se muestran los resultados obtenidos para la probabilidad de encontrar $n_{C u}$ átomos de $\mathrm{Cu}$ primeros vecinos para las distintas 
concentraciones estudiadas, junto con un ajuste de los resultados empleando una distribución de tipo log-normal.
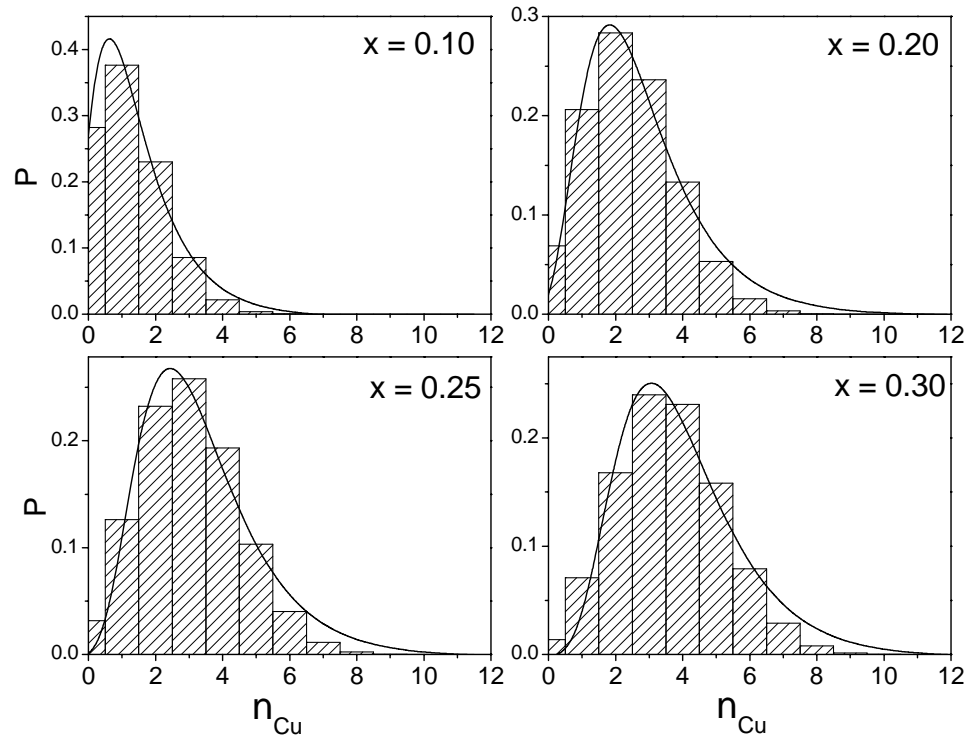

Figura 5.27: Probabilidad de encontrar $n_{\mathrm{Cu}}$ átomos de Cu primeros vecinos según lo predicho por la Ec.5.1 para las distintas concentraciones estudiadas. La línea continua representa el ajuste log-normal de las probabilidades calculadas.

El máximo determinado en cada uno de los ajustes $\left(N^{*}{ }_{\max }\right)$ es el valor más probable de $n_{\mathrm{Cu}}$ para una determinada concentración de $\mathrm{Cu}$. A partir de éste se puede estimar el valor medio para el número de primeros vecinos usando la siguiente relación:

$$
\left.N_{\mathrm{Cu}}^{*}=\exp \left[\ln \left(N_{\max }^{*}\right)\right]+\sigma^{2} / 2\right]
$$

donde $\sigma$ es la dispersión de la distribución log-normal. Dado que el número de átomos de $\mathrm{Cu}$ primeros vecinos obtenidos por EXAFS $\left(N_{\mathrm{Cu}}\right)$ es un valor promedio, $N_{\mathrm{Cu}}^{*}$ es el valor más apropiado para hacer una comparación entre los mismos. Los resultados obtenidos para $N_{\mathrm{Cu}}^{*}$ son mostrados en la Tabla 5.6. En la figura 5.26 se representaron los valores calculados $N_{\mathrm{Cu}}^{*}$ empleando la distribución aleatoria de átomos en la red $f c c$ junto con los determinados experimentalmente por EXAFS $\left(N_{\mathrm{Cu}}\right)$. Como se observa en los ajustes lineales propuestos para $N_{\mathrm{Cu}}$ y para $N_{\mathrm{Cu}}^{*}$, la variación de éstos con $\mathrm{x}$ es idéntica con una tasa de crecimiento de $0.12_{2} n_{\mathrm{Cu}} / \%$ at. de $\mathrm{Cu}$. 


\subsubsection{Estudio hiperfino}

\section{Espectroscopía Mössbauer a temperatura ambiente}

En la figura 5.28 se muestran los espectros Mössbauer registrados a temperatura ambiente (TA) en el rango de velocidades $[-8,8 \mathrm{~mm} / \mathrm{s}]$ para todas las composiciones. En los espectros con $\mathrm{x}$ $=0.00, \mathrm{x}=0.10 \mathrm{y} \mathrm{x}=0.15$ se puede observar una pequeña fracción de un sextete de alto campo hiperfino. Éste es asociado a la formación de la fase $b c c-\mathrm{Fe}(\mathrm{Mn}, \mathrm{Cu})$, la cual también fue observada en los difractogramas. Esta interacción magnética fue reproducida con una distribución de campos magnéticos hiperfinos (DCMH) con un valor medio de campo $\left(B_{h f}\right)$ de $31 \mathrm{~T}$ y un corrimiento isomérico $(\delta) \mathrm{de}-0.01 \mathrm{~mm} / \mathrm{s}$ (ver Tabla 5.7), ambos menores a los correspondientes al bcc-Fe puro $\left(B_{h f}=33 \mathrm{~T}\right.$ y $\left.\delta=0.00 \mathrm{~mm} / \mathrm{s}\right) .{ }^{9}$ De acuerdo a la Ref. [23], reemplazar un átomo de Fe por uno de $\mathrm{Mn}$ en la estructura $b c c$ del Fe modifica el campo hiperfino en aproximadamente $-2 \mathrm{~T}$ por cada átomo de $\mathrm{Mn}$ presente en la primera esfera de coordinación del $\mathrm{Fe}$, mientras que el corrimiento isomérico se ve afectado en $-0.01 \mathrm{~mm} / \mathrm{s}$ en el sistema FeMn. Un efecto similar fue observado en el campo hiperfino del bcc-Fe con la incorporación de Cu. ${ }^{24} \mathrm{En}$ muestras de $\left(\mathrm{Fe}_{1-\mathrm{x}} \mathrm{Mn}_{\mathrm{x}}\right)_{90} \mathrm{Cu}_{10}$ con $(\mathrm{x}=$ $0.05 \mathrm{y} \mathrm{x}=0.10)$ se encontró que el efecto de tener un átomo de $\mathrm{Cu}$ primer vecino al átomo absorbente de Fe disminuye el campo hiperfino de la fase $b c c$ en aproximadamente $1 \mathrm{~T}$ y causa un aumento de $0.01 \mathrm{~mm} / \mathrm{s}$ en el corrimiento isomérico. ${ }^{25}$ Por lo tanto el valor de $31 \mathrm{~T}$ obtenido puede ser atribuido a estructuras bcc del tipo $\mathrm{Fe}(\mathrm{Mn})$ y/o $\mathrm{Fe}(\mathrm{Mn}, \mathrm{Cu})$. Según Ref. [26] un campo de $31 \mathrm{~T}$ puede obtenerse cuando la fracción de $\mathrm{Mn}$, en relación al $\mathrm{Fe}$, sea de sólo un 10 \%at., aproximadamente; esto sugiere que, si bien la mezcla de los elementos alcanzó su estado estacionario a las $15 \mathrm{~h}$ de molienda, existe una variación composicional entre distintas partes de la muestra. Dado que para las concentraciones $\mathrm{x}=0.10 \mathrm{y} \mathrm{x}=0.15$ un modelo de distribución estadística indica que el número de átomos de $\mathrm{Cu}$ primeros vecinos en la fase bcc es aproximadamente igual a 1, sumado a que el efecto del $\mathrm{Cu}$ sobre los parámetros hiperfinos es menor al producido por los átomos de Mn y al bajo porcentaje de la contribución de alto campo al espectro, hace difícil poder afirmar si el Cu está o no formando parte de la estructura bcc. 

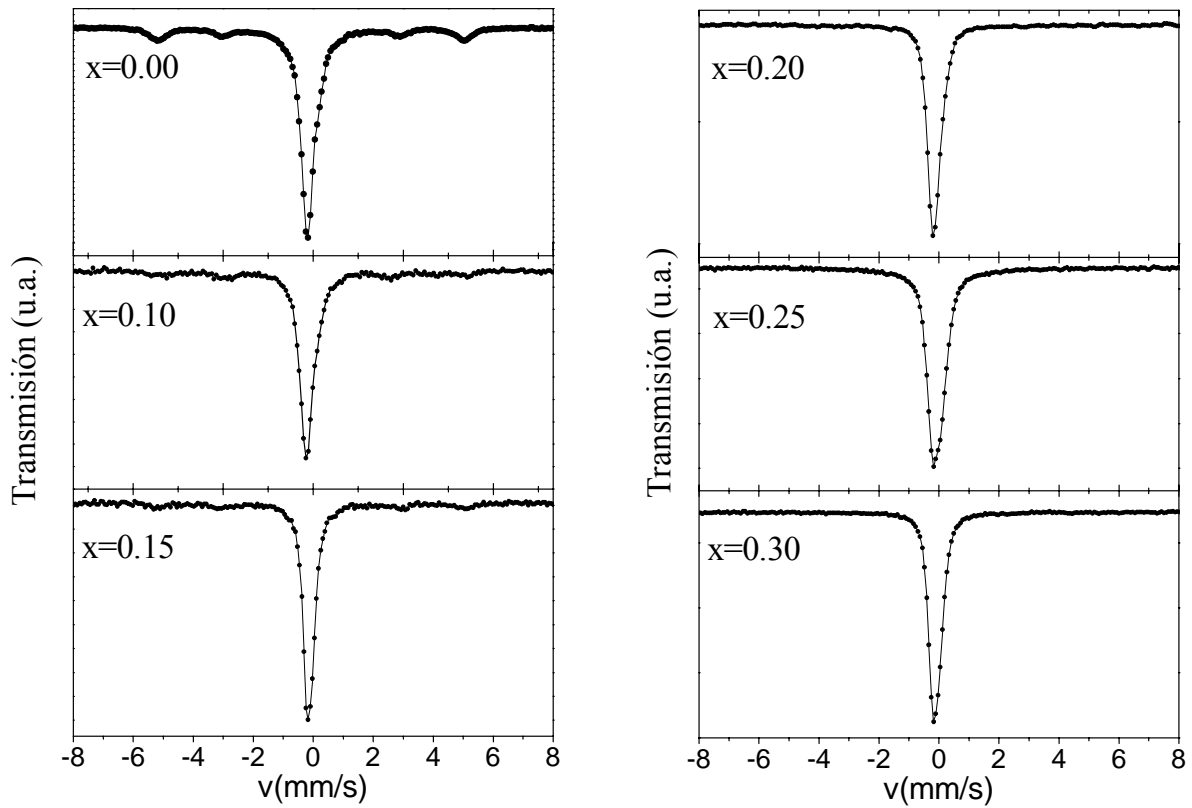

Figura 5.28: Espectros Mössbauer en el rango mayor de velocidades. Las líneas sólidas corresponden al resultado de los ajustes.

\begin{tabular}{|c|c|c|c|c|}
\cline { 2 - 5 } \multicolumn{1}{c|}{} & \multicolumn{4}{c|}{ Interacción de alto campo } \\
\hline $\mathrm{x}$ & $\mathrm{B}_{\mathrm{hf}}(\mathrm{T})$ & $\sigma(\mathrm{T})$ & $\delta(\mathrm{mm} / \mathrm{s})$ & $\mathrm{f}$ \\
\hline 0.00 & 31.1 & 7.2 & -0.01 & 0.18 \\
\hline 0.10 & 31.0 & 7.2 & -0.01 & 0.10 \\
\hline 0.15 & 31.1 & 7.3 & -0.01 & 0.05 \\
\hline 0.20 & - & - & - & - \\
\hline 0.25 & - & - & - & - \\
\hline 0.30 & - & - & - & - \\
\hline
\end{tabular}

Tabla 5.7: Resultados del ajuste para el campo magnético hiperfino medio $\left(\mathrm{B}_{\mathrm{hf}}\right)$, momento de $2^{\mathrm{do}}$ orden $(\sigma)$, corrimiento isomérico medio $(\delta)$ y fracción relativa $(\mathrm{f})$ al total del espectro para la interacción de alto campo.

A continuación se muestran los espectros Mössbauer obtenidos en el rango de velocidades [$2,2 \mathrm{~mm} / \mathrm{s}$ ]. Como se puede observar en la figura 5.29, los espectros muestran una estructura hiperfina compleja, la cual puede ser asociada a la diversidad de entornos del átomo de $\mathrm{Fe}$ presentes. Todos los espectros poseen una línea central ancha y ligeramente asimétrica, la cual se corre hacia valores positivos de velocidad a medida que el contenido de $\mathrm{Cu}$ aumenta. Ya que se ha tenido especial cuidado en la preparación de los absorbentes para evitar problemas originados por un absorbente grueso ${ }^{27}$ se pueden descartar efectos en el ancho de línea debido a ésto. En el caso de las muestras $\mathrm{x}=0.00, \mathrm{x}=0.10 \mathrm{y} \mathrm{x}=0.15$ la línea central está ubicada entre las líneas internas de la contribución de alto campo mencionada anteriormente, interacción que fue contemplada al momento de realizar los ajustes en este rango de velocidades. 


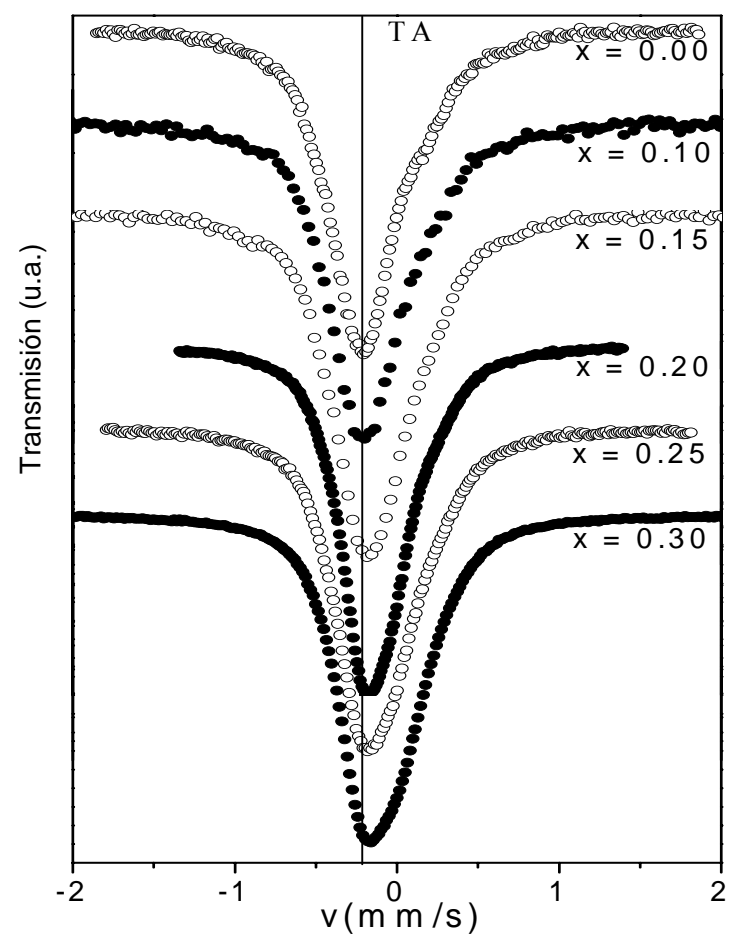

Figura 5.29: Espectros Mössbauer medidos en el rango de velocidades menor. La línea vertical indica el valor del corrimiento isomérico para la muestra $\mathrm{x}=0.00$.

Los espectros correspondientes a $\mathrm{Fe}_{79} \mathrm{Mn}_{21}$ han sido reproducidos por otros autores empleando una distribución de campos magnéticos hiperfinos de valor medio próximo a $2 \mathrm{~T}$ para muestras preparadas por horno de $\operatorname{arco}^{28} \mathrm{o}$ con sextetes no resueltos para muestras preparadas por métodos convencionales, ${ }^{29}$ siendo en todos los casos el corrimiento isomérico igual a $-0.09 \mathrm{~mm} / \mathrm{s}$. En el caso de muestras preparadas por aleado mecánico, los espectros fueron reproducidos con un sextete no resuelto $\left(\mathrm{B}_{\mathrm{hf}}=2.1 \mathrm{~T}\right)$ asociado a la fase antiferromagnética $f_{c c}$-FeMn y una línea simple asociada con la presencia de fallas de apilamiento de la estructura $f c c$, ambas interacciones con el mismo corrimiento isomérico $(\delta=-0.08 \mathrm{~mm} / \mathrm{s}){ }^{30,31}$ Como fuera comentado en el Capítulo 4 , luego de proponer varios modelos de ajuste, para reproducir la parte central de los espectros se optó por una distribución de campos magnéticos hiperfinos entre 0 T y 30 T dividido en 40 subespectros con un ancho de línea de $0.25 \mathrm{~mm} / \mathrm{s}$ cada uno, permitiendo que la probabilidad a campo cero pudiese ser no nula para contemplar así contribuciones paramagnéticas producidas por la presencia de pequeños granos con comportamiento superparamagnético, efectos de superficie y/o defectos estructurales en la fase $f c c$. Es válido mencionar que se intentó agregar una línea simple con el mismo corrimiento isomérico que la distribución (fijando en cero la probabilidad de distribución a campo nulo), asociada a la presencia de átomos de Fe en fallas de apilamiento en la estructura $f c c$, no observándose una mejoría notable en el resultado final del ajuste en comparación con el modelo sin esta contribución paramagnética. En consecuencia, el modelo más simple fue adoptado para la 
reproducción de todo el conjunto de espectros, es decir, una DCMH con una probabilidad no nula a campo cero y correlación entre el campo hiperfino y el corrimiento isomérico. Los resultados del ajuste de los espectros a temperatura ambiente junto con las correspondientes distribuciones de probabilidades son presentados en la figura 5.30. La Tabla 5.8 muestra los valores obtenidos para $B_{h f}$ y $\delta$ para las distintas concentraciones de $\mathrm{Cu}$.
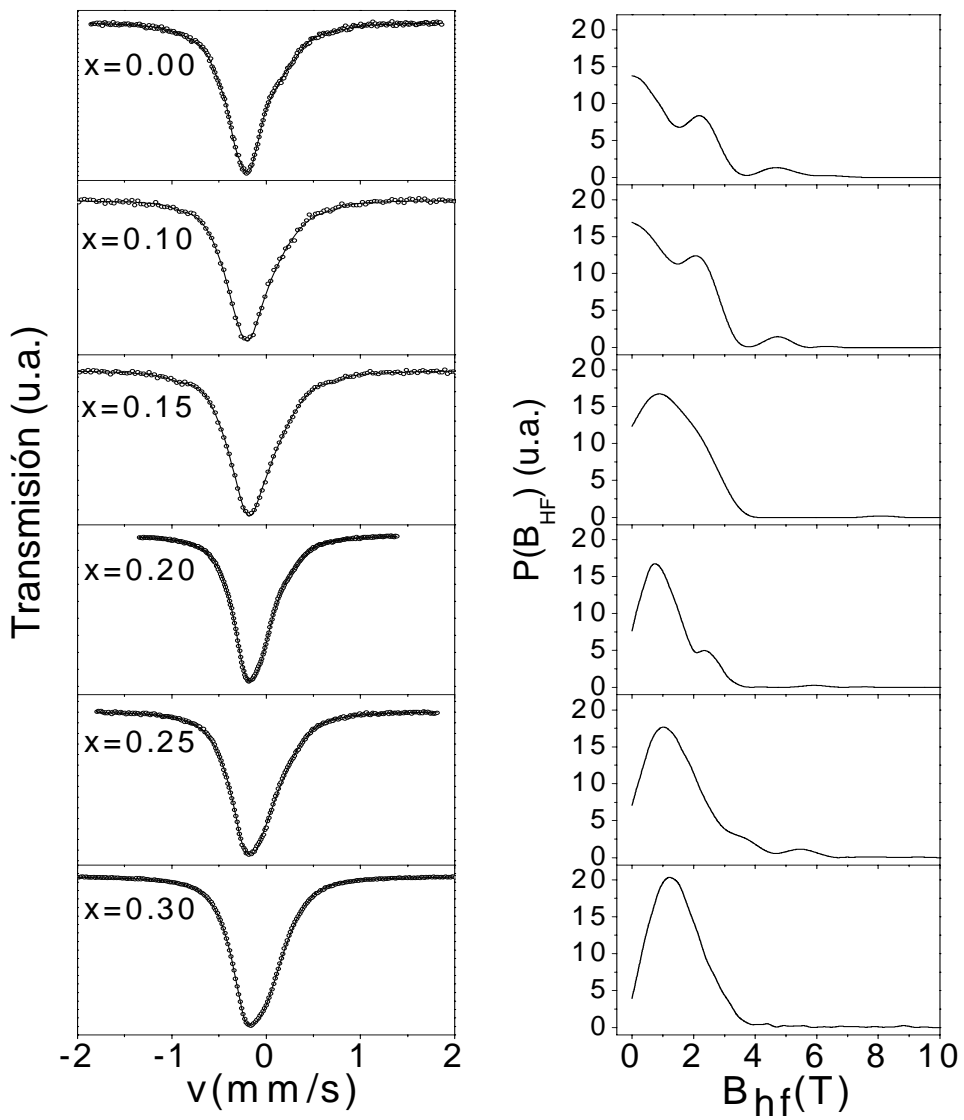

Figura 5.30: Espectros Mössbauer medidos en el rango menor de velocidades junto con las correspondientes DCMH. La línea continua en los espectros representa el ajuste.

\begin{tabular}{|c|c|c|c|c|}
\hline $\mathrm{x}$ & $\mathrm{B}_{\mathrm{hf}}(\mathrm{T})$ & $\sigma(\mathrm{T})$ & $\delta(\mathrm{mm} / \mathrm{s})$ & $\mathrm{f}$ \\
\hline 0.00 & 1.5 & 1.2 & -0.05 & 0.81 \\
\hline 0.10 & 1.5 & 1.1 & -0.03 & 0.90 \\
\hline 0.15 & 1.4 & 1.0 & -0.01 & 0.95 \\
\hline 0.20 & 1.4 & 0.9 & 0.00 & 1.00 \\
\hline 0.25 & 1.3 & 0.9 & 0.01 & 1.00 \\
\hline 0.30 & 1.3 & 0.8 & 0.03 & 1.00 \\
\hline
\end{tabular}

Tabla 5.8: Resultados de los ajustes. Campo hiperfino medio $\left(\mathrm{B}_{\mathrm{hf}}\right)$, momento de $2^{\mathrm{do}}$ orden $(\sigma)$, corrimiento isomérico medio $(\delta)$ y fracción relativa al total del espectro (f) para la interacción de bajo campo. 
Se puede apreciar en la figura 5.30 que las distribuciones devienen más estrechas y pierden su estructura cuando el contenido de $\mathrm{Cu}$ aumenta. La DCMH refleja la presencia de numerosos entornos para los átomos de $\mathrm{Fe}$ en la fase $f c c$. Como se observó previamente en el estudio de la cinética de molienda, el valor de la probabilidad a campo cero muestra un comportamiento interesante. Ésta posee valores muy altos para la muestra $\sin \mathrm{Cu}$ y paulatinamente disminuye a medida que el contenido de $\mathrm{Cu}$ aumenta. En base a este comportamiento particular, es posible descartar que esta contribución pueda ser asociada a partículas en estado superparamagnético dado que, al poseer todas las muestras tamaños de cristalita similares, la contribución a campo cero tendría que ser similar independientemente del contenido de $\mathrm{Cu}$. Por la misma razón, también se puede descartar que esta contribución pueda provenir del desorden en la superficie de las partículas. Otra hipótesis posible para explicar este comportamiento está basada en la presencia de fallas de apilamiento en la fase $f c c$. La energía necesaria para crear una falla de apilamiento se incrementa a razón de $1.18 \mathrm{~mJ} / \%$ peso de $\mathrm{Cu}^{32}$ Con esto en mente, es factible atribuir la contribución a campo nulo de la DCMH a la presencia de fallas de apilamiento creadas por el método de preparación. Las fallas de apilamiento en una estructura $f c c$ forman una estructura local del tipo hcp. Debido a que este tipo de fallas se presentan por lo general en forma aislada, es muy difícil encontrar un arreglo con la periodicidad lo suficientemente importante como para poder ser detectadas por DRX. Sin embargo, la presencia de las fallas de apilamiento puede ser detectada indirectamente observando cómo se desplazan las líneas de difracción. ${ }^{33}$ Dicho corrimiento posee la particularidad de afectar en forma distinta a las distintas líneas de la estructura, tal como se muestra en las siguientes expresiones, que predicen el desplazamiento de las líneas (111) y (200) pertenecientes a la estructura $f c c$ en función de la fracción de fallas de apilamiento $(\alpha)$ :

$$
\begin{aligned}
& \Delta(2 \theta)_{(111)}^{\circ}=\frac{90 \sqrt{3 \alpha} \tan \left(\theta_{111}\right)}{4 \pi^{2}} \\
& \Delta(2 \theta)_{(200)}^{\circ}=-\frac{90 \sqrt{3 \alpha} \tan \left(\theta_{200}\right)}{2 \pi^{2}}
\end{aligned}
$$

De las expresiones anteriores se puede notar que, en presencia de una determinada fracción de fallas de apilamiento, la línea (111) se desplaza hacia ángulos mayores; mientras que la línea (200) se desplaza el doble en sentido opuesto. Teniendo en cuenta ésto, se estudió el comportamiento de las líneas (111) y (200) en los difractogramas para las muestras con distintas cantidades de $\mathrm{Cu}$ y se observó que dichas líneas no estaban en la posición angular esperada. Si bien parte de este efecto puede ser atribuido al aumento del parámetro de red a medida que se incrementa el contenido de $\mathrm{Cu}$, el corrimiento en sentido opuesto, proveniente de las fallas de 
apilamiento permite que se puedan separar ambas contribuciones. Para ver el efecto de las fallas de apilamiento sobre las líneas se determinó el parámetro de red basándose en la posición angular de las reflexiones (111) y (200). Estos resultados se muestran en la figura 5.31.

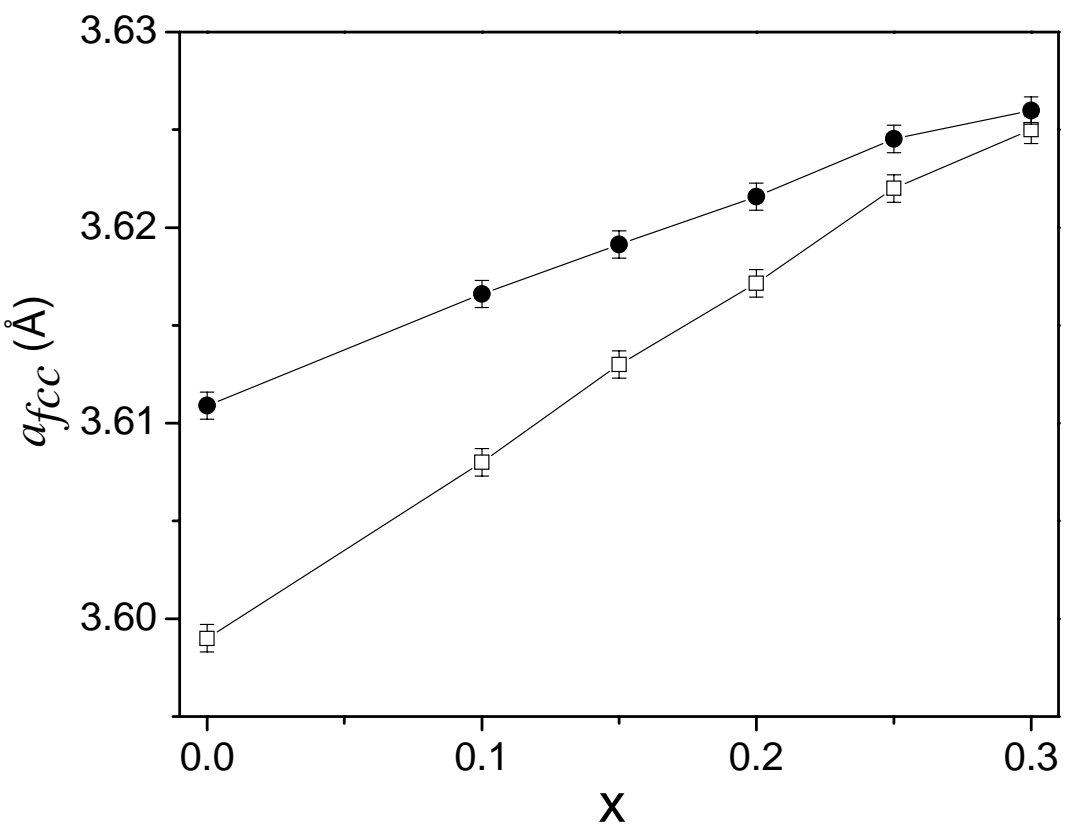

Figura 5.31: Parámetro de red determinado a partir de las posiciones angulares des las líneas (111) (cuadrados abiertos) y (200) (círculos sólidos).

En la figura anterior se observa que el parámetro de red obtenido, empleando la posición de la línea principal (111) es inferior al determinado cuando se emplean las posiciones correspondientes a la línea (200). Si el corrimiento de las líneas se debe a una variación en el parámetro de red con el contenido de $\mathrm{Cu}$, los parámetros de red determinados con ambas líneas deberían coincidir. Por lo tanto, esta diferencia es un claro indicio de que el efecto observado no es producto de un cambio en la constante de red. A su vez, que el parámetro de red correspondiente a la línea (200) sea mayor que el de (111) indica que, la línea (200) se encuentra a ángulos menores que los esperados o la línea (111) se encuentra a ángulos mayores a los esperados o ambas circunstancias se están presentando. Todo esto está en acuerdo con lo predicho por los corrimientos angulares esperados por la presencia de fallas de apilamiento. Por último, es de destacar que la diferencia entre los valores del parámetro de red obtenidos a partir de ambas líneas disminuye con el contenido de $\mathrm{Cu}$, siendo ésto indicativo de una disminución de la cantidad de fallas de apilamiento, lo cual está de acuerdo con el incremento de la energía necesaria para producir una falla a medida que se tiene más $\mathrm{Cu}$ en las muestras. Por lo tanto, la contribución a $B_{h f}=0$ puede atribuirse a las fallas de apilamiento. 
La figura 5.32 muestra el comportamiento de los parámetros $B_{h f}$ y $\delta$. Se observa que el valor $B_{h f}$ disminuye levemente mientras que un comportamiento opuesto es presentado por $\delta$, aumentando a medida que se incrementa el contenido de $\mathrm{Cu}$. Los valores encontrados, tanto para el $B_{h f}$ como para $\delta$ en la muestra $\sin \mathrm{Cu}$ son muy cercanos a los reportados por otros autores en muestras preparadas también por molienda mecánica. ${ }^{30,31} \mathrm{El}$ decrecimiento de $B_{h f}$ con el contenido de $\mathrm{Cu}$ puede explicarse basándose en la substitución en la red cristalina de un átomo magnético (Fe o $\mathrm{Mn}$ ) por uno no magnético $(\mathrm{Cu})$, lo cual produce una disminución del campo magnético efectivo que actúa sobre el núcleo absorbente. Haciendo uso de la relación obtenida en la sección del modelado estadístico se obtiene que el valor de $B_{h f}$ por cada átomo de $\mathrm{Cu}$ en la primera esfera de coordinación del $\mathrm{Fe}$ es de $-0.053_{2} \mathrm{~T}$. Los valores obtenidos del corrimiento isomérico permiten descartar la posibilidad de la incorporación del $\mathrm{Fe}$ en la estructura $f c c$ del $\mathrm{Cu}^{34}$ o la formación de una solución sólida $\mathrm{Mn}(\mathrm{Fe}) .{ }^{35} \mathrm{La}$ evolución de $\delta$ hacia valores positivos es una clara evidencia de la presencia del $\mathrm{Cu}$ en la aleación, siendo este parámetro hiperfino más sensible que el campo hiperfino medio. En este sentido, el aumento de $\mathrm{Cu}$ en las muestras da lugar a un aumento en la población de los electrones de la banda de conducción y, por consiguiente, a un aumento en la densidad de electrones $3 d$ alrededor del núcleo de Fe. Si la densidad electrónica de la capa $3 d$ de Fe aumenta, el apantallamiento de los electrones $s$ en el núcleo también aumentará; luego la densidad de electrones $s$ dentro del volumen nuclear disminuirá y en consecuencia se ésto se verá reflejado como un aumento en el corrimiento isomérico. Otro posible aporte al comportamiento mostrado podría estar originado el aumento del parámetro de red detectado tanto por DRX como por EXAFS, este último caso observado como un aumento de la distancia entre primeros vecinos con el agregado de $\mathrm{Cu}$. Para determinar la viabilidad de esta hipótesis se calculó el coeficiente de expansión volumétrica a temperatura constante dado por: ${ }^{36}$

$$
C_{e v}=\left.\frac{\partial \delta}{\partial \ln V}\right|_{T}
$$

El coeficiente de expansión obtenido es $\mathrm{C}_{\mathrm{ev}}=0.05_{1} \mathrm{~mm} / \mathrm{s}$, que es un orden de magnitud inferior que los reportados, ${ }^{36}$ mostrando así que el comportamiento de $\delta$ no puede ser asociado, por lo menos en forma completa, a la expansión del parámetro de red.

Tomando en cuenta la variación del corrimiento isomérico observado en la figura 5.32 se puede establecer la siguiente relación lineal entre $\delta$ and x:

$$
\delta(\mathrm{x})=0.25_{2} \mathrm{x}-0.05_{1}(\mathrm{~mm} / \mathrm{s})
$$


$\mathrm{y}$, usando la relación entre la fracción de $\mathrm{Cu}$ y el número de átomos de $\mathrm{Cu}$ primeros vecinos $\left(\mathrm{N}^{*}{ }_{\mathrm{Cu}}\right)$, se determina que la sustitución de un átomo de $\mathrm{Fe}$ o $\mathrm{Mn}$ por uno de $\mathrm{Cu}$ produce un incremento de $0.020_{2} \mathrm{~mm} / \mathrm{s}$ en el corrimiento isomérico. Estos valores son comparables con los reportados previamente para la fase $f c c$ del sistema $\mathrm{FeCu}$ preparado por molienda mecánica. ${ }^{37}$
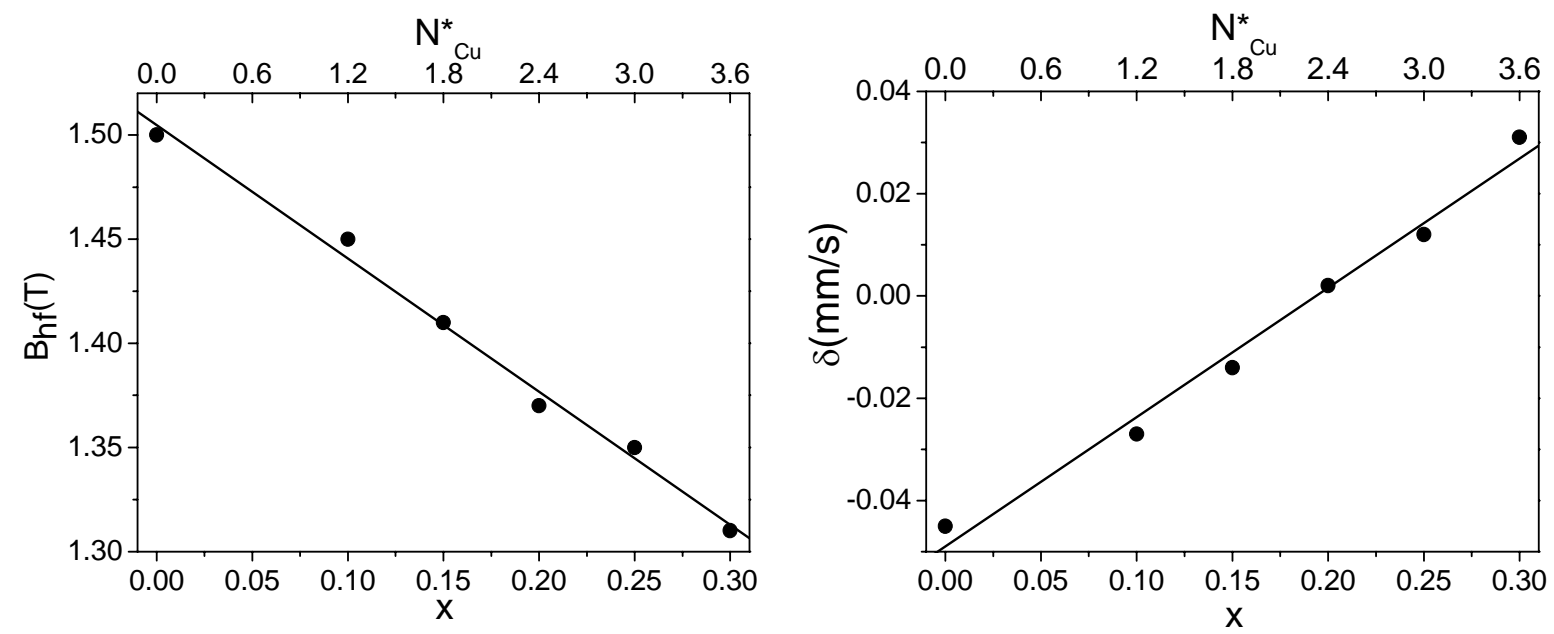

Figura 5.32: Parámetros obtenidos de los ajustes en función del contenido de $\mathrm{Cu}$. Campo hiperfino medio (izq.) y corrimiento isomérico medio (der.). La línea continua representa un ajuste lineal.

\section{Espectroscopía Mössbauer a bajas temperaturas}

Las medidas a bajas temperaturas fueron realizadas en el rango de velocidades entre [-4 , 4 $\mathrm{mm} / \mathrm{s}$ ] para las muestras con $\mathrm{x}=0.00, \mathrm{x}=0.10, \mathrm{x}=0.15 \mathrm{y} \mathrm{x}=0.20$, mientras que el rango tuvo que ser extendido a $[-5,5 \mathrm{~mm} / \mathrm{s}]$ para las muestras con $\mathrm{x}=0.25 \mathrm{y} \mathrm{x}=0.30$ debido a la gran relajación magnética que presentan los espectros. Los resultados del ajuste de los espectros para las distintas temperaturas, junto con las correspondientes distribuciones de probabilidades de campo hiperfino son presentados en las figuras 5.33 a 5.38 . 

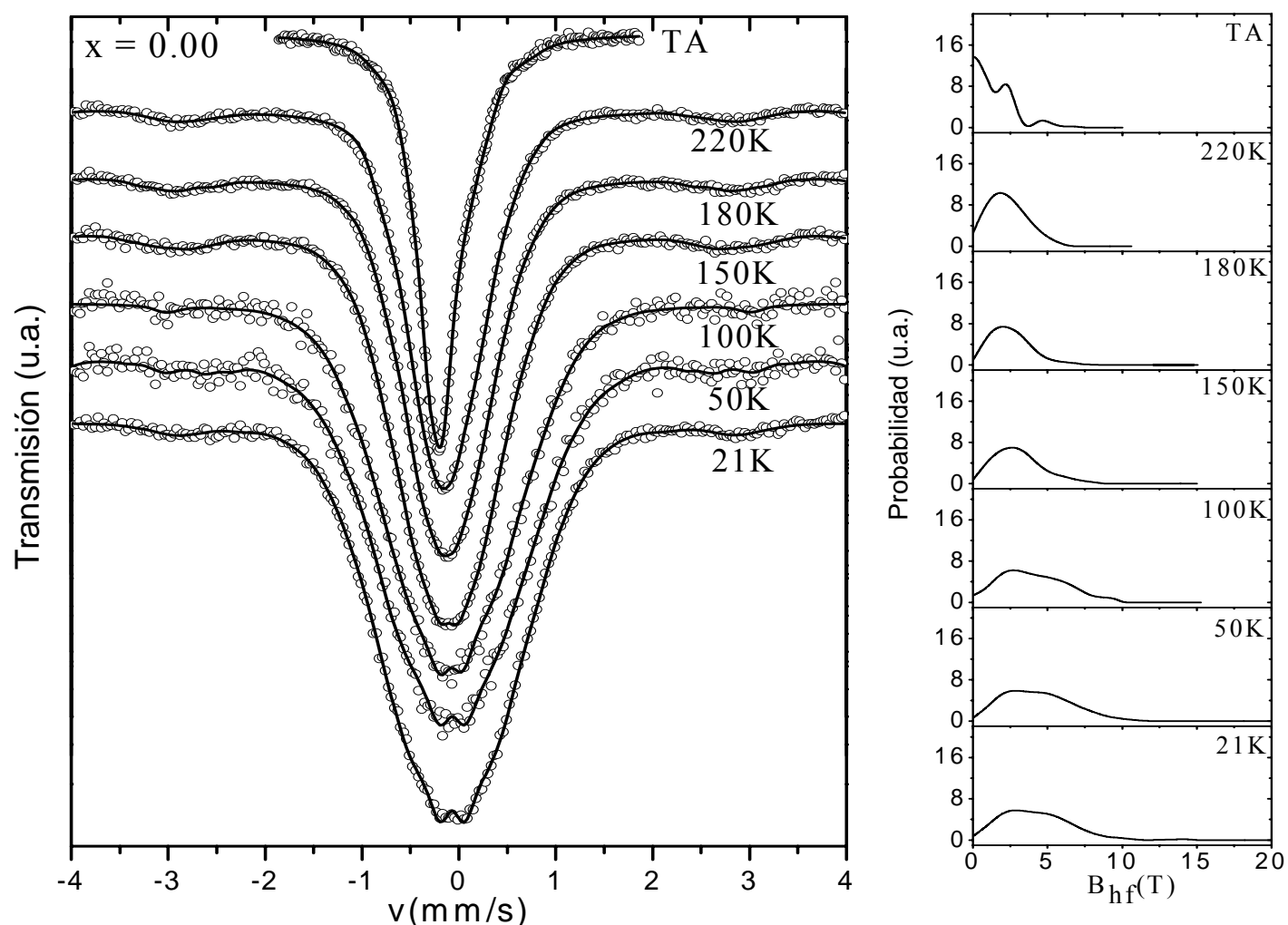

Figura 5.33: Espectros Mössbauer medidos a varias temperaturas para la muestra con $\mathrm{x}=0.00$ junto con las correspondientes DCMH. La línea continua en los espectros representa el ajuste.
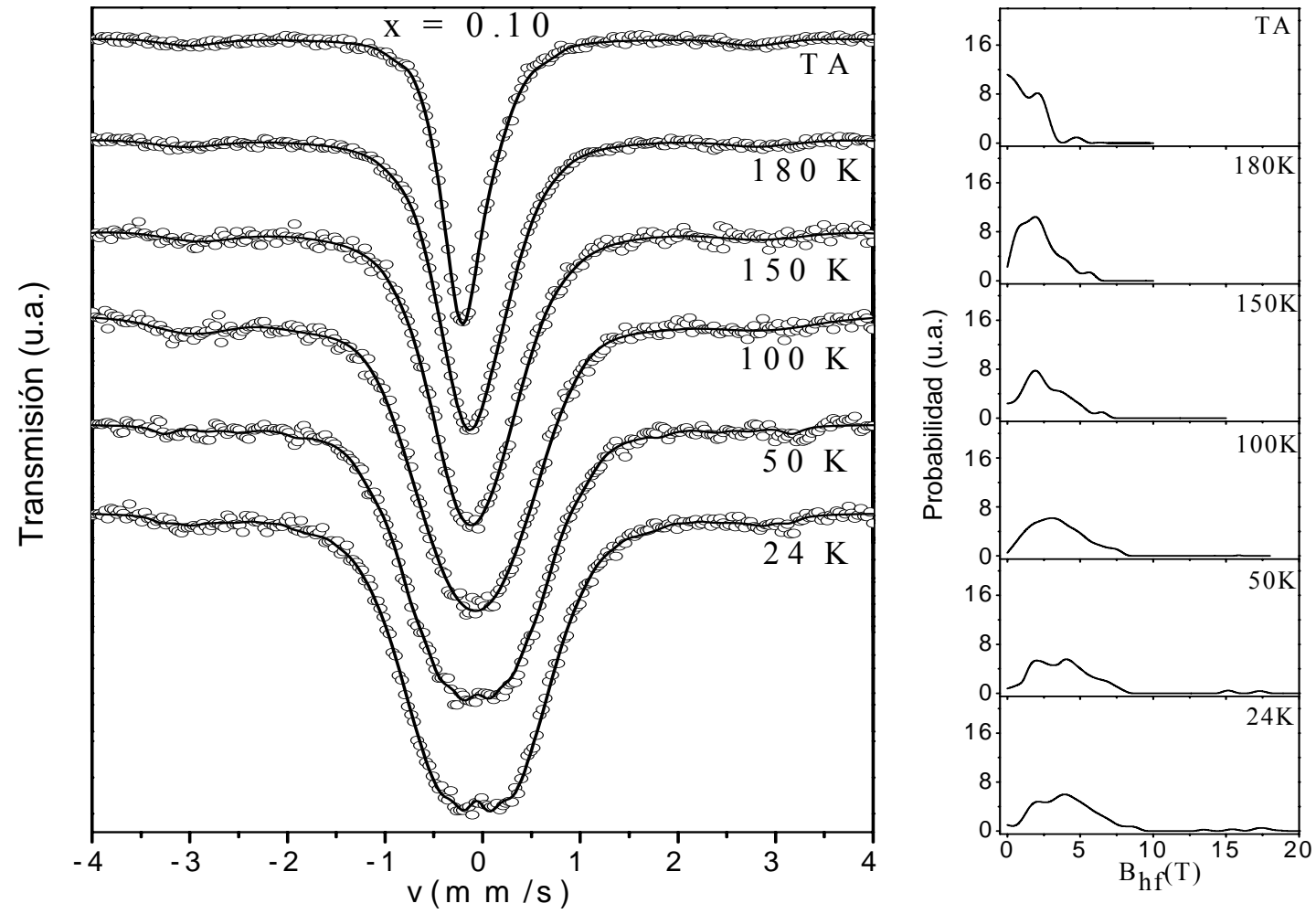

Figura 5.34: Espectros Mössbauer medidos a varias temperaturas para la muestra con $\mathrm{x}=0.10$ junto con las correspondientes DCMH. La línea continua en los espectros representa el ajuste. 

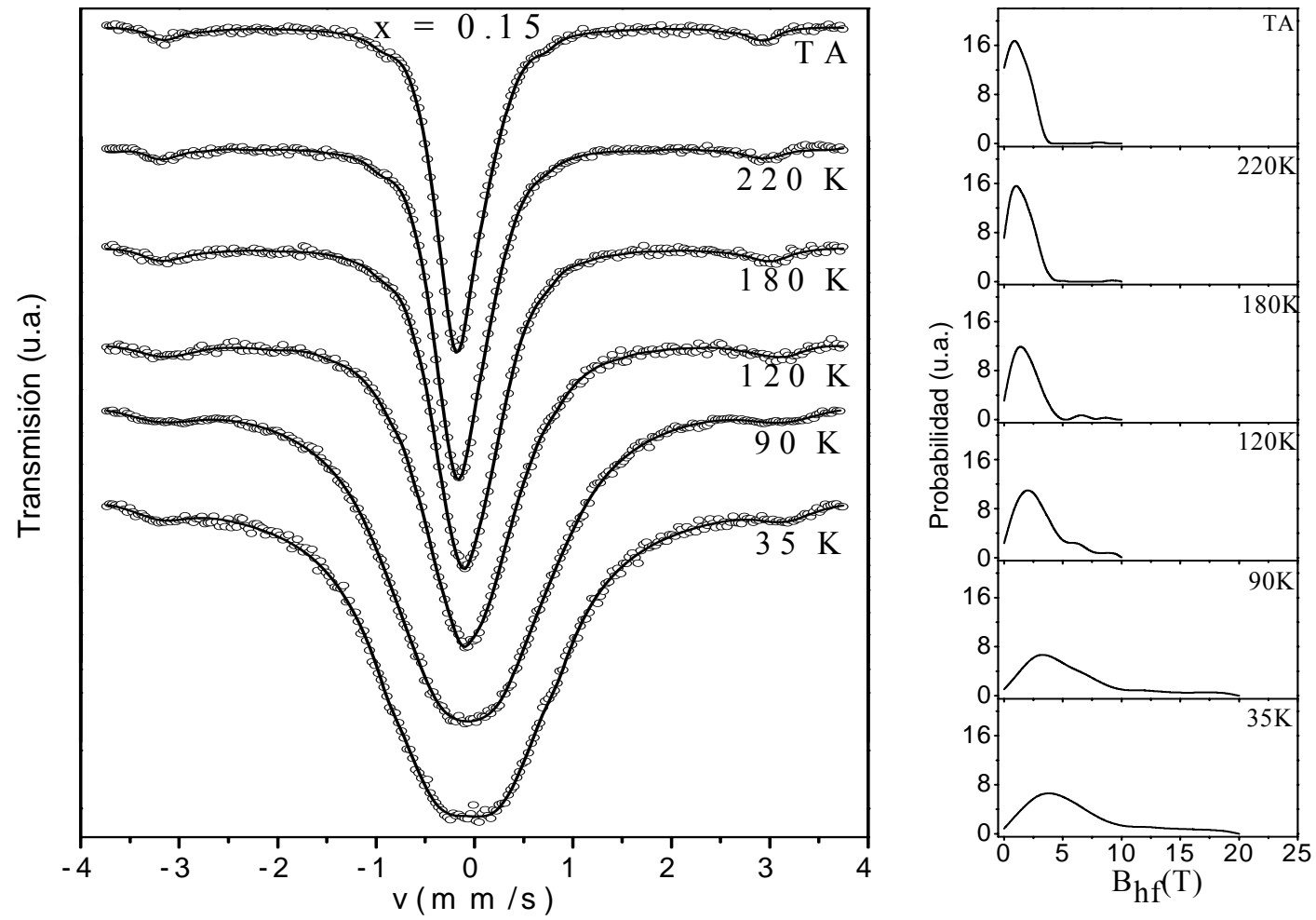

Figura 5.35: Espectros Mössbauer medidos a varias temperaturas para la muestra con $\mathrm{x}=0.15$ junto con las correspondientes DCMH. La línea continua en los espectros representa el ajuste.
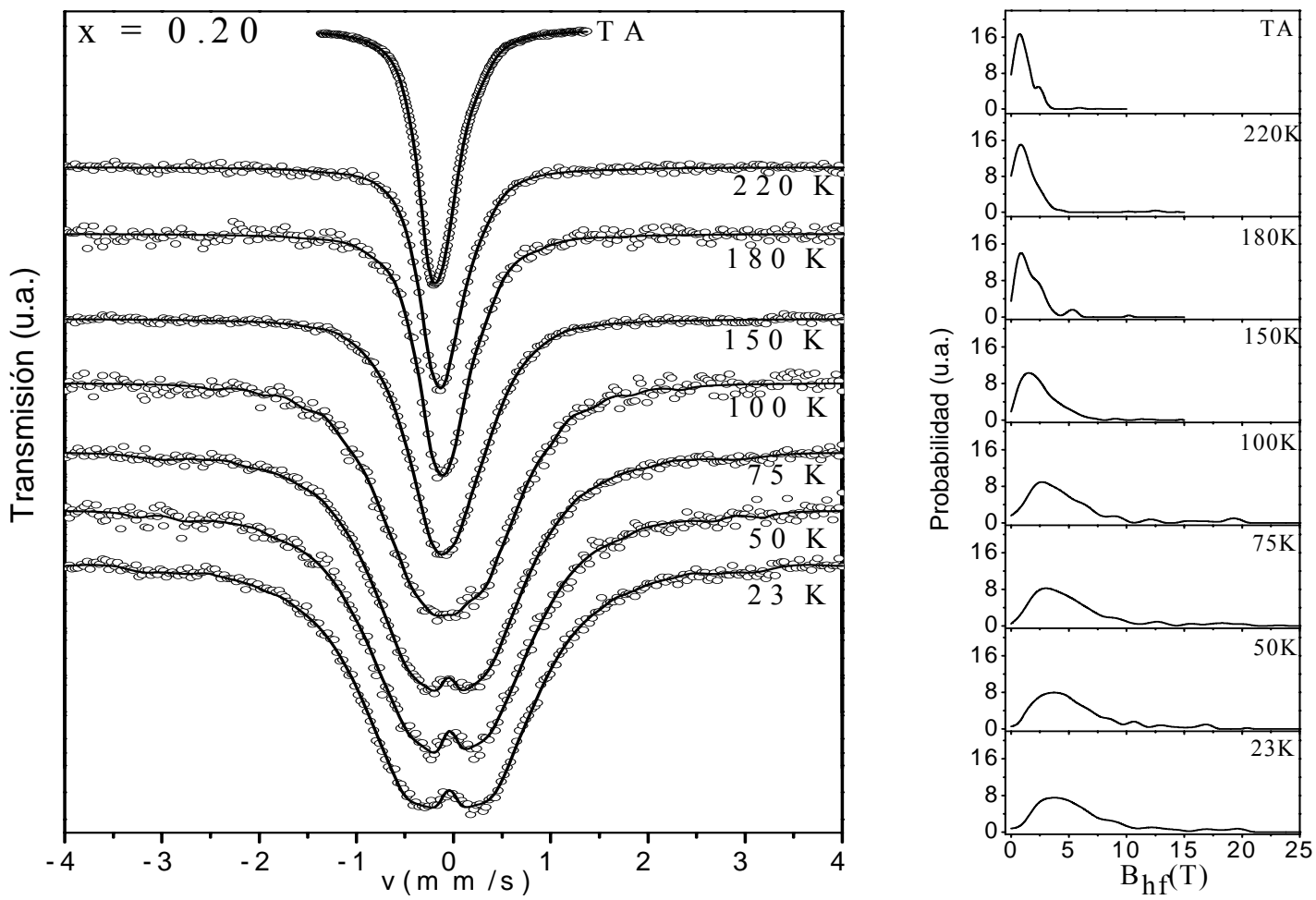

Figura 5.36: Espectros Mössbauer medidos a varias temperaturas para la muestra con $\mathrm{x}=0.20$ junto con las correspondientes DCMH. La línea continua en los espectros representa el ajuste. 

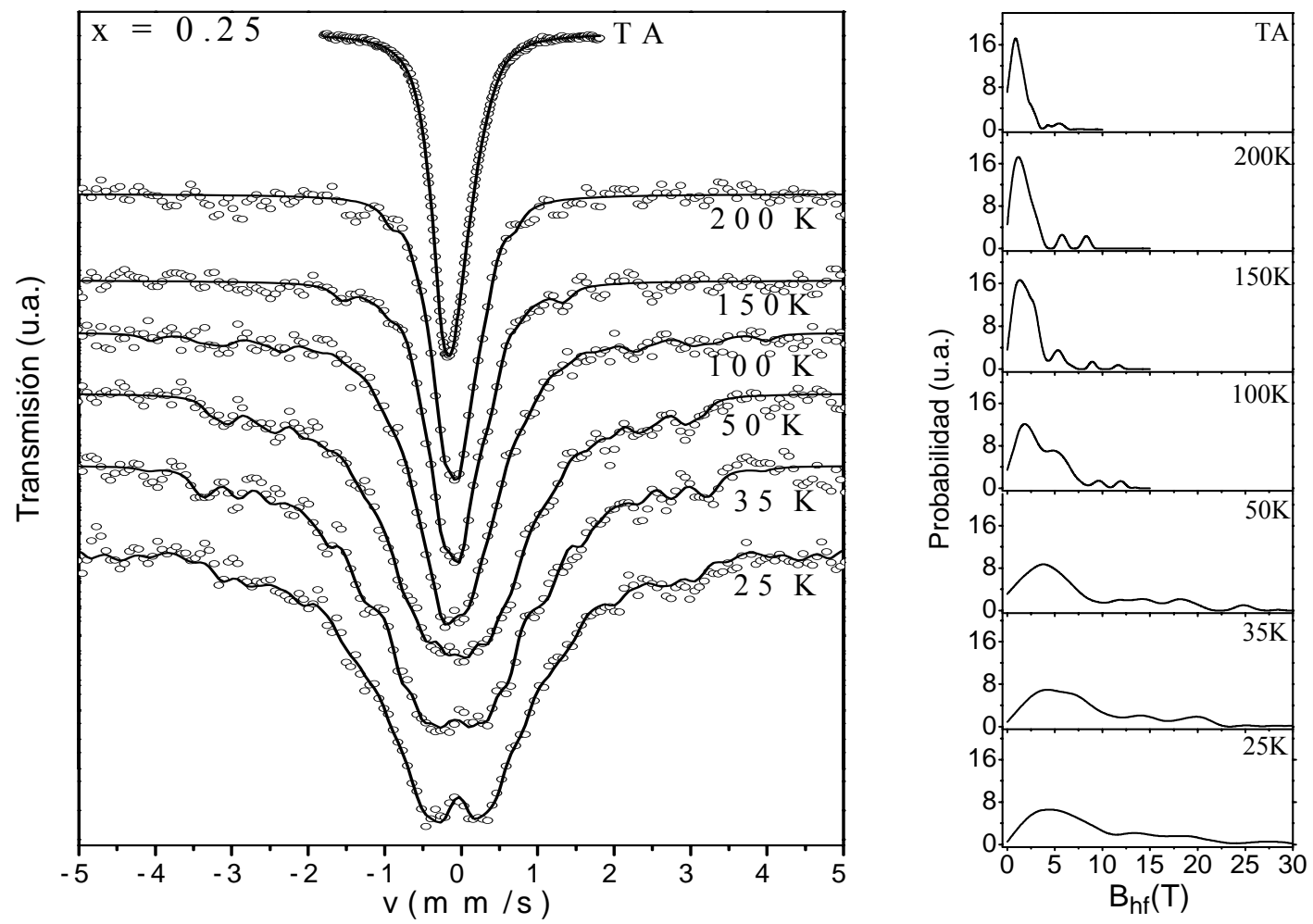

Figura 5.37: Espectros Mössbauer medidos a varias temperaturas para la muestra con $\mathrm{x}=0.25$ junto con las correspondientes DCMH. La línea continua en los espectros representa el ajuste.
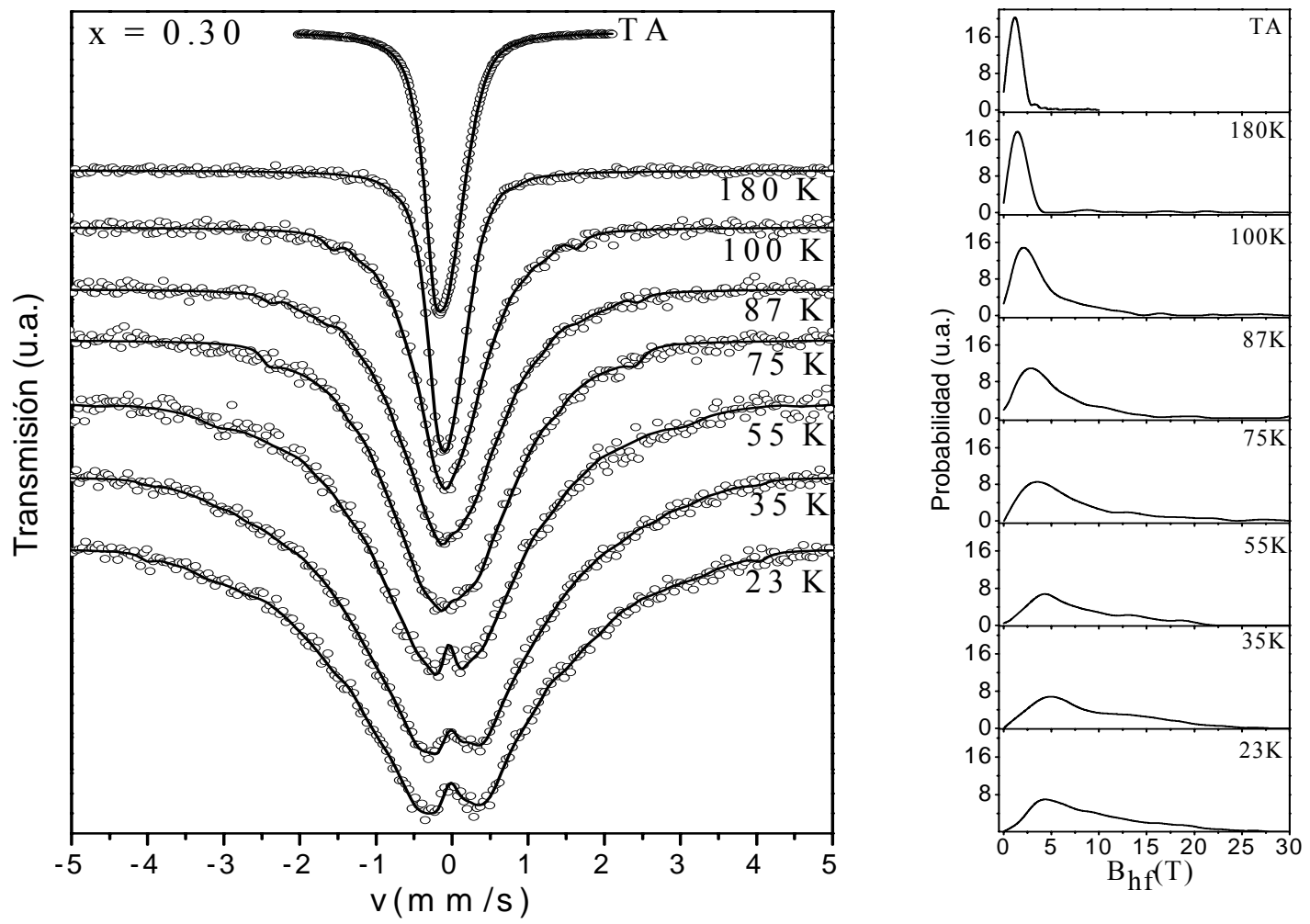

Figura 5.38: Espectros Mössbauer medidos a varias temperaturas para la muestra con $\mathrm{x}=0.30$ junto con las correspondientes DCMH. La línea continua en los espectros representa el ajuste. 
En Tabla 5.9 se presentan los parámetros obtenidos de los ajustes:

\begin{tabular}{|c|c|c|c|c|}
\hline $\mathrm{X}$ & $\mathrm{T}(\mathrm{K})$ & $\mathrm{B}_{\mathrm{hf}}(\mathrm{T})$ & $\sigma(\mathrm{T})$ & $\delta(\mathrm{mm} / \mathrm{s})$ \\
\hline \multirow{7}{*}{0.00} & TA & 1.5 & 1.2 & -0.05 \\
\hline & 220 & 2.0 & 1.3 & -0.02 \\
\hline & 180 & 2.5 & 1.4 & 0.00 \\
\hline & 150 & 3.0 & 1.6 & 0.01 \\
\hline & 100 & 3.5 & 2.1 & 0.03 \\
\hline & 50 & 3.8 & 2.2 & 0.03 \\
\hline & 21 & 4.0 & 2.3 & 0.04 \\
\hline \multirow{6}{*}{0.10} & TA & 1.5 & 1.1 & -0.03 \\
\hline & 180 & 2.1 & 1.3 & 0.02 \\
\hline & 150 & 2.7 & 1.5 & 0.03 \\
\hline & 100 & 3.6 & 1.9 & 0.04 \\
\hline & 50 & 4.5 & 2.7 & 0.04 \\
\hline & 23 & 4.8 & 3.0 & 0.05 \\
\hline \multirow{6}{*}{0.15} & TA & 1.4 & 1.0 & -0.01 \\
\hline & 220 & 1.5 & 1.1 & 0.02 \\
\hline & 180 & 2.1 & 1.4 & 0.03 \\
\hline & 120 & 4.1 & 2.0 & 0.04 \\
\hline & 90 & 4.7 & 2.5 & 0.05 \\
\hline & 35 & 5.4 & 3.2 & 0.05 \\
\hline \multirow{8}{*}{0.20} & TA & 1.4 & 0.9 & 0.00 \\
\hline & 220 & 1.4 & 1.6 & 0.03 \\
\hline & 180 & 1.5 & 1.7 & 0.04 \\
\hline & 150 & 2.6 & 1.9 & 0.05 \\
\hline & 100 & 4.4 & 2.9 & 0.05 \\
\hline & 75 & 5.1 & 3.6 & 0.06 \\
\hline & 50 & 5.7 & 3.8 & 0.06 \\
\hline & 23 & 6.2 & 4.2 & 0.06 \\
\hline \multirow{7}{*}{0.25} & TA & 1.3 & 0.9 & 0.01 \\
\hline & 200 & 1.6 & 1.7 & 0.04 \\
\hline & 150 & 2.0 & 2.1 & 0.05 \\
\hline & 100 & 5.7 & 2.6 & 0.06 \\
\hline & 50 & 8.0 & 4.3 & 0.07 \\
\hline & 35 & 8.4 & 4.8 & 0.07 \\
\hline & 25 & 8.6 & 4.9 & 0.07 \\
\hline \multirow{8}{*}{0.30} & TA & 1.3 & 0.8 & 0.03 \\
\hline & 180 & 1.4 & 1.6 & 0.07 \\
\hline & 100 & 4.8 & 4.1 & 0.08 \\
\hline & 87 & 5.9 & 4.3 & 0.09 \\
\hline & 75 & 6.9 & 4.8 & 0.09 \\
\hline & 55 & 8.1 & 5.1 & 0.09 \\
\hline & 35 & 9.2 & 5.5 & 0.09 \\
\hline & 23 & 9.6 & 5.6 & 0.09 \\
\hline
\end{tabular}

Tabla 5.9: Resultados de los ajustes para las distintas temperaturas y concentraciones. 
A pesar de la reducción de la temperatura, los espectros continúan sin resolverse aún a la más baja temperatura alcanzada $(21 \mathrm{~K})$, presentando la señal un importante ensanchamiento, el cual comienza a distintas temperaturas de acuerdo con el contenido de $\mathrm{Cu}$, llegando a tener contribuciones de hasta $30 \mathrm{~T}$ para $\mathrm{x}=0.30$ a la temperatura más baja. La presencia de una componente hiperfina magnética tan ancha y no resuelta sobre una región tan extensa de temperaturas, refleja la presencia de una gran cantidad de entornos diferentes para los átomos de $\mathrm{Fe}$, producto de tener números diferentes de átomos de $\mathrm{Cu}$ y $\mathrm{Mn}$ como primeros vecinos y la existencia del desorden topológico. La figura 5.39 muestra los resultados obtenidos para el campo hiperfino medio en función de la temperatura para las distintas concentraciones de $\mathrm{Cu}$. Como se puede apreciar, para todas las muestras, $B_{h f}$ se incrementa al disminuir la temperatura. Esta temperatura es dependiente de la concentración, presentándose a menores valores cuanto mayor es la concentración de $\mathrm{Cu}$. Este comportamiento sugiere que algún proceso de orden o bloqueo magnético está ocurriendo.

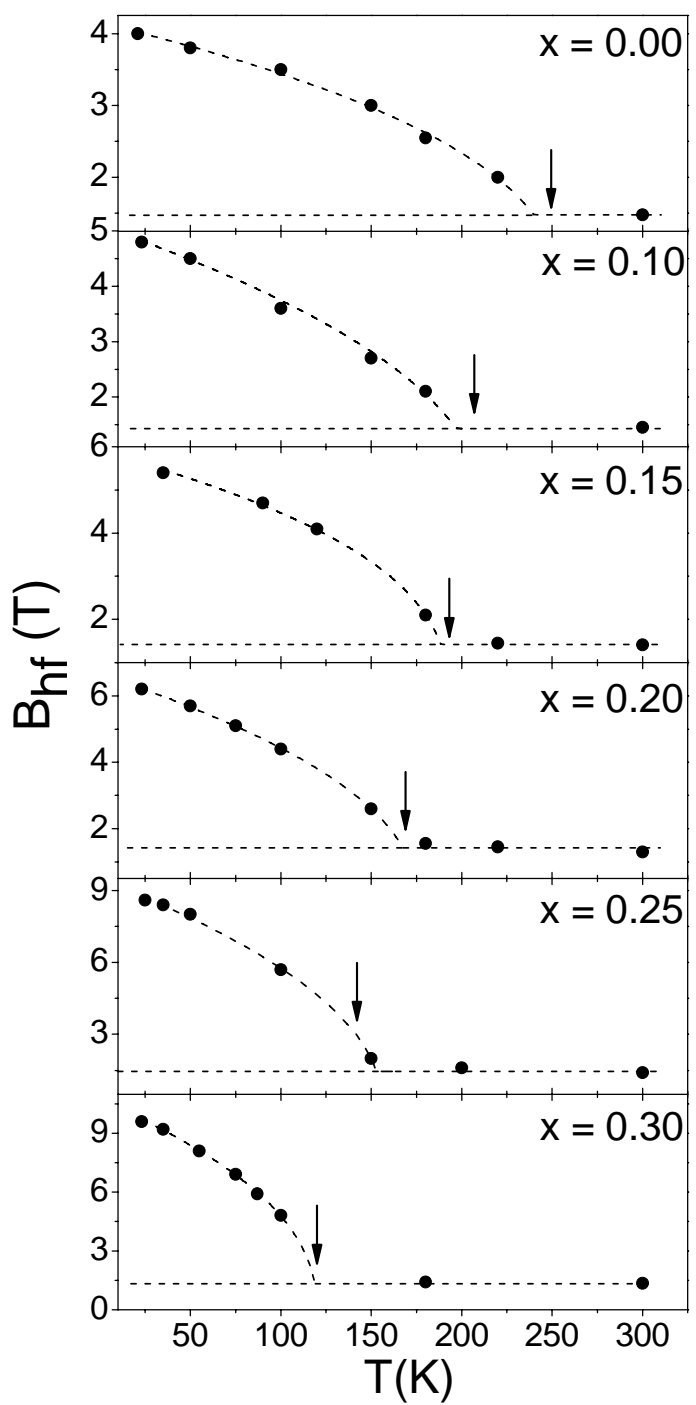

Figura 5.39: Evolución con la temperatura del campo hiperfino medio. Las líneas punteadas son una guía para la vista. 
A medida que se incrementa el contenido de $\mathrm{Cu}$ el corrimiento isomérico aumenta, tendencia que se mantiene en todo la región de temperaturas estudiadas (figura 5.40). Tal y como fue atribuido en las muestras medidas a temperatura ambiente, este comportamiento puede estar originado tanto por la diferencia de electrones aportados por el $\mathrm{Cu}$ con respecto al $\mathrm{Fe} \mathrm{y} \mathrm{al} \mathrm{Mn}$, como por la expansión del parámetro de red con el contenido de $\mathrm{Cu}$. El comportamiento de $\delta$ con la temperatura puede ser explicado en base a la contracción que experimenta la red cristalina con el descenso de temperatura y a los efectos contemplados en el corrimiento Doppler de segundo orden.

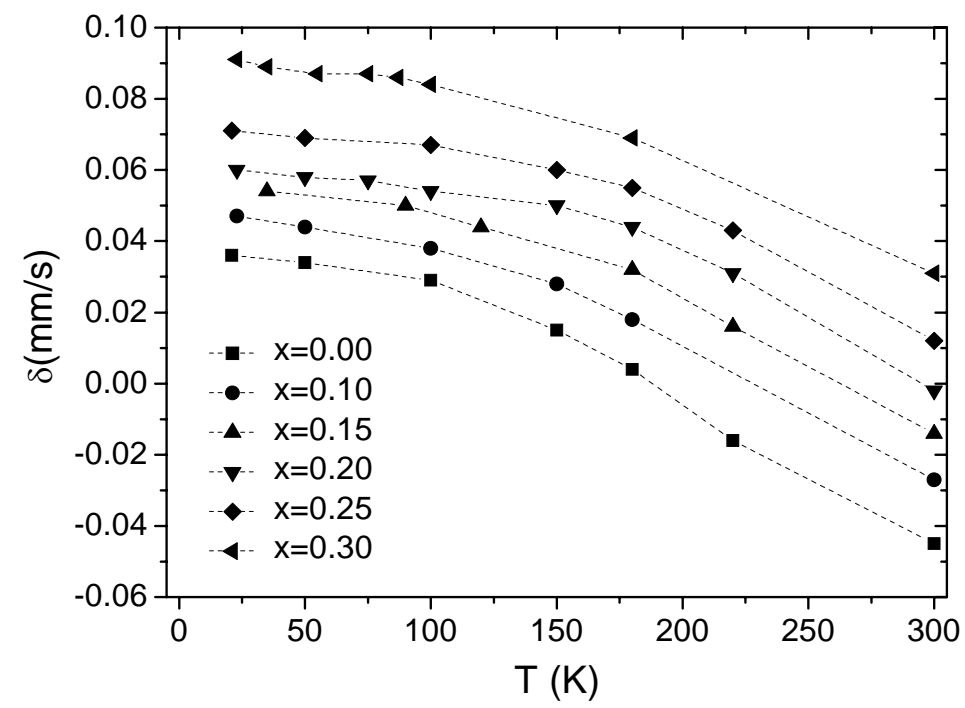

Figura 5.40: Evolución con la temperatura del corrimiento isomérico medio. Las líneas punteadas son una guía para la vista. 


\subsubsection{Estudio magnético}

En esta sección se presentan y discuten los resultados de las medidas magnéticas, las cuales comprenden la dependencia de la susceptibilidad magnética con la temperatura con la frecuencia y con el campo magnético aplicado, ciclos de histéresis y medidas de magnetización con y sin campo aplicado en función de la temperatura.

\section{Susceptibilidad magnética AC}

Las medidas de susceptibilidad magnética AC se realizaron en función de la temperatura entre $20 \mathrm{~K}$ y $320 \mathrm{~K}$. La dependencia con la temperatura de la componente en fase de la susceptibilidad $\left(\chi^{\prime}\right)$ se presenta en la figura 5.41. De acuerdo al contenido de $\mathrm{Cu}$ se pueden diferenciar dos comportamientos. El primero se observa para las muestras con $\mathrm{x}=0.00, \mathrm{x}=0.10 \mathrm{y}$ $\mathrm{x}=0.15$ donde $\chi^{\prime}$ se incrementa levemente cuando aumenta la temperatura, mostrando un cambio de pendiente a una temperatura $T_{\max }$ igual a $250 \mathrm{~K}, 205 \mathrm{~K}$ y $190 \mathrm{~K}$, respectivamente. Para temperaturas superiores a $T_{\max }$ las curvas no muestran cambios significativos, lo que está asociado a la presencia de una fase ferromagnética ordenada, ${ }^{38}$ estando esto de acuerdo con la presencia de la fase bcc por DRX y EM. En el segundo grupo compuesto por las muestras con $0.20 \leq \mathrm{x} \leq 0.30$, este cambio de comportamiento comienza a ser más pronunciado a medida que la concentración de $\mathrm{Cu}$ aumenta, evidenciándose la aparición de un máximo. También, como en el caso de las muestras del primer grupo, la posición de este máximo depende de la concentración, desplazándose hacia temperaturas menores a medida que el contenido de $\mathrm{Cu}$ crece. A continuación, la Tabla 5.10 muestra las temperaturas a las cuales aparecen estos máximos. 


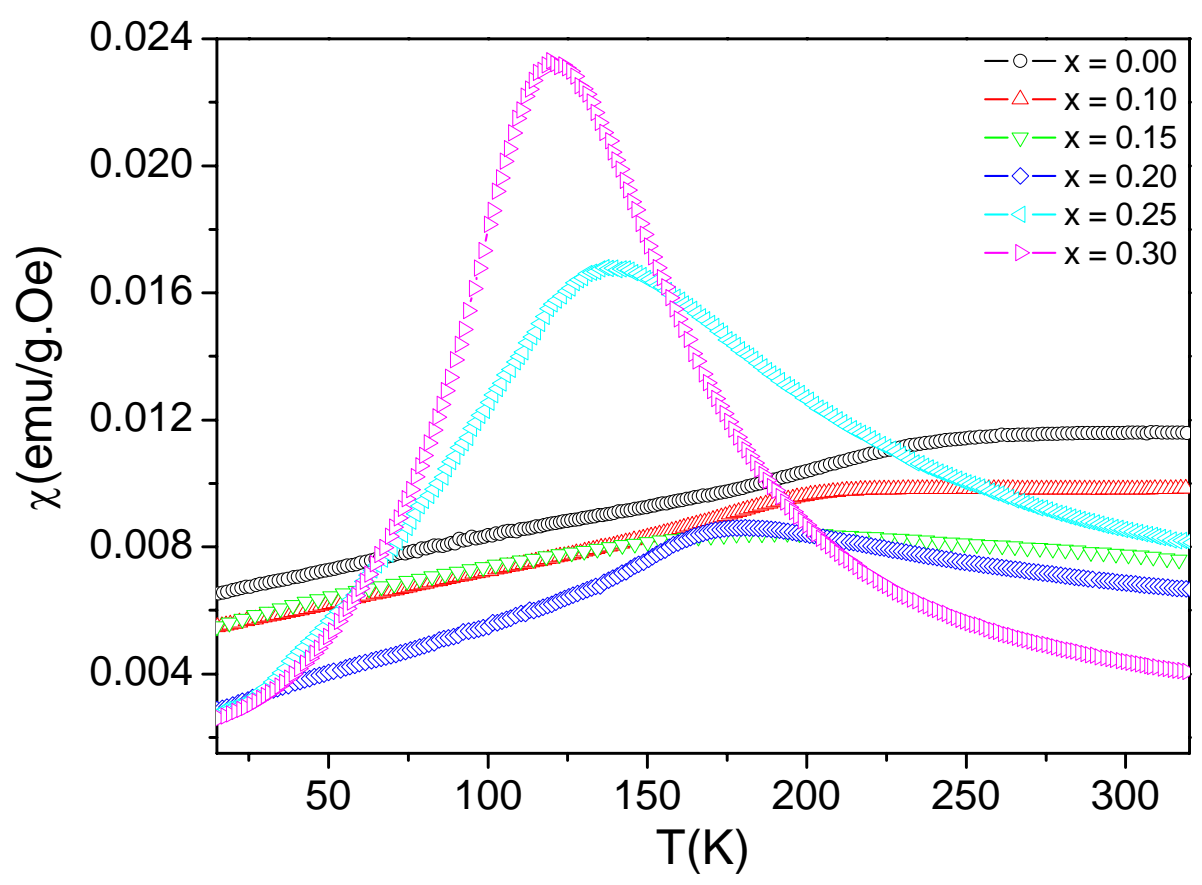

Figura 5.41: Susceptibilidad magnética en función de la temperatura.

\begin{tabular}{|c|c|c|c|c|c|c|}
\hline $\mathrm{x}$ & 0.00 & 0.10 & 0.15 & 0.20 & 0.25 & 0.30 \\
\hline$T_{\max }$ & $250_{5} \mathrm{~K}$ & $205_{4} \mathrm{~K}$ & $190_{4} \mathrm{~K}$ & $178_{2} \mathrm{~K}$ & $138_{2} \mathrm{~K}$ & $120_{1} \mathrm{~K}$ \\
\hline
\end{tabular}

Tabla 5.10: Temperatura del máximo de las curvas de susceptibilidad.

La evolución de $T_{\max }$ con x y $N^{*}{ }_{C u}$ se muestra en la figura 5.42. Allí se puede ver que existe una dependencia lineal de la temperatura de los máximos con x y $N^{*}{ }_{C u}$. Otro punto interesante para mencionar es que las temperaturas a las cuales se observan los máximos son próximas a las temperaturas en las que el campo magnético hiperfino comienza a incrementarse (flechas figura 5.38), esto indica que ambas técnicas están dando cuenta de la aparición del mismo orden magnético. De acuerdo a lo presentado en el Capítulo 3 se podría en principio, asociar las características de las curvas de susceptibilidad con un comportamiento de partículas superparamagnéticas con una gran distribución de tiempos de relajación; siendo en este caso la temperatura del máximo de la curva de susceptibilidad la temperatura de bloqueo del sistema. Esta temperatura de bloqueo depende del volumen medio de las partículas superparamagnéticas y, por lo tanto, un comportamiento como el observado en la figura 5.41 estaría indicando que existe una evolución del tamaño medio de las partículas con el contenido de $\mathrm{Cu}$. Dado que no se tiene 
evidencia experimental de este cambio de tamaño, esta hipótesis puede ser, en principio, descartada. Por otra parte, la constante de anisotropía del sistema también influye en la temperatura del máximo de la susceptibilidad. Para obtener una forma de curva similar a la observada en la figura 5.41 se debería tener una gran distribución de valores de la constante de anisotropía con un valor medio diferente para cada muestra. Los tipos de anisotropías más comunes son la anisotropía magnetocristalina, la anisotropía de forma y la anisotropía por tensiones. ${ }^{39} \mathrm{Si}$ bien los valores de la constante de anisotropía pueden ser distintos para cada una de las preparaciones debido a la concentración de $\mathrm{Cu}$, debido a que todas las muestras fueron preparadas en condiciones similares, es difícil pensar que éstas puedan tener valores de $K_{A}$ suficientemente diferentes entre sí para causar el efecto observado.

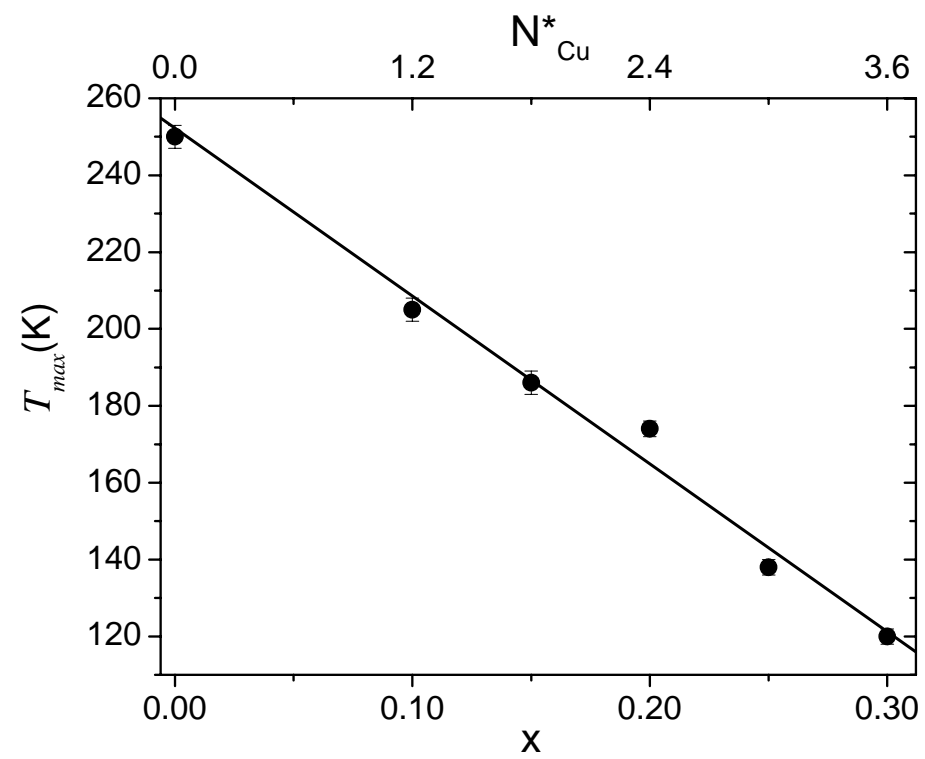

Figura 5.42: Variación de la temperatura del máximo de la susceptibilidad en función del contenido de $\mathrm{Cu}$ (abscisa inferior) y del número más probable de átomos de $\mathrm{Cu}$ primeros vecinos (abscisa superior).

Para descartar por completo la posibilidad de que el máximo presentado en las curvas de $\chi^{\prime}$ vs. $T$ provenga de un comportamiento de partículas superparamagnéticas es necesario estudiar la respuesta dinámica del mismo. Para ello se realizaron medidas de susceptibilidad para distintas frecuencias del campo AC. Como se mencionó en el Capítulo 3, la posición del máximo de la curva de susceptibilidad depende de la ventana temporal de medida que, en el caso de una medida de susceptibilidad AC, es inversamente proporcional a la frecuencia del campo excitante. Entonces, se espera que un sistema superparamagnético muestre una clara dependencia de la temperatura del máximo con dicha frecuencia (ver Ec. 3.7). Por otra parte, si se estuviese en presencia de una transición de fase, la temperatura del máximo sería independiente de las condiciones en las que se realice la medida. Una tercera posibilidad es que la variación observada sea muy pequeña, comportamiento asociado a los sistemas que presentan frustración magnética (vidrios de espín). ${ }^{40} \mathrm{~A}$ 
continuación se muestran en la figura 5.43 las de medidas de las muestras con $\mathrm{x}=0.20, \mathrm{x}=0.25 \mathrm{y}$ $\mathrm{x}=0.30$ para frecuencias $(\mathrm{v})$ entre $48 \mathrm{~Hz}$ y $10 \mathrm{kHz}$. No se realizaron medidas para las muestras con bajos contenidos de $\mathrm{Cu}$ ya que la cúspide no está bien definida, en parte, debido a la superposición con la fase ferromagnética $b c c-\mathrm{Fe}(\mathrm{MnCu})$, la cual posee una temperatura de orden muy superior a la máxima temperatura empleada en las medidas de susceptibilidad. En las curvas de la figura 5.43 se observa una leve variación de la temperatura del máximo de $\chi^{\prime}$ con la frecuencia del campo AC, la cual se desplaza hacia temperaturas mayores a medida que la frecuencia aumenta.
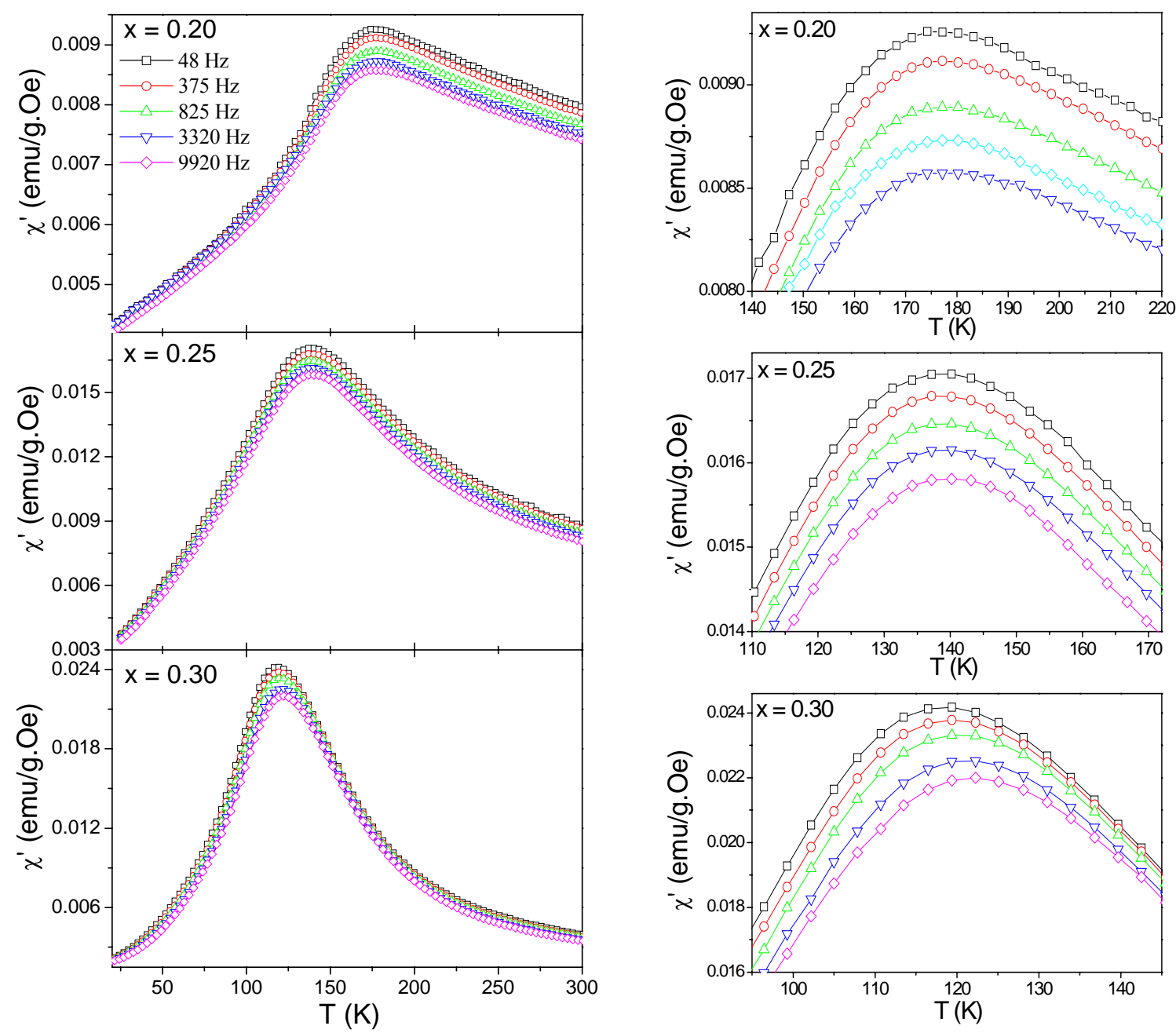

Figura 5.43: Variación de la susceptibilidad con la temperatura, para distintas frecuencias del campo de excitación. A la derecha se muestra la región del máximo ampliada.

La forma usual de cuantificar esta variación es determinar la variación de la temperatura del máximo por década de frecuencia, ${ }^{41}$ tal como lo expresa el parámetro $p$ : 


$$
p=\frac{\Delta T}{\bar{T} \Delta \log (v)}
$$

donde $\Delta T$ es la variación entre la temperatura de dos máximos medidos a dos frecuencias distintas, $\bar{T}$ la temperatura promedio entre dichos máximos y $\Delta \log (v)$ la diferencia entre los logaritmos de las frecuencias. En este caso, $p$ resulta un orden de magnitud más pequeño (ver Tabla 5.11) que el esperado para un material superparamagnético $(p \approx 0.3),{ }^{40}$ siendo el valor obtenido del orden del encontrando en sistemas con frustración magnética, ${ }^{40}$ por esto es posible asociar la relajación observada con un material que presenta un comportamiento tipo vidrio de espín.

\begin{tabular}{|c|c|c|c|c|c|}
\hline $\mathrm{x}$ & $T_{\max }(\mathrm{K}) \mathrm{a} 825 \mathrm{~Hz}$ & $p$ & $T_{0}(\mathrm{~K})$ & $\tau_{0}(\mathrm{~s})$ & $\mathrm{z} v$ \\
\hline 0.20 & $178_{2}$ & 0.003 & $175_{2}$ & $10_{2} \times 10^{-13}$ & $5.2_{2}$ \\
\hline 0.25 & $138_{2}$ & 0.005 & $135_{2}$ & $6_{1} \times 10^{-13}$ & $6.1_{2}$ \\
\hline 0.30 & $120_{1}$ & 0.012 & $112_{1}$ & $6_{1} \times 10^{-13}$ & $6.9_{1}$ \\
\hline
\end{tabular}

Tabla 5.11: Valores de la temperatura del máximo de $\chi^{\prime}\left(T_{\max }\right)$, de la sensibilidad a la frecuencia $(p)$, del parámetro de Vogel-Fulcher $\left(T_{0}\right)$ y los parámetros de la ley de exponentes críticos $\left(\tau_{0} \mathrm{y} z v\right)$.

Seguidamente, se realizó un estudio para determinar si el sistema de partículas era interactuante o no. Como se describió en el Capítulo 3, un sistema de partículas no interactuantes tendrá una relación entre el tiempo de relajación y su temperatura de bloqueo, que seguirá un comportamiento del tipo Arrhenius, como el descripto por la ecuación 3.2. Empleando este modelo no se obtiene una buena reproducción de los datos experimentales y los valores de los parámetros encontrados no poseen significado físico. Por este motivo, se propuso reproducir el comportamiento de $T_{\max }$ usando la ley de Vogel-Fulcher: ${ }^{42}$

$$
\tau=\tau_{0} \exp \left[\frac{K_{A} V}{k_{B}\left(T_{\max }-T_{0}\right)}\right]
$$

la cual incorpora un parámetro fenomenológico $T_{0}$ que se considera como una medida del grado de interacción del sistema. Los resultados del ajuste empleando la ecuación anterior son presentados en la figura 5.44. Se puede apreciar que el modelo reproduce satisfactoriamente el comportamiento de los datos experimentales, indicando que el sistema posee un grado de interacción entre sus partículas el cual no puede ser ignorado. 

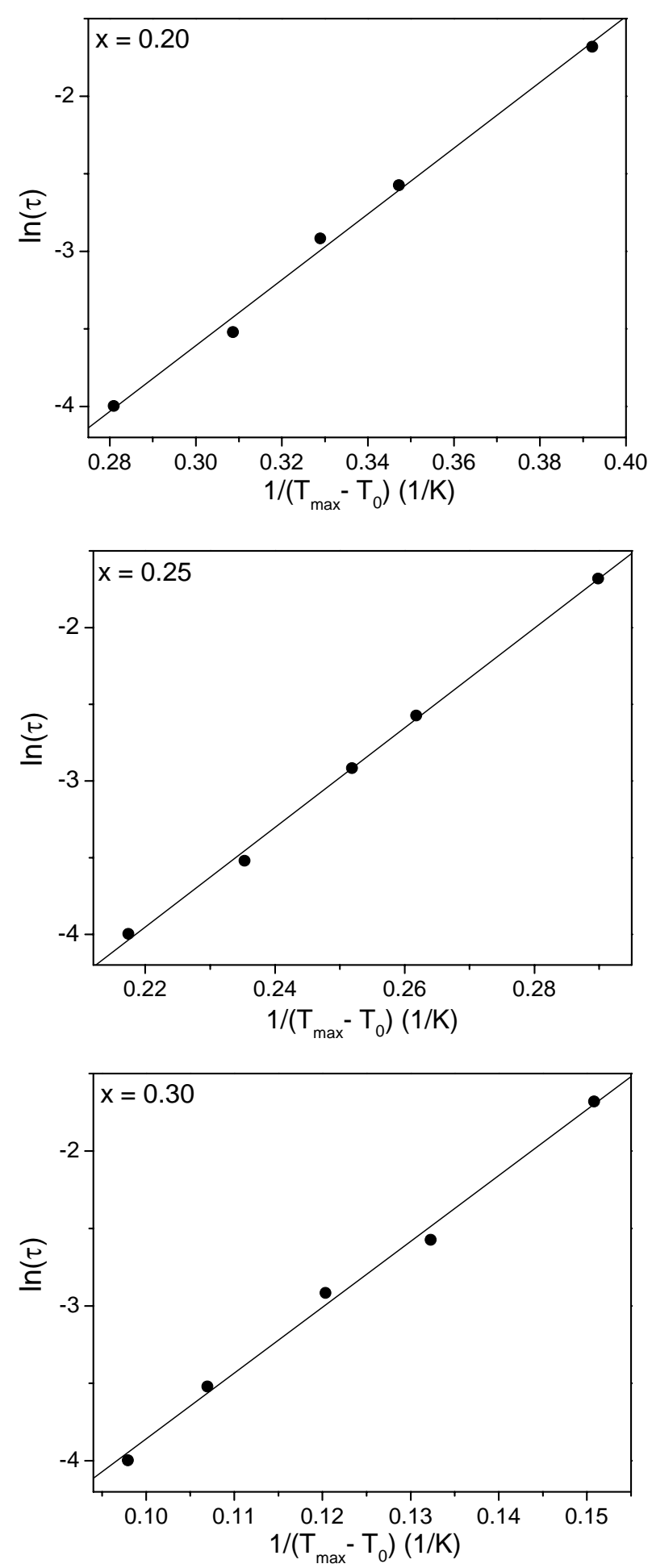

Figura 5.44: Variación del $\ln (\tau)$ en función de $1 /\left(\mathrm{T}_{\max }-\mathrm{T}_{0}\right)$ para las muestras $\mathrm{x}=0.20,0.25$ y 0.30 . La línea sólida es el ajuste lineal obtenido. 
Finalmente un análisis de acuerdo a una ley de exponentes críticos es la herramienta más empleada para discernir el carácter magnético del sistema. ${ }^{43}$ De acuerdo a como se presentó en el Capítulo 3, la variación de $T_{\max }$ con el tiempo característico de medida se obtiene en el marco de un fenómeno crítico, empleando la siguiente expresión:

$$
\tau=\tau_{0}\left[\frac{T_{\max }-T_{c}}{T_{c}}\right]^{-z v}
$$

En la figura 5.45 se presentan los resultados de los ajustes empleando la ecuación anterior para las muestras con $x \geq 0.20$. Los resultados obtenidos (ver Tabla 5.11) muestran que los valores del exponente $z v$ varían dentro del rango 5.2 a 6.9 , mientras que los valores correspondientes para $\tau_{0}$ se encuentran entre $10^{-12} \mathrm{~s}$ a $10^{-13} \mathrm{~s}$, estando en todos los casos dentro de lo esperado para sistemas con comportamiento tipo vidrio de espín. ${ }^{44}$ 

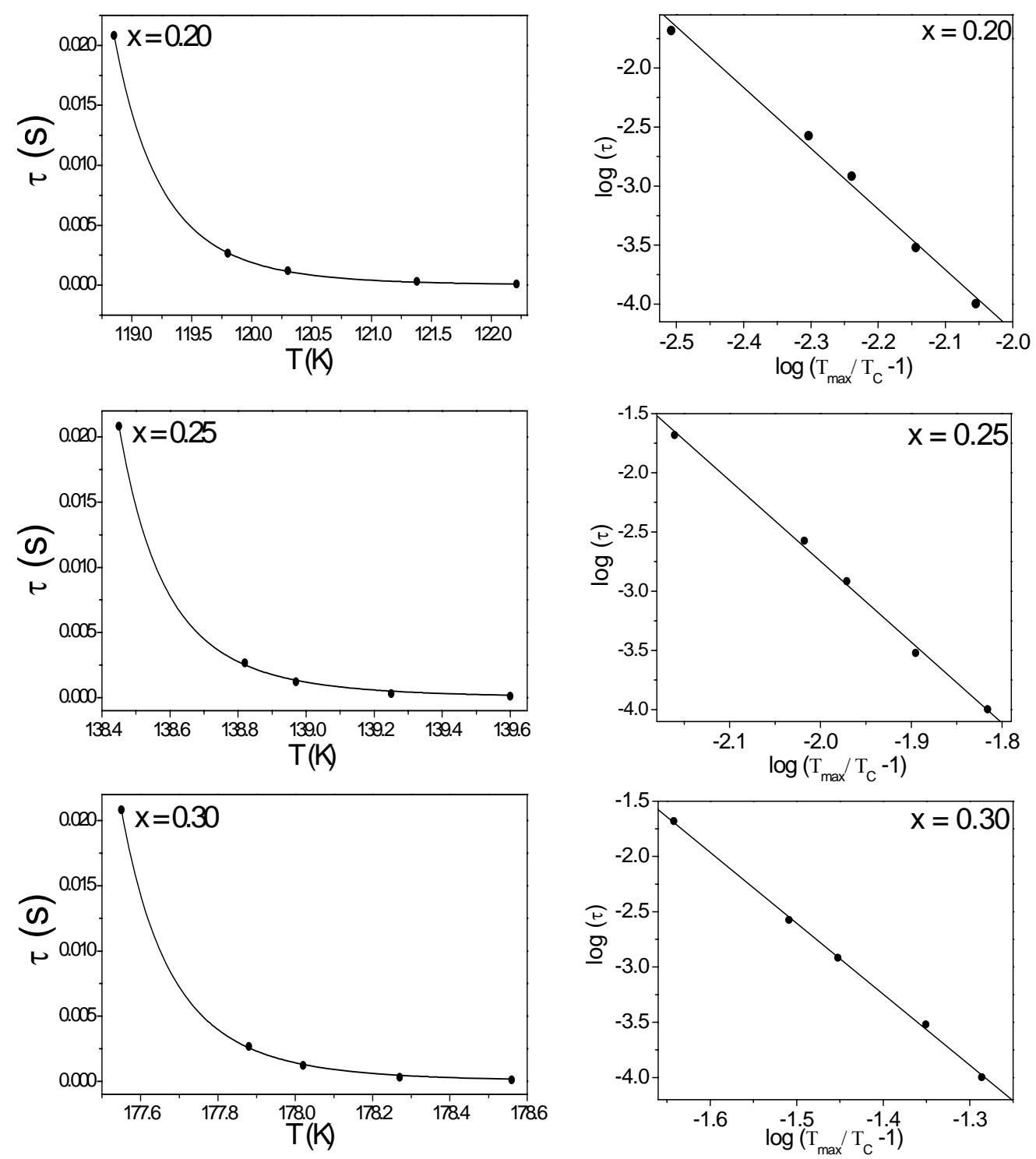

Figura 5.45: Variación del tiempo característico de medida en función de la temperatura del máximo de la susceptibilidad (izq.) junto con el ajuste según la Ec. 5.9 (línea sólida). $\log (\tau)$ en función de $\log \left[\left(T_{\max } / T_{c}-\right.\right.$ 1)] (der.) junto al ajuste lineal a los datos (línea sólida).

Otra característica de los vidrios de espín es la marcada variación de la susceptibilidad AC con campos magnéticos estáticos débiles en las cercanías de la temperatura del máximo. ${ }^{45}$ Entonces, se realizaron en la muestra con $\mathrm{x}=0.30$ una serie de medidas de $\chi^{\prime}$ vs. $T$ aplicando diferentes campos magnéticos externos DC $\left(H_{D C}\right)$ de intensidades 5 Oe, 10 Oe y 20 Oe. Los resultados obtenidos son mostrados en la figura 5.46. Como se aprecia, el campo magnético DC produce una disminución en la intensidad de la curva en la región cercana al máximo, mientras que las curvas coinciden en otras regiones. Además, se nota que la presencia del campo DC desplaza la posición del máximo a temperaturas más bajas. Estas características son comúnmente asociadas en 
la bibliografía ${ }^{46}$ con condiciones necesarias para que el sistema sea considerado como un vidrio de espín.

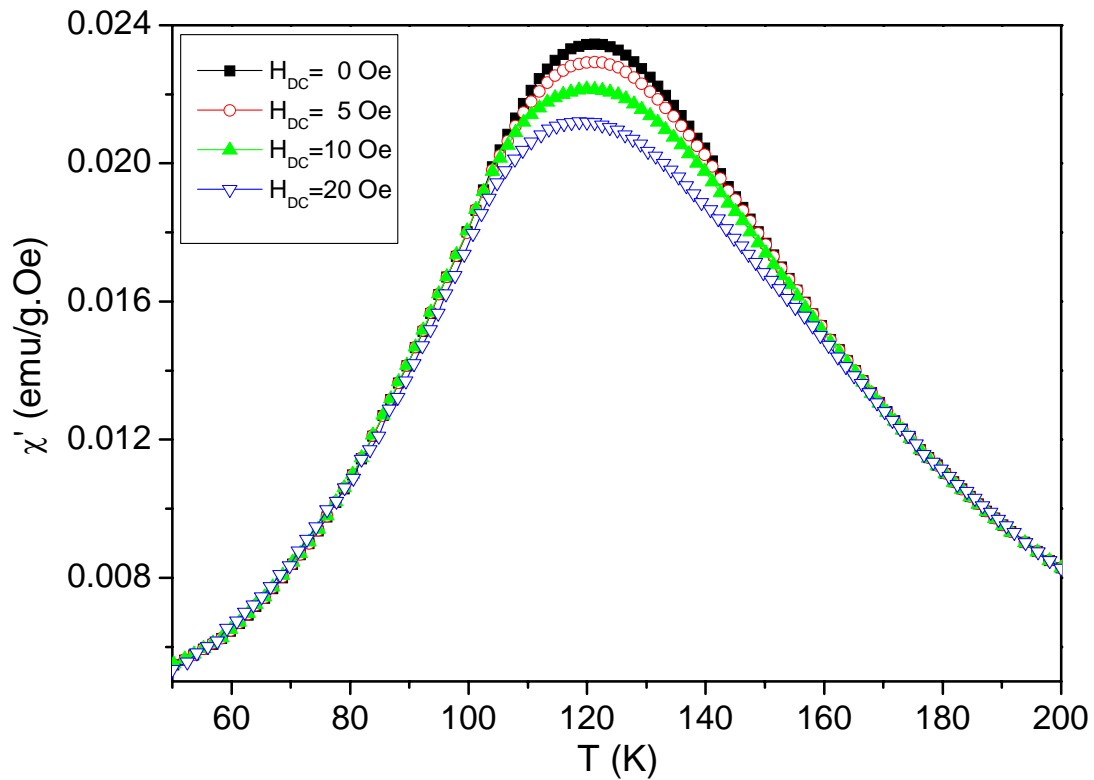

Figura 5.46: Variación de la componente en fase de la susceptibilidad AC en la muestra $\mathrm{x}=0.30$ en presencia de un campo magnético estático $\mathrm{H}_{\mathrm{DC}}$.

Por otra parte, la dependencia con la temperatura de la susceptibilidad no lineal, definida en el Capítulo 3, es empleada para estudiar una posible transición de fase a $T_{\max }$. En vidrios de espín canónicos se espera encontrar una divergencia de la susceptibilidad no lineal a $T_{\max }$ producto de la divergencia de la distancia de correlación. Para estudiar este comportamiento se propone un desarrollo de la magnetización $M$ en términos de $\chi_{0} H_{D C}$. Este desarrollo se hace en términos de $\chi_{0} H_{D C}$ en lugar de sólo $H_{D C}$ a fin de evitar una sobreestimación de la dependencia del término no lineal con la temperatura, producto de un posible comportamiento diferente a Curie-Weiss en las cercanías de $T_{\max }$. De esta forma, se expresa la magnetización como:

$$
M \approx \chi_{0} H_{D C}-a_{3}\left(\chi_{0} H_{D C}\right)^{3}
$$

donde el coeficiente $a_{3}\left(a_{3}=\left(\chi^{\prime}-\chi_{0}\right) /\left(\chi_{0}^{3} H_{D C}^{2}\right)\right)$ está asociado al comportamiento no lineal del sistema y $\chi_{0}$ corresponde a la medida con $H_{D C}=0$ Oe. La evolución del coeficiente $a_{3}$ con la temperatura es mostrada en la figura 5.47. Las tres curvas muestran un mínimo en las cercanías de $T_{\max }$, tal como en un material vidrio de espín, ${ }^{47}$ aunque en estas curvas la divergencia no es tan acentuada. Por esta razón, no es posible afirmar la presencia de una transición de fase. 


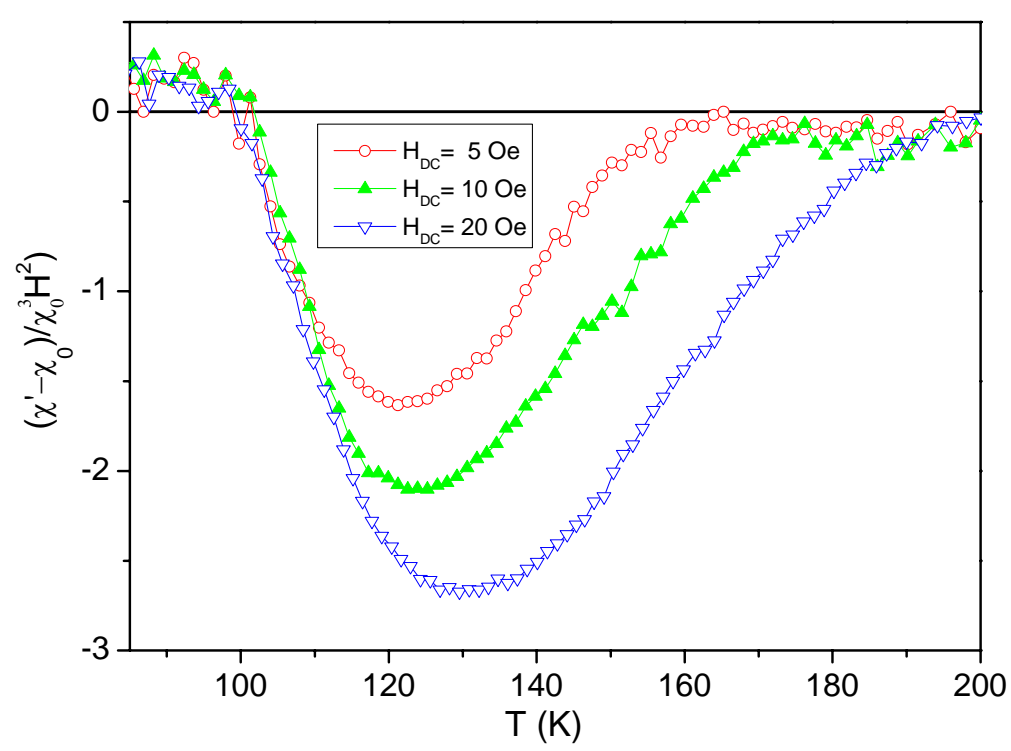

Figura 5.47: Variación del coeficiente $a_{3}$ de la susceptibilidad no lineal con la temperatura.

Se ha observado que, en sistemas en donde las interacciones entre sus partículas son fuertes, el régimen a bajas temperaturas es cercano al presentado por vidrios de espín canónicos. ${ }^{48}$ En sistema nanoaglomerados, como las estudiadas en esta Tesis, la yuxtaposición de las partículas puede conducir a interacciones de intercambio entre átomos de la superficie de las partículas, llevando a un comportamiento tipo vidrio de espín. A medida que la temperatura se incrementa, alcanzando la temperatura de congelamiento de los bordes, las partículas se desacoplan y la curva de susceptibilidad alcanza su máximo, como es observado en las muestras con altos contenidos de $\mathrm{Cu}$ (figura 5.41). Finalmente, la existencia de interacciones dipolares o del tipo RKKY no pueden ser descartadas ya que ambas son posibles en este tipo de muestras. La presencia de las mismas puede conducir a una competencia entre interacciones ferro-antiferromagneticas, las cuales, junto con el desorden atómico producto de la técnica de preparación, producen un comportamiento colectivo de los momentos magnéticos del tipo vidrio de espín. 


\section{Ciclos de histéresis}

En la figura 5.48 se presentan los resultados de los ciclos de histéresis medidos a temperatura ambiente para las aleaciones estudiadas. Los ciclos presentan un comportamiento histerético para todas las concentraciones. La magnitud del campo coercitivo disminuye desde $0.022 \mathrm{~T}$ para la muestra $\sin \mathrm{Cu}$ y hasta $0.004 \mathrm{~T}$, para la muestra con $\mathrm{x}=0.30$. Por otra parte, la magnetización a altos campos magnéticos también disminuye a medida que aumenta el contenido de $\mathrm{Cu}$. Para las muestras con $\mathrm{x}=0.00, \mathrm{x}=0.10 \mathrm{y} \mathrm{x}=0.15$ el alto valor de la magnetización está asociado a la presencia de la fase $b c c-\mathrm{Fe}(\mathrm{Mn}, \mathrm{Cu})$ observada tanto en los difractogramas de mayor concentración de $\mathrm{Cu}$ como en las medidas Mössbauer.
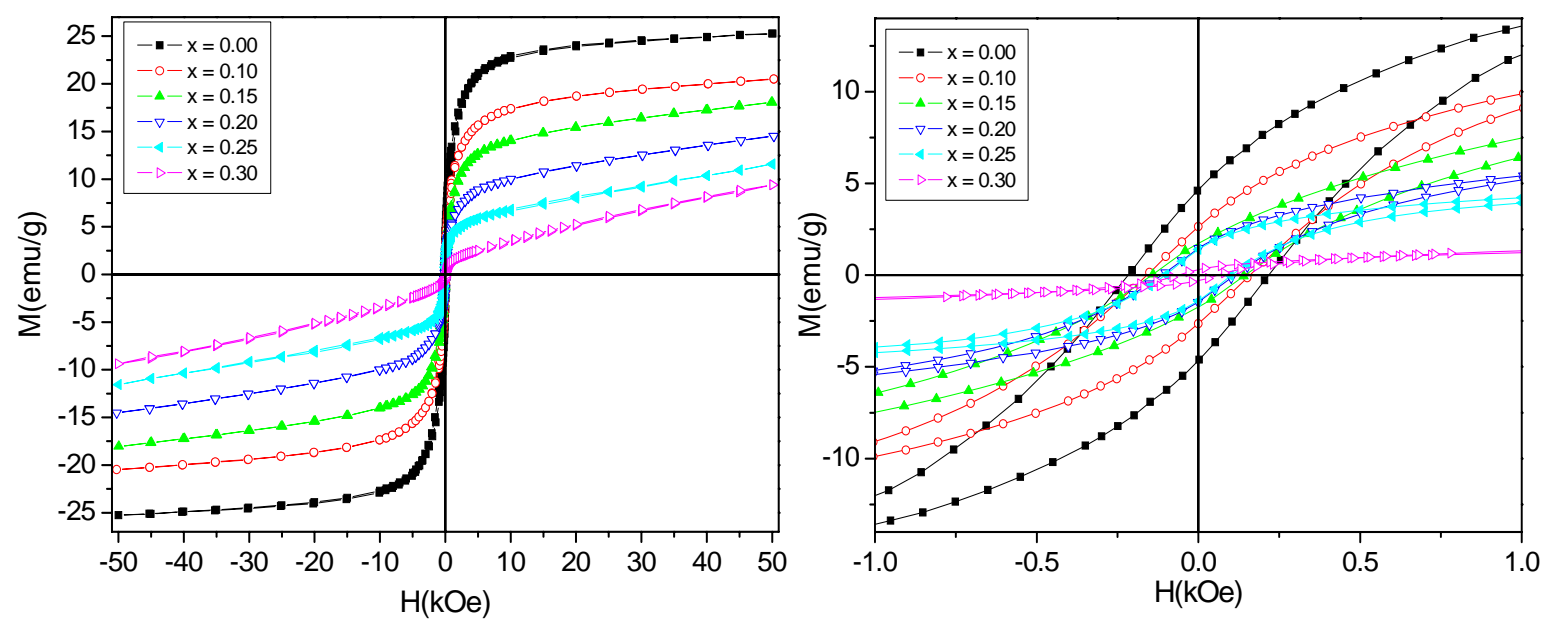

Figura 5.48: Ciclos de histéresis a $300 \mathrm{~K}$ (izq.). Región central de los ciclos (der.).

Para la muestra con $\mathrm{x}=0.00$, la magnetización presenta un comportamiento muy cercano a la saturación pero, siendo estrictos, nunca se alcanza dicho estado aún para campos magnéticos de $5 \mathrm{~T}$. A medida que la concentración de $\mathrm{Cu}$ aumenta, la magnetización presenta un comportamiento casi lineal para altos campos, siendo esto más evidente para las muestras con $\mathrm{x} \geq 0.20$.

Para profundizar en el estudio del sistema, se realizaron medidas de los ciclos de histéresis a bajas temperaturas. A continuación, en las figuras 5.49 a 5.54, se muestran los resultados de los ciclos de histéresis a distintas temperaturas. Para facilitar la comparación se han incluido los resultados a temperatura ambiente. 

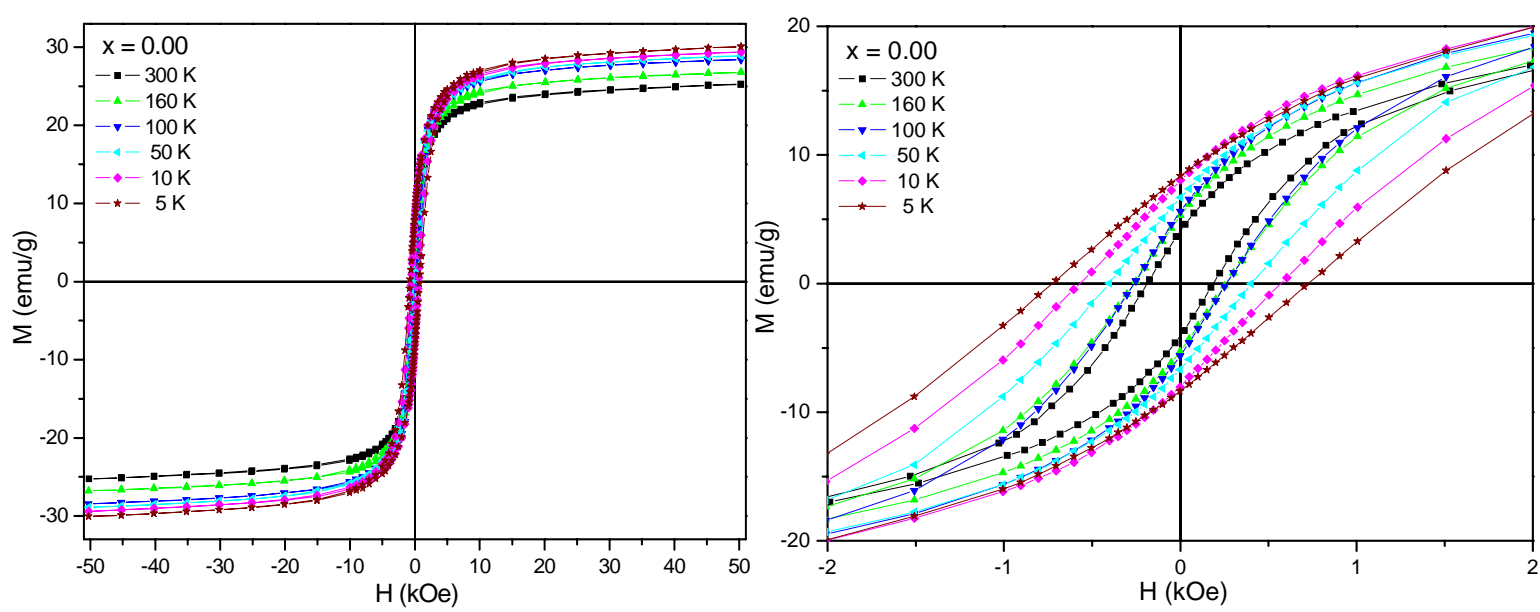

Figura 5.49: Medidas de histéresis entre $300 \mathrm{~K}$ y $5 \mathrm{~K}$ para la muestra con $\mathrm{x}=0.00$ (izq.). Región central de los ciclos (der.).
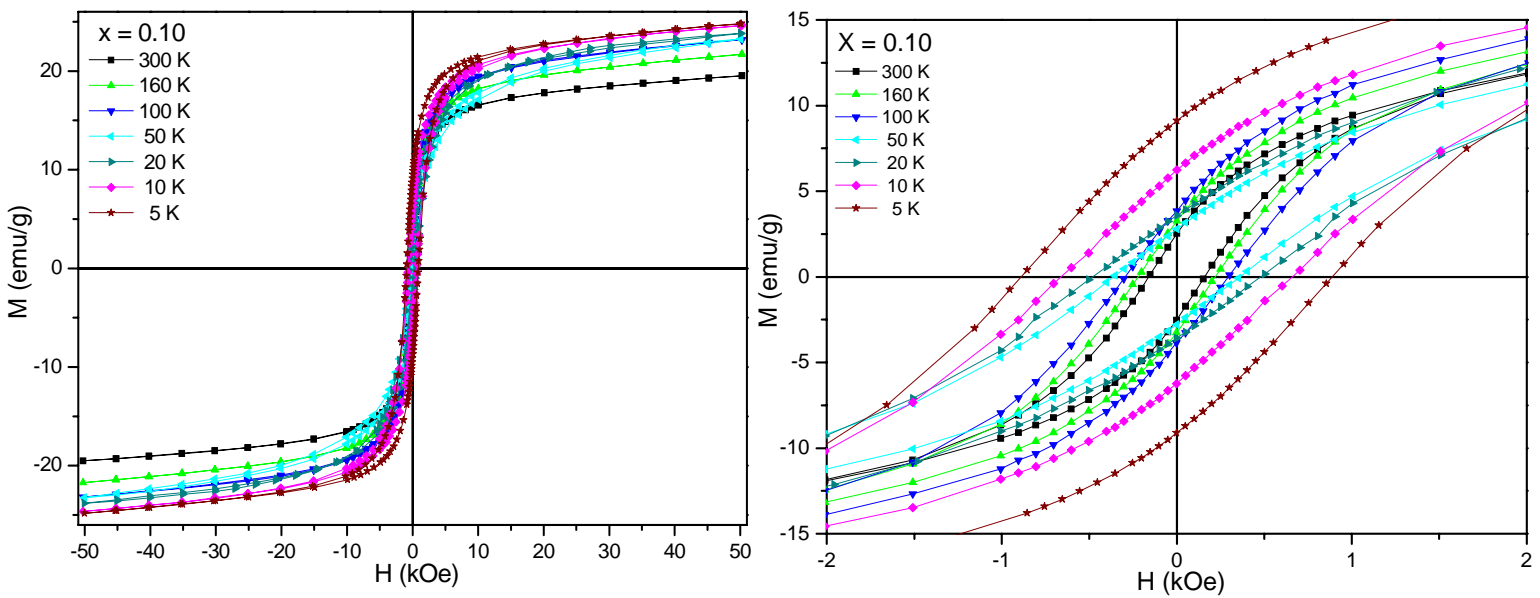

Figura 5.50: Medidas de histéresis entre $300 \mathrm{~K}$ y $5 \mathrm{~K}$ para la muestra con $\mathrm{x}=0.10$ (izq.). Región central de los ciclos (der.). 

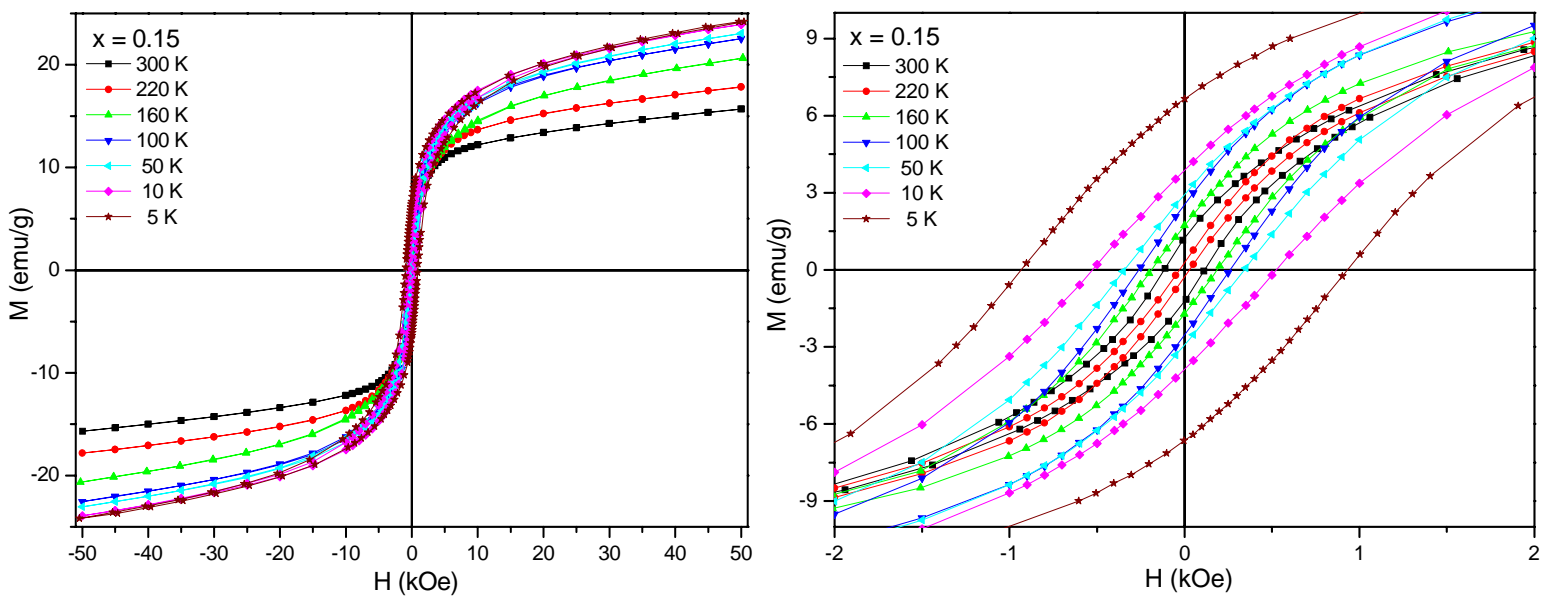

Figura 5.51: Medidas de histéresis entre $300 \mathrm{~K}$ y $5 \mathrm{~K}$ para la muestra con $\mathrm{x}=0.15$ (izq.). Región central de los ciclos (der.).
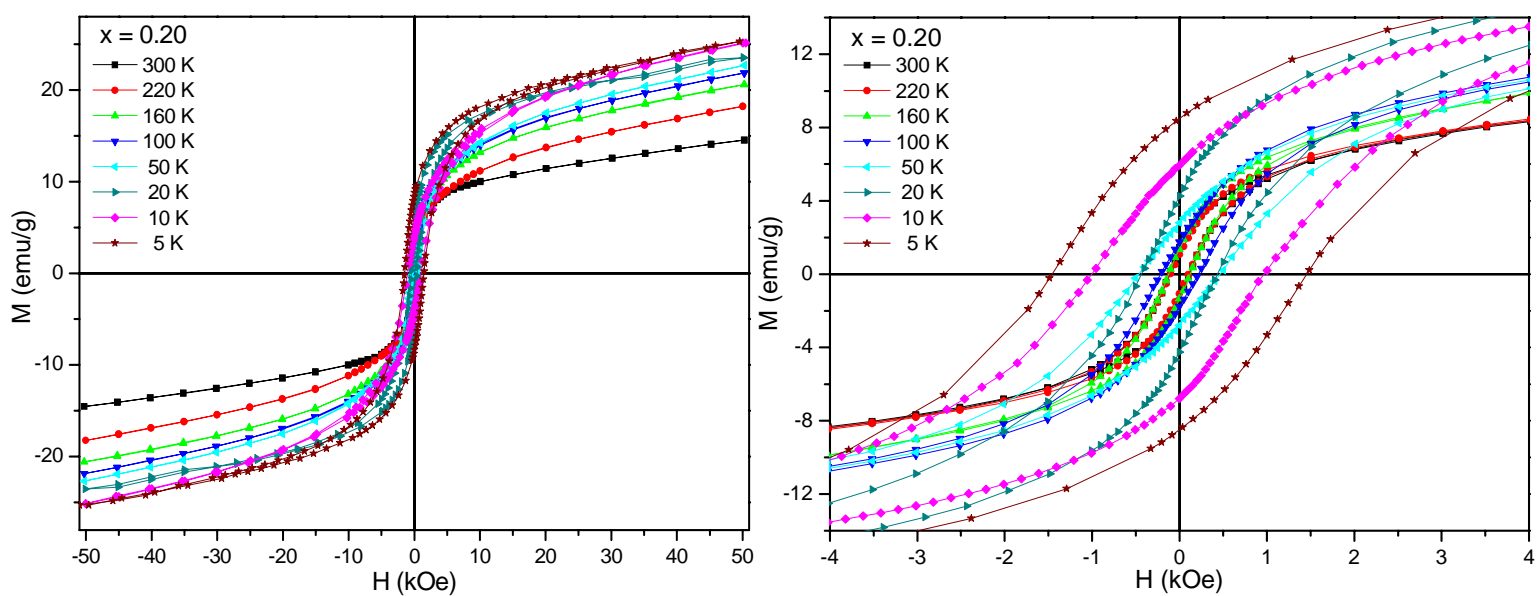

Figura 5.52: Medidas de histéresis entre $300 \mathrm{~K}$ y $5 \mathrm{~K}$ para la muestra con $\mathrm{x}=0.20$ (izq.). Región central de los ciclos (der.). 

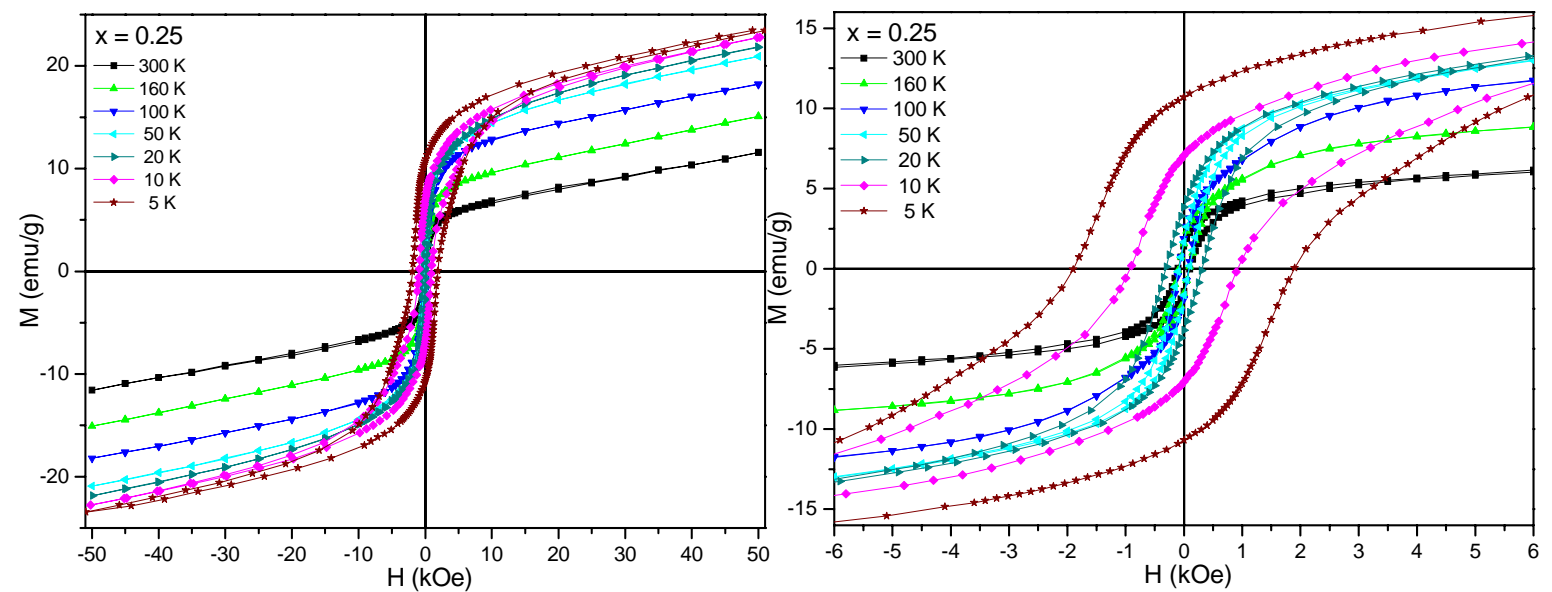

Figura 5.53: Medidas de histéresis entre $300 \mathrm{~K}$ y $5 \mathrm{~K}$ para la muestra con $\mathrm{x}=0.25$ (izq.). Región central de los ciclos (der.).
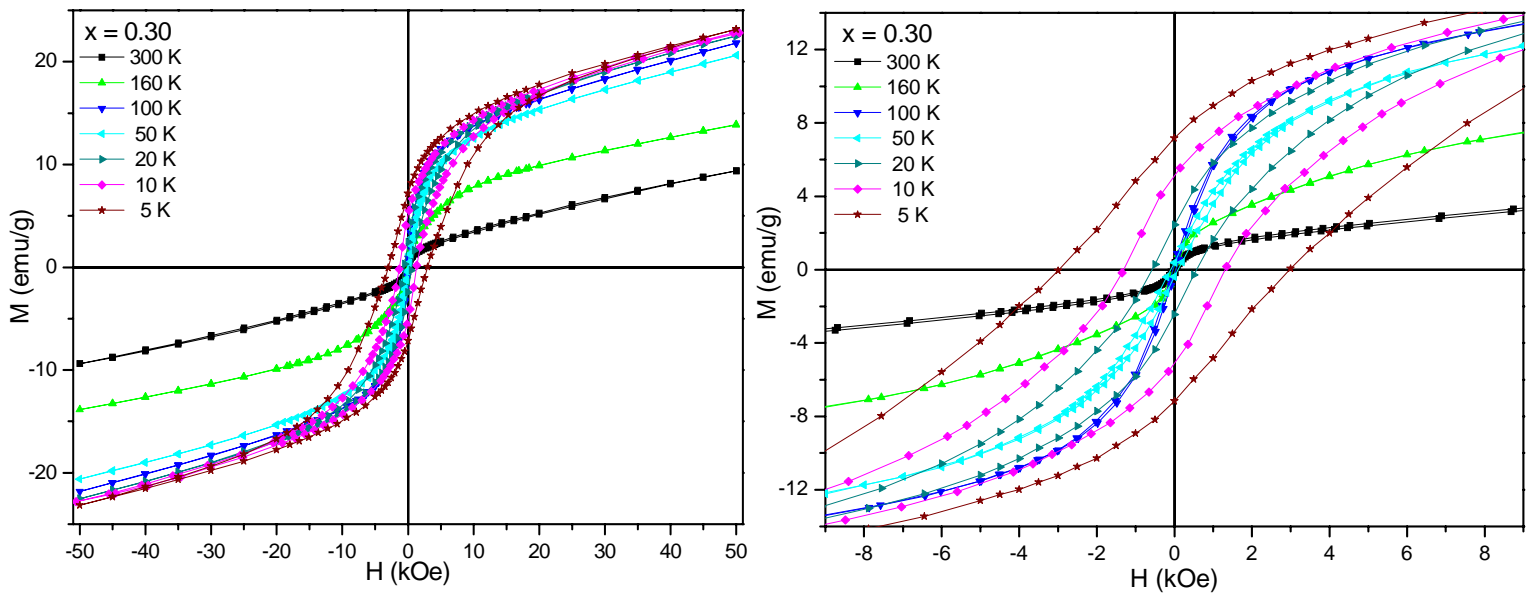

Figura 5.54: Medidas de los ciclos de histéresis entre $300 \mathrm{~K}$ y $5 \mathrm{~K}$ para la muestra con $\mathrm{x}=0.30$ (izq.). Región central de los ciclos (der.).

Las características que presentan las medidas con la variación de la temperatura son similares para las distintas concentraciones. Así, se puede mencionar el aumento del campo coercitivo y de la magnetización a $5 \mathrm{~T}$ con la disminución de la temperatura. También se observa que la pendiente de la curva de magnetización para altos campos no es afectada por el cambio de temperatura, resultando exclusivamente dependiente de la cantidad de $\mathrm{Cu}$. Las medidas realizadas a la temperatura más baja presentan en su región central una curvatura compleja, siendo esto un claro 
indicio de la presencia de más de una fase magnética. Una forma sencilla de estudiar este comportamiento es por medio de la derivada de los ciclos respecto al campo magnético. En el caso de un material con sólo una fase magnética ordenada, la derivada debería mostrar dos máximos, los cuales coinciden con los cruces por cero de la curva M vs. H, o sea, los campos coercitivos del ciclo a izquierda y derecha. A continuación se muestran los resultados de realizar la derivada de los lazos de histéresis con respecto al campo magnético. ${ }^{i}$ Como se ve en la figura 5.55, el máximo se desplaza hacia la izquierda reflejando, en valor absoluto, el aumento del campo coercitivo observado en las figuras 5.49 a 5.54. Este comportamiento está presente para todas las concentraciones. La característica más interesante que muestran las derivadas es el ensanchamiento que presentan a medida que disminuye la temperatura y la tendencia a ser asimétricas, lo cual es más evidente para las mayores concentraciones de $\mathrm{Cu}$. Este comportamiento es indicativo de la presencia de dos fases magnéticamente ordenadas, las cuales poseen campos coercitivos distintos.
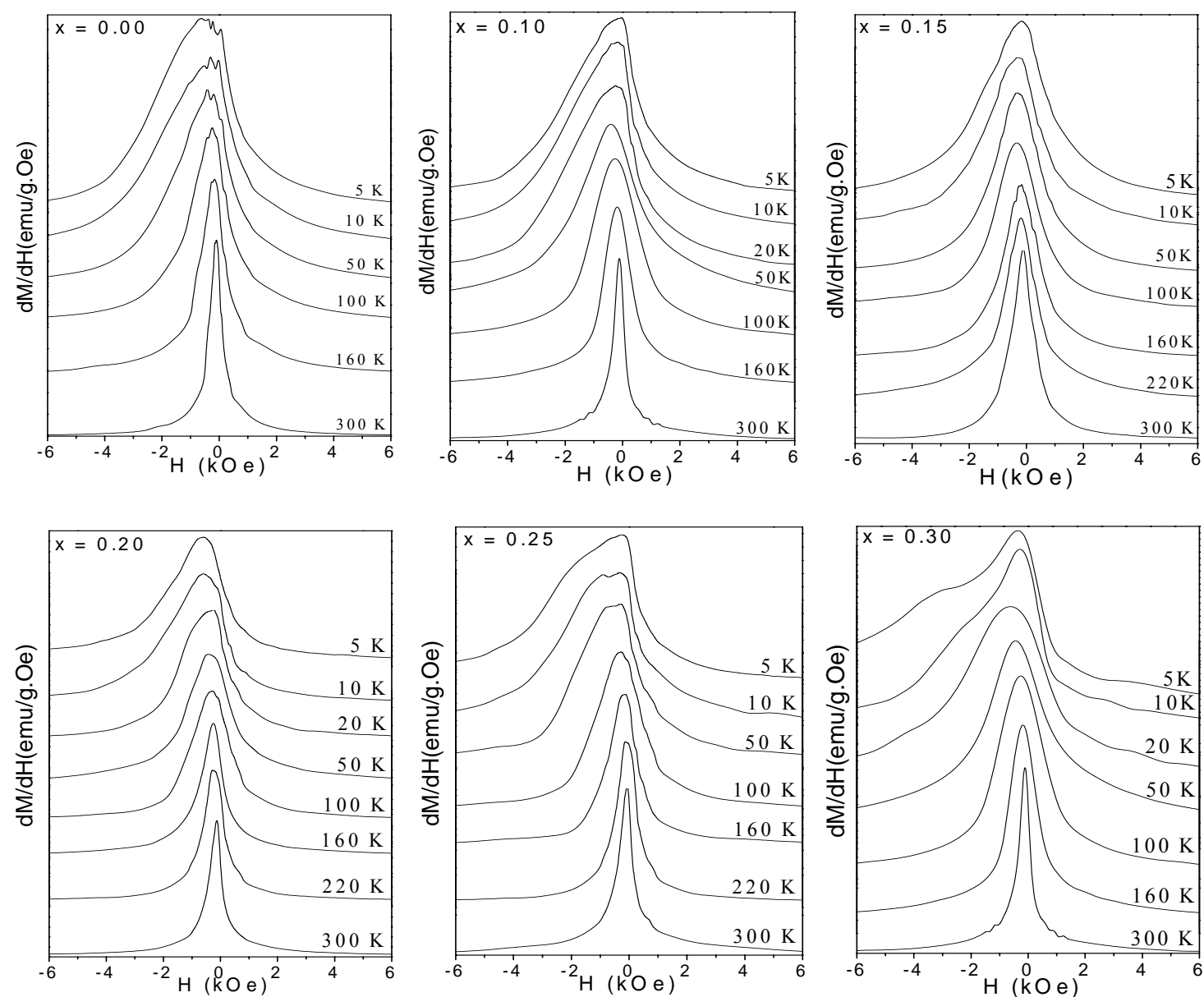

Figura 5.55: Derivadas de la rama izquierda de los ciclos de histéresis para distintas temperaturas.

\footnotetext{
${ }^{i}$ Se presenta sólo la derivada de la rama izquierda de los ciclos para una mayor claridad. La rama derecha presenta un comportamiento similar en todos los casos.
} 
Debido al comportamiento observado en las derivadas, es indudable que una nueva componente magnética ordenada aparece a bajas temperaturas. Es por esto que para la reproducción de estos ciclos se utilizó el siguiente modelo: para un ciclo medido a una temperatura superior a $T_{\max }$, se empleó la ecuación 4.10 , en la cual se contemplan una contribución ferromagnética, una curva de Langevin y una componente lineal con el campo; mientras que, para ciclos medidos a temperaturas inferiores a $T_{\max }$ la función empleada está descripta por la ecuación 4.11, donde la curva de Langevin se reemplazó por otra interacción ferromagnética, la cual estará caracterizada por los parámetros $\mathrm{Ms}_{2}$ y $\mathrm{Hc}_{2}$. A continuación, en las figuras 5.56 a 5.61 se muestran los ajustes de los ciclos de histéresis con las componentes empleadas para la reproducción de los mismos. 


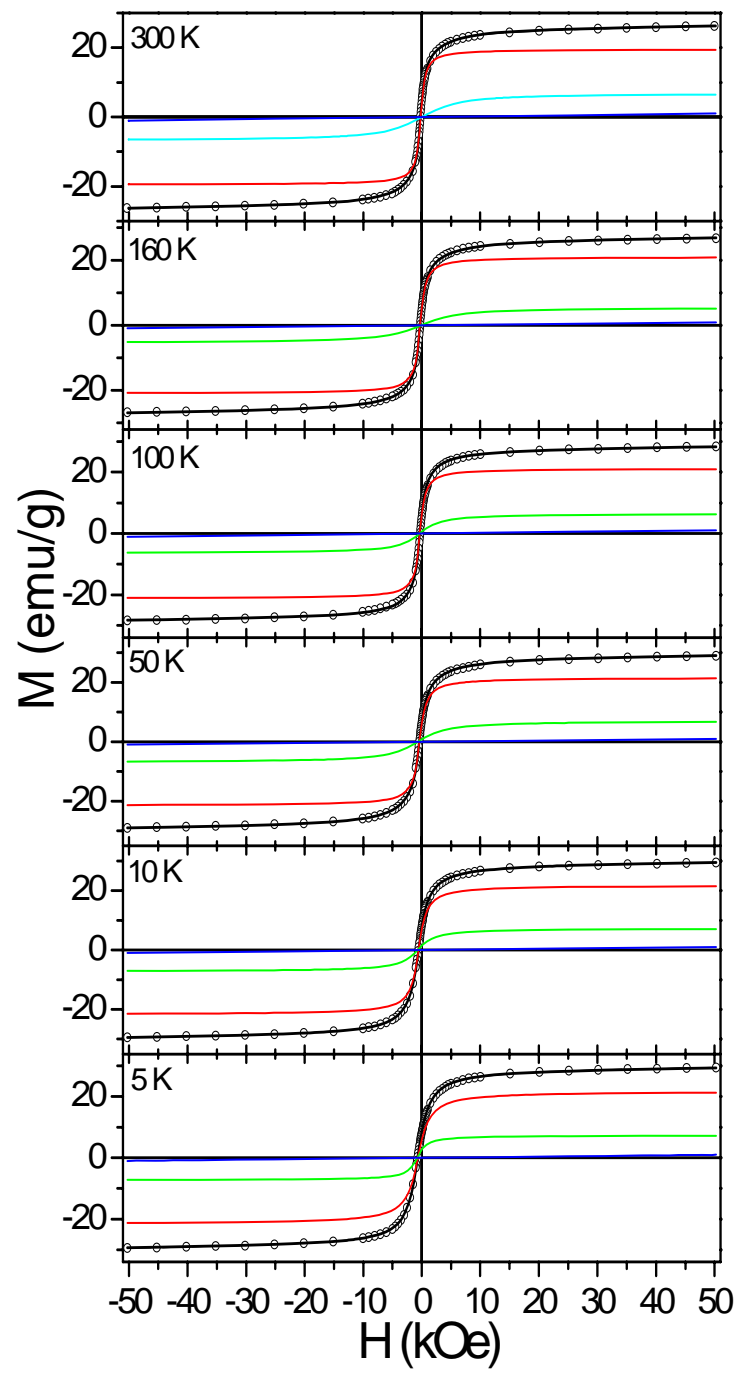

Figura 5.56: Resultados de los ajustes de los ciclos de histéresis medidos a bajas temperaturas para la muestra con $\mathrm{x}=0.00$. Ajuste propuesto (línea negra) y las distintas contribuciones al mismo: ferromagnética 1 (línea roja), ferromagnéticas 2 (línea verde), Langevin (línea celeste) y lineal (línea azul).

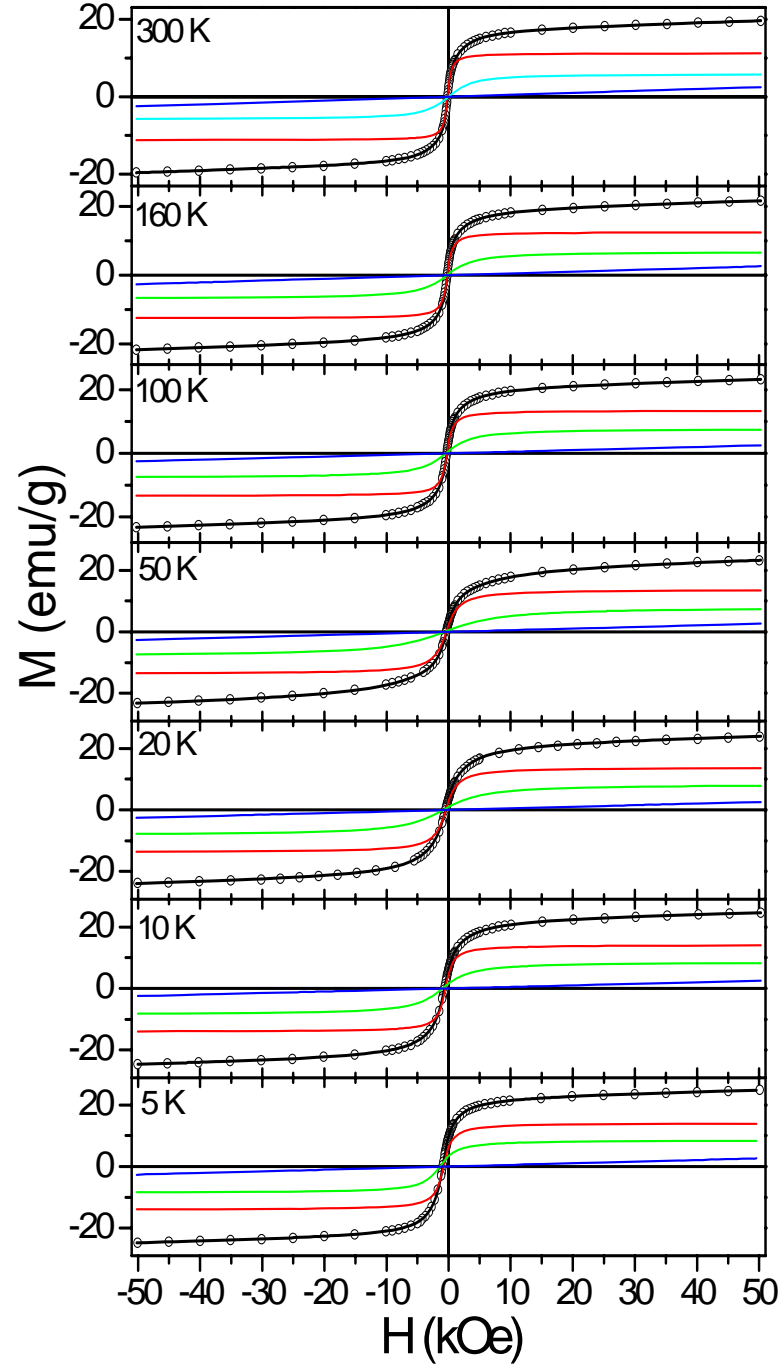

Figura 5.57: Resultados de los ajustes de los ciclos de histéresis medidos a bajas temperaturas para la muestra con $\mathrm{x}=0.10$. Ajuste propuesto (línea negra) y las distintas contribuciones al mismo: ferromagnética 1 (línea roja), ferromagnéticas 2 (línea verde), Langevin (línea celeste) y lineal (línea azul). 


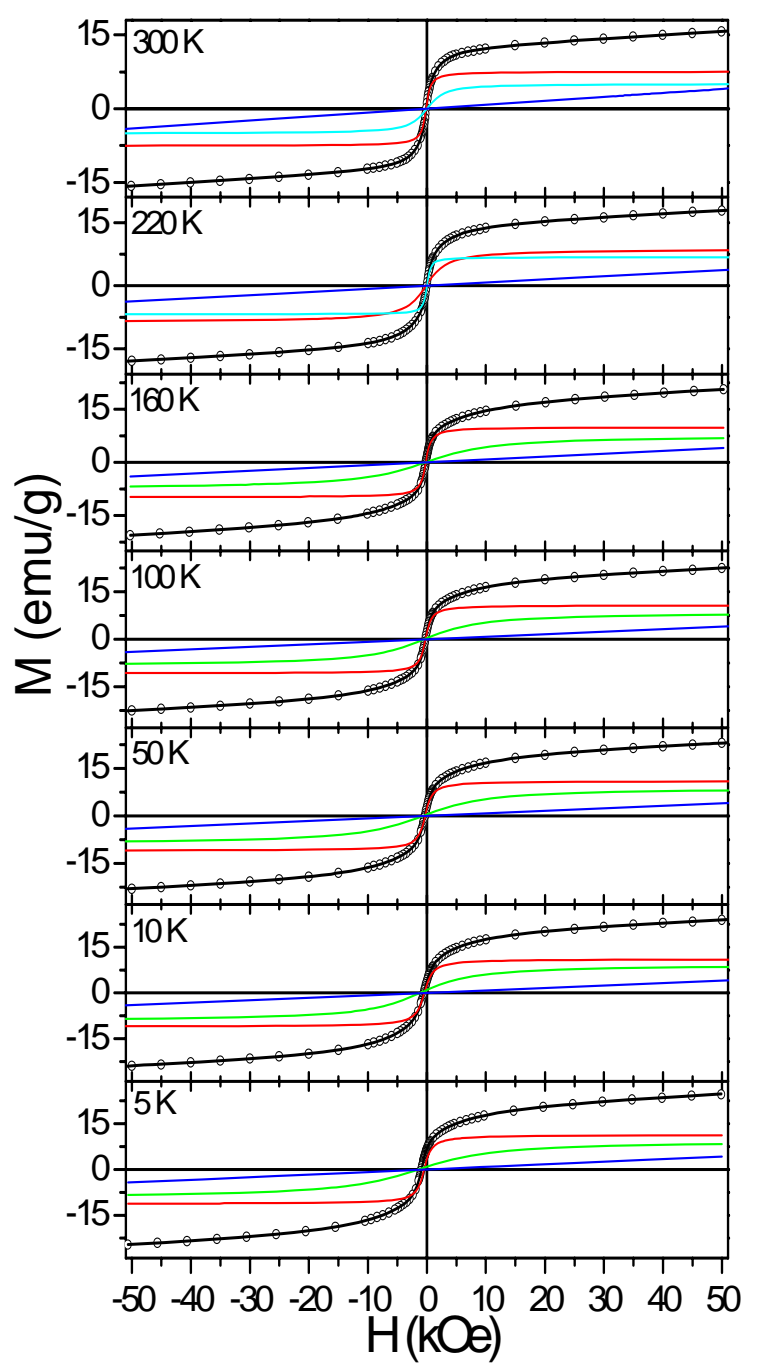

Figura 5.58: Resultados de los ajustes de los ciclos de histéresis medidos a bajas temperaturas para la muestra con $\mathrm{x}=0.15$. Ajuste propuesto (línea negra) y las distintas contribuciones al mismo: ferromagnética 1 (línea roja), ferromagnéticas 2 (línea verde), Langevin (línea celeste) y lineal (línea azul).

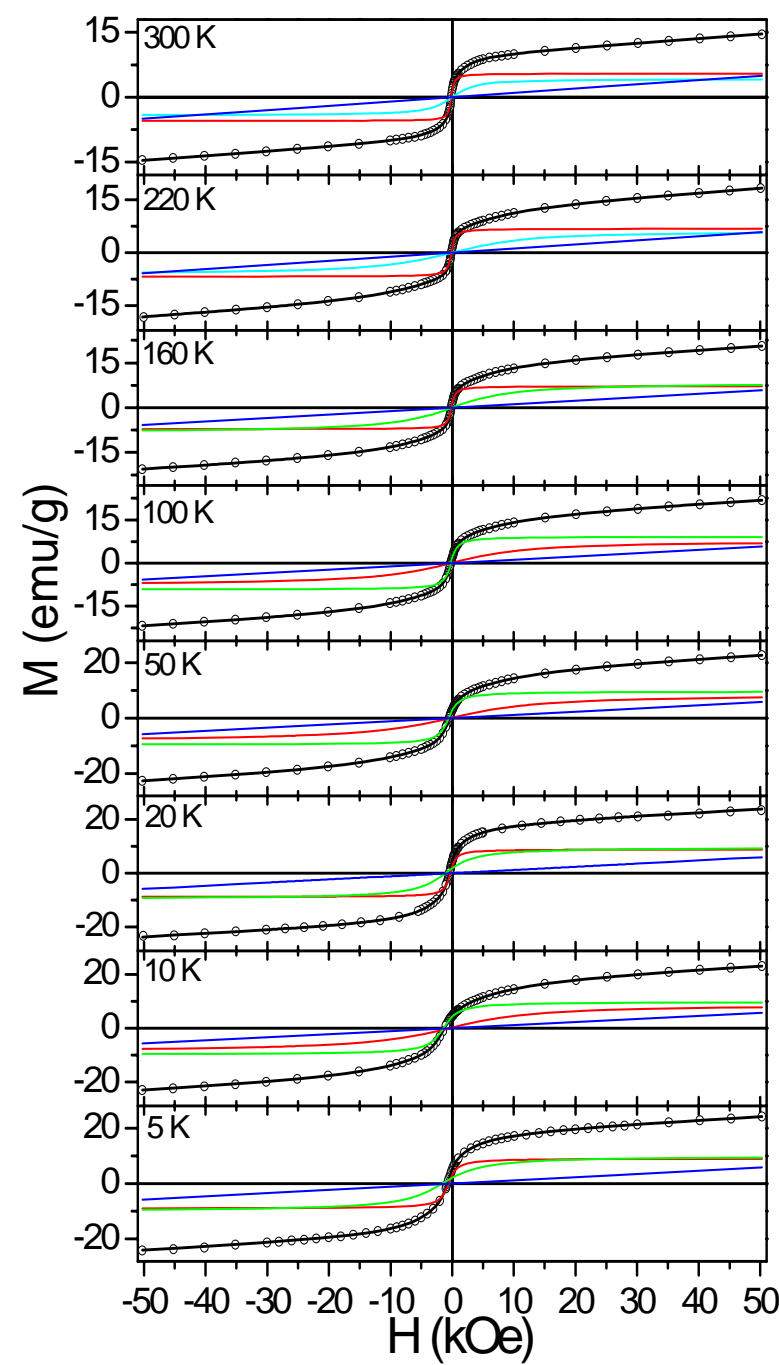

Figura 5.59: Resultados de los ajustes de los ciclos de histéresis medidos a bajas temperaturas para la muestra con $\mathrm{x}=0.20$. Ajuste propuesto (línea negra) y las distintas contribuciones al mismo: ferromagnética 1 (línea roja), ferromagnéticas 2 (línea verde), Langevin (línea celeste) y lineal (línea azul). 


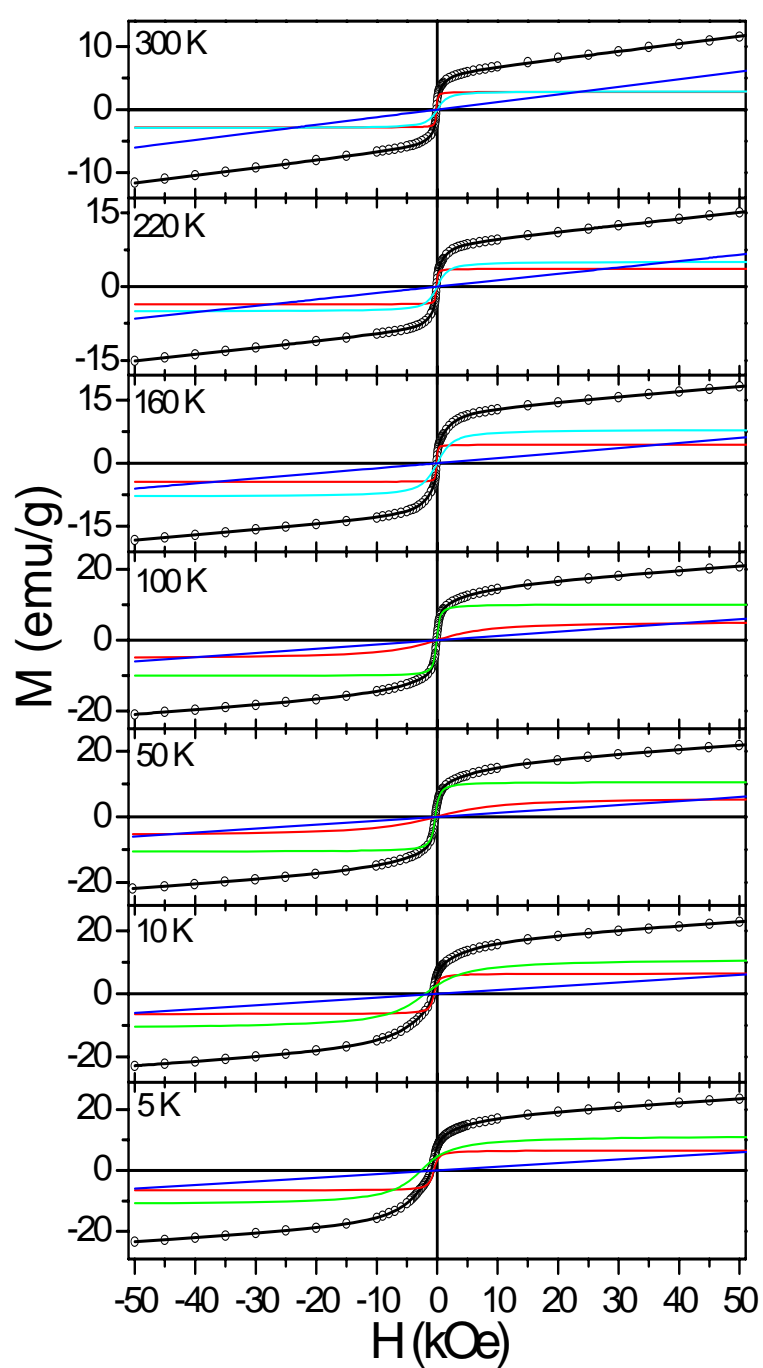

Figura 5.60: Resultados de los ajustes de los ciclos de histéresis medidos a bajas temperaturas para la muestra con $\mathrm{x}=0.25$. Ajuste propuesto (línea negra) y las distintas contribuciones al mismo: ferromagnética 1 (línea roja), ferromagnéticas 2 (línea verde), Langevin (línea celeste) y lineal (línea azul).

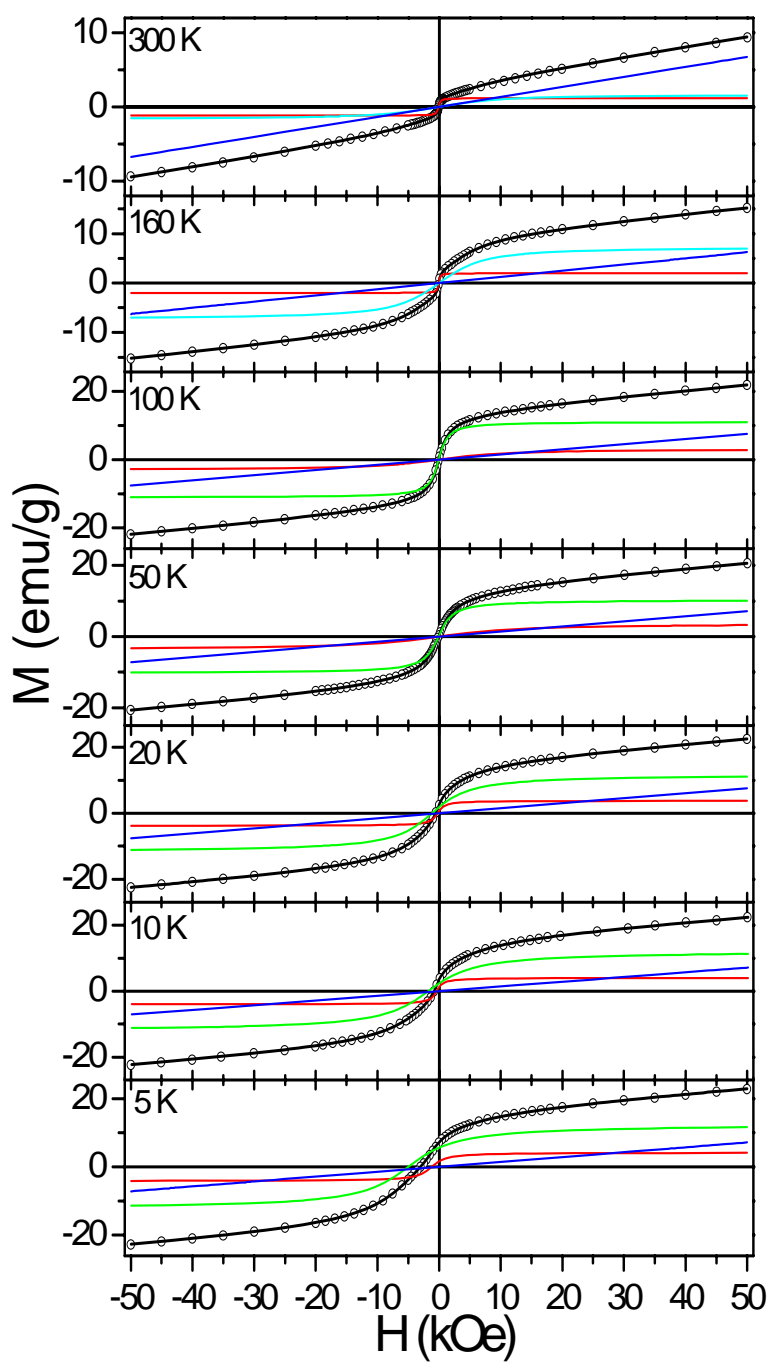

Figura 5.61: Resultados de los ajustes de los ciclos de histéresis medidos a bajas temperaturas para la muestra con $\mathrm{x}=0.30$. Ajuste propuesto (línea negra) y las distintas contribuciones al mismo: ferromagnética 1 (línea roja), ferromagnéticas 2 (línea verde), Langevin (línea celeste) y lineal (línea azul).

Los valores de los parámetros obtenidos de los ajustes de los ciclos de histéresis medidos a las diversas temperaturas se presentan a continuación en la Tabla 5.12. $\mathrm{M}_{\mathrm{S} 1}$ y $\mathrm{H}_{\mathrm{C} 1}$ son la magnetización de saturación y el campo coercitivo de la contribución ferromagnética de alta temperatura, $\mathrm{M}_{\mathrm{S} 2} \mathrm{y}_{\mathrm{C} 2}$ son los correspondientes a la contribución ferromagnética de baja temperatura, $\mathrm{M}_{\mathrm{SL}}$ y $\mu$ son la magnetización de saturación y el momento magnético de la componente de Langevin y $\chi_{\mathrm{A}}$ es la susceptibilidad de la componente lineal. 


\begin{tabular}{|c|c|c|c|c|c|c|c|c|}
\hline $\mathrm{x}$ & $\mathrm{T}$ & $\mathrm{M}_{\mathrm{s} 1}(\mathrm{emu} / \mathrm{g})$ & $\mathrm{H}_{\mathrm{cl} 1}(\mathrm{Oe})$ & $\mathrm{M}_{\mathrm{s} 2}(\mathrm{emu} / \mathrm{g})$ & $\mathrm{H}_{\mathrm{c} 2}(\mathrm{Oe})$ & $\mathrm{M}_{\mathrm{SL}}(\mathrm{emu} / \mathrm{g})$ & $\mu\left(\mu_{\mathrm{B}}\right)$ & $\chi_{\mathrm{A}}(\mathrm{emu} / \mathrm{gOe})$ \\
\hline \multirow{6}{*}{0.00} & 300 & $19.6_{2}$ & $220_{10}$ & - & - & $6.50_{2}$ & $2405_{20}$ & $1.9_{1} \times 10^{-5}$ \\
\hline & 160 & $21.0_{1}$ & $246_{12}$ & $5.5_{1}$ & $282_{14}$ & - & - & $2.3 \times 10^{-5}$ \\
\hline & 100 & $21.2_{1}$ & $312_{18}$ & $6.43_{8}$ & $376_{20}$ & - & - & $2.0_{2} \times 10^{-5}$ \\
\hline & 50 & $21.5_{1}$ & $411_{22}$ & $7.0_{1}$ & $563_{22}$ & - & - & $1.8_{1} \times 10^{-5}$ \\
\hline & 10 & $21.7_{1}$ & $530_{31}$ & $7.2_{1}$ & $711_{24}$ & - & - & $1.9_{1} \times 10^{-5}$ \\
\hline & 5 & $21.7_{1}$ & $577_{32}$ & $7.25_{4}$ & $805_{30}$ & - & - & $2.0_{1} \times 10^{-5}$ \\
\hline \multirow{7}{*}{0.10} & 300 & $11.3_{1}$ & $181_{11}$ & - & - & $5.68_{2}$ & $2543_{18}$ & $5.5_{1} \times 10^{-5}$ \\
\hline & 160 & $12.5_{1}$ & $212_{15}$ & $6.9_{1}$ & $265_{19}$ & - & - & $5.4_{1} \times 10^{-5}$ \\
\hline & 100 & $13.4_{1}$ & $284_{19}$ & $7.7_{1}$ & $483_{22}$ & - & - & $5.5_{1} \times 10^{-5}$ \\
\hline & 50 & $13.7_{1}$ & $382_{24}$ & $8.1_{1}$ & $647_{29}$ & - & - & $5.0_{2} \times 10^{-5}$ \\
\hline & 20 & $13.8_{1}$ & $454_{26}$ & $8.5_{1}$ & $872_{35}$ & - & - & $5.1_{1} \times 10^{-5}$ \\
\hline & 10 & $14.1_{1}$ & $471_{27}$ & $8.6_{1}$ & $985_{45}$ & - & - & $5.4_{2} \times 10^{-5}$ \\
\hline & 5 & $14.1_{1}$ & $508_{29}$ & $8.6_{1}$ & $1121_{44}$ & - & - & $5.3_{2} \times 10^{-5}$ \\
\hline \multirow{7}{*}{0.15} & 300 & $7.5_{1}$ & $151_{14}$ & - & - & $4.98_{1}$ & $2874_{25}$ & $8.0_{2} \times 10^{-5}$ \\
\hline & 220 & $8.6_{1}$ & $145_{13}$ & - & - & $6.76_{2}$ & $2315_{32}$ & $7.9_{2} \times 10^{-5}$ \\
\hline & 160 & $9.9_{2}$ & $178_{18}$ & $7.6_{1}$ & $207_{20}$ & - & - & $8.1_{2} \times 10^{-5}$ \\
\hline & 100 & $10.7_{2}$ & $258_{23}$ & $8.5_{1}$ & $471_{24}$ & - & - & $7.8_{2} \times 10^{-5}$ \\
\hline & 50 & $11.0_{1}$ & $322_{25}$ & $9.0_{1}$ & $706_{32}$ & - & - & $8.1_{2} \times 10^{-5}$ \\
\hline & 10 & $11.1_{1}$ & $451_{28}$ & $9.3_{1}$ & $1173_{39}$ & - & - & $8.1_{1} \times 10^{-5}$ \\
\hline & 5 & $11.2_{1}$ & $477_{27}$ & $9.4_{1}$ & $1461_{43}$ & - & - & $8.22 \times 10^{-5}$ \\
\hline \multirow{8}{*}{0.20} & 300 & $5.3_{1}$ & $138_{16}$ & - & - & $4.12_{2}$ & $2597_{15}$ & $10.0_{2} \times 10^{-5}$ \\
\hline & 220 & $6.8_{1}$ & $118_{15}$ & - & - & $5.60_{3}$ & $2107_{24}$ & $10.5_{1} \times 10^{-5}$ \\
\hline & 160 & $7.2_{1}$ & $134_{14}$ & $8.4_{1}$ & $20_{4}$ & - & - & $10.0_{2} \times 10^{-5}$ \\
\hline & 100 & $7.8_{1}$ & $183_{18}$ & $9.2_{1}$ & $249_{21}$ & - & - & $9.83 \times 10^{-5}$ \\
\hline & 50 & $8.5_{1}$ & $256_{21}$ & $9.6_{1}$ & $592_{33}$ & - & - & $10.2_{2} \times 10^{-5}$ \\
\hline & 20 & $8.9_{1}$ & $381_{25}$ & $9.7_{2}$ & $1161_{39}$ & - & - & $10.2_{2} \times 10^{-5}$ \\
\hline & 10 & $8.9_{1}$ & $435_{28}$ & $9.9_{1}$ & $1447_{48}$ & - & - & $10.0_{2} \times 10^{-5}$ \\
\hline & 5 & $9.0_{1}$ & $452_{28}$ & $10.0_{1}$ & $1790_{51}$ & - & - & $10.2_{2} \times 10^{-5}$ \\
\hline \multirow{7}{*}{0.25} & 300 & $2.80_{7}$ & $80_{8}$ & - & - & $2.88_{1}$ & $2816_{32}$ & $12.0_{3} \times 10^{-5}$ \\
\hline & 220 & $3.6_{1}$ & $82_{7}$ & - & - & $5.00_{2}$ & $2463_{43}$ & $12.3_{2} \times 10^{-5}$ \\
\hline & 160 & $4.4_{1}$ & $92_{8}$ & - & - & $7.60_{4}$ & $2323_{37}$ & $12.22 \times 10^{-5}$ \\
\hline & 100 & $5.3_{1}$ & $121_{11}$ & $10.1_{1}$ & $93_{7}$ & - & - & $12.5_{3} \times 10^{-5}$ \\
\hline & 50 & $5.9_{1}$ & $214_{20}$ & $10.6_{2}$ & $250_{19}$ & - & - & $12.1_{2} \times 10^{-5}$ \\
\hline & 10 & $6.4_{1}$ & $424_{25}$ & $11.1_{1}$ & $1649_{51}$ & - & - & $12.2_{2} \times 10^{-5}$ \\
\hline & 5 & $6.6_{1}$ & $431_{24}$ & $11.4_{1}$ & $2401_{58}$ & - & - & $12.1_{2} \times 10^{-5}$ \\
\hline \multirow{7}{*}{0.30} & 300 & $1.02_{5}$ & $40_{6}$ & - & - & $1.33_{1}$ & $2453_{26}$ & $15.1_{3} \times 10^{-5}$ \\
\hline & 160 & $2.4_{1}$ & $33_{5}$ & - & - & $7.00_{3}$ & $1966_{24}$ & $14.72 \times 10^{-5}$ \\
\hline & 100 & $3.1_{1}$ & $41_{6}$ & $11.1_{1}$ & $43_{5}$ & - & - & $14.8_{3} \times 10^{-5}$ \\
\hline & 50 & $3.7_{1}$ & $94_{9}$ & $11.6_{1}$ & $146_{12}$ & - & - & $15.1_{3} \times 10^{-5}$ \\
\hline & 20 & $3.81_{8}$ & $295_{21}$ & $11.9_{1}$ & $793_{36}$ & - & - & $14.8_{3} \times 10^{-5}$ \\
\hline & 10 & $4.00_{6}$ & $373_{22}$ & $12.1_{1}$ & $1983_{57}$ & - & - & $15.2_{3} \times 10^{-5}$ \\
\hline & 5 & $4.12_{7}$ & $428_{25}$ & $12.3_{1}$ & $2985_{61}$ & - & - & $15.1_{3} \times 10^{-5}$ \\
\hline
\end{tabular}

Tabla 5.12: Resultados de los ajustes de los ciclos de histéresis medidos entre $300 \mathrm{~K}$ y $5 \mathrm{~K}$. 
Primero se analizarán los resultados obtenidos para las medidas realizadas a temperatura ambiente, para las diferentes concentraciones. El comportamiento de la interacción ferromagnética de alta temperatura está caracterizado por los valores de la magnetización de saturación $\mathrm{M}_{\mathrm{s} 1} \mathrm{y}$ su campo coercitivo $\mathrm{H}_{\mathrm{cl} 1}$ ii En la figura 5.62 se puede observar que los valores de $\mathrm{M}_{\mathrm{s} 1}$ disminuyen con el contenido de $\mathrm{Cu}$ desde una valor de $20 \mathrm{emu} / \mathrm{g}$ para la muestra con $\mathrm{x}=0.00$ hasta un valor de 1 $\mathrm{emu} / \mathrm{g}$ para la muestras con $\mathrm{x}=0.30$.

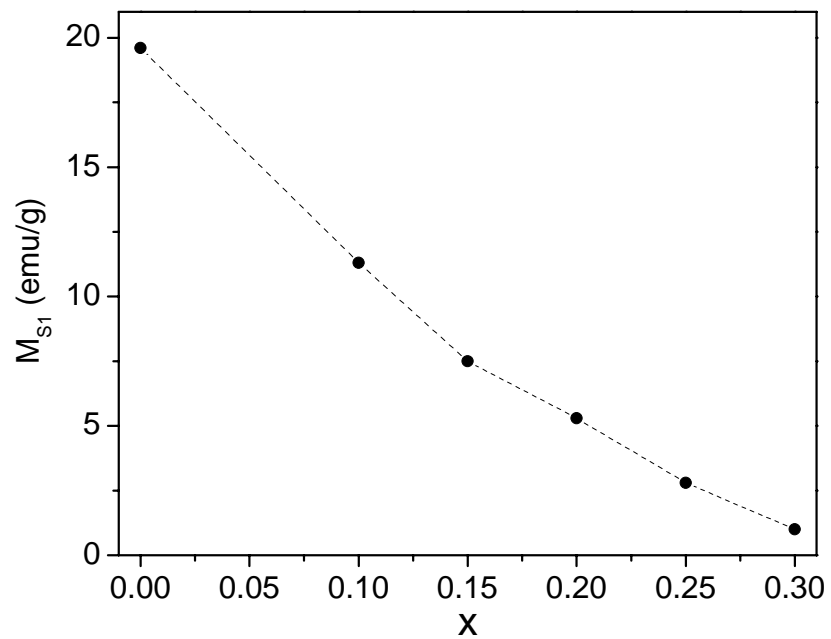

Figura 5.62: Evolución de la magnetización de saturación para las diferentes concentraciones estudiadas a temperatura ambiente.

Tanto por DRX como por EM se comprobó la presencia de la fase bcc-Fe(Mn,Cu), la cual es ferromagnética a temperatura ambiente, por esta razón es lógico asociar la contribución ferromagnética con esta fase. Como se discutió en la sección de resultados Mössbauer, esta fase magnética posee una contribución de un $18 \%$ para la muestra con $\mathrm{x}=0.00 \mathrm{y}$, debido al campo magnético hiperfino medio obtenido, se la pudo asociar con la presencia de $\mathrm{Fe}_{90} \mathrm{Mn}_{10}$. En base a esta información, y para determinar si efectivamente es plausible asociar la fase $\alpha-\mathrm{Fe}(\mathrm{Mn}, \mathrm{Cu})$ con la componente ferromagnética de los ciclos, se preparó una muestra de $\mathrm{Fe}_{90} \mathrm{Mn}_{10}$ a la cual se le realizó un ciclo de histéresis en las mismas condiciones que a las muestras estudiadas. La figura 5.63 muestra el resultado de la curva M-H para dicha muestra, junto con el ajuste propuesto para ella. Para la reproducción del ciclo de la muestra $\mathrm{Fe}_{90} \mathrm{Mn}_{10}$ se empleó una contribución ferromagnética, dada por la Ec. 4.7. El resultado del ajuste muestra una magnetización de saturación $\left(\mathrm{M}_{\mathrm{S}}^{*}\right)$ de $118_{1} \mathrm{emu} / \mathrm{g}$ y un campo coercitivo $\left(\mathrm{H}_{\mathrm{C}}^{*}\right)$ de $192{ }_{5}$ Oe.

\footnotetext{
ii El subíndice 1 en estos parámetros es colocado para diferenciarlos de sus pares cuando a bajas temperaturas para la reproducción de los ciclos haya que introducir una segunda interacción ferromagnética.
} 


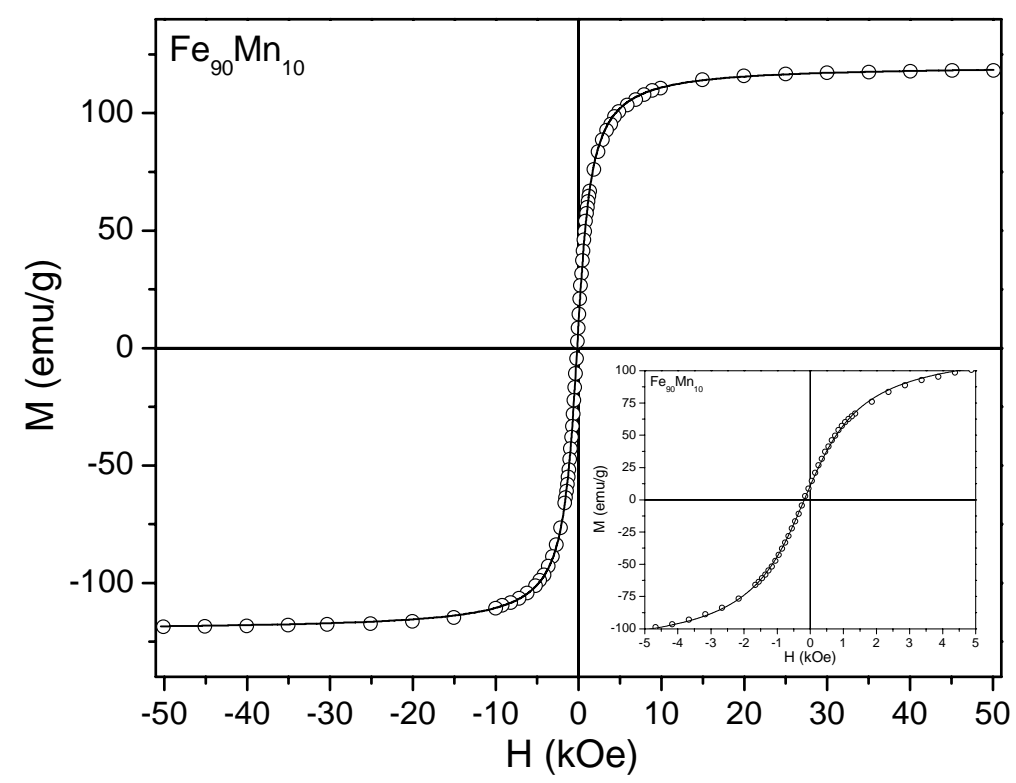

Figura 5.63: Ciclo de histéresis correspondiente a la muestra $\mathrm{Fe}_{90} \mathrm{Mn}_{10}$ medido a temperatura ambiente, junto con el ajuste propuesto (para mayor claridad se muestra sólo una rama del ciclo). Recuadro: ampliación de la región central del ciclo.

Empleando este resultado se pudo estimar que la magnetización de saturación $\mathrm{M}_{\mathrm{S} 1}$ obtenida en la muestra con $\mathrm{x}=0.00$ es del orden de 0.17 veces la magnetización de saturación obtenida para la muestra $\mathrm{Fe}_{90} \mathrm{Mn}_{10}$. Esto indica que un $17 \%$ de la muestra $\mathrm{Fe}_{79} \mathrm{Mn}_{21}$ está compuesta por $\mathrm{Fe}_{90} \mathrm{Mn}_{10}$, fracción que coincide con la obtenida para la fase $\alpha-\mathrm{Fe}(\mathrm{Mn})$ por EM. Por lo tanto, es posible asignar a la fase de estructura $b c c$ observada por DRX y por EM la contribución ferromagnética necesaria en la reproducción de los ciclos. Se debe aclarar que, decir que un $17 \%$ de la muestra está compuesta por $\mathrm{Fe}_{90} \mathrm{Mn}_{10}$ no es estrictamente la situación real ya que, existirá un gradiente composicional en la relación $\mathrm{Fe} / \mathrm{Mn}$, dando como resultado en EM un campo hiperfino promedio de $31 \mathrm{~T}$, el cual se asocia con la composición media $\mathrm{Fe}_{90} \mathrm{Mn}_{10}$, ocurriendo lo mismo, claro está, con la contribución a la magnetización de saturación de la componente ferromagnética en los ciclos de histéresis.

Este mismo análisis puede hacerse con el valor obtenido para $\mathrm{M}_{\mathrm{s} 1}$ para las muestras con $\mathrm{Cu}$, la única diferencia es que en estos casos sólo en las muestras con $\mathrm{x} \leq 0.15$ es posible observar por EM la presencia de la fase magnética. Además, en estos casos, no es posible determinar si el campo hiperfino medio corresponde a la fase $\alpha-\mathrm{Fe}(\mathrm{Mn})$ o a fase $\alpha-\mathrm{Fe}(\mathrm{MnCu})$. Debido a esto se escogió, como primera aproximación, suponer que la fase responsable del comportamiento de $\mathrm{M}_{\mathrm{S1}}$ es la $\alpha$ $\mathrm{Fe}(\mathrm{Mn})$, aún en las muestras con $\mathrm{Cu}$. Aceptando esta hipótesis, se puede realizar un análisis similar para la magnetización de saturación de la componente ferromagnética de alta temperatura en las muestras con $\mathrm{Cu}$. De esta manera, comparando ahora la magnetización de saturación obtenida para 
las muestras con distintas concentraciones de $\mathrm{Cu}$ con la obtenida para la muestra $\mathrm{Fe}_{90} \mathrm{Mn}_{10}$, se puede determinar qué fracción de esta última está presente en cada aleación. A continuación, la Tabla 5.13 muestra el resultado del cociente entre la magnetización de saturación de la muestra $\left(\mathrm{Fe}_{79} \mathrm{Mn}_{21}\right)_{1-\mathrm{x}} \mathrm{Cu}_{\mathrm{x}}\left(\mathrm{M}_{\mathrm{s} 1}\right)$ y la magnetización de saturación de la muestra $\mathrm{Fe}_{90} \mathrm{Mn}_{10}\left(\mathrm{M}_{\mathrm{S}}^{*}\right)$. Para hacer más fácil la comparación también se incluyen los valores de las fracciones obtenidas para la fase $\alpha$ $\mathrm{Fe}(\mathrm{MnCu})$ tomadas de la Tabla 5.7.

\begin{tabular}{|c|c|c|c|c|c|c|}
\hline & $\mathrm{x}=0.00$ & $\mathrm{x}=0.10$ & $\mathrm{x}=0.15$ & $\mathrm{x}=0.20$ & $\mathrm{x}=0.25$ & $\mathrm{x}=0.30$ \\
\hline $\mathrm{M}_{\mathrm{s} 1} / \mathrm{M}_{\mathrm{S}}^{*}$ & $0.17_{1}$ & $0.10_{1}$ & $0.06_{1}$ & $0.04_{1}$ & $0.02_{1}$ & $0.01_{1}$ \\
\hline $\mathrm{f}$ & 0.18 & 0.10 & 0.05 & - & - & - \\
\hline
\end{tabular}

Tabla 5.13: Cociente entre la magnetización de saturación $\mathrm{M}_{\mathrm{S} 1}$ y la correspondiente a la muestra $\mathrm{Fe}_{90} \mathrm{Mn}_{10}$ junto con la fracción (f) de la fase $\alpha-\mathrm{Fe}(\mathrm{MnCu})$ obtenida por EM.

Se observa que la fracción de $\mathrm{Fe}_{90} \mathrm{Mn}_{10}$, que debería estar presente en las muestras con $\mathrm{x} \leq$ 0.15 según los ciclos de histéresis está en muy buen acuerdo con las fracciones obtenidas para la fase $\alpha-\mathrm{Fe}(\mathrm{MnCu})$ por EM. Por otra parte, las fracciones para las muestras con $\mathrm{x} \geq 0.20$ están en el límite de resolución de la técnica de EM, lo que, sumado al gran efecto en la parte central del espectro, hacen que la detección de esta fase por EM sea casi imposible en estas concentraciones. El magnetómetro SQUID, es mucho más sensible a la respuesta magnética del sistema y entonces es posible encontrar una señal que provenga de un material ferromagnético presente en 1 ppm y por lo tanto, fracciones como las encontradas en las muestras con $\mathrm{x} \geq 0.20$ son detectables.

Las figuras 5.64 y 5.65 muestran los resultados obtenidos de los ajustes de los ciclos de histéresis para la magnetización de saturación de las contribuciones ferromagnética 1 y 2 , respectivamente. Se observa que la magnetización de saturación de la componente ferromagnética $1\left(\mathrm{Ms}_{1}\right)$ presenta una leve disminución con la temperatura en todas las concentraciones. El valor de $\mathrm{Ms}_{1}$, es mayor para la muestra $\sin \mathrm{Cu}$ y disminuye a medida que el contenido de $\mathrm{Cu}$ se incrementa. Esta componente está asociada con la fase $\alpha-\mathrm{Fe}(\mathrm{MnCu})$ magnéticamente ordenada a temperatura ambiente, por lo tanto, el hecho de que el valor de la magnetización de saturación aumente débilmente para todas las muestras al disminuir la temperatura es esperable.

En lo que respecta a $\mathrm{Ms}_{2}$ se nota que su valor también aumenta al disminuir la temperatura, siendo el aumento más pronunciado que en $\mathrm{Ms}_{1}$. Una de las características más interesantes en el comportamiento de $\mathrm{Ms}_{2}$ es que el valor más alto lo presenta la muestra con $\mathrm{x}=0.30 \mathrm{y}$ disminuye para las concentraciones menores. Esta tendencia, la cual es opuesta a la presentada por $\mathrm{Ms}_{1}$, es similar a la encontrada a bajas temperaturas por EM en el campo magnético hiperfino de la 
distribución de bajo campo. Esto es un claro indicio de que esta nueva fase, que aparece a bajas temperaturas, tiene una correlación con la línea central de los espectros Mössbauer. Probablemente esta nueva fase magnética esté también ligada con la presencia de partículas interactuantes con un comportamiento de tipo vidrio de espín pues comienza a ser más significativa a medida que el contenido de $\mathrm{Cu}$ aumenta, de modo similar a como se manifiesta en las medidas de susceptibilidad $\mathrm{AC}$.

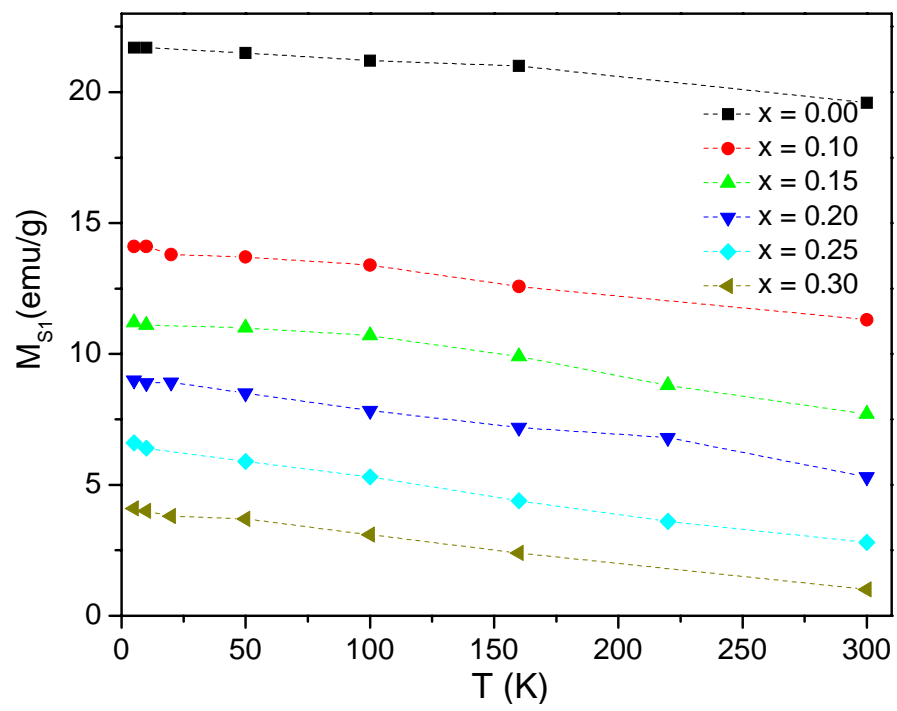

Figura 5.64: Magnetización de saturación de la componente ferromagnética 1 obtenida de los ajustes de los ciclos entre $300 \mathrm{~K}$ y $5 \mathrm{~K}$.

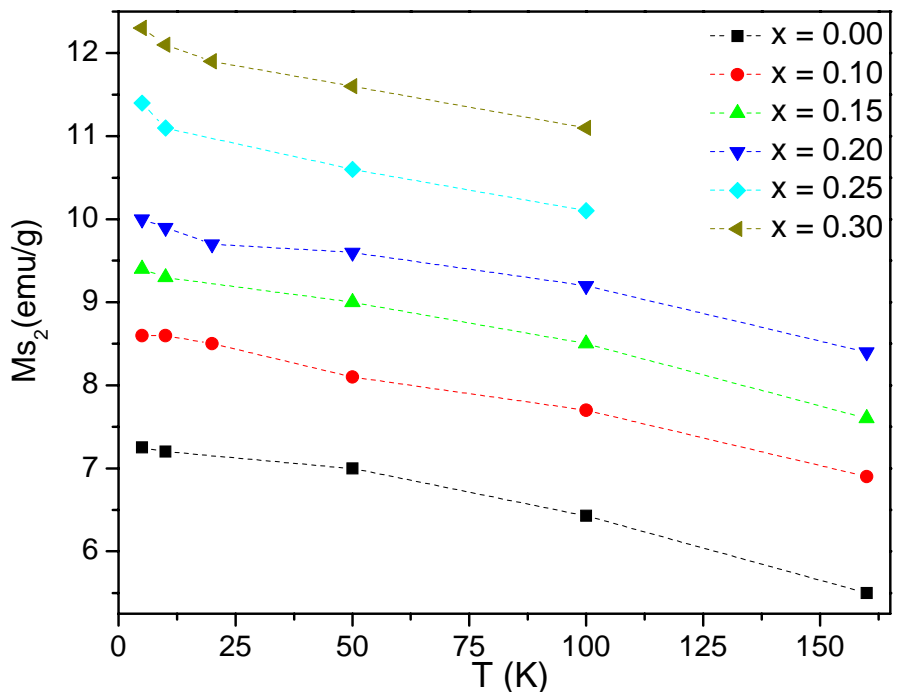

Figura 5.65: Magnetización de saturación de la componente ferromagnética 2 obtenida de los ajustes de los ciclos entre $300 \mathrm{~K}$ y $5 \mathrm{~K}$. 
Las figuras 5.66 y 5.67 muestran el campo coercitivo de las contribuciones ferromagnéticas 1 y 2 obtenidos de los ajustes de los ciclos de histéresis. Para todas las muestras $\mathrm{Hc}_{1}$ y $\mathrm{Hc}_{2} \mathrm{se}$ incrementa a medida que la temperatura disminuye. Este comportamiento puede ser pensado como un aumento de la dificultad para que la magnetización del sistema pueda acompañar la inversión del campo externo aplicado. En cuanto al valor de $\mathrm{Hc}_{1}$, es mayor para la muestra $\sin \mathrm{Cu}, \mathrm{y}$ disminuye paulatinamente al incorporar este último a la aleación, para toda la región de temperaturas. Si bien, como una primera aproximación, se supuso que la componente ferromagnética 1 está asociada con la presencia de regiones de composición media $\mathrm{Fe}_{90} \mathrm{Mn}_{10}$, el comportamiento de $\mathrm{Hc}_{1}$ con la concentración muestra que parte del $\mathrm{Cu}$ se incorporó también a la fase bcc. La evolución de $\mathrm{Hc}_{2}$ es más compleja que la presentada por $\mathrm{Hc}_{1}$. El aumento del valor de $\mathrm{Hc}_{2}$ al disminuir la temperatura es más pronunciado que el de $\mathrm{Hc}_{1}$, sobre todo en las muestras con los contenidos de $\mathrm{Cu}$ más altos, donde se alcanzan valores de hasta $0.3 \mathrm{~T}$. A las temperaturas más altas las muestras con menor contenido de $\mathrm{Cu}$ son las que presentan un valor de $\mathrm{Hc}_{2}$ más alto, invirtiéndose totalmente este comportamiento a las temperaturas más bajas. Nuevamente, este comportamiento de inversión recuerda la evolución del campo magnético hiperfino (contribución de bajo campo) obtenido por EM (figura 5.39). Este hecho refuerza, junto con el comportamiento de $\mathrm{Ms}_{2}$, que esta fase de baja temperatura tiene su correlato con la fase $f c c-\mathrm{FeMnCu}$ observada a temperatura ambiente. 


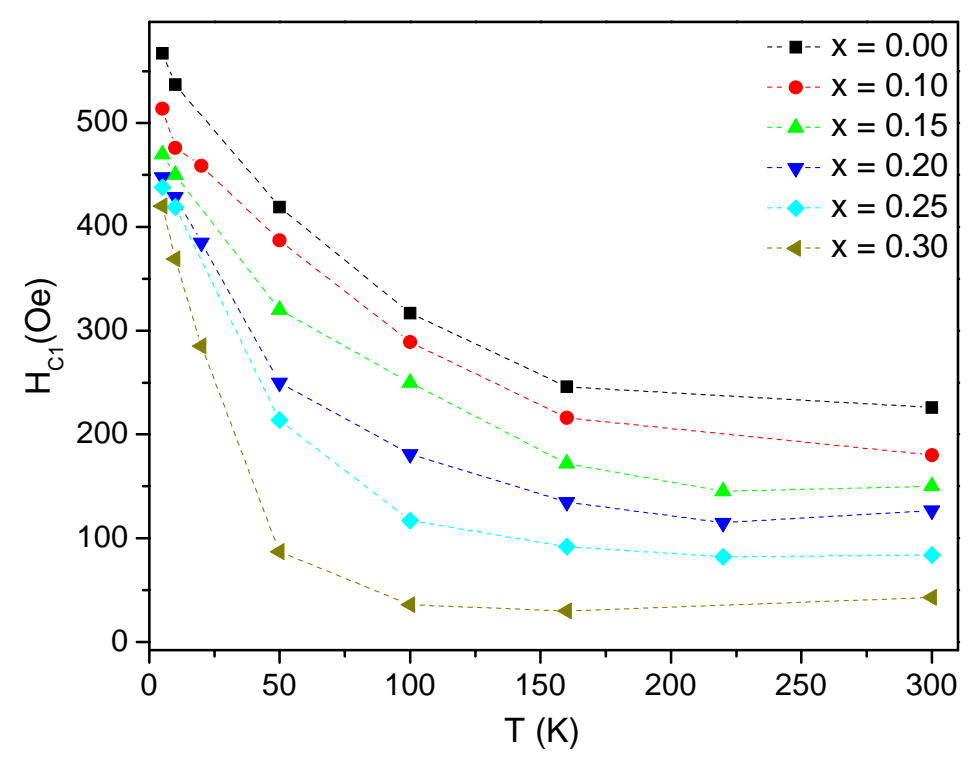

Figura 5.66: Campo coercitivo de la componente ferromagnética 1 obtenida de los ajustes de los ciclos entre $300 \mathrm{~K}$ y $5 \mathrm{~K}$.

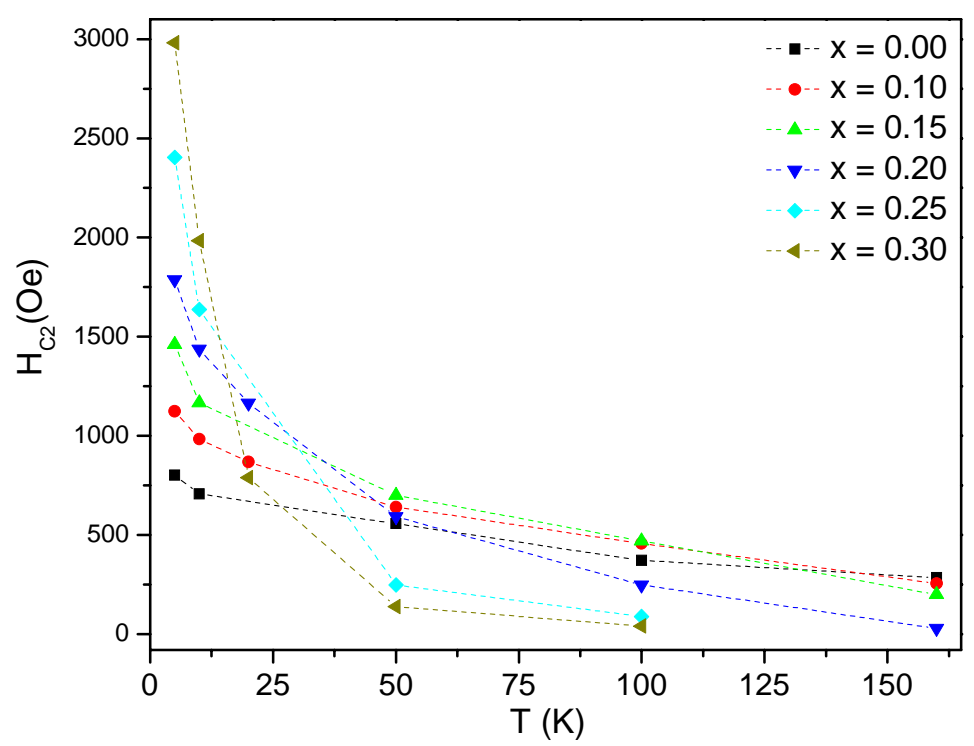

Figura 5.67: Campo coercitivo de la componente ferromagnética 2 obtenida de los ajustes de los ciclos entre $300 \mathrm{~K}$ y $5 \mathrm{~K}$.

Por último, la figura 5.68 muestra los resultados obtenidos de los ajustes para la componente lineal $\left(\chi_{\mathrm{A}}\right)$. Se puede descartar que esta contribución provenga de partículas en estado paramagnético ya que de ser así se espera que el valor $\chi_{\mathrm{A}}$ disminuya a medida que la temperatura aumenta, acorde a la ley de Curie. ${ }^{49}$ También a bajas temperaturas la magnetización a altos campos 
debería comenzar a apartarse del comportamiento lineal, para adoptar la forma curvada que predice la función de Brillouin. ${ }^{50}$ Por lo tanto, esta contribución debe estar ligada al carácter antiferromagnético de las muestras. Sumado a esto, el comportamiento tipo vidrio de espín encontrado en la susceptibilidad AC también puede explicar el aumento en el valor de $\chi_{\mathrm{A}}$ con la concentración de $\mathrm{Cu}$. Se conoce que los vidrios de espín canónicos no alcanzan la saturación en los ciclos de histéresis, aún para campos magnéticos muy elevados debido a la competencia entre las interacciones presentes en los mismos. ${ }^{51}$

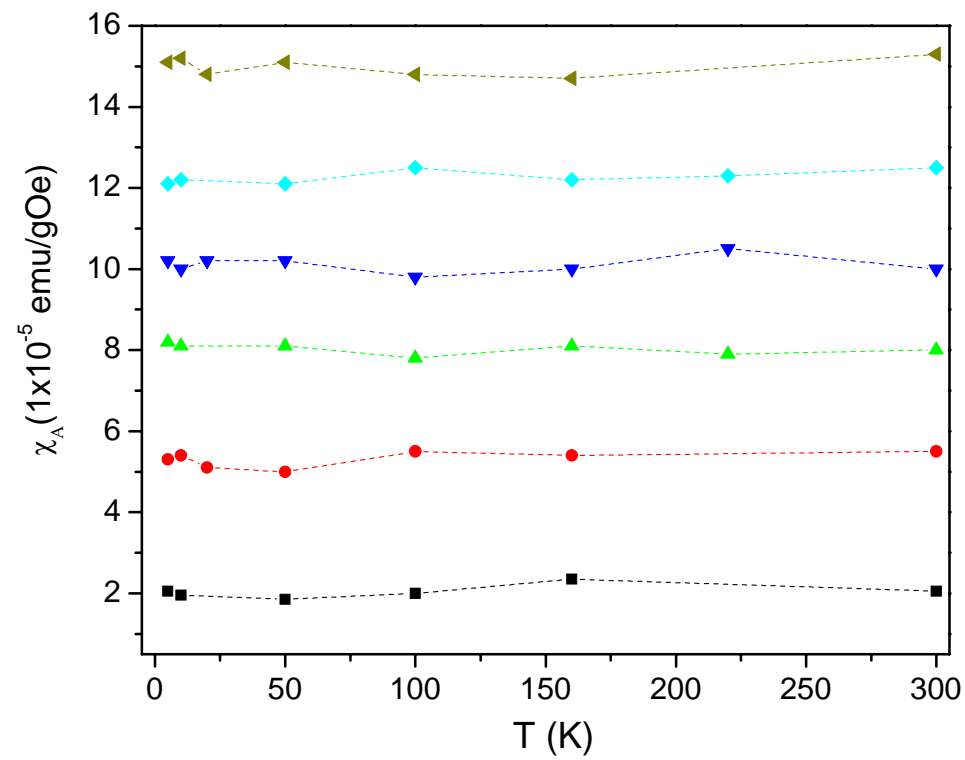

Figura 5.68: Resultados para la componente lineal obtenida de los ajustes de los ciclos entre $300 \mathrm{~K}$ y $5 \mathrm{~K}$.

Continuando con el estudio histerético, se realizaron en las muestras con $\mathrm{x}=0.00, \mathrm{x}=0.20 \mathrm{y}$ $\mathrm{x}=0.30$ medidas enfriando las muestras en presencia de un campo magnético. Este tipo de estudio es empleado para determinar la presencia del efecto denominada exchange bias, en el cual se observa un desplazamiento lateral de la curva de histéresis con respecto a la medida realizada enfriando sin campo. ${ }^{52}$ En las figuras 5.69 a 5.71 se presentan los resultados obtenidos para las diferentes muestras y temperaturas, empleando un campo de enfriado $\left(H_{F C}\right)$ de $20 \mathrm{kOe}$. Si bien los ciclos fueron medidos entre $-50 \mathrm{kOe}$ y $50 \mathrm{kOe}$ en las figuras se muestra la zona central para más claridad. Se observa un corrimiento de las curvas de histéresis hacia campos negativos, siendo este desplazamiento más evidente para las más bajas temperaturas y los mayores contenidos de $\mathrm{Cu}$. 


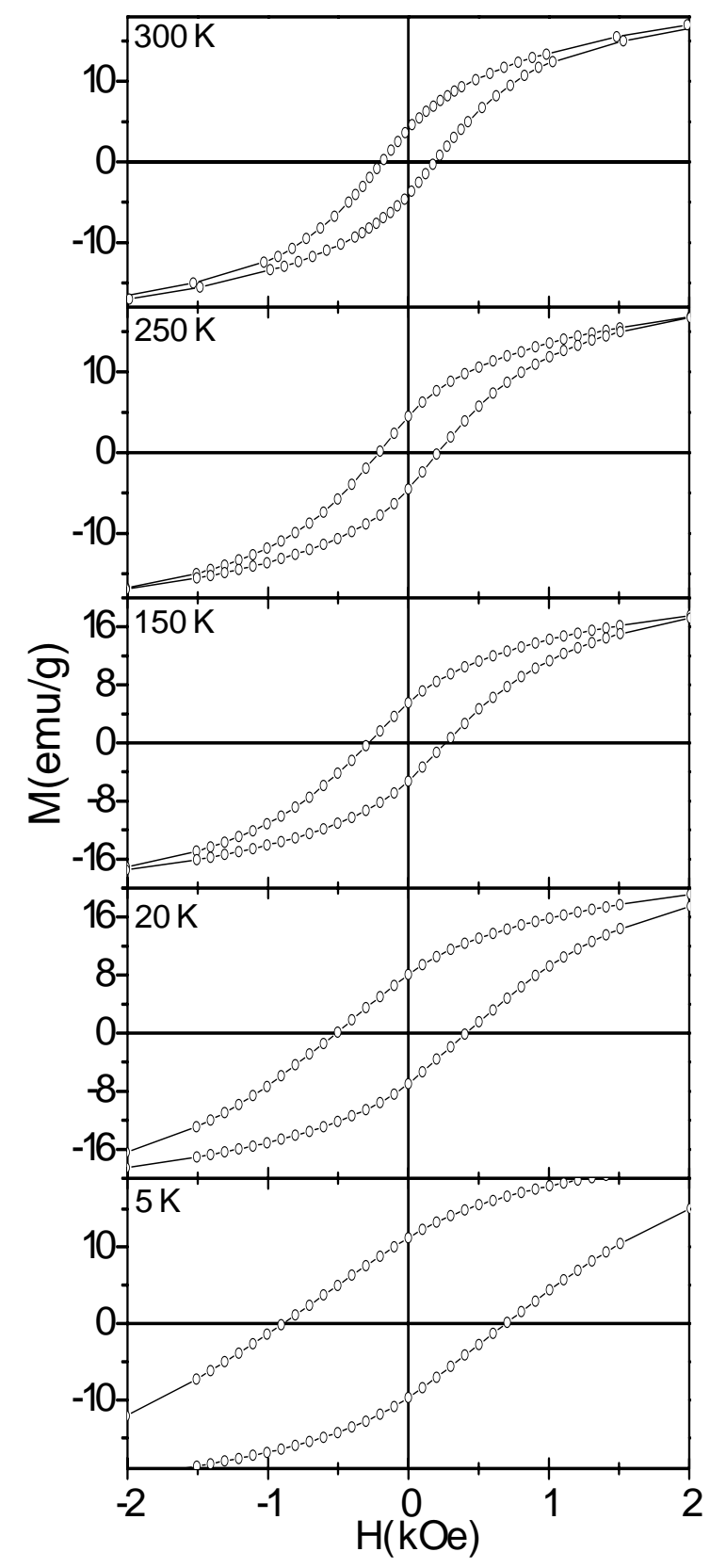

Figura 5.69: Ciclos de histéresis para la muestra $\mathrm{x}=$ 0.00 enfriada en un campo magnético de $20 \mathrm{kOe}$.

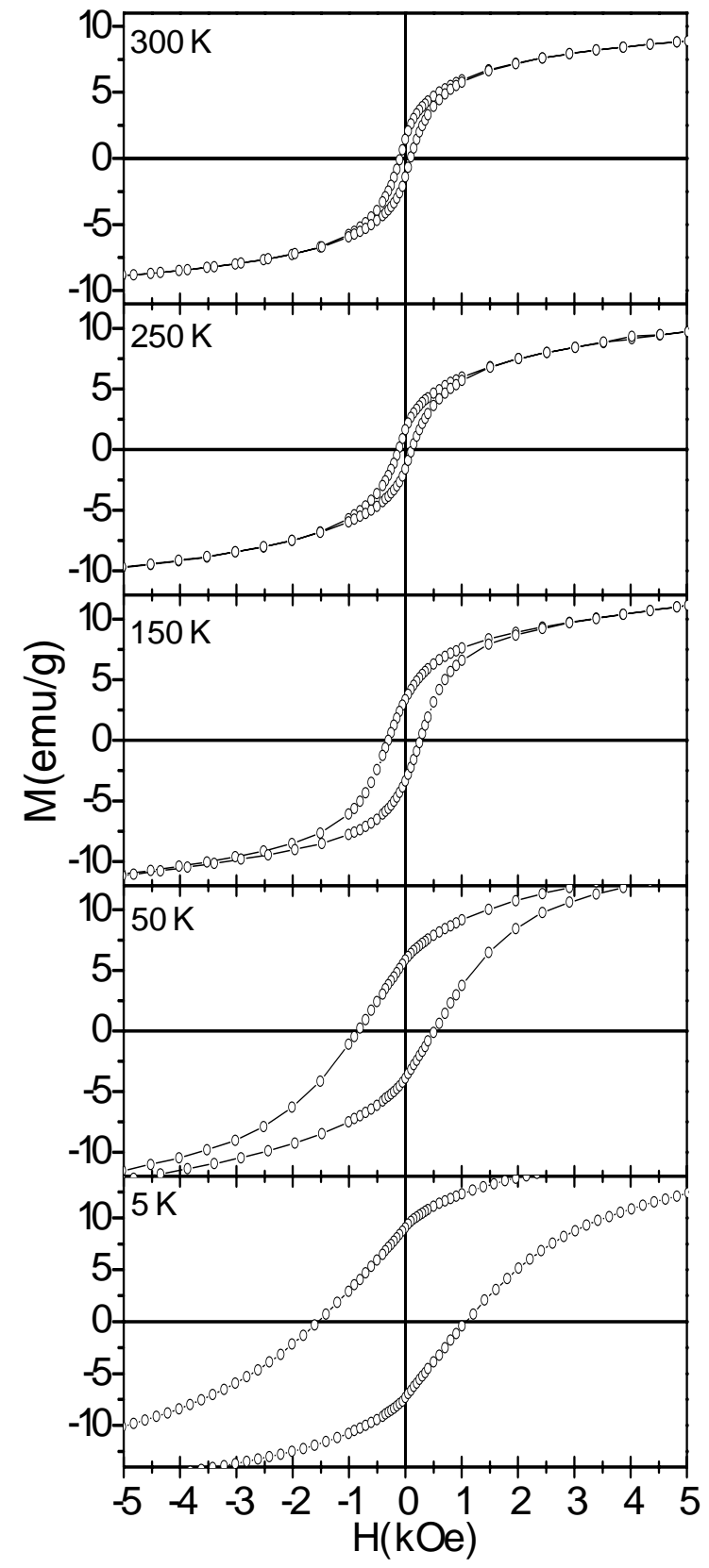

Figura 5.70: Ciclos de histéresis para la muestra $\mathrm{x}=$ 0.20 enfriada en un campo magnético de $20 \mathrm{kOe}$. 


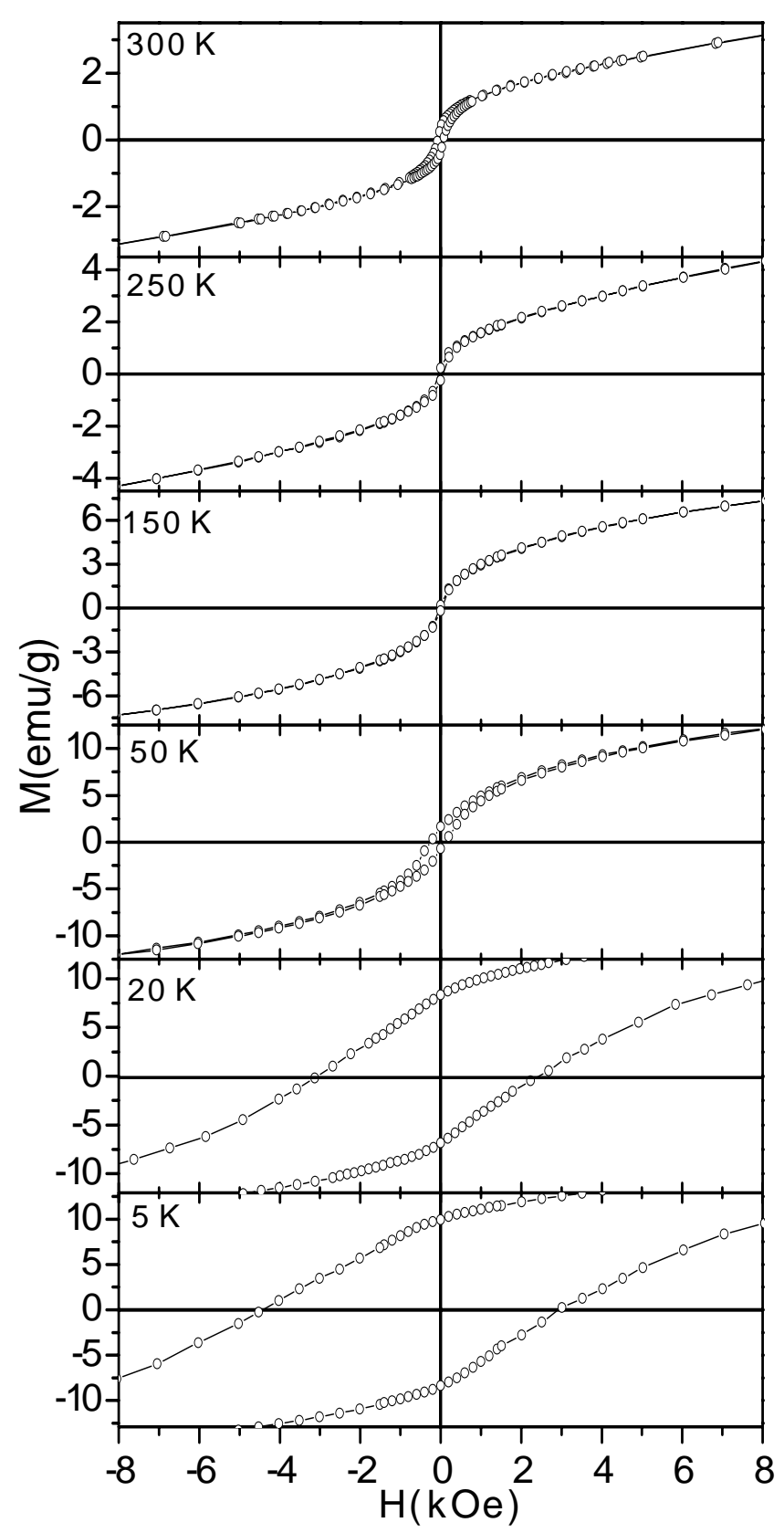

Figura 5.71: Ciclos de histéresis para la muestra $\mathrm{x}=0.30$ enfriada en un campo magnético de 20 kOe.

La presencia del campo magnético durante el enfriado produce un corrimiento de los ciclos hacia el lado izquierdo, efecto que se hace más notorio cuanto menor es la temperatura de medida. Para cuantificar el orden de este desplazamiento con la temperatura se usará la habitual definición para el campo de exchange bias $^{53}$ dada por: 


$$
\mathrm{H}_{\mathrm{EB}}=\frac{\left|\mathrm{H}_{\mathrm{CD}}\right|-\left|\mathrm{H}_{\mathrm{CI}}\right|}{2}
$$

donde $\mathrm{H}_{\mathrm{CD}}$ y $\mathrm{H}_{\mathrm{CI}}$ son los campos coercitivos del ciclo a derecha e izquierda, respectivamente. En la figura 5.72 se presenta el comportamiento del $H_{E B}$ en función de la temperatura para las tres muestras estudiadas.

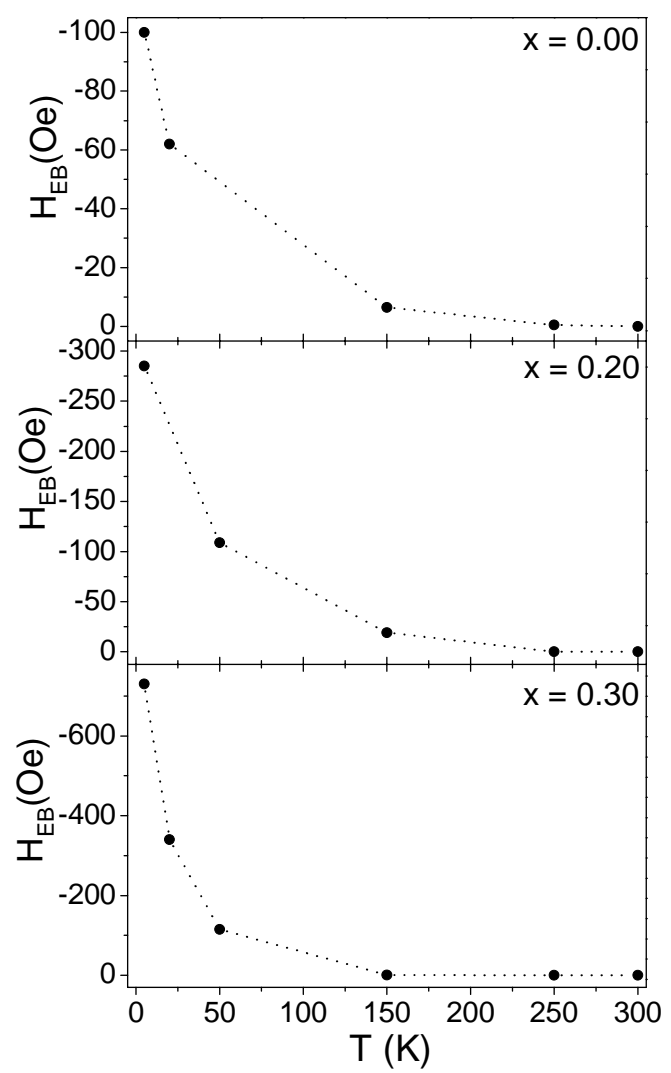

Figura 5.72: Comportamiento del campo de exchange bias (izq) y el campo coercitivo medio (der.) en función de la temperatura para las muestras $\mathrm{x}=0.00, \mathrm{x}=0.20 \mathrm{y} \mathrm{x}=0.30$.

Se observa en la figura que para todas las concentraciones $H_{E B}$ es nulo a $300 \mathrm{~K}$, debido a que, no se realizó un enfriado desde una temperatura superior. A medida que desciende la temperatura en presencia de campo, el valor de $H_{E B}$ aumenta, en valor absoluto. En estas condiciones, la primera muestra que presenta una variación es la $\mathrm{x}=0.00$, donde a $250 \mathrm{~K}$ el campo de exchange bias resulta distinto de cero. A $150 \mathrm{~K}$ la muestra con $\mathrm{x}=0.20$ muestra un corrimiento del ciclo $\mathrm{y}$, recién por debajo de esta temperatura se observan cambios en la muestra $\mathrm{x}=0.30$. La secuencia en la que se presentan los cambios en $H_{E B}$, es decir, comienzan a aparecer a temperaturas menores para los mayores contenidos de $\mathrm{Cu}$, es similar al comportamiento observado tanto en el campo magnético 
hiperfino como en la susceptibilidad $\mathrm{AC}$ en función de la temperatura, por lo que es plausible atribuir el corrimiento de los ciclos al carácter tipo vidrio de espín de las muestras.

La figura 5.73 muestra como el ciclo de histéresis de la aleación con $\mathrm{x}=0.30$ es afectado cuando la misma es enfriada desde temperatura ambiente hasta $5 \mathrm{~K}$ en presencia de distintos campos magnéticos. Se observa que a medida que el campo magnético de enfriado aumenta el efecto del corrimiento del ciclo es más notorio. Para poder apreciar mejor este comportamiento, la figura 5.74 muestra la variación del campo de exchange bias en función del campo magnético.
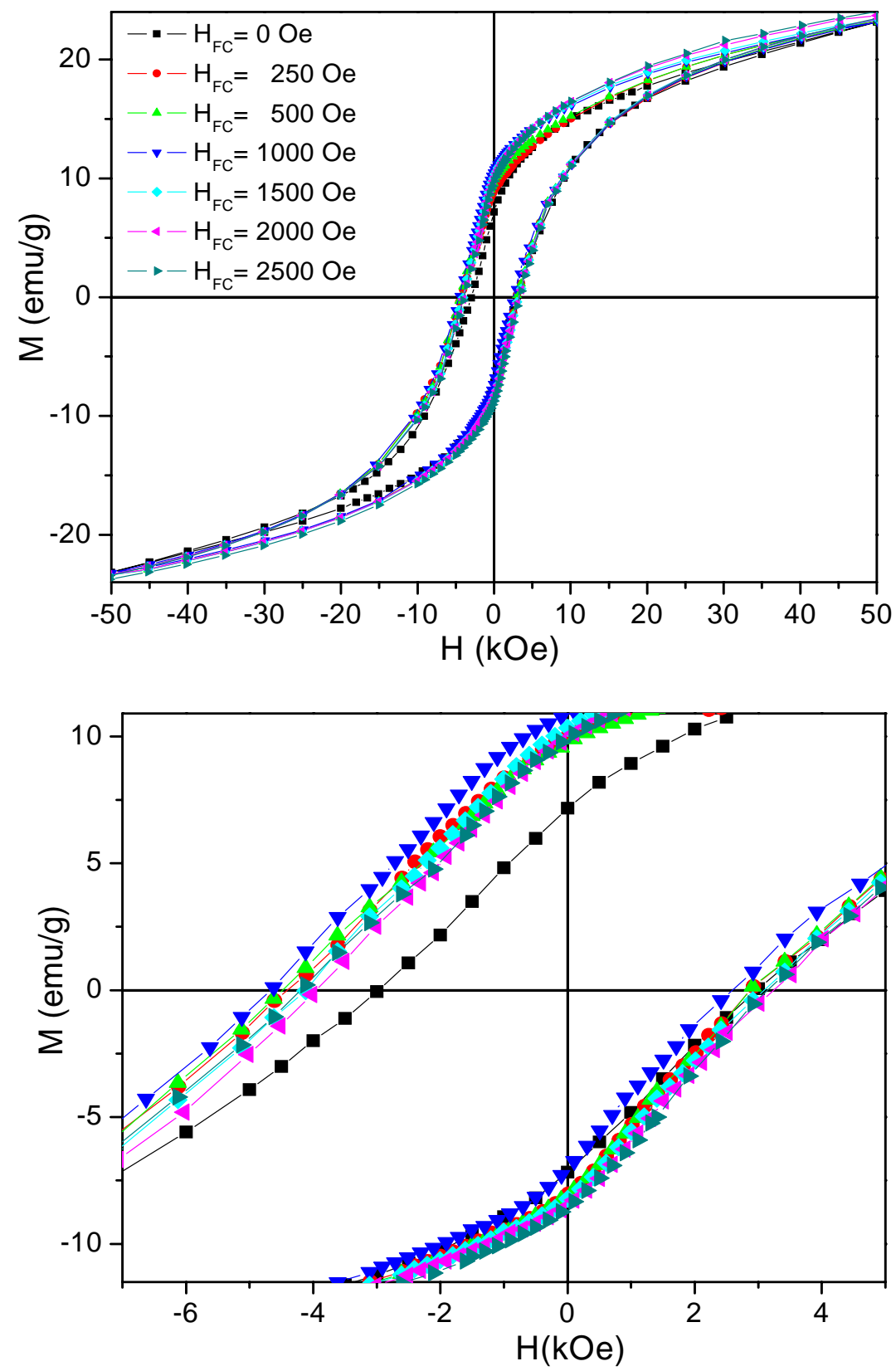

Figura 5.73: Arriba: Ciclo de histéresis para la muestra $\mathrm{x}=0.30$ medido a $5 \mathrm{~K}$ para distintos campos de enfriado $\left(H_{F C}\right)$. Abajo: ampliación de la región central del ciclo. 


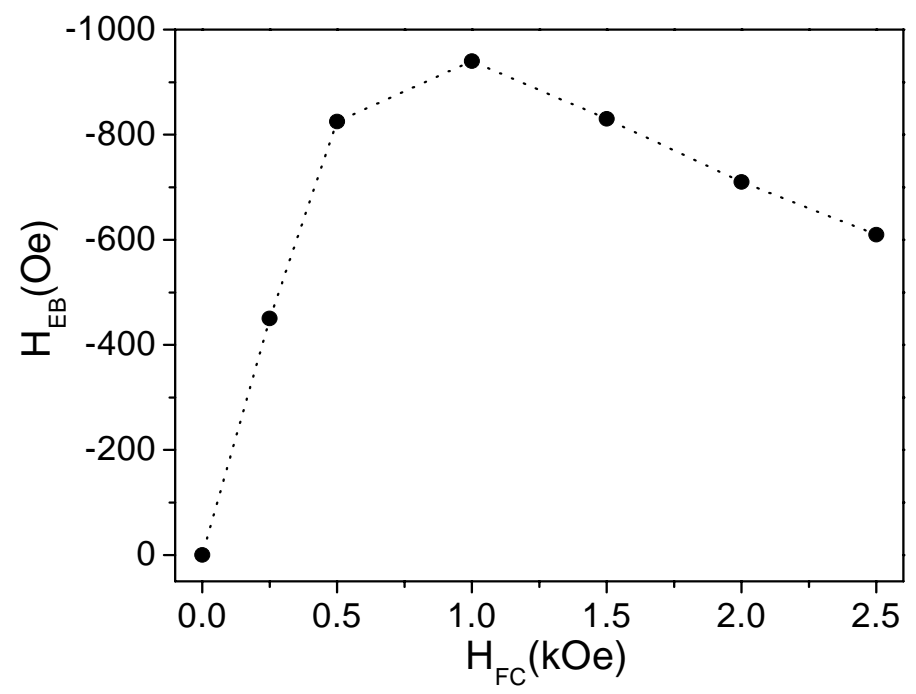

Figura 5.74: Campo de exchange bias en función del campo magnético de enfriado.

Otro resultado interesante es presentado en la figura 5.75, donde se aplica un campo de $H_{F C}=$ 20 kOe a partir de una determinada temperatura $\left(T_{F C}\right)$ hasta llegar a $5 \mathrm{~K}$, temperatura a la cual se realizó la medida. En este caso si se aplica el campo magnético a temperaturas muy por encima de $T_{\max }$ el efecto de exchange bias es más grande que cuando se lo realiza a temperaturas por debajo de este punto donde el efecto tiende a desaparecer.

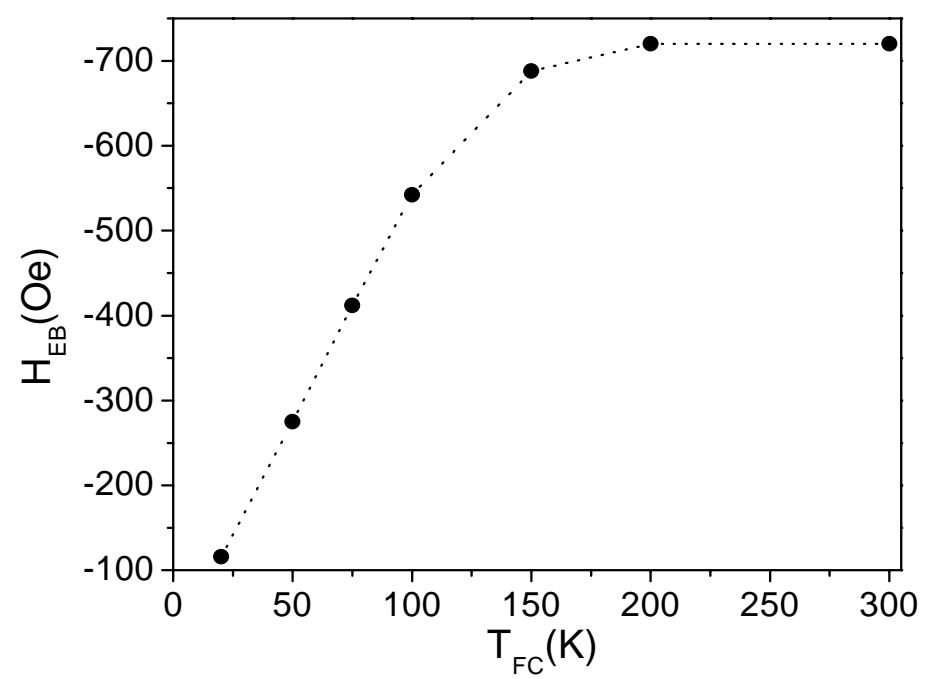

Figura 5.75: Campo de exchange bias medida a $5 \mathrm{~K}$ después de ser enfriado en un campo de $20 \mathrm{kOe}$ desde la temperatura $T_{F C \text {. }}$ 
La propiedad de exchange bias se encontró originalmente en el estudio de sistemas de partículas de Co rodeadas por una capa de óxido de cobalto. ${ }^{54}$ Desde ese entonces las investigaciones en torno a este fenómeno se basaron principalmente en el estudio de sistemas multicapas de materiales ferromagnéticos (FM) y antiferromagnéticos (AFM). Además de en estos sistemas el fenómeno de exchange bias ha sido observado en muestras que involucren una interfaz ferrimagnética $(\mathrm{FI}) /(\mathrm{AFM})$ o una $(\mathrm{FM}) /(\mathrm{FI}),{ }^{55} \mathrm{o}$ en sistemas que presentan una fase de vidrio de espín (SG) cuando forman interfases $\mathrm{FI} / \mathrm{SG}^{56} \mathrm{o} \mathrm{AFM} / \mathrm{SG}^{57}$

La presencia que este efecto en las muestras brinda un indicio importante para proponer un modelo del sistema estudiado. Como se mencionó en el párrafo anterior el efecto de exchange bias está asociado a la presencia de una interfase entre capas con diferentes órdenes magnéticos. Con esto en mente se puede proponer el siguiente modelo: las partículas están compuestas por un núcleo magnéticamente ordenado (AF o FI) y una superficie estructuralmente desordenada que presenta propiedades tipo vidrio de espín. En este caso se puede esperar una fuerte influencia del campo de enfriado en el fenómeno de exchange bias producto de las múltiples configuraciones equivalentes existentes en una fase tipo vidrio de espín, tal como es observado en la figura 5.75. Esto es debido a que de acuerdo al valor del campo de enfriado, aplicado por encima de la temperatura de congelamiento del sistema, $T_{\max }$, la magnetización de la fase ordenada tenderá a alinearse en mayor o menor medida con la dirección del campo externo. Cuando la temperatura desciende pasando por $T_{\max }$ el sistema desordenado se congelará en una configuración de las múltiples posibles por medio de la interacción de intercambio con la fase ordenada. De este modo, dependiendo del valor del campo de enfriado, la degeneración de la fase tipo vidrio de espín puede ser reducida, pudiendo si el campo magnético es lo suficientemente intenso destruir completamente dicha fase. Es importante ver la diferencia que existe entre este modelo y uno compuesto por una interfase FM/AFM. En un sistema uniaxial con estás características sólo existirán para la capa AFM dos posibles configuraciones de espín energéticamente equivalentes, por lo tanto cuando se continua con el proceso de enfriado la interacción de intercambio con la capa FM podrá optar sólo por uno de estos dos estados. 


\section{Medidas de magnetización con y sin campo (FC-ZFC)}

Por último se realizaron medidas de magnetización en función de la temperatura enfriando con $(F C)$ y $\sin (Z F C)$ campo magnético aplicado. A continuación en la figura 5.76 se muestran los resultados obtenidos para las curvas ZFC y FC para enfriando en campos magnéticos ${ }^{\mathrm{iii}}$ de 20 Oe y 100 Oe.
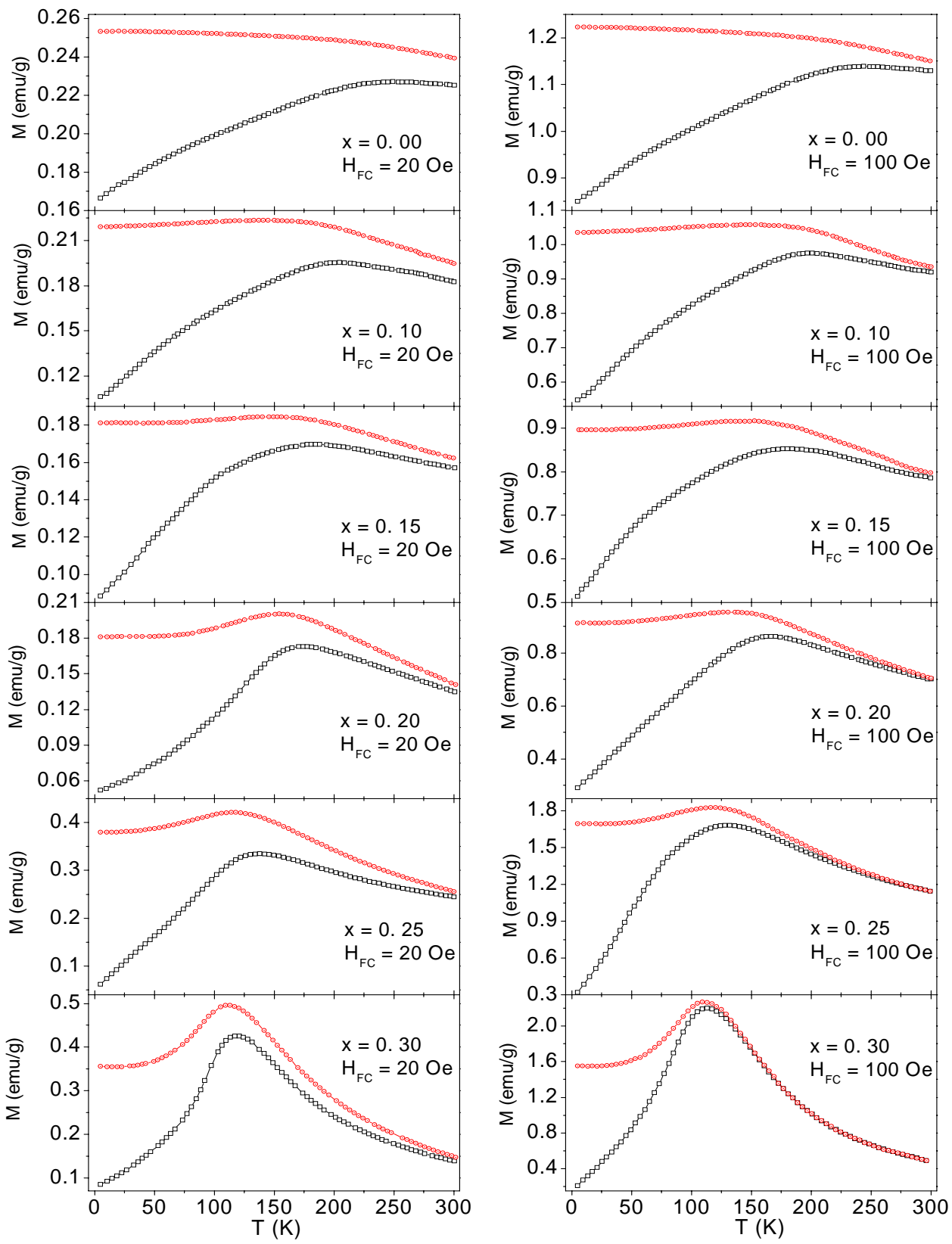

Figura 5.76: Magnetización en función de la temperatura en FC y ZFC para campos de enfriado de 20 Oe (izq.) y $100 \mathrm{Oe}$ (der.).

\footnotetext{
${ }^{\text {iii }}$ El valor del campo magnético de enfriado es el mismo que posteriormente se emplea para realizar la medida de la magnetización.
} 
Se observa de la figura anterior, la curva de magnetización $F C$ posee valores de magnetización superiores a los presentados por la curva ZFC para todas las concentraciones en la mayor parte del intervalo de temperaturas estudiado. Este hecho es consecuencia de que al enfriar al sistema en presencia de un campo magnético se impone una dirección preferencial a los momentos magnéticos de la muestra. La diferencia entre las curvas ZFC y FC muestra que existe una clara irreversibilidad en el proceso de medida. Se suele definir ${ }^{58}$ la temperatura de irreversibilidad $\left(T_{i}\right)$ como aquella en que la diferencia entre los valores de las curvas ZFC y FC es un $5 \%$ del valor del máximo de la curva ZFC (ver Tabla 5.14). En un vidrio de espín canónico esta temperatura se encuentra cercana a la temperatura del máximo de la curva $Z F C\left(T_{Z F C}\right)$, no observándose esta característica en las medidas realizadas en el sistema $\mathrm{FeMnCu}$. Una razón que puede explicar esta diferencia radica en que desde temperatura ambiente las muestras poseen la fase bcc-FeMnCu que se encuentra magnéticamente ordenada. Esta justificación toma fuerza cuando se observa el efecto de aumentar el campo magnético en el que se realiza el enfriado. Para las muestras estudiadas con un campo de 20 Oe, debido a que el campo coercitivo a temperatura ambiente en cualquiera de las muestras es superior al aplicado en la medida $F C$, es esperable que los valores de la magnetización ZFC y FC no coincidan. Al aumentar el campo magnético de las medidas ZFC-FC a 100 Oe se observa como la irreversibilidad en las curvas aparece a temperaturas inferiores en comparación con la medida realizada a 20 Oe. En las medidas con el campo magnético de 100 Oe se encuentra que la $T_{i}$ disminuye con el contenido de $\mathrm{Cu}$, estando esto relacionado con que el campo coercitivo a temperatura ambiente es más bajo para las muestras con mayor concentración de $\mathrm{Cu}$.

\begin{tabular}{|c|c|c|c|c|c|c|}
\hline & $\mathrm{x}=0.00$ & $\mathrm{x}=0.10$ & $\mathrm{x}=0.15$ & $\mathrm{x}=0.20$ & $\mathrm{x}=0.25$ & $\mathrm{x}=0.30$ \\
\hline$T_{i}\left(\mathrm{H}_{\mathrm{FC}}=20 \mathrm{Oe}\right)$ & $>300 \mathrm{~K}$ & $>300 \mathrm{~K}$ & $>300 \mathrm{~K}$ & $>300 \mathrm{~K}$ & $281 \mathrm{~K}$ & $257 \mathrm{~K}$ \\
\hline$T_{i}\left(\mathrm{H}_{\mathrm{FC}}=100 \mathrm{Oe}\right)$ & $224 \mathrm{~K}$ & $214 \mathrm{~K}$ & $203 \mathrm{~K}$ & $192 \mathrm{~K}$ & $159 \mathrm{~K}$ & $101 \mathrm{~K}$ \\
\hline$T_{Z F C}\left(\mathrm{H}_{\mathrm{FC}}=20 \mathrm{Oe}\right)$ & $246 \mathrm{~K}$ & $201 \mathrm{~K}$ & $185 \mathrm{~K}$ & $171 \mathrm{~K}$ & $134 \mathrm{~K}$ & $116 \mathrm{~K}$ \\
\hline$T_{\mathrm{ZFC}}\left(\mathrm{H}_{\mathrm{FC}}=100 \mathrm{Oe}\right)$ & $241 \mathrm{~K}$ & $196 \mathrm{~K}$ & $179 \mathrm{~K}$ & $163 \mathrm{~K}$ & $127 \mathrm{~K}$ & $110 \mathrm{~K}$ \\
\hline
\end{tabular}

Tabla 5.14: Temperaturas de irreversibilidad $\left(T_{i}\right)$ y del máximo de la curva ZFC $\left(T_{Z F C}\right)$ para las distintas concentraciones y campos magnéticos de enfriado.

La figura 5.77 muestra el efecto del enfriado en presencia de campos magnéticos de 200 Oe, 400 Oe y 4000 Oe en la muestra con mayor contenido de $\mathrm{Cu}$. 


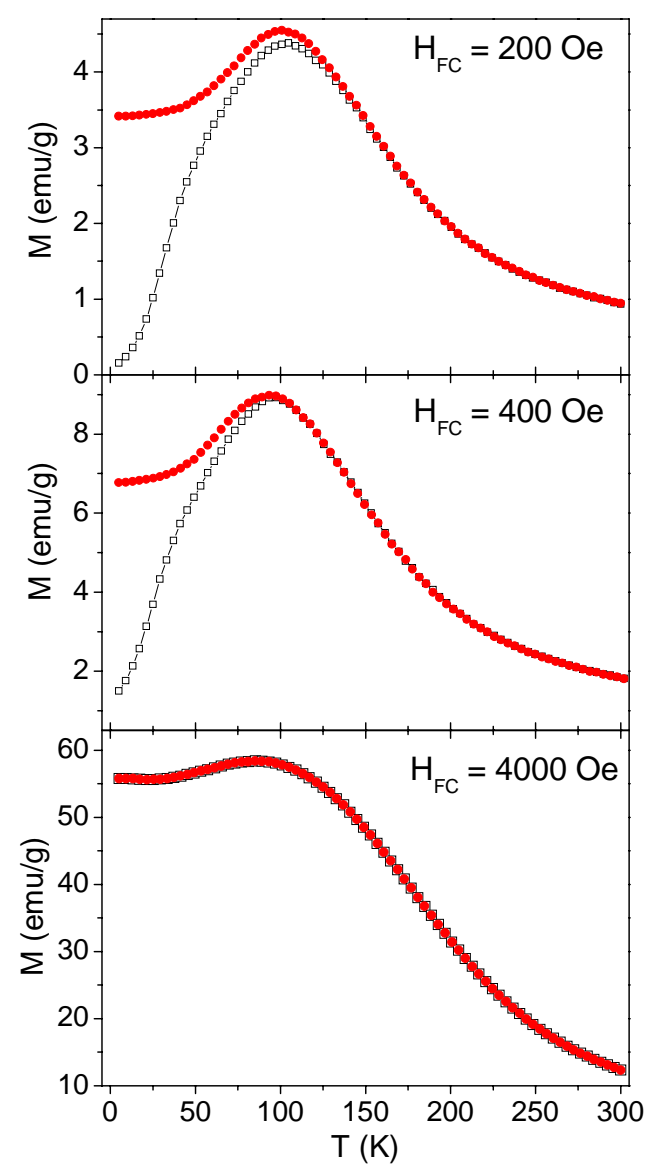

Figura 5.77: Magnetización FC (círculos huecos) y ZFC (cuadrados sólidos) en función de la temperatura en para la muestra $\mathrm{x}=0.30$.

La figura muestra como al continuar incrementando el campo magnético la irreversibilidad de las curvas de magnetización ZFC-FC comienza a temperaturas menores, colapsando a una única curva cuando se aplica un campo de 4000 Oe.

El comportamiento con la temperatura de la curva ZFC es similar al presentado por las curvas de susceptibilidad AC en todas las muestras, observándose una diferencia entre estas dos medidas en la temperatura $T_{Z F C}$ a la cual aparece el cambio de comportamiento en la curva (Tabla 5.14). En las medidas ZFC la temperatura de este punto característico aparece a valores inferiores a las obtenidas en las medidas de susceptibilidad. Esta discrepancia tiene dos orígenes. El primero es que la posición del máximo de la curva ZFC depende del campo magnético en el que se realiza la medida, encontrándose a temperaturas inferiores a medida que la intensidad de dicho campo aumenta. La figura 5.78 muestra tal dependencia para la muestra con $\mathrm{x}=0.30$ en medidas de susceptibilidad AC y de magnetización ZFC en función del campo magnético estático aplicado durante las medidas. Como se mencionó anteriormente, el efecto del campo magnético, en ambas medidas, es desplazar la temperatura del máximo hacia valores más bajos. La línea sólida presente 
en la figura 5.78 es un ajuste de los datos experimentales acorde a lo propuesto por Almeida y Thouless, ${ }^{59}$ según lo cual existirá una relación entre la temperatura del máximo de las curvas de magnetización y el campo magnético aplicado $\left(H_{D C}\right)$ de la forma:

$$
H_{D C} \propto\left(1-\frac{T_{\max }}{T_{C}}\right)^{1 / \gamma}
$$

donde $T_{c}$ es un parámetro a obtener del ajuste que simboliza la temperatura crítica del sistema la cual es el valor al que tiende $T_{\max }$ cuando el campo tiende a cero, y $\gamma$ es el exponente característico de la ley. Para realizar el ajuste de los datos experimentales de la figura 5.8 se reescribió la expresión anterior de la siguiente forma:

$$
T_{\max }=T_{c}-a \cdot\left(H_{D C}\right)^{\gamma}
$$

Los resultados obtenidos empleando la expresión 5.13 son presentados en la Tabla 5.15. El valor obtenido para el exponente característico $\gamma$ está en muy buen acuerdo con el predicho por Almeida y Thouless $^{59}(\gamma=2 / 3)$, y es similar al encontrado en otros sistemas con comportamiento de vidrio de espín. $^{60}$ 


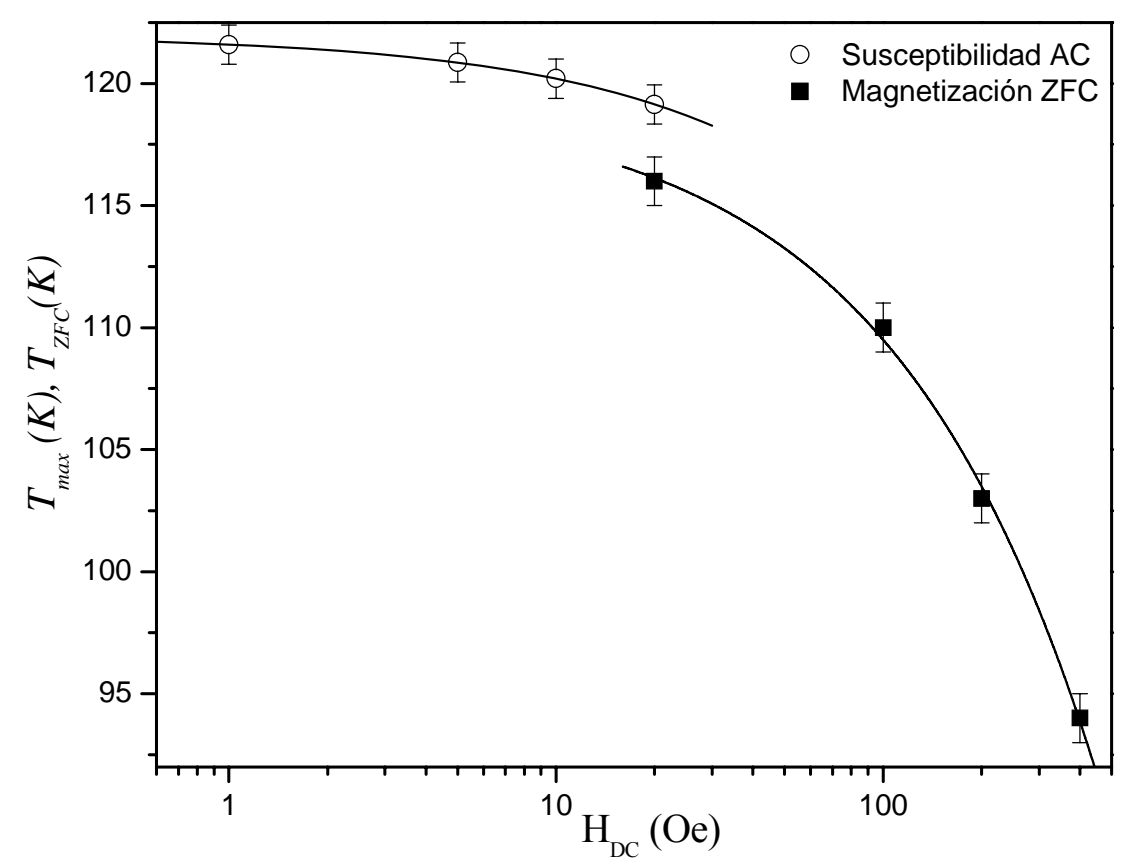

Figura 5.78: Variación de $T_{\max }$ y $T_{Z F C}$ en función del campo magnético. Las líneas sólidas representan el ajuste de los datos experimentales.

\begin{tabular}{|c|c|c|c|}
\hline & $T_{c}$ & $a$ & $\gamma$ \\
\hline Susceptibilidad & $121_{1}$ & $0.28_{1}$ & $0.725_{4}$ \\
\hline Magnetización ZFC & $118_{1}$ & $0.43_{3}$ & $0.68_{1}$ \\
\hline
\end{tabular}

Tabla 5.15: Resultados del ajuste de la temperatura del máximo en función del campo magnético aplicado.

El segundo origen de la diferencia en las temperaturas de los máximos es consecuencia del tiempo característico de medida de cada técnica. En un magnetómetro SQUID este tiempo es de varias decenas de segundos, mientras que en el caso de las medidas de susceptibilidad éste es el inverso de la frecuencia del campo alterno con el cual se realiza la medición, esto es, $10^{-1}$ a $10^{-4}$ segundos. Como se mostró en la sección de resultados de susceptibilidad AC, la temperatura del máximo depende débilmente de la frecuencia del campo de excitación, o dicho de otra forma, la variación es pequeña con el tiempo característico de la técnica a pesar de la gran diferencia entre las ventanas temporales de las mismas. Esta razón explica la diferencia encontrada en la figura 5.79 entre la temperatura del máximo de la susceptibilidad AC $\left(T_{\max }=119 \mathrm{~K}\right)$ y la del máximo de la medida $Z F C\left(T_{Z F C}=116 \mathrm{~K}\right)$ para el campo $H_{D C}=20$ Oe. Con la información aportada por la medida ZFC se puede trazar una gráfica de la temperatura del máximo en función de la ventana temporal de cada experimento. De esta manera se cubre un intervalo que va desde los $10^{-8}$ segundos, tiempo característico de la espectroscopia Mössbauer, hasta la decena de segundos característicos de las medidas realizadas en el magnetómetro. A continuación se muestra en la figura 5.79 el resultado obtenido de graficar el logaritmo de la ventana temporal de cada técnica en función de la inversa de 
la temperatura característica para la muestra con $\mathrm{x}=0.30$. Debido a la imprecisión en la determinación de la temperatura en la medida Mössbauer (cambio de comportamiento en la curva del campo hiperfino medio en función de la temperatura en la figura 5.39) se decidió no incluir este valor en la figura 5.79. Los valores asociados a las medidas de susceptibilidad AC corresponden a la temperatura del máximo en la curva de $\chi^{\prime}$ para distintas frecuencias de medida, mientras que el valor asociado a la medida ZFC corresponde al máximo de la curva medida con un campo magnético continuo de 1 Oe para que dicho campo afecte lo menos posible la posición del máximo. En la figura 5.79 también se presentan el comportamiento esperado según la Ley de Arrhenius (línea punteada) y el ajuste de los datos empleando la Ley de Vogel-Fulcher (línea sólida). Como se ve existe una clara discrepancia entre el comportamiento de los datos experimentales y lo predicho por la Ley de Arrhenius, lo que muestra nuevamente que esta muestra es un sistema de partículas interactuantes.

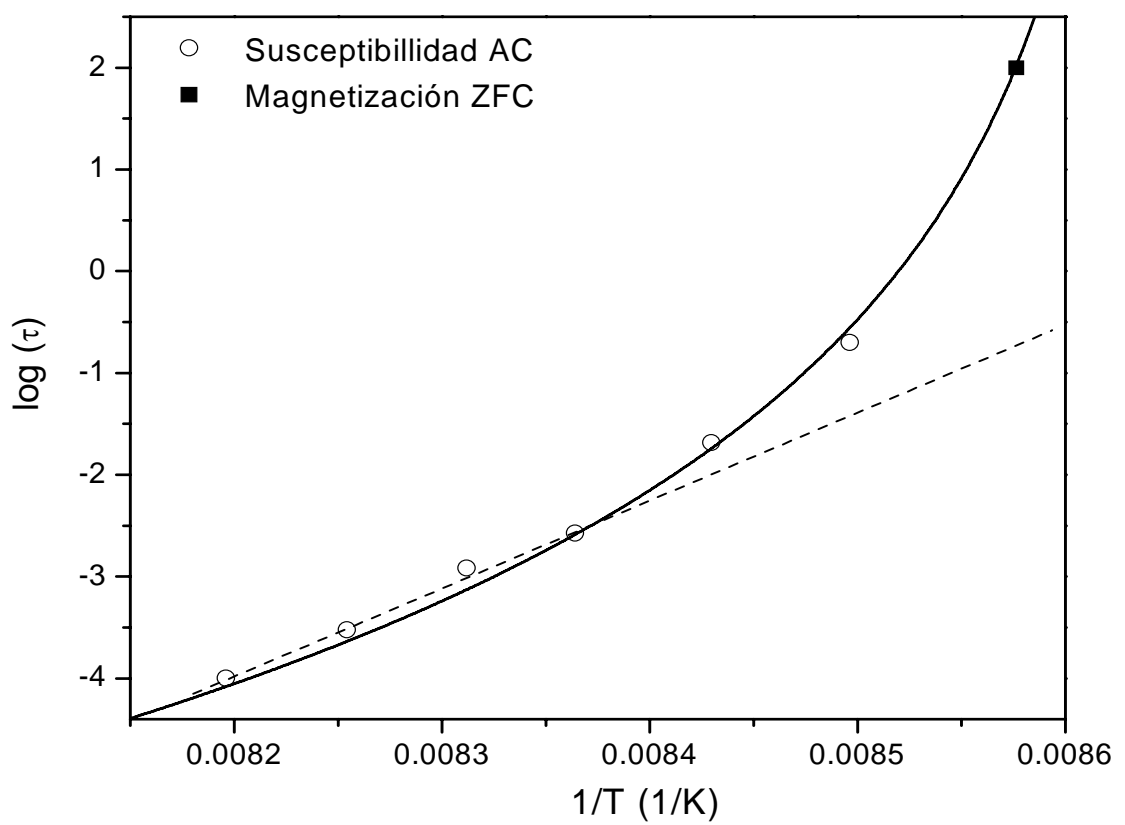

Figura 5.79: Dependencia del logaritmo de la frecuencia de medida con la inversa de la temperatura junto con el comportamiento según la Ley de Arrhenius (línea punteada) y el ajuste empleando la Ley de Vogel-Fulcher (línea sólida).

Algunos autores ${ }^{61}$ han propuesto que la irreversibilidad existente entre las curvas ZFC y FC es producto de la anisotropía magnética que presenten las muestras. Para verificar esta hipótesis proponen la existencia de una relación entre la magnetización de las curvas ZFC y FC dada por:

$$
\frac{\mathrm{M}_{\mathrm{ZFC}}(\mathrm{T})}{\mathrm{H}_{\mathrm{DC}}} \approx \frac{\mathrm{M}_{\mathrm{FC}}(\mathrm{T})}{\mathrm{H}_{\mathrm{DC}}+\mathrm{H}_{\mathrm{c}}^{*}(\mathrm{~T})}
$$


donde $H_{D C}$ es el campo en el que se realizaron las medidas de magnetización y $H_{c}^{*}$ es el campo coercitivo que depende la de temperatura. Aquí, en lugar de hacer esta comparación entre las medidas de magnetización se optó por usar la expresión 5.14 para predecir a partir de las curvas ZFC y FC como sería el comportamiento del campo coercitivo en función de la temperatura, para luego comparar este resultado con el obtenido de los ajustes de los ciclos de histéresis. De la ecuación anterior se obtiene la siguiente forma funcional para $H_{c}^{*}$ :

$$
\mathrm{H}_{\mathrm{c}}^{*} \approx \frac{\left[\mathrm{M}_{\mathrm{FC}}(\mathrm{T})-\mathrm{M}_{\mathrm{ZFC}}(\mathrm{T})\right] \cdot \mathrm{H}_{\mathrm{DC}}}{\mathrm{M}_{\mathrm{ZFC}}(\mathrm{T})}
$$

La figura 5.80 muestra el valor de $H_{c}^{*}$ obtenido empleando los valores de $\mathrm{M}_{\mathrm{FC}}$ y $\mathrm{M}_{\mathrm{ZFC}}$ correspondientes a las medidas realizadas con el campo de 100 Oe, junto con los valores obtenidos para el campo coercitivo $\left(\mathrm{H}_{\mathrm{C} 2}\right)$ obtenido del ajuste de los ciclos de histéresis.
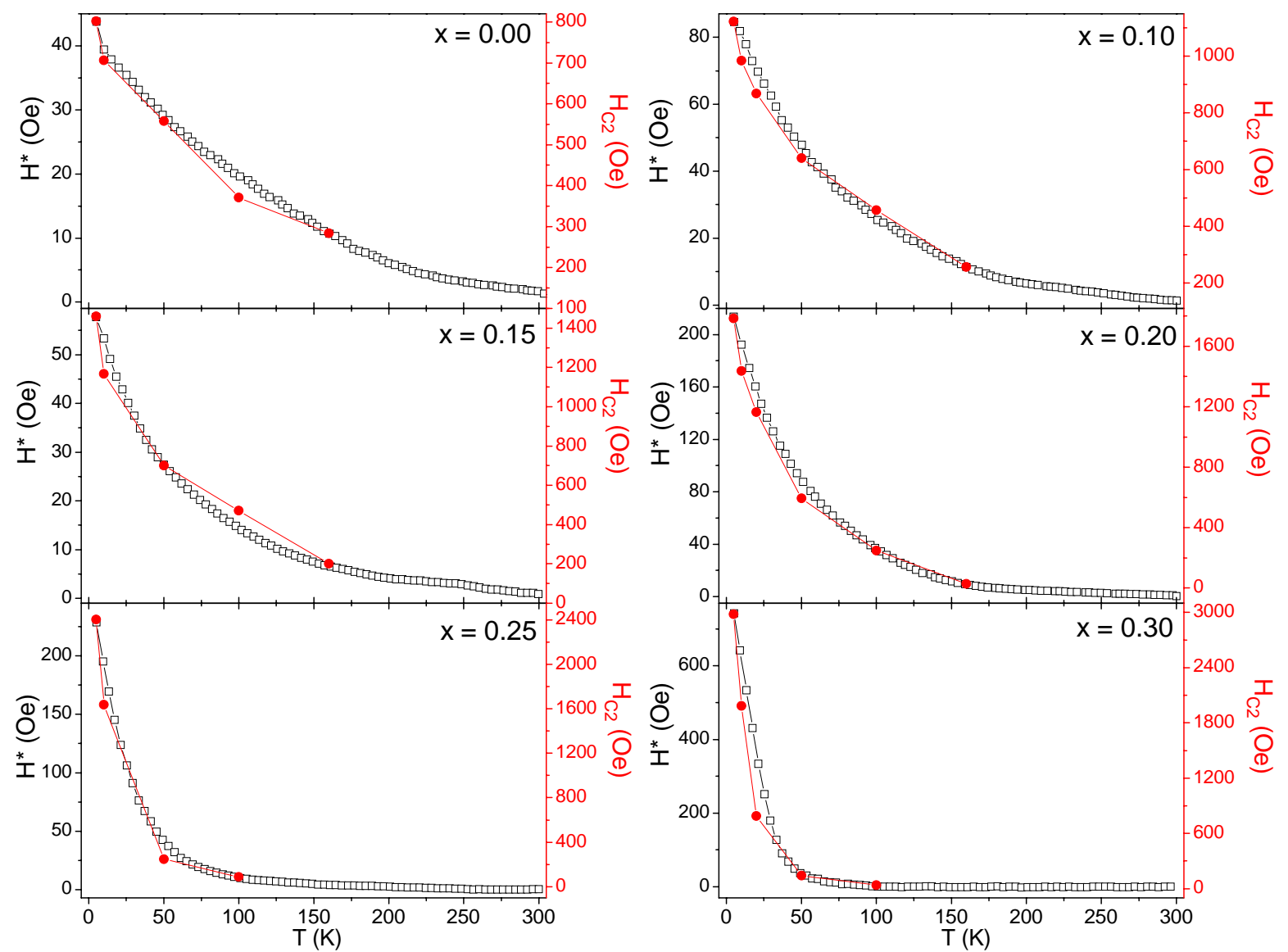

Figura 5.80: Variación del campo coercitivo obtenido con la ecuación 5.14 (cuadrados negros), junto con los resultados provenientes del ajuste de los ciclos de histéresis (círculos rojos). 
Se observa de la figura 5.80 que la forma de las curvas obtenidas empleando la Ec. 5.14 coincide para todas las concentraciones con las obtenidas en el ajuste de los ciclos. Sin embargo, los valores obtenidos difieren significativamente, siendo en todos los casos los valores de $H_{c}^{*}$ inferiores a los de $\mathrm{H}_{\mathrm{C} 2}$. Esta diferencia indica que si bien el campo de anisotropía está influyendo en las curvas ZFC-FC, lo cual se desprende de la forma similar de la figura 5.80, sólo parte de la misma está siendo responsable de la irreversibilidad de las medidas de magnetización. Por otra parte, se debe recordar que una gran diferencia entre estas medidas es que mientras los ciclos de histéresis se miden a una determinada temperatura, las medidas de magnetización se realizan con el sistema fuera del equilibrio termodinámico. En sistemas con características de vidrio de espín esta diferencia puede llevar a que el sistema se comporte de formas diferentes, siendo tanto dependientes de la historia como de las velocidades de calentado o enfriado. 


\section{Resumen de resultados}

A continuación se brinda un resumen de los principales resultados y discusiones presentadas en este Capítulo.

El estudio de la cinética del proceso de aleado de las muestras $\left(\mathrm{Fe}_{79} \mathrm{Mn}_{21}\right)_{85} \mathrm{Cu}_{15}$ y $\left(\mathrm{Fe}_{79} \mathrm{Mn}_{21}\right)_{70} \mathrm{Cu}_{30}$ mostró la evolución de los parámetros estructurales e hiperfinos en función del tiempo del tiempo de molienda. A partir de las $3 \mathrm{~h}$ de molienda la fase mayoritaria para todas las muestras es la de estructura $f c c$ con un parámetro de red que aumenta con el tiempo de molienda hasta llegar a su valor estacionario de $3.614 \AA$ y $3.625 \AA$ para las muestras con $\mathrm{x}=0.15 \mathrm{y} \mathrm{x}=0.30$, respectivamente. Este aumento es atribuido tanto al proceso de molienda en sí mismo como a la formación de la solución sólida $\mathrm{FeMnCu}$. El tamaño de cristalita disminuye con el tiempo de molienda hasta alcanzar un valor estacionario de $9 \mathrm{~nm}$ para ambas muestras. La EM indica la presencia de dos interacciones, una de alto campo y otra de bajo campo. La primera de ellas está relacionada con la fase $b c c$ presente en los difractogramas, y se encuentra en ambas muestras para 1 $\mathrm{h}$ de molienda donde es la fase mayoritaria. Para tiempos posteriores dicha interacción sólo puede ser observada en la muestra con $\mathrm{x}=0.15$. La distribución de campos magnéticos hiperfinos asociada a la fase $b c c$ indica que el Fe tiene distintos entornos con cero, uno y dos o más átomos primeros vecinos de $\mathrm{Mn}$ o $\mathrm{Cu}$. Por su parte la segunda interacción hiperfina magnética está relacionada con la fase $f c c$. Los parámetros de dicha interacción aumentan con el tiempo de molienda, alcanzando su estado estacionario a partir de las $12 \mathrm{~h}$. La evolución tanto de los parámetros estructurales como los hiperfinos obtenidos por DRX y EM, respectivamente, muestra que para un tiempo de molienda de $12 \mathrm{~h}$ el sistema alcanza un estado estacionario, por lo que un tiempo de $15 \mathrm{~h}$ resulta ser indicado para arribar a dicho estado, sin aumentar significativamente la contaminación con el material de los cilindros y las esferas.

Los parámetros estructurales con el contenido de $\mathrm{Cu}$ muestran un aumento del parámetro de red de la estructura $f C C$ con una tasa de incremento promedio de $7.8_{2} \times 10^{-4} \AA / \%$ at de $\mathrm{Cu}$. Este aumento está asociado con la incorporación substitucional de átomos de $\mathrm{Cu}$ en la estructura fcc.

El tamaño medio de cristalita determinado usando la formula de Scherrer, resulta de $9 \mathrm{~nm}$ para todas las muestras independientemente del contenido de $\mathrm{Cu}$, indicando que no se favorecen procesos de soldadura ni de fractura con el agregado de $\mathrm{Cu}$.

De la región XANES de los espectros de absorción de rayos X se concluye que ni el Fe ni el $\mathrm{Cu}$ se encuentran oxidados. La región EXAFS muestra, en acuerdo a lo observado por DRX, un aumento en la distancia a primeros vecinos de $5.6_{3} \times 10^{-4} \AA \AA / \%$ at. de $\mathrm{Cu}$. El número de átomos de $\mathrm{Cu}$ en la primera esfera de coordinación también presenta un cambio lineal con el contenido de $\mathrm{Cu}$, 
siendo esto otra evidencia de que este último se está incorporando substitucionalmente en la estructura $f c c$. La comparación de este resultado con el correspondiente al número más probable de átomos de $\mathrm{Cu}$ primeros vecinos obtenido a partir de un modelo de distribución al azar de átomos en la estructura $f c c$, sugiere la formación de una solución sólida substitucional y aleatoria.

Los parámetros hiperfinos de la fase bcc obtenidos a temperatura ambiente muestran que el campo magnético hiperfino medio y el corrimiento isomérico medio de la distribución de alto campo son independientes del contenido de $\mathrm{Cu}$, estabilizándose en valores de $31 \mathrm{~T}$ y $-0.01 \mathrm{~mm} / \mathrm{s}$, respectivamente. El valor del campo hiperfino permite asegurar que los átomos de Mn están formando parte de la estructura del bcc-Fe. En lo que respecta a la presencia de átomos de $\mathrm{Cu}$ en esta estructura, debido al menor cambio que los mismos producen en el campo hiperfino del Fe y la baja proporción de esta interacción, no es posible asegurar si el $\mathrm{Cu}$ se encuentra presente en la fase bcc.

Por otra parte, el campo hiperfino medio asociado a la fase $f c c$ determinado a temperatura ambiente disminuye al aumentar la concentración de $\mathrm{Cu}$ debido a la sustitución de un átomo magnético $(\mathrm{Fe}$ o $\mathrm{Mn})$ por uno no magnético $(\mathrm{Cu})$. El corrimiento isomérico medio muestra un aumento con la presencia del $\mathrm{Cu}$ dado por la expresión $\delta(\mathrm{x})=0.25_{2} \mathrm{x}-0.05_{1}(\mathrm{~mm} / \mathrm{s})$. Esta variación está relacionada con el aumento en la densidad de electrones $3 d$ alrededor del núcleo de Fe, sumado a una leve contribución proveniente del aumento del volumen de la celda originada por el $\mathrm{Cu}$. Las distribuciones de probabilidad del campo magnético hiperfino presentan una contribución a campo cero asociada con la presencia de fallas de apilamiento en la estructura $f_{c c}$. Los resultados de las medidas realizadas a bajas temperaturas revelan la existencia de una temperatura característica en la cual el campo hiperfino promedio de la fase $f c c$ aumenta, indicando que un proceso de orden magnético tiene lugar. Esta temperatura es dependiente de la concentración de $\mathrm{Cu}$, siendo más baja para las concentraciones más altas. Los valores que alcanza el campo hiperfino a bajas temperaturas también aumentan con el contenido de $\mathrm{Cu}$, llegando a valores de hasta $10 \mathrm{~T}$ para la muestra con $\mathrm{x}=0.30$. El corrimiento isomérico medio aumenta con el contenido de $\mathrm{Cu}$, siendo las muestras con mayor contenido de $\mathrm{Cu}$ las que presentan un corrimiento isomérico más alto, manteniéndose este comportamiento en todo el rango de temperaturas. La evolución del corrimiento isomérico con la temperatura puede ser descripta teniendo en cuenta la contracción de la red cristalina con el descenso de temperatura y los efectos contemplados en el corrimiento Doppler de segundo orden.

Las medidas de la susceptibilidad magnética AC presentan dos comportamientos diferentes para las muestras con bajos y altos contenidos de $\mathrm{Cu}$. Para $\mathrm{x} \leq 0.15$ la susceptibilidad es creciente en la región de bajas temperaturas y luego tiende a permanecer constante. Este comportamiento está ligado con la fase bcc ordenada ferromagnéticamente que se observa en DRX y en EM. Para $\mathrm{x} \geq$ 0.20 las curvas de susceptibilidad muestran un claro máximo. Tanto la temperatura del cambio de 
comportamiento en las curvas para bajos contenidos de $\mathrm{Cu}$, como la temperatura correspondiente al máximo de la susceptibilidad para las más altas concentraciones muestran una evolución lineal con la concentración de $\mathrm{Cu}$, encontrándose en buena coincidencia con las temperaturas donde el campo hiperfino de la fase $f c c$ comienza a crecer. Para las muestras con altos contenidos de $\mathrm{Cu}$, esto es $\mathrm{x} \geq$ 0.20 , el comportamiento del máximo de la susceptibilidad es poco dependiente de la frecuencia del campo de medida, con valores de la sensitividad por década de frecuencia del orden de los encontrados en vidrios de espín. La necesidad de emplear la ley de Vogel-Fulcher en el comportamiento de $T_{\max }$ indica que existen interacciones entre partículas. El análisis basado en la ley de exponentes críticos muestra valores de los parámetros $z v$ y $\tau_{0}$ que junto con, la disminución de $T_{\max }$ ante la presencia de un campo magnético DC y la respuesta del término no-lineal de la susceptibilidad, indican que el sistema posee las características típicas de un vidrio de espín. Los ciclos de histéresis de las muestras con diferentes concentraciones de $\mathrm{Cu}$ y en función de la temperatura, fueron reproducidos en la mayoría de los casos empleando tres contribuciones, dos ferromagnéticas y una lineal. La primera de las contribuciones ferromagnéticas es asociada a la fase $b c c$, observada también por DRX y EM. La magnetización de saturación de esta contribución es mayor para las muestras con menos $\mathrm{Cu}$ y la fracción obtenida a partir de la comparación con una muestra de $\mathrm{Fe}_{90} \mathrm{Mn}_{10}$, está en buen acuerdo con la fracción de la interacción de alto campo encontrada en EM. La variación con el contenido de $\mathrm{Cu}$ de la magnetización y del campo coercitivo de esta contribución permiten concluir que existen átomos de $\mathrm{Cu}$ formando parte de la estructura $b c c$, cuestión que no había podido ser determinada por los resultados de EM. La segunda de las contribuciones ferromagnéticas tiene un claro correlato con el orden que aparece a bajas temperaturas. Los valores de la magnetización de saturación y el campo coercitivo asociados a ésta aumentan significativamente por debajo de $T_{\max }$, indicando nuevamente que dos regímenes magnéticos ocurren por encima y por debajo de esta temperatura. Los ciclos medidos en presencia de campo magnético durante el enfriado exhiben el fenómeno de exhange bias, encontrándose que el campo $H_{E B}$ depende fuertemente del campo aplicado durante el enfriado. Estos indicios permiten hacer la hipótesis que las partículas del sistema $\mathrm{FeMnCu}$ están conformadas por un núcleo ordenado magnéticamente y una región superficial que es la responsable del comportamiento tipo vidrio de espín. El valor del exponente obtenido de ley de Almeida y Thouless refuerza la hipótesis de la presencia de un arreglo core-shell. 


\subsection{Referencias}

\footnotetext{
${ }^{1}$ JCPDS- Internacional Center for Diffraction Data, PCPDFWIN v.2.1, tarjeta No 06-0696.

${ }^{2}$ P. Scherrer; Nachr. Ges. Wiss. Gottingen 26, (1918), pág 98.

${ }^{3}$ JCPDS- Internacional Center for Diffraction Data, PCPDFWIN v.2.1, tarjeta $\mathrm{N}^{\mathrm{o}} 32-0637$.

${ }^{4}$ JCPDS- Internacional Center for Diffraction Data, PCPDFWIN v.2.1, tarjeta $\mathrm{N}^{\mathrm{o}} 85-1326$.

${ }^{5}$ C. Kittel, in "Introduction to Solid State Physics", 6 ${ }^{\text {ta }}$ edición, J. Wiley \& Sons, New York, (1986), pág. 405.
}

6 D.R. Lide (editor), en "CRC Handbook of Chemistry and Physics", Version 2009, http://www.hbcpnetbase.com, CRC Press, Boca Raton, FL, (2009), pág 12-119.

${ }^{7}$ ElementData, programa "Mathemática” versión 6.0, Wolfram Research, (2008).

${ }^{8}$ M. Mizrahi, S.J Stewart, A.F. Cabrera y J. Desimoni; Hyp. Int. 161 (2005), pág. 171.

${ }^{9}$ R.S. Preston, S.S. Hanna y J. Heberle; Phys. Rev. 128, (1962), pág. 2207.

${ }^{10}$ C. Paduani, E. Galvão da Silva y G.A. Perez-Alcazar; J. Appl. Phys. 70 ,12 (1991), pág. 7524.

${ }^{11}$ J. Martinez, S.M. Cotes y J. Desimoni; Journal of Alloys and Comp. 479, (2009), pág. 204.

${ }^{12}$ A. Forsberg y J. Agren; Journal of phase equilibria 14, 3, (1993), pág. 354.

${ }^{13}$ R. Delhez, T.H. Keijser, J.I. Langford, D. Louer, E.J. Mittemeijer y E.J. Sonnevel, en “The Rietveld Rietveld Method”, R.A. Young (editor), IUCr, Monograph No5, Oxford University Press, Ch. 8, (1993).

14 J. Martínez, S.M. Cotes, A.F. Cabrera, J. Desimoni y A. Fernández Guillermet; Mat. Sci. \& Eng. A 408, 1-2 (2005), pág. 26.

${ }^{15}$ V.V. Tcherdyntsev, S.D. Kaloshkin, I.A. Tomolin, E.V. Shelekhov y Y.V. Baldokhin; Z. Metallkde 90, 9 (1999), pág. 747.

${ }^{16}$ V.V.Tcherdyntsev, S.D. Kaloshkin, I.A. Tomolin y E.V. Shelekhov; Nanostruc. Mater. 12, 1-4 (1999), pág. 139.

17 J.Z. Jiang, C. Gente y R. Bormann; Mater. Sci. Eng. A 242, 1-2 (1998), pág. 268.

18 D.R. Lide (editor), en "CRC Handbook of Chemistry and Physics", Version 2009, http://www.hbcpnetbase.com, CRC Press, Boca Raton, FL, (2009).

19 M. Wilke, F. Farges, P.E. Petit, G.E. Brown Jr. y F. Martin; American Mineralogist 86, 5-6 (2001), pág. 714 .

${ }^{20}$ C. Lamberti, S. Bordiga, F. Bonino, C. Prestipino, G. Berlier, L. Capello, F. D'Acapito, F. X. Llabrés i Xamena y A. Zecchina; Phys. Chem. Chem. Phys. 5, (2003), pág. 4502. 
${ }^{21}$ G. Azevedo, presentación en "School on Nanostructured Materials: Characterization by Synchrotron Light based Techniques", Instituto de Investigaciones Fisicoquímicas Teóricas y Aplicadas (INIFTA), 18-23 de noviembre, (2007).

${ }^{22}$ P. Bandyopadhyay y C.U. Segre; http://www.csrri.iit.edu/mucal.html.

${ }^{23}$ M.B. Stearns; Phys. Rev. 147, 2 (1966), pág. 439.

${ }^{24}$ C.L. Chien, S.H.Liou, D. Kofalt, W. Yu, T. Egami y T.R. McGuire; Phys. Rev. B 33, 5 (1986), pág. 3247.

${ }^{25}$ M. Mizrahi; Trabajo de Diploma de la Licenciatura en Física de la Facultad de Ciencias Exactas, Universidad Nacional de La Plata, (2005).

${ }^{26}$ M. Mizrahi, A.F. Cabrera, S.M. Cotes, S.J. Stewart, R.C. Mercader y J. Desimoni; Hyp. Int. 156-157, 1-4 (2004), pág. 541.

${ }^{27}$ B.Balko y G.R. Hoy; Phys. Rev. B 10, (1974), pág. 4523.

${ }^{28}$ C. Paduani, E. Galvao da Silva y G.A. Pérez Alcázar; Hyp Int. 73, 3-4 (1992), pág. 233.

${ }^{29}$ J. Martinez, S.M. Cotes y J. Desimoni; Phys. Stat Solidii b, 246, (2009), pág. 1366.

${ }^{30}$ V.V. Tcherdyntsev, L.Y. Pustov, S.D. Kaloshkin, E.V. Shelekhov y G. Principi; Hyp. Int. 168, 1-3 (2006), pág. 937.

${ }^{31}$ M. Uhrmacher, A. Kulinska, Yu.V. Baldokhin. V.V. Tcherdyntsev, S.D. Kaloshkin, A. Maddalena y G. Principi; Intermetallics 10, (2002), pág. 571.

${ }^{32}$ A. Dumay, J.P. Chateau, S. Allain, S. Migot y O. Bouaziz; Mat. Sci. and Eng. A 483-484, (2008), pág. 184.

${ }^{33}$ B.E. Warren, en “X-Ray Diffraction”, Addison-Wesley, (1969), pág. 289.

34 M. K. Roy y H. C. Verma; J. Magn. Magn. Mat. 270, (2004), pág. 186.

${ }^{35}$ C. W. Kimball; Phys. Rev. 146, (1966), pág. 375.

${ }^{36}$ D.L. Williamson, en "Mössbauer Isomer Shifts", G.K. Shenoy and F. E. Wagner (editores), North Holland, Amsterdam, New York, Oxford, (1978), pág. 337.

${ }^{37}$ L. M. Socolovsky y F.H. Sánchez; J. Met. and Noncryst. Mat. 22, (2004), pág. 97.

${ }^{38}$ J. Restrepo y G.A. Pérez Alcázar; J. Appl. Phys. 87, 10 (2000), pág. 7425.

${ }^{39}$ A. Aharoni, en "Introduction of the theory of ferromagnetism", Clarendon press, Oxford, (1996), pág. 86.

${ }^{40}$ J.A. Mydosh, en "Spin glasses: an experimental introduction", Taylor \& Francis, London-Washinton, DC (1993), pág. 67.

41 J. A. Mydosh, en "Spin glasses: an experimental introduction", Taylor \& Francis, London-Washinton, DC (1993), pág. 66.

${ }^{42}$ S. Shtrikman y E.P. Wohlfarth; Phys. Letters A, 85, 8-9 (1981), pág. 467.

${ }^{43}$ C. Djurberg, P. Svedlindh, P. Nordblad, M.F. Hansen, F. Bødker y S. Mørup; Phys. Rev. Lett. 79, 25 (1997), pág. 5154. 
${ }^{44}$ P. Jönsson, M.F. Hansen, P. Svedlindh y P. Nordblad; J. Magn. Magn. Mater. 226-230, part 2 (2001), pág. 1315.

45 J. A. Mydosh, en "Spin glasses: an experimental introduction", Taylor \& Francis, London-Washinton, DC (1993), pág. 110.

${ }^{46}$ J.A. De Toro, M.A. López de la Torre y J.M Riveiro; Phys. Rev. 60, 18, (1999), pág. 12918.

${ }^{47}$ O. Cador, F. Grasset, H. Hanedab y J. Etourneaud; J. Magn. Magn. Mater. 268, (2004), pág. 232.

${ }^{48}$ M.F. Hansen, C.B. Koch y S. Mørup; Phys. Rev. B 62, (2000), pág. 1124

${ }^{49}$ D. Jiles, en "Introduction to magnetism and magnetic materials", 2 ${ }^{\text {da }}$ edición, CRC Press, (1998), pág. 105.

50 A. Aharoni, en "Introduction to the Theory of Ferromagnetism", $1^{\text {ra }}$ edición, Clarandon Press, Oxford, (1998), pág. 14.

${ }^{51}$ S.A. Makhlouf y F.T. Parker; Phys. Rev. B 55, 22 (1997), pág. R14 717.

${ }^{52}$ J. Nogués, J. Sort, V. Langlais, V. Skumryev, S. Suriñach, J.S. Muñoz y M.D. Baró; Phys. Rep. 422 , (2005), pág. 65.

${ }^{53}$ L. Del Bianco, D. Fiorani, A.M. Testa, E. Bonetti y L. Signorini; Phys. Rev. B 70, (2004), pág. 052401-

${ }^{54}$ W.H. Meiklejohn y C.P. Bean; Phys. Rev. 105, 3 (1956), pág. 904.

${ }^{55}$ P.J. van der Zaag, R.M. Wolf, A.R. Ball, C. Bordel, L.F. Feiner y R. Jungblut; J. Magn. Magn. Mater. 148, (1995), pág. 346

${ }^{56}$ R.H. Kodama, A.E. Berkowitz, E.J. McNiff y S. Foner; Phys. Rev. Lett. 77, (1996), pág. 394.

${ }^{57}$ R.H. Kodama, S.A. Makhlouf y A.E. Berkowitz; Phys. Rev. Lett. 79, (1997), pág.1393.

58 J.A. De Toro, M.A. López de la Torre, M. A. Arranz, J.M. Riveiro y J.L. Martínez; Phys. Rev. B, 64, (2001), pág. 094438.

59 J.R.L. de Almeida y D.J. Thouless; J. Phys. A 11, (1978), pág. 983.

${ }^{60}$ H. Wang, T. Zhu, K. Zhao,W.N. Wang, C.S. Wang, Y.J. Wang y W.S. Zhan; Phys. Rev. B 70, (2004), pág. 092409-1.

${ }^{61}$ P.A. Joy, P.S. Anil Kumar y S.K. Date; J. Phys.: Condens. Matter 10, (1998), pág. 11049. 


\section{Capítulo 6. Conclusiones}

Las propiedades estructurales, hiperfinas y magnéticas del sistema $\left(\mathrm{Fe}_{79} \mathrm{Mn}_{21}\right)_{1-\mathrm{x}} \mathrm{Cu}_{\mathrm{x}}(0.00 \leq$ $\mathrm{x} \leq 0.30)$ preparado por molienda mecánica han sido estudiadas en forma sistemática mediante Difracción y Absorción de rayos X, Espectroscopía Mössbauer en función de la temperatura, y medidas de magnetización vs. campo y temperatura y susceptibilidad AC.

Este nuevo y sistemático conjunto de resultados permite concluir que:

- El proceso de mezclado es progresivo hasta las $9 \mathrm{~h}$ de molienda, para tiempos mayores el sistema llega a un estado estacionario en el cual el tamaño de cristalita, los parámetros de red e hiperfinos no presentan cambios en los tiempos sucesivos. Por ese motivo $15 \mathrm{~h}$ de molienda es un tiempo razonable para la estabilización del sistema no observándose contaminación apreciable con el material de los cilindros y bolas usados en el proceso. La evolución de estos parámetros con el tiempo de molienda sugiere la presencia de inhomogeneidades composicionales en las primeras etapas y la formación de una solución sólida homogénea en las últimas.

- El comportamiento del tamaño de cristalita con el contenido de $\mathrm{Cu}$ indica que éste no modifica el balance entre los procesos de soldadura y fractura.

- La evolución de la contribución a campo nulo de la distribución de probabilidad del campo hiperfino asociada a la fase $f c c$ junto con la variación de la energía de formación de fallas de apilamiento con el contenido de $\mathrm{Cu}$, permiten asociar estas contribuciones a dichas fallas, aunque la presencia de una fase superparamagnética a temperatura ambiente no pueda ser completamente descartada. 
- La variación de los parámetros estructurales e hiperfinos a temperatura ambiente con el contenido de $\mathrm{Cu}$ junto con cálculos de probabilidades de distribución de átomos en una red fcc permiten concluir que bajo molienda se forma una solución sólida $\mathrm{Fe}-\mathrm{Mn}-\mathrm{Cu}$ substitucional y aleatoria.

- El máximo observado en la susceptibilidad AC junto con la débil variación de su posición con la frecuencia del campo aplicado, los valores de exponente crítico obtenidos, el cambio con la temperatura del coeficiente del término no-lineal de la susceptibilidad, el comportamiento de la magnetización en enfriado con campo y la evolución del campo hiperfino con la temperatura sugieren que el sistema se comporta, en el rango de composiciones estudiado, como un conjunto de partículas interactuantes con las características de un vidrio de espín .

- La presencia de exchange bias y la fuerte dependencia de éste con el campo aplicado durante el enfriado, permiten modelar a las partículas como un núcleo ordenado magnéticamente y una superficie desordenada que es la responsable del comportamiento tipo vidrio de espín.

\section{Perspectivas}

Para continuar el estudio del sistema $\mathrm{FeMnCu}$, se proponen realizar algunas de las tareas siguientes, las cuales contribuirán a ampliar los conocimientos sobre el mismo.

> Realizar tratamientos térmicos en las muestras y su posterior análisis con las técnicas presentadas en esta Tesis, para estudiar como son afectadas las propiedades de las mismas por los posibles cambios estructurales y magnéticos producto del tratamiento.

> Realizar un estudio de las propiedades estructurales a bajas temperaturas.

$>$ Realizar estudios de magnetorresistencia, ya que el sistema es un buen candidato a presentar estas características.

$>$ Realizar medidas del tiempo de relajación magnética del sistema.

> Realizar simulaciones Montecarlo para intentar reproducir la respuesta magnética del sistema. 


\section{Producción Científica}

Trabajos publicados a partir de resultados presentados en esta Tesis:

1. "Structural and magnetic study of nanostructured (Fe79Mn21)80Cu20 alloy synthesized by ball milling"

M. Mizrahi, F.A. Cabrera, S.J. Stewart, H.E. Troiani, S.M. Cotes y J. Desimoni.

Physica B - condensed matter, 354, 1-4 (2004), pág. 133.

2. "Mössbauer investigation of femncu nanostructured alloys obtained by ball milling” M. Mizrahi, S.J. Stewart, A.F. Cabrera y J. Desimoni.

Hyperfine Interactions 161, 1-4 (2005), pág. 171.

3. "Study of the evolution of the hyperfine parameters in nanostructured Fe-Mn-Cu system"

M. Mizrahi, A.F. Cabrera y J. Desimoni.

Hyperfine Interactions 179, 1-3 (2007), pág. 39.

4. "Structural and hyperfine evolution of the $\left(\mathrm{Fe}_{79} \mathrm{Mn}_{21}\right)_{1-\mathrm{x}} \mathrm{Cu}_{\mathrm{x}}$ system under milling time"

M. Mizrahi, A.F. Cabrera y J. Desimoni.

Journal of Alloys and Compounds, (2009) (en prensa).

Versión electrónica: http://dx.doi.org/10.1016/j.jallcom.2009.10.166.

5. "Spin glass behavior of mechanically alloyed $f c c-\left(\mathrm{Fe}_{79} \mathrm{Mn}_{21}\right)_{1-\mathrm{x}} \mathrm{Cu} \mathrm{u}_{\mathrm{x}}(0.00 \leq \mathrm{x} \leq 0.30)$ " M. Mizrahi, A.F. Cabrera y J. Desimoni.

Journal of Applied Physics 107, 4 (2010), pág. 044311.

Otras publicaciones relacionadas con el trabajo de esta Tesis:

6. "Distribution of Mn atoms in a substitutional bcc-Fe(Mn) solid solution"

M. Mizrahi, A.F. Cabrera, S. Cotes, S.J. Stewart, R.C. Mercader y J. Desimoni.

Hyperfine interactions 156, 1-4 (2004), pág. 541.

Otras publicaciones realizadas durante el período del Doctorado:

7. “Nonmonotonic reversible branch in four model granular beds subjected to vertical vibration"

L. Pugnaloni, M. Mizrahi, C. Carlevaro y F. Vericat.

Physical review E 78, 5 (2008), pág. 051305. 


\section{Agradecimientos}

Todos los Capítulos de esta Tesis han llevado un tiempo significativo de elaboración y como no puede ser de otra forma la sección de los agradecimientos no ha sido la excepción. Será porque uno quiere plasmar en unas pocas líneas el infinito agradecimiento que se tiene hacia con la gente que a lo largo de todo este proceso ha colaborado de una $\mathrm{u}$ otra manera para hacer mi tarea más fácil.

En primer lugar quiero agradecer a mi directora Judith Desimoni y mi co-directora Fabiana Cabrera. Ellas han sabido guiarme por la senda del conocimiento científico, aportando todo su saber y experiencia, trabajando en un ámbito tanto profesional como cordial, cualidades indispensables para alcanzar los objetivos planteados.

Quiero agradecer a los miembros del jurado por haber aceptado dicho labor, en especial al Dr. Francisco Sánchez quien a pesar de tener que evaluar otra Tesis en el mismo mes se hizo el tiempo para poder ser parte de este tribunal.

Quiere brindarle un agradecimiento muy especial a Silvana Stewart quien desde el comienzo de mi trabajo me ayudó con sus conocimientos de magnetismo, compañía durante turnos de medidas y su apoyo, incondicional y desinteresado, el cual estuvo presente hasta los últimos momentos de esta Tesis.

A todos mis compañeros de grupo de trabajo, en especial al patriarca del grupo Roberto Mercader, por enseñarme todo lo que sabe, que no es poco. A la parte técnica, Cacho Ghisolfi, Jorge Runco, Luis Junciel, siempre dispuestos a sacarme de problemas cuando los equipos se ponían rebeldes, y en especial a Flavio Sives por ayudarme con las 
medidas de susceptibilidad e posterior interpretación de los resultados. A mis compañeros Tesistas, Javier Martinez, Luciana Montes y Arlés Gil Rebaza.

A todos los miembros del LENIH, con los cuales uno comparte muchas horas diariamente y ya son como una segunda familia para mí, con sus defectos y virtudes, como sucede en todas las familias que se precien.

Dos personas, que a pesar de no tener con ellos un contacto cotidiano, siempre que nos cruzamos se interesan por saber como andan mis cosas. A Luís Epele y Osvaldo Civitarese mi gratitud por ello.

A los muchachos del paddle (Quique, Toto, Vasco, Carlos), por compartir buenos momentos descargando las broncas golpeando la pelota.

A mis mejores amigos German Darriba, Lisandro Giovanetti y Martín Falabella por su ayuda desde lo profesional hasta su amistad incondicional, saber que siempre se puede contar con ellos es invalorable. Un agradecimiento especial a Vitaly Bilovol con quien compartimos un largo y caluroso enero en el Departamento de Física, su compañía ha hecho que la escritura de la Tesis se haga más llevadera.

A Pía, quien me ha ayudado de tantas formas que no me alcanzaría otra Tesis para mencionarlas, gracias por estar siempre y bancarme, sobretodo en este último tramo en el que mi locura habitual estaba potenciada. A ella mi eterno amor y agradecimiento.

Por supuesto a mi familia (Cristina, Daniel y Federico), siempre apoyándome cuando las cosas iban bien y sobre todo cuando el rumbo parecía incierto. Finalmente a mi perro Tomás, por su compañía echado a mis pies durante esas largas noches de escritura y por sus constantes demandas para salir a dar un paseo, lo cual sin saberlo él, me estaba beneficiando más a mí. 